(C)2009

JAY L. ADAMS

ALL RIGHTS RESERVED 


\title{
HANKEL OPERATORS FOR FRACTIONAL-ORDER SYSTEMS
}

\author{
A Dissertation \\ Presented to \\ The Graduate Faculty of The University of Akron
}

\author{
In Partial Fulfillment \\ of the Requirements for the Degree \\ Doctor of Philosophy
}

Jay L. Adams

August, 2009 


\title{
HANKEL OPERATORS FOR FRACTIONAL-ORDER SYSTEMS
}

\author{
Jay L. Adams
}

\author{
Dissertation
}

Approved:

Accepted:

Advisor

Department Chair

Dr. Tom T. Hartley

Dr. J. Alexis De Abreu Garcia

Co-Advisor

Dean of the College

Dr. Robert J. Veillette

Dr. George K. Haritos

Committee Member

Dean of the Graduate School

Dr. J. Alexis De Abreu Garcia

Dr. George R. Newkome

Committee Member

Dr. D. Dane Quinn

Date

Committee Member

Dr. Gerald W. Young 


\begin{abstract}
This dissertation presents an algorithm utilizing four unitarily equivalent representations of the Hankel operator to estimate the Hankel singular values of fractional-order systems based on the Rayleigh-Ritz method. This algorithm is applied to estimate the first ten Hankel singular values for systems of the forms $\frac{a}{s^{\frac{1}{2}}+a}$ for $a \in \mathbb{R}_{+}$and $\frac{1}{s^{q}+1}$ for $q \in(0,2)$. Error bounds are generated for each Hankel singular value estimate. The algorithm is also applied to two physical fractional-order systems, the resistor-terminated and inductor-terminated semi-infinite lines, to show how it can be used. Hankel norms are also approximated for conjugate-order systems of the form $\frac{\frac{1}{2}}{s^{q}+1}+\frac{\frac{1}{2}}{s^{\bar{q}}+1}$ for a variety of $q \in \mathbb{C}$ to highlight the flexibility of the algorithm in Hankel-norm computation for a wide variety of systems.
\end{abstract}




\section{ACKNOWLEDGEMENTS}

I would like to thank Dr. Tom T. Hartley for his support, example, and enthusiasm. His understanding of fractional-order systems has helped shape my understanding greatly. I would also like to thank Dr. Robert J. Veillette for pushing me toward excellence and for his expertise in $H_{\infty}$ control. I would like to thank Dr. J. Alexis de Abreu-Garcia for his confidence in me, both in research and teaching. Also, I would also like to thank Dr. D. Dane Quinn and Dr. Gerald W. Young for helping me discover ways to explain things more clearly. I would like to thank Dr. J. Patrick Wilber for his influence in my understanding of functional analysis. The whole faculty in the Department of Electrical and Computer Engineering and in the Department of Theoretical and Applied Mathematics has been very helpful in my time at the University of Akron.

I appreciate the support and encouragement of my family, namely my wife Lynn, my parents Lawrence and Beth Ann Adams, and my wife's parents, Ren and Cathy Schwartz. I am thankful for friends throughout my schooling and from the Boys J.I.M. Club of America for their continual support.

Finally, I would like to thank my Creator, without whom none of this would be possible. 


\section{TABLE OF CONTENTS}

\section{Page}

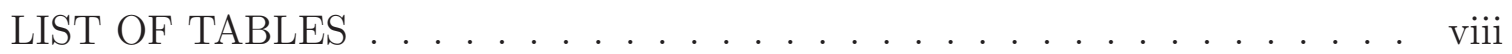

LIST OF FIGURES . . . . . . . . . . . . . . . . . . ix

\section{CHAPTER}

I. INTRODUCTION . . . . . . . . . . . . . . . . . . 1

II. A REVIEW OF INTEGER-ORDER SYSTEMS . . . . . . . . . . . 6

2.1 Derivatives and Integrals . . . . . . . . . . . . . . . . . 6

2.2 The Laplace Transform . . . . . . . . . . . . . . . . . . . . . . 8

2.3 Differential Equations and Transfer Functions . . . . . . . . . . . . 10

2.4 Examples.............................. 14

2.5 Conclusions . . . . . . . . . . . . . . . . . . . . . . 20

III. FRACTIONAL-ORDER CALCULUS . . . . . . . . . . . . . . 21

3.1 Special Functions . . . . . . . . . . . . . . . . . 21

3.2 Definitions ........................ 23

3.3 The Laplace Transform of Fractional-Order Differintegrals . . . . . 26

3.4 Fractional-Order Systems . . . . . . . . . . . . . . 27

3.5 Approximation of Fractional-Order Operators . . . . . . . . . 36

3.6 Initialization of Fractional-Order Systems . . . . . . . . . . . . . . 38 
3.7 Examples . . . . . . . . . . . . . . . . . . . . . 41

3.8 Conclusions . . . . . . . . . . . . . . . . . . . . . . . . . 49

IV. CONJUGATE-ORDER OPERATORS . . . . . . . . . . . . 51

4.1 Complex-Order Differintegrals . . . . . . . . . . . . . 51

4.2 Conjugate-Order Differintegrals . . . . . . . . . . 53

4.3 Conjugate-Order Systems . . . . . . . . . . . . . . . 55

4.4 Stable, Causal Conjugate-Order Systems _ . . . . . . . . . 60

4.5 Conclusions . . . . . . . . . . . . . . . . . . . 102

V. OPERATORS ON A HILBERT SPACE . . . . . . . . . . . 103

5.1 Hilbert Spaces . . . . . . . . . . . . . . . . . . . . . . 104

5.2 Bounded Operators . . . . . . . . . . . . . . . . 107

5.3 Compact Operators . . . . . . . . . . . . . . . . . . . . 109

5.4 The Spectral Theorem for Compact Hermitian Operators ... . 111

5.5 Operators on $L_{2}(\mathbb{R}) \ldots \ldots \ldots \ldots \ldots \ldots \ldots$

5.6 Integral Operators on $L_{2}(\mathbb{R}) \ldots \ldots \ldots \ldots$

5.7 Estimation of Eigenvalues for Operators on a Hilbert Space . . . 135

5.8 Examples . . . . . . . . . . . . . . . . . . . . . . . . . . 141

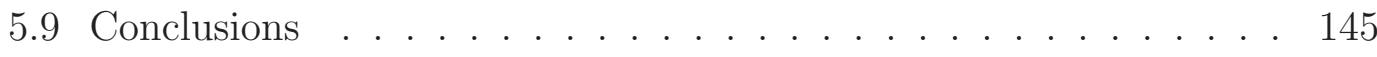

VI. HANKEL OPERATORS . . . . . . . . . . . . . . 147

6.1 Representations of Hankel Operators . . . . . . . . . . . . . 147

6.2 Well-Known Results Concerning Hankel Operators . . . . . . . . 153 
6.3 Integral-Operator Hankel Operators for Fractional-Order Systems . . . . . . . . . . . . . . . 156

6.4 Hankel Operator Equivalences . . . . . . . . . . . . . . . 160

6.5 Compactness of the Hankel Operator for a Class of FractionalOrder Systems . . . . . . . . . . . . . . . . . . 162

6.6 Examples . . . . . . . . . . . . . . . . . . . . 173

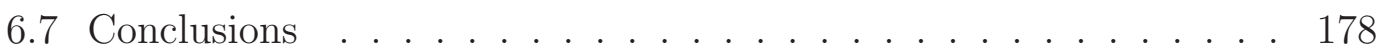

VII. ESTIMATING THE HANKEL SINGULAR VALUES OF FRACTIONAL-ORDER SYSTEMS . . . . . . . . . . . . 179

7.1 Methodology . . . . . . . . . . . . . . . . . . . . . 179

7.2 Hankel Singular Values for Some Real-Order Systems … . . . 182

7.3 Examples . . . . . . . . . . . . . . . . . . . . . . 209

7.4 Conclusions . . . . . . . . . . . . . . . . . . 215

VIII. ESTIMATION OF THE HANKEL NORM FOR A CLASS OF CONJUGATE-ORDER SYSTEMS . . . . . . . . . . . 216

8.1 Preliminary Considerations . . . . . . . . . . . . . . 216

8.2 Estimation . . . . . . . . . . . . . . . . . . 218

8.3 Conclusions . . . . . . . . . . . . . . . . . . . 225

IX. CONCLUSIONS AND FUTURE WORK . . . . . . . . . 226 BIBLIOGRAPHY . . . . . . . . . . . . . . . . . . . . 229

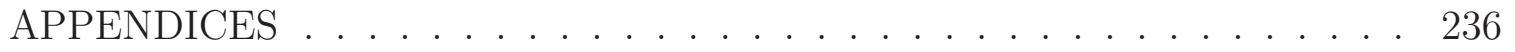

APPENDIX A. LIST OF SYMBOLS . . . . . . . . . . 237

APPENDIX B. MATHEMATICA CODE . . . . . . . . . . . . 241 


\section{LIST OF TABLES}

Table

Page

$3.12 .5 \mathrm{~dB}$-Approximations for $s^{\frac{-k}{10}}$ over $\omega \in[0.01,100]$ for $1 \leq k \leq 9 . \ldots 38$

6.1 Hankel Operator Notation. . . . . . . . . . . . . . . . . . . 151

7.1 Hankel Singular Value Bounds for $\frac{a}{s^{\frac{1}{2}}+a} \ldots \ldots$. . . . . . . . . . . . 186

7.2 Hankel Singular Value Estimates $\hat{\sigma}_{k}$ and Upper Bounds for ResistorTerminated Semi-Infinite Line Using an 800-Dimensional Subspace. . . 210

7.3 Hankel Singular Value Estimates $\hat{\sigma}_{k}$ and Upper Bounds for ResistorTerminated Semi-Infinite Line Using a 1,200-Dimensional Subspace. . . 211

7.4 Minimum Percent Error of Finite-Rank Approximations to the Resistor-Terminated and Inductor-Terminated Semi-Infinite Lines. . . . 212

7.5 Hankel Singular Value Estimates $\hat{\sigma}_{k}$ and Upper Bounds for InductorTerminated Semi-Infinite Line Using a 400-Dimensional Subspace. . . . 214 


\section{LIST OF FIGURES}

Figure $\quad$ Page

$2.1 \quad$ Impulse Response for $G(s)=\frac{-2 s}{s^{2}+s+\frac{1}{4}} \cdot \ldots \ldots \ldots \ldots \ldots$

2.2 Circuit Symbol for Semi-Infinite Line. . . . . . . . . . . . . . . . . 19

3.1 Resistor-Terminated Semi-Infinite Line. . . . . . . . . . . . . . . . . 42

3.2 Impulse Response for Resistor-Terminated Semi-Infinite Line with $\frac{A}{R}=\frac{1}{2}$ (dotted), $\frac{A}{R}=1$ (dashed), and $\frac{A}{R}=2$ (solid). . . . . . . 44

3.3 Magnitude and Phase Responses for a Resistor-Terminated SemiInfinite Line with $\frac{A}{R}=1$ (solid) and a Fifth-Order Approximation (dashed) . . . . . . . . . . . . . . . . . 45

3.4 Inductor-Terminated Semi-Infinite Line. . . . . . . . . . . . . . 46

3.5 Impulse Response for Inductor-Terminated Semi-Infinite Line with $\frac{A}{L}=\frac{1}{2}$ (dotted), $\frac{A}{L}=1$ (dashed), and $\frac{A}{L}=2$ (solid). . . . . . . . 48

3.6 Magnitude and Phase Responses for a Resistor-Terminated SemiInfinite Line with $\frac{A}{L}=1$ (solid) and a Fifth-Order Approximation (dashed) . . . . . . . . . . . . . . . . .

4.1 Set of $q=u+i v$ for which the system $G(s)=\frac{r}{s^{q}-\left(e^{i \pi}\right)}+\frac{\bar{r}}{s^{\bar{q}}-\left(e^{-i \pi}\right)}$ is stable and causal. . . . . . . . . . . . . . . . . 70

4.2 Set of $q=u+i v$ for which the system $G(s)=\frac{r}{s^{q}-\left(2 e^{i \pi}\right)}+\frac{\bar{r}}{s^{\bar{q}}-\left(2 e^{-i \pi}\right)}$ is stable and causal. . . . . . . . . . . . . . . . . . . 71

4.3 Set of $q=u+i v$ for which the system $G(s)=\frac{r}{s^{q}-\left(4 e^{i \pi}\right)}+\frac{\bar{r}}{s^{\bar{q}}-\left(4 e^{-i \pi}\right)}$ is stable and causal. . . . . . . . . . . . . . . . . . 72

4.4 Set of $q=u+i v$ for which the system $G(s)=\frac{r}{s^{q}-\left(8 e^{i \pi}\right)}+\frac{\bar{r}}{s^{\bar{q}}-\left(8 e^{-i \pi}\right)}$ is stable and causal. . . . . . . . . . . . . . . . . . . 
4.5 Set of $q=u+i v$ for which the system $G(s)=\frac{r}{s^{q}-\left(16 e^{i \pi}\right)}+\frac{\bar{r}}{s^{\bar{q}}-\left(16 e^{-i \pi}\right)}$ is stable and causal. . . . . . . . . . . . . . . . . . . . 74

4.6 Set of $q=u+i v$ for which the system $G(s)=\frac{r}{s^{q}-\left(32 e^{i \pi}\right)}+\frac{\bar{r}}{s^{\bar{q}}-\left(32 e^{-i \pi}\right)}$ is stable and causal. . . . . . . . . . . . . . . . . . . 75

4.7 Set of $q=u+i v$ for which the system $G(s)=\frac{r}{s^{q}-\left(64 e^{i \pi}\right)}+\frac{\bar{r}}{s^{\bar{q}}-\left(64 e^{-i \pi}\right)}$ is stable and causal. . . . . . . . . . . . . . . . . . . . . 76

4.8 Set of $q=u+i v$ for which the system $G(s)=\frac{r}{s^{q}-\left(128 e^{i \pi}\right)}+\frac{\bar{r}}{s^{\bar{q}}-\left(128 e^{-i \pi}\right)}$ is stable and causal. . . . . . . . . . . . . . . . . . . . . . . 77

4.9 Set of $q=u+i v$ for which the system $G(s)=\frac{r}{s^{q}-\left(256 e^{i \pi}\right)}+\frac{\bar{r}}{s^{\bar{q}}-\left(256 e^{-i \pi}\right)}$ is stable and causal. . . . . . . . . . . . . . . . . . . . . 78

4.10 Set of $q=u+i v$ for which the system $G(s)=\frac{r}{s^{q}-\left(512 e^{i \pi}\right)}+\frac{\bar{r}}{s^{\bar{q}}-\left(512 e^{-i \pi}\right)}$ is stable and causal. . . . . . . . . . . . . . . . . . . . . . . 79

4.11 Set of $q=u+i v$ for which the system $G(s)=\frac{r}{s^{q}-\left(1024 e^{i \pi}\right)}+\frac{\bar{r}}{s^{\bar{q}}-\left(1024 e^{-i \pi}\right)}$ is stable and causal. . . . . . . . . . . . . . . . . . . 80

4.12 Set of $q=u+i v$ for which the system $G(s)=\frac{r}{s^{q}-\left(2048 e^{i \pi}\right)}+\frac{\bar{r}}{s^{\bar{q}}-\left(2048 e^{-i \pi}\right)}$ is stable and causal. . . . . . . . . . . . . . . . . . . . . 81

4.13 Set of $q=u+i v$ for which the system $G(s)=\frac{r}{s^{q}-\left(4096 e^{i \pi}\right)}+\frac{\bar{r}}{s^{\bar{q}}-\left(4096 e^{-i \pi}\right)}$ is stable and causal. . . . . . . . . . . . . . . . . .

4.14 Set of $q=u+i v$ for which the system $G(s)=\frac{r}{s^{q}-\left(4096 e^{i \frac{\pi}{2}}\right)}+$ $\frac{\bar{r}}{s^{\bar{q}}-\left(4096 e^{-i \frac{\pi}{2}}\right)}$ is stable and causal. . . . . . . . . . . . . . . . .

4.15 Set of $q=u+i v$ for which the system $G(s)=\frac{r}{s^{q}-\left(4096 e^{i 0}\right)}+\frac{\bar{r}}{s^{\bar{q}}-\left(4096 e^{-i 0}\right)}$ is stable and causal. . . . . . . . . . . . . . . . . . . .

4.16 Set of $q=u+i v$ for which the system $G(s)=\frac{r}{s^{q}-\left(4096 e^{-i \frac{\pi}{2}}\right)}+$ $\frac{\bar{r}}{s^{\bar{q}}-\left(4096 e^{i \frac{\pi}{2}}\right)}$ is stable and causal. . . . . . . . . . . . . . . . . .

4.17 Set of $a=|a| e^{i \operatorname{Arg}(a)}$ for which the system $G(s)=\frac{r}{s^{\frac{1}{10}(1+i)}-a}+$ $\frac{\bar{r}}{s^{\frac{1}{10}(1-i)}-\bar{a}}$ is stable and causal.

4.18 Set of $a=|a| e^{i \operatorname{Arg}(a)}$ for which the system $G(s)=\frac{r}{s^{\frac{1}{5}(1+i)}-a}+\frac{\bar{r}}{s^{\frac{1}{5}(1-i)}-\bar{a}}$ is stable and causal. . . . . . . . . . . . . . . . . . . . .

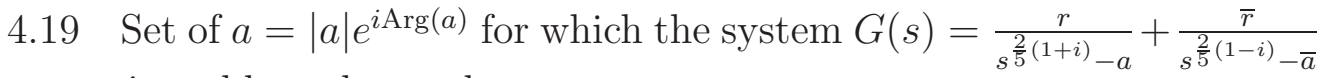
is stable and causal. . . . . . . . . . . . . . . . . . . 
4.20 Set of $a=|a| e^{i \operatorname{Arg}(a)}$ for which the system $G(s)=\frac{r}{s^{\frac{1}{2}(1+i)}-a}+\frac{\bar{r}}{s^{\frac{1}{2}(1-i)}-\bar{a}}$ is stable and causal. . . . . . . . . . . . . . . . . . .

4.21 Set of $a=|a| e^{i \operatorname{Arg}(a)}$ for which the system $G(s)=\frac{r}{s^{\frac{3}{5}(1+i)}-a}+\frac{\bar{r}}{s^{\frac{3}{5}(1-i)}-\bar{a}}$ is stable and causal. . . . . . . . . . . . . . . . . . . .

4.22 Set of $a=|a| e^{i \operatorname{Arg}(a)}$ for which the system $G(s)=\frac{r}{s^{\frac{4}{5}(1+i)}-a}+\frac{\bar{r}}{s^{\frac{4}{5}(1-i)}-\bar{a}}$ is stable and causal. . . . . . . . . . . . . . . . . . . .

4.23 Set of $a=|a| e^{i \operatorname{Arg}(a)}$ for which the system $G(s)=\frac{r}{s^{0.99(1+i)}-a}+$ $\frac{\bar{r}}{s^{0.99(1-i)}-\bar{a}}$ is stable and causal. . . . . . . . . . . . . 96

4.24 Set of $a=|a| e^{i \operatorname{Arg}(a)}$ for which the system $G(s)=\frac{r}{s^{\left(1+\frac{4 i}{5}\right)}-a}+\frac{\bar{r}}{s^{\left(1-\frac{4 i}{5}\right)}-\bar{a}}$ is stable and causal. . . . . . . . . . . . . . . . . . . . . . . . . 97

4.25 Set of $a=|a| e^{i \operatorname{Arg}(a)}$ for which the system $G(s)=\frac{r}{s^{\left(1+\frac{3 i}{5}\right)}-a}+\frac{\bar{r}}{s^{\left(1-\frac{3 i}{5}\right)}-\bar{a}}$ is stable and causal. . . . . . . . . . . . . . . . . . . . . . . . . . 98

4.26 Set of $a=|a| e^{i \operatorname{Arg}(a)}$ for which the system $G(s)=\frac{r}{s^{\left(1+\frac{2 i}{5}\right)}-a}+\frac{\bar{r}}{s^{\left(1-\frac{2 i}{5}\right)}-\bar{a}}$ is stable and causal. . . . . . . . . . . . . . . . . . . . . . . . . . . 99

4.27 Set of $a=|a| e^{i \operatorname{Arg}(a)}$ for which the system $G(s)=\frac{r}{s^{\left(1+\frac{i}{5}\right)}-a}+\frac{\bar{r}}{s^{\left(1-\frac{i}{5}\right)}-\bar{a}}$ is stable and causal. . . . . . . . . . . . . . . . . . . 100

4.28 Set of $a=|a| e^{i \operatorname{Arg}(a)}$ for which the system $G(s)=\frac{r}{s^{1}-a}+\frac{\bar{r}}{s^{1}-\bar{a}}$ is stable and causal. . . . . . . . . . . . . . . . . . . . . 101

7.1 Estimates (-) and Error Bounds (- -) of $\sigma_{1}$ and $\sigma_{2}$ for $G(s)=\frac{-2 s}{s^{2}+s+\frac{1}{4}} \ldots \quad$. 185

7.2 Estimates (-) and Error Bounds (- -) for $\sigma_{1}\left(\frac{1}{s^{q}+1}\right)$ for $q \in(0,2) . \ldots 188$

7.3 Estimates (-) and Error Bounds (- - ) for $\sigma_{1}\left(\frac{1}{s^{q}+1}\right)$ for $q \in(0,1] \ldots 189$

7.4 Estimates (-) and Error Bounds (- -) for $\sigma_{2}\left(\frac{1}{s^{q}+1}\right)$ for $q \in(0,2) . \quad \ldots . . \quad 190$

7.5 Estimates (-) and Error Bounds (- -) for $\sigma_{3}\left(\frac{1}{s^{q}+1}\right)$ for $q \in(0,2)$. . . . 191

7.6 Estimates (-) and Error Bounds (- -) for $\sigma_{4}\left(\frac{1}{s^{q}+1}\right)$ for $q \in(0,2) . \quad$. . . 192

7.7 Estimates (-) and Error Bounds (- -) for $\sigma_{5}\left(\frac{1}{s^{q}+1}\right)$ for $q \in(0,2) . \quad \ldots \quad$. 193

7.8 Estimates (-) and Error Bounds (- -) for $\sigma_{6}\left(\frac{1}{s^{q}+1}\right)$ for $q \in(0,2) . \ldots 194$ 
7.9 Estimates (-) and Error Bounds (- -) for $\sigma_{7}\left(\frac{1}{s^{q}+1}\right)$ for $q \in(0,2) . \quad$. . . . 195

7.10 Estimates (-) and Error Bounds (- -) for $\sigma_{8}\left(\frac{1}{s^{q}+1}\right)$ for $q \in(0,2) . \quad \ldots \quad 196$

7.11 Estimates (-) and Error Bounds (- -) for $\sigma_{9}\left(\frac{1}{s^{q}+1}\right)$ for $q \in(0,2) . \ldots 197$

7.12 Estimates (-) and Error Bounds (- -) for $\sigma_{10}\left(\frac{1}{s^{q}+1}\right)$ for $q \in(0,2) . . . . \quad 198$

7.13 Estimates (-) and Bounds (- -) on the Percent Error in Hankel Norm Between $G(s)$ and One-Dimensional Approximation. . . . . . . . . . . 200

7.14 Estimates (-) and Bounds (- -) on the Percent Error in Hankel Norm Between $G(s)$ and Two-Dimensional Approximation. . . . . . . . . . . 201

7.15 Estimates (-) and Bounds (- -) on the Percent Error in Hankel Norm Between $G(s)$ and Three-Dimensional Approximation. . . . . . . . . . . 202

7.16 Estimates (-) and Bounds (- -) on the Percent Error in Hankel Norm Between $G(s)$ and Four-Dimensional Approximation. . . . . . . . . . 203

7.17 Estimates (-) and Bounds (- -) on the Percent Error in Hankel Norm Between $G(s)$ and Five-Dimensional Approximation. . . . . . . . . . . 204

7.18 Estimates (-) and Bounds (- -) on the Percent Error in Hankel Norm Between $G(s)$ and Six-Dimensional Approximation. . . . . . . . . . . 205

7.19 Estimates (-) and Bounds (- -) on the Percent Error in Hankel Norm Between $G(s)$ and Seven-Dimensional Approximation. . . . . . . . . . . 206

7.20 Estimates (-) and Bounds (- -) on the Percent Error in Hankel Norm Between $G(s)$ and Eight-Dimensional Approximation. . . . . . . . . . . 207

7.21 Estimates (-) and Bounds (- -) on the Percent Error in Hankel Norm Between $G(s)$ and Nine-Dimensional Approximation. . . . . . . . . . . 208

8.1 Set of $q=u+i v$ for which $G(s)=\frac{r}{s^{q}-\left(e^{i \pi}\right)}+\frac{\bar{r}}{s^{\bar{q}}-\left(e^{-i \pi}\right)}$ corresponds to a stable, causal system. . . . . . . . . . . . . . . . . . 217

8.2 Set of $q=u+i v$ for which the Hankel norm $G(s)=\frac{r}{s^{q}-\left(e^{i \pi}\right)}+\frac{\bar{r}}{s^{\bar{q}}-\left(e^{-i \pi}\right)}$ is calculated. . . . . . . . . . . . . . . . . . . . . . . . . . . 219

8.3 Estimates of the Hankel norm of $G(s)$ with $q \in \mathbb{C}$ such that $0 \leq$

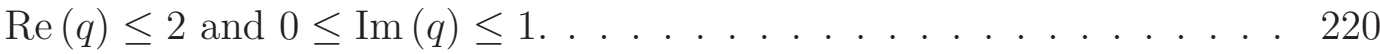

8.4 Contour Plot of the Estimates of the Hankel norm of $G(s)$ with $q \in \mathbb{C}$ such that $0 \leq \operatorname{Re}(q) \leq 2$ and $0 \leq \operatorname{Im}(q) \leq 1 . \ldots . . . . .221$ 
8.5 Estimates of the Hankel norm of $G(s)$ with $q \in \mathbb{C}$ such that $0 \leq$ $\operatorname{Re}(q) \leq 2$ and $-1 \leq \operatorname{Im}(q) \leq 1 \ldots \ldots \ldots \ldots . \ldots \ldots 222$

8.6 Contour Plot of the Estimates of the Hankel norm of $G(s)$ with $q \in \mathbb{C}$ such that $0 \leq \operatorname{Re}(q) \leq 2$ and $-1 \leq \operatorname{Im}(q) \leq 1 \ldots \ldots 223$

8.7 Contour Plot of the Estimates of the Hankel norm of $G(s)$ with $q \in \mathbb{C}$ such that $-2 \leq \operatorname{Re}(q) \leq 2$ and $-1 \leq \operatorname{Im}(q) \leq 1 \ldots \ldots . . \ldots 224$ 


\section{CHAPTER I}

\section{INTRODUCTION}

The fractional-order calculus generalizes the order of differentiation and integration from integers to all complex numbers. The subject traditionally has been said to originate from the notation of Leibnitz for the $n^{\text {th }}$ derivative, $\frac{d^{n} f}{d t^{n}}$. Rather than being limited to $n^{\text {th }}$-order iterated derivatives and integrals with $n \in \mathbb{N}$, with the fractional-order calculus it is possible to have half-order, three-quarter-order, $\pi$-order, or $\sqrt{-1}$-order derivatives. As the possible orders are more than just fractions, the fractional-order calculus might more accurately be called the calculus of non-integer order. However, the term fractional-order calculus is an historic one, and will be used throughout the rest of this work.

Systems with fractal physical arrangements or spatially distributed variables, which cannot easily be modeled using the integer-order calculus, may be represented compactly by a fractional-order transfer function. Some of the applications of fractional-order calculus include systems with both energy dissipation and energy storage behavior such as viscoelasticity [12, 27, 37, 79], diffusive transport $[57,25,42,55]$, electrochemical processes and corrosion [62, 63, 64], fluid flow $[22,23,17]$, rheology $[85,13,11,38,59]$, and dynamic processes in porous and selfsimilar structures [70, 71]. 
Little attention has been given to Hankel operators for fractional-order systems. The Hankel operator, which maps the past inputs of a given system to its future outputs, is a valuable tool in system analysis, model reduction, and controller synthesis for integer-order systems. For these uses the spectral decomposition of the Hankel operator of the system must be well-understood. In the case of fractionalorder systems, this requires a function analytic approach [24] as opposed to the more convenient state-space approach that is available for systems of integer order [86].

The spectral decomposition of fractional-order operators has had very limited study [28]. What study has occurred in this area was done using weighted $L_{2}$ spaces. It is the aim of this thesis to obtain estimates on the singular values of the Hankel operators for a variety of fractional-order systems in the non-weighted space $L_{2}(\mathbb{R})$. The largest of these singular values is the Hankel norm, which is the maximum ratio of the energy that can be retrieved from the system in the future to the energy put into the system in the past. The $(k+1)^{\text {th }}$ singular value represents a lower bound on the Hankel-norm error between a system and a $k^{\text {th }}$-order approximation to the system [75].

This dissertation presents the background and theory necessary to consider the spectral decomposition of the Hankel operator for fractional-order systems. An algorithm for estimating the Hankel singular values of a fractional-order system using four isomorphic Hilbert spaces is presented. The first ten Hankel singular values for systems of the form $G(s)=\frac{1}{s^{q}+1}$ for $q \in(0,2)$ are estimated with bounds on the error of the estimation. Also estimated are the Hankel norms for systems of the 
form $G(s)=\frac{1}{s^{q}+1}+\frac{1}{s^{\bar{q}}+1}$ for various $q \in \mathbb{C}$. The estimates are calculated in $\ell_{2}(\mathbb{N})$. The bounds on the error of the estimates, which are calculated in $H_{2}(\mathbb{D})$, are more difficult to compute than the estimates themselves. The estimates and the bounds are calculated for the first ten Hankel singular values of two physical fractional-order systems, the resistor-terminated and inductor-terminated semi-infinite lines. The bounds indicate that the relative errors in the Hankel singular value estimates are each less $17 \%$.

To begin, Chapter 2 presents a review of integer-order systems. Some of the results for differentiation and integration are used later in formulating the definitions of fractional differintegral operators. Also presented are results on bilateral Laplace transforms, differential equations, transfer functions, and convolution operators, which will be used in the analysis of fractional-order systems.

Chapter 3 begins the discussion on fractional-order operators by defining the gamma function and the Mittag-Leffler Function. The Grünwald-Leitnikov, RiemannLiouville, and Caputo definitions of the non-integer-order differintegral are given. The Laplace transform, fractional-order differential equations, and fractional-order transfer functions are discussed. Fractional-order impulse responses, approximations of fractional-order systems, and initialization of fractional-order systems are highlighted.

Chapter 4 discusses the extension of fractional-order systems to conjugateorder systems. First, pairs of complex-conjugate complex-order differintegrals are considered. It is demonstrated that this differintegral pairing has a real-valued impulse response. The fundamental, linear conjugate-order system $G(s)=\frac{r}{s^{q}-a}+\frac{\bar{r}}{s^{\bar{q}}-\bar{a}}$ 
is considered in detail. The real-valued impulse response for $G(s)$ is developed. Also the pole locations of $G(s)$ are shown. For a given $a$, the set of $q$ for which $G(s)$ corresponds to a stable, causal system are developed, and examples for several $a$ are given. Similarly, for a given $q$, the set of $a$ for which $G(s)$ corresponds to a stable, causal system are developed, and examples for various $q$ are given.

Chapter 5 contains a background on Hilbert-space operators. The most important result is the Spectral Theorem for Compact Hermitian Operators. Chapter 5 also describes linear integral operators on $L_{2}(\mathbb{R})$, which include the convolution operators. The use of convolution operators in integer-order systems is described. The chapter concludes with a method for estimating the eigenvalues of a compact Hermitian operator on a Hilbert space.

Chapter 6 discusses Hankel operators. Representations as operators on the Hilbert spaces $L_{2}\left(\mathbb{R}_{+}\right), H_{2}\left(\mathbb{C}^{+}\right), H_{2}(\mathbb{D})$, and $\ell_{2}(\mathbb{N})$ are given. The Nehari problem and its solution are given. The applications of Hankel operators to both the model-reduction problem and to $H_{\infty}$-control are also discussed. Hankel operators for fractional-order systems are considered, and it is shown that such Hankel operators have infinite rank. The conditions under which the Hankel operator for systems of the form $G(s)=\frac{r}{s^{q}-a}+\frac{\bar{r}}{s^{q}-\bar{a}}$ is compact are also shown.

In Chapter 7 an algorithm to estimate the Hankel singular values for a fractional-order system is presented. The first ten Hankel singular values for the class of systems $\frac{a}{s^{\frac{1}{2}}-a}$ are determined for several $a$. The first ten Hankel singular values for the class of systems $\frac{1}{s^{q}+1}$ are determined for a variety of $q \in(0,2)$. 
Chapter 8 presents estimates of the Hankel norm for a class of conjugateorder systems. The set of $q$ for which $\frac{\frac{1}{2}}{s^{q}+1}+\frac{\frac{1}{2}}{s^{q}+1}$ corresponds to a compact operator is given. Estimates of the Hankel norms for this class of conjugate-order systems are then given.

Chapter 9 considers the conclusions that may be drawn in this dissertation. Also presented is a discussion of future work. 


\section{CHAPTER II}

\section{A REVIEW OF INTEGER-ORDER SYSTEMS}

The background material begins with a review of linear, time-invariant, integer-order systems. We give definitions of iterated derivatives and integrals, which will later be generalized, yielding the definitions of the fractional-order differintegral. The bilateral Laplace transform, as well as ordinary integer-order differential equations and integer-order transfer functions are discussed to provide a familiar context for comparison with ordinary fractional-order differential equations and fractional-order transfer functions. Finally, in this chapter the representation of an integer-order system as a convolution operator is discussed.

\subsection{Derivatives and Integrals}

The integer-order or ordinary calculus begins with the definition of a derivative. The first derivative is defined by

$$
\frac{d}{d t} f(t)=\lim _{\Delta t \rightarrow 0} \frac{f(t)-f(t-\Delta t)}{\Delta t}
$$


if this limit exists. For any $n \in \mathbb{N}$, this definition may be iterated, yielding

$$
\frac{d^{n}}{d t^{n}} f(t)=\lim _{\Delta t \rightarrow 0}(\Delta t)^{-n} \sum_{k=0}^{n}(-1)^{k}\left(\frac{n !}{k !(n-k) !}\right) f(t-k \Delta t)
$$

For convenience, we denote the $n^{\text {th }}$-order derivative for a function defined on the interval $[a, b]$ by

$$
\frac{d^{n}}{d t^{n}} f(t)={ }_{a} d_{t}^{n} f(t)
$$

for all $t \in(a, b]$. This notation is used for fractional-order derivatives, so for consistency it will be retained for integer-order derivatives, even though an integer-order derivative does not require the left-hand endpoint $a$ to be specified. The subscript $t$, for the causal differential operator, denotes the variable with respect to which the differentiation occurs.

The notation used in Equation 2.3 can be extended to apply to iterated integrals as

$$
\begin{aligned}
{ }_{a} d_{t}^{-1} f(t) & =\int_{a}^{t} f(\tau) d \tau \\
{ }_{a} d_{t}^{-2} f(t) & =\int_{a}^{t} \int_{a}^{\xi} f(\tau) d \tau d \xi
\end{aligned}
$$

and so forth.

Without loss of generality, we let the lower limit of the integrals be zero. According to Fubini's theorem, the order of integration in Equation 2.5 can be reversed, 
provided that $\int_{0}^{\infty}|f(t)| d t<\infty$. This yields the result

$$
\begin{aligned}
{ }_{0} d_{t}^{-2} f(t) & =\int_{0}^{t} \int_{0}^{\xi} f(\tau) d \tau d \xi \\
& =\int_{0}^{t} \int_{\tau}^{t} f(\tau) d \xi d \tau \\
& =\int_{0}^{t} f(\tau) \int_{\tau}^{t} d \xi d \tau \\
& =\int_{0}^{t} f(\tau)(t-\tau) d \tau .
\end{aligned}
$$

The formula can be iterated, resulting in

$$
{ }_{0} d_{t}^{-n} f(t)=\frac{1}{(n-1) !} \int_{0}^{t}(t-\tau)^{n-1} f(\tau) d \tau
$$

which is Cauchy's formula for iterated integrals. This formula will be generalized in Chapter 3 to include non-integer values of $n$.

\subsection{The Laplace Transform}

The bilateral Laplace transform of a function $f(t)$ denoted by $F(s)$ is given by

$$
F(s)=\mathcal{L}\{f(t)\}=\int_{-\infty}^{\infty} f(t) e^{-s t} d t
$$

if the integral converges for some values of $s$ in the complex plane [67]. The set of $s$ for which the integral converges is called the region of convergence for the Laplace 
transform and is denoted by $\mathcal{R}$. The following theorem gives a result concerning the region of convergence.

Theorem 2.2.1 Let $F(s)=\mathcal{L}\{f(t)\}$ with region of convergence $\mathcal{R}$. Then $F(s)$ is analytic in $\mathcal{R}$.

Proof See [61].

The following theorem details some properties of the bilateral Laplace transform. One such property involves the convolution of two functions. Recall the definition of convolution.

Definition The convolution of two functions, $f(t)$ and $g(t)$, defined on $\mathbb{R}$ is denoted $(f * g)(t)$ and is defined by

$$
(f * g)(t)=\int_{-\infty}^{\infty} f(t-\tau) g(\tau) d \tau
$$

Theorem 2.2.2 (Properties of the Bilateral Laplace Transform) Let $F(s)=$ $\mathcal{L}\{f(t)\}$. Then,

1. $\mathcal{L}\left\{f\left(t-t_{0}\right)\right\}=e^{-s t_{0}} F(s)$

2. $\mathcal{L}\{f(a t)\}=\frac{1}{|a|} F\left(\frac{s}{a}\right)$

3. $\mathcal{L}\left\{\left(f_{1} * f_{2}\right)(t)\right\}=F_{1}(s) F_{2}(s)$

4. $\mathcal{L}\left\{\frac{d}{d t} f(t)\right\}=s F(s)$ 
5. $\mathcal{L}\left\{\int_{-\infty}^{t} f(\tau) d \tau\right\}=\frac{1}{s} F(s)$

Proof See [67].

A useful consequence of Part 4 of Theorem 2.2.2 is that the Laplace transform converts a differential equation into an algebraic equation. An analogous property applying to fractional-order derivatives will be introduced in Chapter 3.

\subsection{Differential Equations and Transfer Functions}

Definition An ordinary integer-order linear constant-coefficient differential equation is an equation of the form

$$
\sum_{k=0}^{n} a_{k} \frac{d^{k}}{d t^{k}} x(t)=\sum_{k=0}^{m} b_{k} \frac{d^{k}}{d t^{k}} f(t),
$$

with $a_{n} \neq 0$ and $b_{m} \neq 0$ where $f(t)$ is called the forcing function. Assuming $n \geq m$, $n$ is the order of the differential equation.

Differential equations of this form are linear and time-invariant; that is, if $x_{1}(t)$ and $x_{1}(t)$ are the solutions of the differential equation equation with the forcing functions $f_{1}(t)$ and $f_{2}(t)$, respectively, and $\alpha_{1}, \alpha_{2} \in \mathbb{C}$, then $\alpha_{1} x_{1}(t)+\alpha_{2} x_{2}(t)$ is the solution of the differential equation with forcing function $\alpha_{1} f_{1}(t)+\alpha_{2} f_{2}(t)$ and $x_{1}\left(t+t_{0}\right)$ is the solution of the differential equation with forcing function $f\left(t+t_{0}\right)$ for 
any $t_{0} \in \mathbb{R}$. Systems that satisfy these two properties are called linear time-invariant systems. Only linear time-invariant systems will be considered in this dissertation.

Using the differentiation property of the Laplace transform, Equation 2.9 can be transformed into the equation,

$$
\sum_{k=0}^{n} a_{k} s^{k} X(s)=\sum_{k=0}^{m} b_{k} s^{k} F(s)
$$

where $X(s)=\mathcal{L}\{x(t)\}$ and $F(s)=\mathcal{L}\{f(t)\}$. The transfer function $G(s)$ is defined by

$$
G(s)=\frac{X(s)}{F(s)}=\frac{\sum_{k=0}^{m} b_{k} s^{k}}{\sum_{k=0}^{n} a_{k} s^{k}} .
$$

The system impulse response, denoted as $\tilde{g}(t)$, is defined by

$$
\tilde{g}(t)=\mathcal{L}^{-1}\{G(s)\}
$$

Let $\mathbb{R}[s]$ denote the set of all polynomials in $s$ with coefficients in $\mathbb{R}$ and let $\mathbb{R}(s)$ denote the set of all ratios of elements in $\mathbb{R}[s]$. Define $A(s)=\sum_{k=0}^{n} a_{k} s^{k}$ and $B(s)=\sum_{k=0}^{m} b_{k} s^{k}$ with real coefficients. Note that $A(s), B(s) \in \mathbb{R}[s]$ and that $\frac{B(s)}{A(s)}=G(s) \in \mathbb{R}(s)$. By the Fundamental Theorem of Algebra, $A(s)$ has $n$ roots, and $B(s)$ has $m$ roots, and these roots are either real or occur in complex-conjugate pairs. Definition The roots of $A(s)$ are the poles of $G(s)$, and the roots of $B(s)$ are the zeroes of $G(s)$. Denote the poles by $\left\{p_{k}\right\}_{k=1}^{n}$ and the zeroes by $\left\{z_{k}\right\}_{k=1}^{m}$. 
Partial-fraction expansion can be used on $G(s)$ to obtain a finite sum of firstorder terms in $\mathbb{C}(s)$. Let $p_{k}$ be the $k^{\text {th }}$ pole of $G(s)$, with multiplicity $m_{k}$. Then a transfer function for a system can be written as

$$
G(s)=\frac{\sum_{k=0}^{m} b_{k} s^{k}}{\sum_{k=0}^{n} a_{k} s^{k}}=\sum_{k=1}^{k_{n}} \sum_{i=1}^{m_{k}} \frac{r_{k_{i}}}{\left(s-p_{k}\right)^{i}}
$$

Note that if a pole or partial-fraction coefficient is complex, its complex conjugate is also a pole or partial-fraction coefficient. Let

$$
\mathcal{U}_{s}(t)= \begin{cases}1, & t \in[0, \infty) \\ 0, & t \in(-\infty, 0)\end{cases}
$$

be the unit step function. Utilizing the Laplace transform pairs,

$$
\begin{aligned}
\mathcal{L}\left\{\frac{t^{m-1} e^{b t}}{(m-1) !} \mathcal{U}_{s}(t)\right\} & =\frac{1}{(s-b)^{m}}, \operatorname{Re}(s)>\operatorname{Re}(b) \\
\mathcal{L}\left\{\frac{t^{m-1} e^{b t}}{(m-1) !} \mathcal{U}_{s}(-t)\right\} & =\frac{1}{(b-s)^{m}}, \operatorname{Re}(s)<\operatorname{Re}(b)
\end{aligned}
$$

given in [61], then the impulse response $\tilde{g}(t)$ for the original differential equation is

$$
\tilde{g}(t)=\sum_{k=1}^{k_{n}} \sum_{i=1}^{m_{k}} r_{k_{i}} \frac{t^{i-1} e^{p_{k} t}}{(i-1) !} \mathcal{U}_{k}(t)
$$

where $\mathcal{U}_{k}(t)= \pm \mathcal{U}_{s}( \pm t)$ depending on the causality and multiplicity of the $k^{\text {th }}$ mode of response. 
We now consider linear, time-invariant, integer-order systems as convolution operators.

\subsubsection{Convolution Operators}

For a given system, or differential equation, $G(s)$ is fixed. Let $\tilde{g}(t)=\mathcal{L}^{-1}\{G(s)\}$, and note that the solution of the differential equation can be written as

$$
x(t)=\mathcal{L}^{-1}\{X(s)\}=\mathcal{L}^{-1}\{G(s) F(s)\}=(\tilde{g} * f)(t)
$$

by the convolution property of Theorem 2.2.2. Hence if $f(t)$ is the input and $x(t)$ the output of a system, then the system can be represented by the equation

$$
x(t)=(\tilde{g} * f)(t)=\int_{-\infty}^{\infty} \tilde{g}(t-\tau) f(\tau) d \tau=(\mathcal{G} f)(t)
$$

where $\mathcal{G}$ denotes the convolution operator. $\mathcal{G}$ is causal if $\tilde{g}(t)=0$ for all $t<0$. $\mathcal{G}$ is anti-causal if $\tilde{g}(t)=0$ for all $t>0$ [24]. The function $\tilde{g}(t-\tau)$ is called the kernel of the convolution operator.

The response $x(t)$ of a system to an input $f(t)$ is given in the Laplace domain as $X(s)=G(s) F(s)$. Recall that $x(t)=(\tilde{g} * f)(t)$ or $x(t)=(\mathcal{G} f)(t)$ as defined by Equation 2.14. For $t>0$, the integral in Equation 2.14 can be broken up as

$$
\begin{aligned}
x(t) & =(\mathcal{G} f)(t)=x_{i c}(t)+x_{f}(t) \\
& =\int_{-\infty}^{0} \tilde{g}(t-\tau) f(\tau) d \tau+\int_{0}^{\infty} \tilde{g}(t-\tau) f(\tau) d \tau .
\end{aligned}
$$


Here, $x_{i c}(t)=\int_{-\infty}^{0} \tilde{g}(t-\tau) f(\tau) d \tau$ is the response of the system to the input applied for $t<0$. This component of the response can be thought of as that which results from initial conditions set up at $t=0$ by the input applied for $t<0$. Thus, we may call it the initial condition response. It is sometimes called the zero-input response. The other component of the solution $x_{f}(t)=\int_{0}^{\infty} \tilde{g}(t-\tau) f(\tau) d \tau$ is the forced response, also called the zero-state response. We will return to convolution operators after we have built some general theory about operators.

\subsection{Examples}

We now consider two examples. The first is an integer-order system, and the second motivates the need for an understanding of fractional-order systems.

\subsubsection{A Second-Order System}

Consider the differential equation

$$
{ }_{-\infty} d_{t}^{2} x(t)+{ }_{-\infty} d_{t}^{1} x(t)+\frac{1}{4} x(t)=-2_{-\infty} d_{t}^{1} f(t)
$$

The transfer function associated with Equation 2.16 is

$$
G(s)=\frac{-2 s}{s^{2}+s+\frac{1}{4}}=\frac{-2 s}{\left(s+\frac{1}{2}\right)^{2}}
$$


and the impulse response of the system, which is shown in Figure 2.1, is

$$
\tilde{g}(t)=\mathcal{L}^{-1}\{G(s)\}=e^{-\frac{t}{2}}(t-2) \mathcal{U}_{s}(t) .
$$

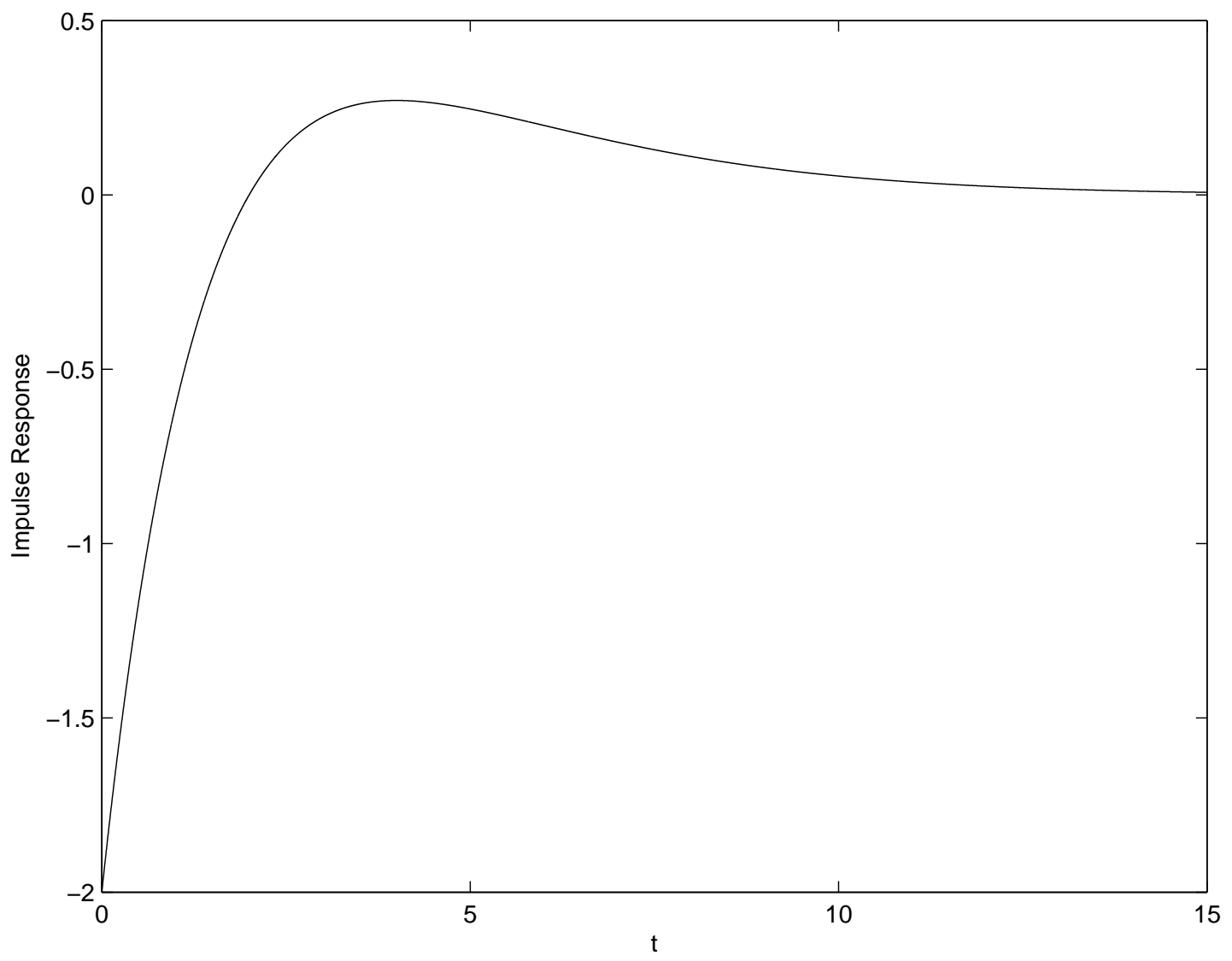

Figure 2.1: Impulse Response for $G(s)=\frac{-2 s}{s^{2}+s+\frac{1}{4}}$.

The convolution operator for this system is

$$
(\mathcal{G} f)(t)=\int_{-\infty}^{\infty} e^{-\frac{t-\tau}{2}}(t-\tau-2) \mathcal{U}_{s}(t-\tau) f(\tau) d \tau
$$


For $t \geq 0$, the initial condition response is

$$
x_{i c}(t)=\int_{-\infty}^{0} e^{-\frac{t-\tau}{2}}(t-\tau-2) f(\tau) d \tau,
$$

and the forced response is

$$
x_{f}(t)=\int_{0}^{t} e^{-\frac{t-\tau}{2}}(t-\tau-2) f(\tau) d \tau .
$$

This system has been represented as a differential equation, a transfer function, and a convolution operator. The initial-condition and forced responses have been shown for this system. We will consider this system as an operator on a Hilbert space in Chapter 5 .

\subsubsection{A Motivating Example}

This development follows that of [30]. Suppose that we have a semi-infinite line with

resistance per unit length $R$ and capacitance per unit length $C$. Define $\alpha=\frac{1}{R C}$. Then the voltage on the line is given by the integer-order partial differential equation,

$$
\frac{\partial}{\partial t} v(x, t)=\alpha \frac{\partial^{2}}{\partial x^{2}} v(x, t)
$$

with $v(0, t)=v_{\text {in }}(t)$ given and $\lim _{x \rightarrow \infty} v(x, t)=0$. We will utilize the bilateral Laplace transform denoted $\mathcal{L}$ to take a function in $t$ to a function in $s$ and the unilateral Laplace transform denoted $\mathcal{L}_{+}$to take a function in $x$ to a function in $p$. 
The current in the line at $x$ is given by

$$
i(x, t)=-\frac{1}{R} \frac{\partial}{\partial x} v(x, t) .
$$

Taking the bilateral Laplace transform in $t$ of Equation 2.22 gives

$$
s V(x, s)=\alpha \frac{\partial^{2}}{\partial x} V(x, s)
$$

with $V(0, s)=V_{\text {in }}(s)=\mathcal{L}\left\{v_{\text {in }}(t)\right\}$ and $\lim _{x \rightarrow \infty} V(x, s)=0$. We will denote $V^{*}(x, s)=$ $\frac{\partial}{\partial x} V(x, s)$ and take the unilateral Laplace transform in $x$ of Equation 2.24, resulting in

$$
\frac{s}{\alpha} \tilde{V}(p, s)=p^{2} \tilde{V}(p, s)-p V(0, s)-V^{*}(0, s)
$$

where $\tilde{V}(p, \cdot)=\mathcal{L}_{+} V(x, \cdot)$. Rearranging and solving for $\tilde{V}(p, s)$ gives

$$
\tilde{V}(p, s)=\frac{p}{p^{2}-\frac{s}{\alpha}} V(0, s)+\frac{1}{p^{2}-\frac{s}{\alpha}} V^{*}(0, s)
$$


The unilateral Laplace transform pairs given by $\frac{p}{p^{2}-a^{2}}=\mathcal{L}_{+}\{\cosh (a x)\}$ and $\frac{a}{p^{2}-a^{2}}=$ $\mathcal{L}_{+}\{\sinh (a x)\}$ are used to obtain

$$
\begin{aligned}
V(x, s) & =\cosh \left(\sqrt{\frac{s}{\alpha}} x\right) V(0, s)+\sqrt{\frac{\alpha}{s}} \sinh \left(\sqrt{\frac{\alpha}{s} x}\right) V^{*}(0, s) \\
& =\frac{1}{2}\left(e^{\sqrt{\frac{s}{\alpha}} x}+e^{-\sqrt{\frac{s}{\alpha}} x}\right) V(0, s)+\frac{1}{2}\left(e^{\sqrt{\frac{s}{\alpha}} x}-e^{-\sqrt{\frac{s}{\alpha}} x}\right)\left(\sqrt{\frac{s}{\alpha}}\right) V^{*}(0, s) \\
& =\frac{1}{2} e^{\sqrt{\frac{s}{\alpha}} x}\left(V(0, s)+\sqrt{\frac{\alpha}{s}} V^{*}(0, s)\right)+\frac{1}{2} e^{-\sqrt{\frac{s}{\alpha}} x}\left(V(0, s)-\sqrt{\frac{\alpha}{s}} V^{*}(0, s)\right)
\end{aligned}
$$

Note that $\sqrt{s}$ is not analytic on the negative real axis $\mathbb{R}_{-}$. Since $\alpha$ is a non-negative real number, $\sqrt{\frac{s}{\alpha}}$ is not analytic on $\mathbb{R}_{-}$. Subsequently, $V(x, s)$ is not analytic with respect to $s$ for $s \in \mathbb{R}_{-}$. Therefore by Theorem 2.2.1, $\mathbb{R}_{-} \not \subset \mathcal{R}$. For $s \in \mathcal{R},|\arg (s)|<$ $\pi$. It follows that for $s \in \mathcal{R},\left|\arg \left(\sqrt{\frac{s}{\alpha}}\right)\right|<\frac{\pi}{2}$. Subsequently, for $s \in \mathcal{R}, \operatorname{Re}\left(\sqrt{\frac{s}{\alpha}}\right)>0$. Since $\lim _{x \rightarrow \infty} V(x, s)=0$, the first term in Equation 2.27 must be zero. Hence,

$$
V(0, s)+\sqrt{\frac{\alpha}{s}} V^{*}(0, s)=0 .
$$

Taking the bilateral Laplace transform of Equation 2.23 gives

$$
I(x, s)=-\frac{1}{R} \frac{\partial}{\partial x} V(x, s)=-\frac{1}{R} V^{*}(x, s) .
$$


Hence, Equation 2.28 can be written as

$$
V(0, s)=R \sqrt{\alpha} \frac{1}{\sqrt{s}} I(0, s) .
$$

We can therefore write the impedance of the lossy semi-infinite line at $x=0$ as

$$
Z(0, s)=\frac{V(0, s)}{I(0, s)}=R \sqrt{\alpha} \frac{1}{\sqrt{s}}=\sqrt{\frac{R}{C}} \frac{1}{\sqrt{s}} .
$$

We define the constant

$$
A=\sqrt{\frac{R}{C}}
$$

and Equation 2.29 can be written as

$$
Z(0, s)=\frac{V(0, s)}{I(0, s)}=\frac{A}{\sqrt{s}}
$$

The circuit symbol for the lossy semi-infinite line is shown in Figure 2.2.

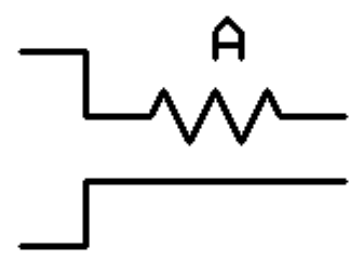

Figure 2.2: Circuit Symbol for Semi-Infinite Line. 
We will see in Chapter 3, that the differential equation associated with Equation 2.30 is

$$
i(0, t)=\frac{1}{A}-\infty d_{t}^{\frac{1}{2}} v(0, t) .
$$

This is an extension of the order of a derivative from integer orders to non-integer orders.

\subsection{Conclusions}

In this chapter we have reviewed integer-order systems. These systems have been represented as differential equations, transfer functions, and convolution operators. An example has been presented that demonstrates these concepts. A motivating example has been given that illustrates the need for an understanding of fractionalorder systems. We now introduce differentiation and integration of non-integer order. 


\section{CHAPTER III}

\section{FRACTIONAL-ORDER CALCULUS}

The fractional calculus is the generalization of the integer-order, or ordinary, calculus to differentiation and integration of non-integer order. Several fairly comprehensive works have been written in recent years on the subject $[66,58,83,77,43]$.

Two special functions that are often used in discussions of fractional-order systems are presented. Next, three common definitions of the fractional-order differintegral and the conditions under which the definitions are equivalent are discussed. The impulse response for a fractional-order system, which is essential to the associated convolution and Hankel operators is derived. We present original material pertaining to the consideration of fractional-order systems of complex order in both the time and frequency domains. A finite-dimensional approximation technique for fractional-order systems is presented. Finally, the problem of proper initialization of fractional-order systems, which is directly related to the Hankel operator, is discussed.

\subsection{Special Functions}

We begin by defining two special functions, the gamma function and the MittagLeffler function, which are heavily used in the fractional-order calculus. 


\subsubsection{The Gamma Function}

The gamma function is defined for positive $q$ by [65] as

$$
\Gamma(q)=\int_{0}^{\infty} t^{q-1} e^{-t} d t
$$

Note that for $q \in \mathbb{N}, \Gamma(q+1)=q$ !. A more comprehensive definition is given by

$$
\Gamma(q)=\lim _{n \rightarrow \infty}\left(\frac{(n+1)^{q}}{q\left(1+\frac{q}{1}\right)\left(1+\frac{q}{2}\right) \cdots\left(1+\frac{q}{n-1}\right)\left(1+\frac{q}{n}\right)}\right) .
$$

Either definition can apply for $q \in \mathbb{C}$. The gamma function is the only analytic continuation of the factorial function to the complex plane [19].

Based on the definition of the gamma function given in Equation 3.1, the lower and upper incomplete gamma functions, $\gamma(q, x)$ and $\Gamma(q, x)$, respectively, can be defined by

$$
\gamma(q, x)=\int_{0}^{x} t^{q-1} e^{-t} d t
$$

and

$$
\Gamma(q, x)=\int_{x}^{\infty} t^{q-1} e^{-t} d t
$$

The complete gamma function plays a key role in the definitions of fractionalorder differintegral operators. The lower incomplete gamma function appears in the impulse response of systems of the form $G(s)=\frac{1}{s^{\frac{1}{n}}-a}$. 


\subsubsection{The Mittag-Leffler Function}

The Mittag-Leffler function is the entire function defined [72] by the series

$$
\mathcal{E}_{\alpha}(z)=\sum_{k=0}^{\infty} \frac{z^{k}}{\Gamma(\alpha k+1)}
$$

If $\alpha=1$, then

$$
\mathcal{E}_{\alpha}(z)=\sum_{k=0}^{\infty} \frac{z^{k}}{k !}=e^{z} .
$$

A more generalized Mittag-Leffler function can be defined by

$$
\mathcal{E}_{\alpha, \beta}(z)=\sum_{k=0}^{\infty} \frac{z^{k}}{\Gamma(\alpha k+\beta)}
$$

The Laplace transform of a specially time-weighted Mittag-Leffler function is

given by

$$
\mathcal{L}\left\{t^{\beta-1} \mathcal{E}_{\alpha, \beta}\left(a t^{\alpha}\right)\right\}=\frac{1}{s^{\beta}\left(1-a s^{-\alpha}\right)}
$$

This Laplace transform pair will be used in one formulation of the impulse response of a simple fractional-order system.

\subsection{Definitions}

The definitions of the fractional-order calculus typically involve taking a definition in the ordinary calculus and generalizing it to non-integer order. 


\subsubsection{The Grünwald-Leitnikov Definition}

The Grünwald-Leitnikov definition generalizes the iterated derivative given in Equation 2.2 by replacing $n$ with any $q \in \mathbb{C}$ and $n$ ! by $\Gamma(q+1)$, giving

$$
{ }_{a} d_{t}^{q} f(t)=\lim _{\Delta t \rightarrow 0}(\Delta t)^{-1} \sum_{k=0}^{\infty}(-1)^{k} \frac{\Gamma(q+1)}{\Gamma(k+1) \Gamma(q-k+1)} f(t-k \Delta t) .
$$

Note that $a$ is not yet needed. An equivalent definition [66] is

$$
{ }_{a} d_{t}^{q} f(t)=\lim _{N \rightarrow \infty} \sum_{k=1}^{N} \frac{\Gamma(k-q)}{\Gamma(k+1)} f\left(t-k\left(\frac{t-a}{N}\right)\right) .
$$

Note that this definition uses $a$. The Grünwald-Leitnikov definition is best suited for discrete-time simulation of fractional-order systems and implementation of fractionalorder system using digital signal processors and field programmable gate arrays [18], [41], [40].

\subsubsection{The Riemann-Liouville Definition}

The Riemann definition arises from generalizing Cauchy's formula for iterated integrals, Equation 2.6. The result is, for $q<0$

$$
{ }_{a} d_{t}^{q} f(t)=\frac{1}{\Gamma(-q)} \int_{a}^{t}(t-\tau)^{-q-1} f(\tau) d \tau
$$

The same expression holds for $q \in \mathbb{C}^{-}$. The integral does not converge if $\operatorname{Re}(q) \geq 0$. The first extension to $\operatorname{Re}(q) \geq 0$ is the Riemann-Liouville definition of the fractional 
differintegral. Choose $r<0$ and $n \in \mathbb{N}$ such that $q=r+n$. Then, due to composition of differintegrals,

$$
\begin{aligned}
{ }_{a} d_{t}^{q} f(t) & ={ }_{a} d_{t}^{r+n} f(t)={ }_{a} d_{t}^{n}{ }_{a} d_{t}^{r} f(t) \\
& =\frac{d^{n}}{d t^{n}} \frac{1}{\Gamma(-r)} \int_{a}^{t}(t-\tau)^{-r-1} f(\tau) d \tau
\end{aligned}
$$

This is the same as taking the $\mathrm{n}^{\text {th }}$ derivative of the $(q-n)^{t h}$ fractional integral of a function.

\subsubsection{The Caputo Derivative}

The Caputo derivative is a generalization of Equation 3.10 defined by

$$
{ }_{a}^{c} d_{t}^{q} f(t)=\frac{1}{\Gamma(-r)} \int_{a}^{t}(t-\tau)^{-r-1} \frac{d^{n}}{d \tau^{n}} f(\tau) d \tau
$$

This is equivalent to taking the $(q-n)^{\text {th }}$ fractional integral of the $\mathrm{n}^{\text {th }}$ derivative of a function. This is not the same as the Riemann-Liouville definition given in Equation 3.11. Consider, for example, the semiderivative of the unit step function, $\mathcal{U}_{s}(t)$ for $a>0$. In this case ${ }_{a}^{c} d_{t}^{q} \mathcal{U}_{s}(t)=0 \neq \frac{t^{-q-1}}{\Gamma(-q)}={ }_{a} d_{t}^{q} \mathcal{U}_{s}(t)$

\subsubsection{Comparison of the Definitions}

With some restrictions of functions, the Riemann-Liouville and Grünwald-Leitnikov derivatives are equivalent [77]. Let $f(t)$ be $(n-1)$-times continuously differentiable in the interval $[a, T]$ with $f^{(n)}(t)$ integrable over $[a, T]$. Then for every $p$ such that 
$0<p<n$, the Riemann-Liouville derivative exists and coincides with the GrünwaldLeitnikov derivative.

Similarly, if the lower limit of the interval on which the derivatives are acting is $-\infty$, and $f(t)$ and its derivatives behave reasonably as $t \rightarrow-\infty$, then the RiemannLiouville and Caputo derivatives are equivalent [77].

3.3 The Laplace Transform of Fractional-Order Differintegrals

Since the Laplace transform is utilized heavily in system analysis and control, the Laplace transform of the $q^{\text {th }}$-order differintegral is required. Since $\mathcal{L}\left\{{ }_{-\infty} d_{t}^{n} f(t)\right\}=$ $s^{n} \mathcal{L}\{f(t)\}$ for $n \in \mathbb{Z}$, it would be convenient if $\mathcal{L}\left\{{ }_{-\infty} d_{t}^{q} f(t)\right\}=s^{q} \mathcal{L}\{f(t)\}$ for $q \in \mathbb{C}$. Fortunately, this is the case.

Theorem 3.3.1 Let $f(s)=\mathcal{L}\{f(t)\}$, then the Laplace transform of a $q^{\text {th }}$-order derivative is given by

$$
\mathcal{L}\left\{{ }_{-\infty} d_{t}^{q} f(t)\right\}=s^{q} F(s)
$$

Proof See [77]. 


\subsection{Fractional-Order Systems}

We now consider differential equations, transfer functions, and convolution operators for fractional-order systems.

\subsubsection{Fractional-Order Differential Equations}

Definition A fractional-order differential equation is a relation,

$$
\sum_{k=1}^{n} a_{k-\infty} d_{t}^{q_{k}} x(t)=\sum_{k=1}^{m} b_{k-\infty} d_{t}^{r_{k}} u(t),
$$

where $\left\{q_{n}\right\}_{n=1}^{N} \subset \mathbb{C}$ and $\left\{r_{n}\right\}_{n=1}^{M} \subset \mathbb{C}$. If there exist $\left\{\xi_{n}\right\}_{n=1}^{N} \subset \mathbb{N}$ and $\left\{\rho_{n}\right\}_{n=1}^{M} \subset \mathbb{N}$ and $\nu \in \mathbb{C}$ such that $q_{n}=\xi_{n} \nu$ for all $1 \leq n \leq N$ and $r_{n}=\rho_{n} \nu$ for all $1 \leq n \leq M$, then the differential equation is said to be of commensurate order.

$$
\text { Note that any differential equation with }\left(\left\{q_{n}\right\}_{n=1}^{N} \cup\left\{r_{n}\right\}_{n=1}^{M}\right) \subset \mathbb{Q} \text { is }
$$
commensurate-order.

Definition The fundamental fractional-order differential equation is the differential equation given by

$$
{ }_{-\infty} d_{t}^{q} x(t)-a x(t)=u(t)
$$

\subsubsection{Fractional-Order Transfer Functions}

As with integer-order differential equations, a fractional-order differential equation may be represented as a transfer function. A system whose differential equation is 
given by Equation 3.14 can be expressed by the transfer function

$$
G(s)=\frac{\sum_{k=1}^{m} b_{k} s^{r_{k}}}{\sum_{k=1}^{n} a_{k} s^{q_{k}}}
$$

If the differential equation for a system is commensurate-order, then the transfer function is said to be of commensurate order. A commensurate-order transfer function has a partial-fraction-like expansion [9].

We note that, in general, fractional-order transfer functions are multi-valued functions. Although the value of $s$ is periodic in $\arg (s)$ with period $2 \pi$, the value of $G(s)$ is either periodic in $\arg (s)$ with period greater than $2 \pi$ or aperiodic in $\arg (s)$. As an example consider $G(s)=\frac{1}{\sqrt{s}+2} \cdot 1=e^{i 0}=e^{i 2 \pi}$, but

$$
G\left(e^{i 0}\right)=\frac{1}{\sqrt{e^{i 0}}+2}=\frac{1}{1+2}=\frac{1}{3} \neq \frac{1}{1}=\frac{1}{-1+2}=\frac{1}{\sqrt{e^{i 2 \pi}}+2}=G\left(e^{i 2 \pi}\right) .
$$

Note that $G(s)$ has a pole at $s=4 e^{i 2 \pi}$, but that $G(s)$ is analytic at $s=4 e^{i 0}$. For this reason, we make the following definitions.

Definition A Riemann sheet is the set given by $\{s \in \mathbb{C}:(2 n-1) \pi<\arg s<(2 n+1) \pi$ for some $n \in \mathbb{Z}\}$. The set $\{s \in \mathbb{C}:-\pi<\arg s<\pi\}$ is called the primary Riemann sheet.

Note that any mapping of the form $s \mapsto \frac{a}{s^{q}-a}$ is single-valued on any Riemann sheet. A fractional-order system is stable and causal if and only if its transfer function 
is analytic in the right-half plane of the primary Riemann sheet [56]. We will use this fact in our analysis of complex-order systems.

Again, as with integer-order systems, the output of a system may be written as $X(s)=G(s) U(s)$ for a transfer function $G(s)$ and an input $U(s)$. Hence, the operator representing the system, $x(t)=(\mathcal{G} u)(t)$, is the convolution operator given by

$$
x(t)=(\mathcal{G} u)(t)=\int_{-\infty}^{\infty} \tilde{g}(t-\tau) u(\tau) d \tau,
$$

where $\tilde{g}(t)$ is the impulse response of the system. Thus we need the impulse response of fractional-order operators.

\subsubsection{Fractional-Order Impulse Responses}

We now discuss three definitions of the fractional-order impulse response that are based on different functions.

The $R$ - and F-Function Representations

Let $q \in \mathbb{C}$. The $F$-function is defined as the impulse response for the fundamental fractional-order differential equation given in Equation 3.15 [31]. It is obtained by performing long-division on the transfer function of the fundamental fractional-order system. Assuming $\left|\frac{a}{s^{q}}\right|<1$, we can write

$$
\begin{aligned}
\frac{1}{s^{q}-a} & =\frac{1}{s^{q}}+\frac{a}{s^{2 q}}+\frac{a^{2}}{s^{3 q}}+\frac{a^{3}}{s^{4 q}}+\cdots \\
& =\sum_{k=0}^{\infty} \frac{a^{k}}{s^{(k+1) q}} .
\end{aligned}
$$


Using the Laplace transform pair from [61] given by

$$
\mathcal{L}\left\{\frac{t^{q}}{\Gamma(q+1)}\right\}=\frac{1}{s^{q+1}},
$$

the inverse Laplace transform of the series is taken term by term to give

$$
\mathcal{L}^{-1}\left\{\frac{1}{s^{q}-a}\right\}=F_{q}(a, t)=\sum_{k=0}^{\infty} \frac{a^{k} t^{(k+1) q-1}}{\Gamma((k+1) q)}
$$

Similarly, for $\nu \in \mathbb{C}$, the $R$-Function is defined as the impulse response for the differential equation

$$
{ }_{-\infty} d_{t}^{q} x(t)-a x(t)={ }_{-\infty} d_{t}^{\nu} u(t)
$$

Again, using long division, and assuming $\left|\frac{a}{s^{q}}\right|<1$,

$$
\begin{aligned}
\frac{s^{\nu}}{s^{q}-a} & =\frac{1}{s^{q-\nu}}+\frac{a}{s^{2 q-\nu}}+\frac{a^{2}}{s^{3 q-\nu}}+\frac{a^{3}}{s^{4 q-\nu}}+\cdots \\
& =\sum_{k=0}^{\infty} \frac{a^{k}}{s^{(k+1) q-\nu}} .
\end{aligned}
$$

Using the Laplace transform pair given by Equation 3.19, the inverse Laplace transform of the series is taken term by term to give

$$
R_{q, \nu}(a, t)=\sum_{k=0}^{\infty} \frac{a^{k} t^{(k+1) q-1-\nu}}{\Gamma((k+1) q-\nu)}
$$


Hence the kernels of the convolution operator representations for the systems whose transfer functions are $\frac{1}{s^{q}-a}$ and $\frac{s^{\nu}}{s^{q}-a}$ are

$$
\tilde{g}(t-\tau)=F_{q}(a, t-\tau)
$$

and

$$
\tilde{g}(t-\tau)=R_{q, \nu}(a, t-\tau),
$$

respectively.

The Mittag-Leffler Function Representation

Recall that the generalized Mittag-Leffler function, $\mathcal{E}_{\alpha, \beta},(t)$ is involved in the Laplacetransform pair,

$$
\mathcal{L}\left\{t^{\beta-1} \mathcal{E}_{\alpha, \beta}\left(a t^{\alpha}\right)\right\}=\frac{1}{s^{\beta}\left(1-a s^{-\alpha}\right)}
$$

as given in 3.7. Then the Laplace transform for the function, $t^{q-1} \mathcal{E}_{q, q}\left(a t^{q}\right)$ is given by

$$
\mathcal{L}\left\{t^{q-1} \mathcal{E}_{q, q}\left(a t^{q}\right)\right\}=\frac{1}{s^{q}\left(1-a s^{-q}\right)}=\frac{1}{s^{q}-a},
$$

which indicates that this function using the Mittag-Leffler function is the impulse response for the fundamental fractional-order differential equation. Although in the literature this function is called a Mittag-Leffler function, it must be noted that a factor of $t^{q-1}$ is included, so the impulse response is actually a time-weighted MittagLeffler function. 
Thus, the kernel of the integral operator representation for the system whose transfer function is $\frac{1}{s^{q}-a}$ is

$$
\tilde{g}(t-\tau)=(t-\tau)^{q-1} \mathcal{E}_{q, q}\left(a(t-\tau)^{q}\right)
$$

When more than one fractional-order derivative is present in the system, the impulse response is more complicated. [77] gives impulse responses for fractional-order differential equations with one, two, three, and four fractional-order differintegrals.

The Representation of Miller and Ross

Miller and Ross [58] first define a function, $E_{t}(q, a)$ by

$$
E_{t}(q, a)=\frac{e^{a t}}{a^{q} \Gamma(q)} \gamma(q, a t)
$$

This function can be rewritten as

$$
\begin{aligned}
E_{t}(q, a) & =\frac{e^{a t}}{a^{q} \Gamma(q)} \int_{0}^{a t} \xi^{q-1} e^{-\xi} d \xi \\
& =\frac{1}{a^{q}} \int_{0}^{a t} \frac{\xi^{q-1}}{\Gamma(q)} e^{a t-\xi} d \xi \\
& =\int_{0}^{t} e^{a(t-\tau)} \frac{\tau^{q-1}}{\Gamma(q)} d \tau \\
& =e^{a t} * \frac{t^{q-1}}{\Gamma(q)}
\end{aligned}
$$


Hence, it can be seen that

$$
\mathcal{L}\left\{E_{t}(q, a)\right\}=\mathcal{L}\left\{e^{a t}\right\} \mathcal{L}\left\{\frac{t^{q-1}}{\Gamma(q)}\right\}=\frac{s^{-q}}{s-a} .
$$

For $n \in \mathbb{N}$, it is an algebra fact that

$$
x^{n}-a^{n}=(x-a)\left(\sum_{k=1}^{n} a^{k-1} x^{n-k}\right) .
$$

Subsequently, for $n \in \mathbb{N}$ with $x=s^{\frac{1}{n}}$,

$$
s-a^{n}=\left(s^{\frac{1}{n}}-a\right)\left(\sum_{k=1}^{n} a^{k-1} s^{1-\frac{k}{n}}\right)
$$

or

$$
\frac{1}{s^{\frac{1}{n}}-a}=\frac{\sum_{k=1}^{n} a^{k-1} s^{1-\frac{k}{n}}}{s-a^{n}}=\sum_{k=1}^{n} a^{k-1} \frac{s^{1-\frac{k}{n}}}{s-a^{n}} .
$$

The inverse Laplace transform of $\frac{1}{s^{\frac{1}{n}}-a}$ is thus,

$$
\begin{aligned}
\mathcal{L}^{-1}\left\{\frac{1}{s^{\frac{1}{n}}-a}\right\} & =\mathcal{L}^{-1}\left\{\sum_{k=1}^{n} a^{k-1} \frac{s^{1-\frac{k}{n}}}{s-a^{n}}\right\} \\
& =\sum_{k=1}^{n} a^{k-1} \mathcal{L}^{-1}\left\{\frac{s^{1-\frac{k}{n}}}{s-a^{n}}\right\} \\
& =\sum_{k=1}^{n} a^{k-1} E_{t}\left(\frac{k}{n}-1, a^{n}\right) .
\end{aligned}
$$


Hence, the kernel of the integral operator for the system whose transfer function is $\frac{1}{s^{\frac{1}{n}}-a}$ is

$$
\tilde{g}(t-\tau)=\sum_{k=1}^{n} a^{k-1} E_{t-\tau}\left(\frac{k}{n}-1, a^{n}\right)
$$

This representation is advantageous because it involves a finite sum rather than an infinite sum. Thus, the sum and integral sign of the integral operator are interchangeable, without having to resort to convergence theorems for integrals. This representation has a disadvantage in that only rational-order systems can be studied.

Equivalence of Convolution Kernels

The three convolution kernels presented above are formed using differently defined functions. We now show, via direct comparison in the time-domain, that the functions are the same for $q=\frac{1}{n}$ for $n \in \mathbb{N}$.

Theorem 3.4.1 Let $q \in \mathbb{C}$. Then $F_{q}(a, t)=t^{q-1} \mathcal{E}_{q, q}\left(a t^{q}\right)$.

Proof Note that using the definition of the generalized Mittag-Leffler function, in Equation 3.6,

$$
\begin{aligned}
t^{q-1} \mathcal{E}_{q, q}\left(a t^{q}\right) & =t^{q-1} \sum_{k=0}^{\infty} \frac{\left(a t^{q}\right)^{k}}{\Gamma(q k+q)} \\
& =\sum_{k=0}^{\infty} \frac{a^{k} t^{(k+1) q-1}}{\Gamma((k+1) q)} \\
& =F_{q}(a, t)
\end{aligned}
$$


by the definition of the $F$-function in Equation 3.20. Thus, the convolution kernels are equal.

Theorem 3.4.2 Let $n \in \mathbb{N}$ and let $q=\frac{1}{n}$. Then the equality,

$$
F_{q}(a, t)=t^{q-1} \mathcal{E}_{q, q}\left(a t^{q}\right)=\sum_{k=1}^{n} a^{k-1} E_{t}\left(\frac{k}{n}-1, a^{n}\right)
$$

holds.

Proof Because the previous theorem establishes the equality of the left and middle terms in Equation 3.30, all that remains is to demonstrate that the middle term is equal to the right term. Consider

$$
\begin{aligned}
\sum_{k=1}^{n} a^{k-1} E_{t}\left(\frac{k}{n}-1, a^{n}\right) & =\sum_{j=1}^{n} a^{j-1} t^{\frac{j}{n}-1} \sum_{k=0}^{\infty} \frac{\left(a^{n} t\right)^{k}}{\Gamma\left(\frac{j}{n}-1+k+1\right)} \\
& =\sum_{j=1}^{n} \sum_{k=0}^{\infty} \frac{a^{n k+j-1} t^{\frac{1}{n}+k-1}}{\Gamma\left(\frac{j}{n}+k\right)} \\
& =\sum_{k=0}^{\infty} \sum_{j=1}^{n} \frac{a^{n k+j-1} t^{\frac{n k+j}{n}-1}}{\Gamma\left(\frac{n k+j}{n}\right)} \\
& =\sum_{\ell=0}^{\infty} \frac{a^{\ell} t^{\ell+1}-1}{\Gamma\left(\frac{\ell+1}{n}\right)}=t^{\frac{1}{n}-1} \mathcal{E}_{\frac{1}{n}, \frac{1}{n}}\left(a t^{\frac{1}{n}}\right) .
\end{aligned}
$$

Hence, the equality holds, and the three functions are the same for $q=\frac{1}{n}$. 
These impulse responses will be used to consider the Hankel operator of a fractional-order system in Chapter 7.

\subsection{Approximation of Fractional-Order Operators}

One method for approximating fractional-order operators is accomplished in the frequency domain. This method was developed by Oustaloup [71] and Chareff et al. [16]. Rational approximations are made for operators of the type $s^{-q}$ that are within

a certain error bound of the operator's Bode magnitude plot over a defined frequency range. Note that the Bode magnitude plot for $s^{-q}$ is a line with a slope of $-20 q \frac{\mathrm{dB}}{\mathrm{dec}}$ that is $0 \mathrm{~dB}$ at 1rad. This approximation method may be accomplished as follows.

First a frequency range, $\omega_{a} \leq \omega \leq \omega_{b}$, and error bound, $M_{e}$, are selected. The pole-to-zero ratio, $a$, and the zero-to-pole ratio, $b$, are defined by

$$
a=10^{\frac{M_{e}}{10(1-q)}}
$$

and

$$
b=10^{\frac{M_{e}}{10 q}},
$$

respectively. The number of poles required for the approximation is determined by

$$
N-1<\frac{\log \left(\frac{\omega_{b}}{\omega_{a}}\right)}{\log (a b)} \leq N
$$


The first pole, $p_{0}$, is placed at $p_{0}=\omega_{a} 10^{\frac{M_{e}}{20 q}}$. Starting at $k=0$, poles are placed at

$$
p_{k}=(a b)^{k} \omega_{a}
$$

and, starting at $k=0$, zeroes are placed at

$$
z_{k}=a^{k+1} b^{k} \omega_{a}
$$

Although the slope of the magnitude response is correct, the gain of the approximation is not, so a gain applied to the approximation to match that of the integrator is applied to the system. Table 3.1 gives approximations with $2.5 \mathrm{~dB}$-error over $\omega \in[0.01,100]$ for $s^{-\frac{k}{10}}$ for $1 \leq k \leq 9$. Hartley and Lorenzo give a similar table for approximations with 2dB-error in [34].

Once the Hankel singular values for a fractional-order system are determined, it will be possible to evaluate how close to the optimal $n$-dimensional approximation an approximation made using the technique of Oustaloup and Chareff et al. is. Although it is beyond the scope of this dissertation, if the Schmidt pairs could be determined, then it would be possible to construct the optimal $n$-dimensional approximation to a fractional-order system. 
Table 3.1: 2.5dB-Approximations for $s^{\frac{-k}{10}}$ over $\omega \in[0.01,100]$ for $1 \leq k \leq 9$.

\begin{tabular}{cc}
\hline $\begin{array}{c}\text { Fractional-Order } \\
\text { Operator }\end{array}$ & Approximation \\
\hline$s^{-\frac{1}{10}}$ & $\frac{2016 s^{2}+22950 s+434.3}{s^{3}+3600 s^{2}+21580 s+215.4}$ \\
$s^{-\frac{1}{5}}$ & $\frac{190.3 s^{3}+5357 s^{2}+4017 s+80.24}{s^{4}+500.7 s^{3}+6682 s^{2}+2438 s+23.71}$ \\
$s^{-\frac{3}{10}}$ & $\frac{114.5 s^{4}+10380 s^{3}+57030 s^{2}+20050 s+426.8}{s^{5}+617.8 s^{4}+23130 s^{3}+55600 s^{2}+8585 s+80.31}$ \\
$s^{-\frac{2}{5}}$ & $\frac{26.68 s^{4}+1021 s^{3}+3255 s^{2}+928 s+22.02}{s^{5}+161.4 s^{5}+2171 s^{3}+3255 s^{2}+928 s+2.61}$ \\
$s^{-\frac{1}{2}}$ & $\frac{13.39 s^{4}+470.4 s^{3}+1501 s^{2}+470.4 s+13.39}{s^{5}+111.1 s^{4}+1122 s^{3}+1122 s^{2}+111.1 s+1}$ \\
$s^{-\frac{3}{5}}$ & $\frac{3.813 s^{3}+21.41 s^{2}+9.938 s+0.3813}{s^{4}+14.67 s^{3}+17.9 s^{2}+1.956 s+0.01778}$ \\
$s^{-\frac{7}{10}}$ & $\frac{3.982 s^{3}+69.7 s^{2}+73.63 s+4.694}{s^{4}+39.85 s^{3}+96.17 s^{2}+14.85 s+0.1389}$ \\
$s^{-\frac{4}{5}}$ & $\frac{2.239 s^{2}+14.94 s+2.586}{s^{3}+13.71 s^{2}+5.007 s+0.0487}$ \\
$s^{-\frac{9}{10}}$ & $\frac{1.697 s+5.367}{s^{2}+6.005 s+0.05995}$
\end{tabular}

\subsection{Initialization of Fractional-Order Systems}

Often in real applications, the analysis of a system starts at some initial time $t_{0}$ with knowledge of the initial state. The initial state contains all of the effect of the past history of the system. For a $k^{\text {th }}$-order system an initial state that is $k$-dimensional is required to properly account for the effect of the past.

Because fractional-order systems are infinite-dimensional, they require initializations more complicated than those for integer-order systems. Although it is commonly held that the Caputo derivative properly accounts for initial conditions using a finite number of initial conditions [15], it has been shown that in general, the Caputo initialization cannot account for all types of initialization $[2,3]$. The Caputo derivative properly accounts for initial conditions if the system has been held at that initial condition for all time $[49,50,52]$. As such, to account for an arbitrary 
initialization, an infinite amount of information is necessary [46, 47, 48, 32, 33, 68]. Using the Riemann-Liouville definition, Lorenzo and Hartley derive an initialization function $\Psi(f, q,-\infty, 0, t)$ as follows.

Without a loss of generality, it can be assumed that the initialization period is $t \in \mathbb{R}_{-}$, and that the present problem occurs for $t \in \mathbb{R}_{+}$. Define the initialization function $\Psi(f, q,-\infty, 0, t)$ by

$$
\Psi(f, q,-\infty, 0, t)=\frac{d^{n}}{d t^{n}} \frac{1}{\Gamma(-(q-n))} \int_{-\infty}^{0}(t-\tau)^{-(q-n)-1} f(\tau) d \tau
$$

Recall the definition of the Riemann-Liouville definition of the differintegral

$$
{ }_{-\infty} d_{t}^{q} f(t)=\frac{d^{n}}{d t^{n}} \frac{1}{\Gamma(-r)} \int_{-\infty}^{t}(t-\tau)^{-r-1} f(\tau) d \tau,
$$

where $n \in \mathbb{N}, r<0$, and $q=r+n$. Then, for $t \in \mathbb{R}_{+}$,

$$
\begin{aligned}
{ }_{-\infty} d_{t}^{q} f(t) & =\frac{d^{n}}{d t^{n}} \frac{1}{\Gamma(-r)} \int_{-\infty}^{0}(t-\tau)^{-r-1} f(\tau) d \tau+\frac{d^{n}}{d t^{n}} \frac{1}{\Gamma(-r)} \int_{0}^{t}(t-\tau)^{-r-1} f(\tau) d \tau \\
& =\frac{d^{n}}{d t^{n}} \frac{1}{\Gamma(-r)} \int_{-\infty}^{0}(t-\tau)^{-r-1} f(\tau) d \tau+{ }_{0} d_{t}^{q} f(t) \\
& =\Psi(f, q,-\infty, 0, t)+{ }_{0} d_{t}^{q} f(t) \\
& ={ }_{0} d_{t}^{q} f(t)+\Psi(f, q,-\infty, 0, t) .
\end{aligned}
$$

$\Psi(f, q,-\infty, 0, t)$ is called the initialization- or psi-function and properly takes into account the initialization of a single differintegral [47]. 
In general, $\Psi(f, q,-\infty, 0, t)$ is difficult to use $[52,51,53]$. As an example, consider the system whose differential equation is

$$
{ }_{-\infty} d_{t}^{q} x(t)+\alpha x(t)=\beta u(t)
$$

with an initial time of $t=0$. That is, we wish to analyze the system whose differential equation is

$$
{ }_{0} d_{t}^{q} x(t)+\alpha x(t)=\beta u(t)
$$

However, there is a loss of information changing the differential equation from Equation 3.32 to Equation 3.33. We must modify Equation 3.33 to become

$$
{ }_{0} d_{t}^{q} x(t)+\Psi(x, q,-\infty, 0, t)+\alpha x(t)=\beta u(t) .
$$

We will make the substitution

$$
\xi(t)=x(t)+{ }_{0} d_{t}^{-q} \Psi(x, q,-\infty, 0, t)
$$

Equation 3.34 becomes

$$
{ }_{0} d_{t}^{q} \xi(t)+\alpha \xi(t)=\beta u(t)+\alpha{ }_{0} d_{t}^{-q} \Psi(x, q,-\infty, 0, t)
$$


which we can write as the convolution operator

$$
\xi(t)=\int_{0}^{t} F_{q}(\alpha, t-\tau)\left(\beta u(\tau)+\alpha_{0} d_{\tau}^{-q} \Psi(x, q,-\infty, 0, \tau)\right) d \tau
$$

Thus, the solution to Equation 3.33 properly accounting for initialization is

$$
\begin{aligned}
x(t)= & \int_{0}^{t} F_{q}(\alpha, t-\tau)\left(\beta u(\tau)+\alpha_{0} d_{\tau}^{-q} \Psi(x, q,-\infty, 0, \tau)\right) d \tau \\
& -{ }_{0} d_{t}^{-q} \Psi(x, q,-\infty, 0, t) .
\end{aligned}
$$

As evidenced by Equation 3.35, the $(-q)^{\text {th }}$ derivative of the psi-function can be considered a perturbation to the associated state [46].

The use of the Hankel operator also properly accounts for the initialization of a system. The Hankel operator accomplishes this for the fractional-order system as a whole, rather than on each individual fractional-order derivative like the psifunction. However, both approaches do properly account for the past behavior of the fractional-order system in the future response.

\section{$3.7 \quad$ Examples}

We now consider two systems that include the semi-infinite line. Because of these inclusions, the systems are of fractional order. 


\subsubsection{Resistor-Terminated Semi-Infinite Line Example}

Consider a resistor-terminated semi-infinite line, whose circuit diagram is given in

Figure 3.1. This system has a transfer function $G_{R}(s)$ given by

$$
\begin{aligned}
G_{R}(s) & =\frac{V_{o}(s)}{V_{i}(s)}=\frac{\frac{A}{s^{\frac{1}{2}}}}{R+\frac{A}{s^{\frac{1}{2}}}} \\
& =\frac{\frac{A}{R}}{s^{\frac{1}{2}}+\frac{A}{R}} .
\end{aligned}
$$

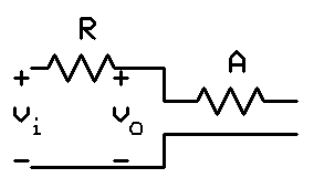

Figure 3.1: Resistor-Terminated Semi-Infinite Line.

The differential equation associated with this system is

$$
{ }_{-\infty} d_{t}^{\frac{1}{2}} x(t)+\frac{A}{R} x(t)=\frac{A}{R} f(t) .
$$

The impulse response $\tilde{g}_{R}(t)$ can be written using either the $F$-function approach as

$$
\tilde{g}_{R}(t)=\frac{A}{R} F_{\frac{1}{2}}\left(-\frac{A}{R}, t\right)=\frac{A}{R} \sum_{k=0}^{\infty} \frac{\left(-\frac{A}{R}\right)^{k} t^{\frac{k-1}{2}}}{\Gamma\left(\frac{k+1}{2}\right)}
$$


or using the approach of Miller and Ross [58] as

$$
\tilde{g}_{R}(t)=\frac{A}{R} \sum_{k=1}^{2}\left(-\frac{A}{R}\right)^{k-1} E_{t}\left(\frac{k-2}{2},\left(-\frac{A}{R}\right)^{2}\right) .
$$

Note that $G_{R}\left(|s| e^{i \arg (s)}\right)=G_{R}\left(|s| e^{i(\arg (s)+4 \pi)}\right)$, so there are only two unique Riemann sheets. Every Riemann sheet is a copy of either the primary Riemann sheet, which is given by $\{s \in \mathbb{C}: \arg (s) \in(-\pi, \pi)\}$, or the secondary Riemann sheet, which is given by $\{s \in \mathbb{C}: \arg (s) \in(\pi, 3 \pi)\}$. Also note that $G_{R}(s)$ has a pole at $s_{p}=\left(\frac{A}{R}\right)^{2} e^{i 2 \pi}$. Because $\arg \left(s_{p}\right) \notin(-\pi, \pi)$, this pole is on the secondary Riemann sheet rather than the primary Riemann sheet. Because of this the system exhibits ultradamping; the system decays without oscillation quickly from its initial condition, but decays more slowly as time progresses [33]. Note that no matter the value of $\frac{A}{R}$, $\lim _{t \rightarrow 0^{+}} \tilde{g}_{R}(t)=\infty$. The instantaneous rate of decay at any time $t$ increases as $\frac{A}{R}$ increases. Figure 3.2 shows $\tilde{g}_{R}(t)$ for $\frac{A}{R} \in\left\{\frac{1}{2}, 1,2\right\}$. The basic shape of the impulse response is the same.

Consider the magnitude and phase plots of $G_{R}(i \omega)$. At low frequencies $\omega<<$ $\left(\frac{A}{R}\right)^{2}$, the magnitude is approximately 0dB. At high frequencies $\omega>>\left(\frac{A}{R}\right)^{2}$, the magnitude rolls off at $10 \frac{\mathrm{dB}}{\mathrm{dec}}$. The phase of $G_{R}(i \omega)$ for high frequencies is $-45^{\circ}$. For high frequencies, the phase of an integer-order system is an integer multiple of $90^{\circ}$. At high frequencies the phase difference between $G_{R}(i \omega)$ and an integer-order approximation will be at least $45^{\circ}$. Figure 3.3 shows shows the magnitude and phase plots of $G_{R}(i \omega)$ with $\frac{A}{R}=1$. Increasing $\frac{A}{R}$ increases the bandwidth of the system. 


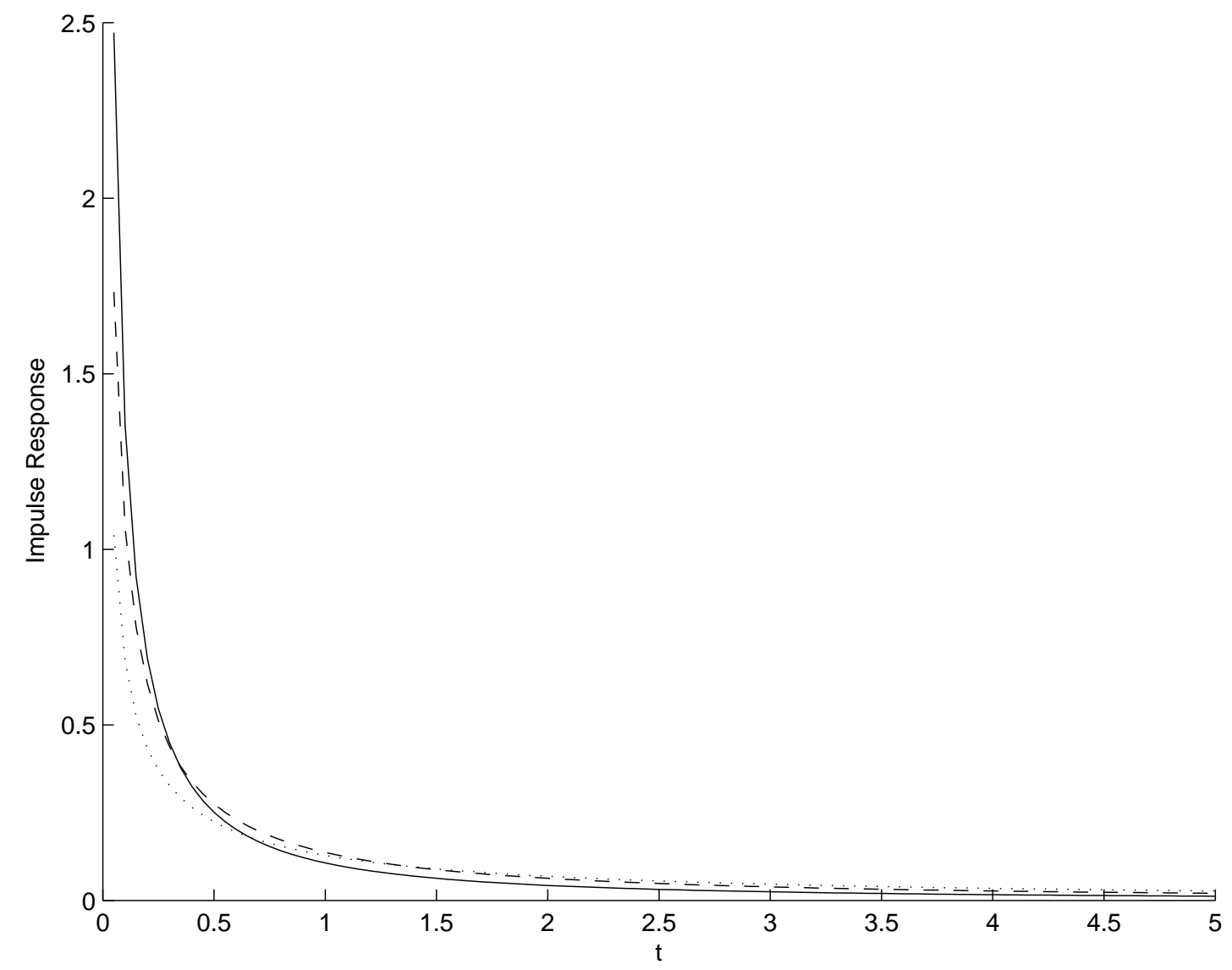

Figure 3.2: Impulse Response for Resistor-Terminated Semi-Infinite Line with $\frac{A}{R}=\frac{1}{2}$ (dotted), $\frac{A}{R}=1$ (dashed), and $\frac{A}{R}=2$ (solid).

For the remainder of this document, we will consider the case where $\frac{A}{R}=1$.

Using the approximation

$$
s^{-\frac{1}{2}}=\frac{13.39 s^{4}+470.4 s^{3}+1501 s^{2}+470.4 s+13.39}{s^{5}+111.1 s^{4}+1122 s^{3}+1122 s^{2}+111.1 s+1}
$$


from Table 3.1, we can construct an approximation to $G_{R}(s)$ as

$$
\begin{aligned}
\hat{G}_{R}(s) & =\frac{1}{\frac{s^{5}+111.1 s^{4}+1122 s^{3}+1122 s^{2}+111.1 s+1}{13.39 s^{4}+470.4 s^{3}+1501 s^{2}+440.4 s+13.39}+1} \\
& =\frac{13.39 s^{4}+470.4 s^{3}+1501 s^{2}+470.4 s+13.39}{s^{5}+124.49 s^{4}+1592.4 s^{3}+2623 s^{2}+481.5 s+14.39} .
\end{aligned}
$$
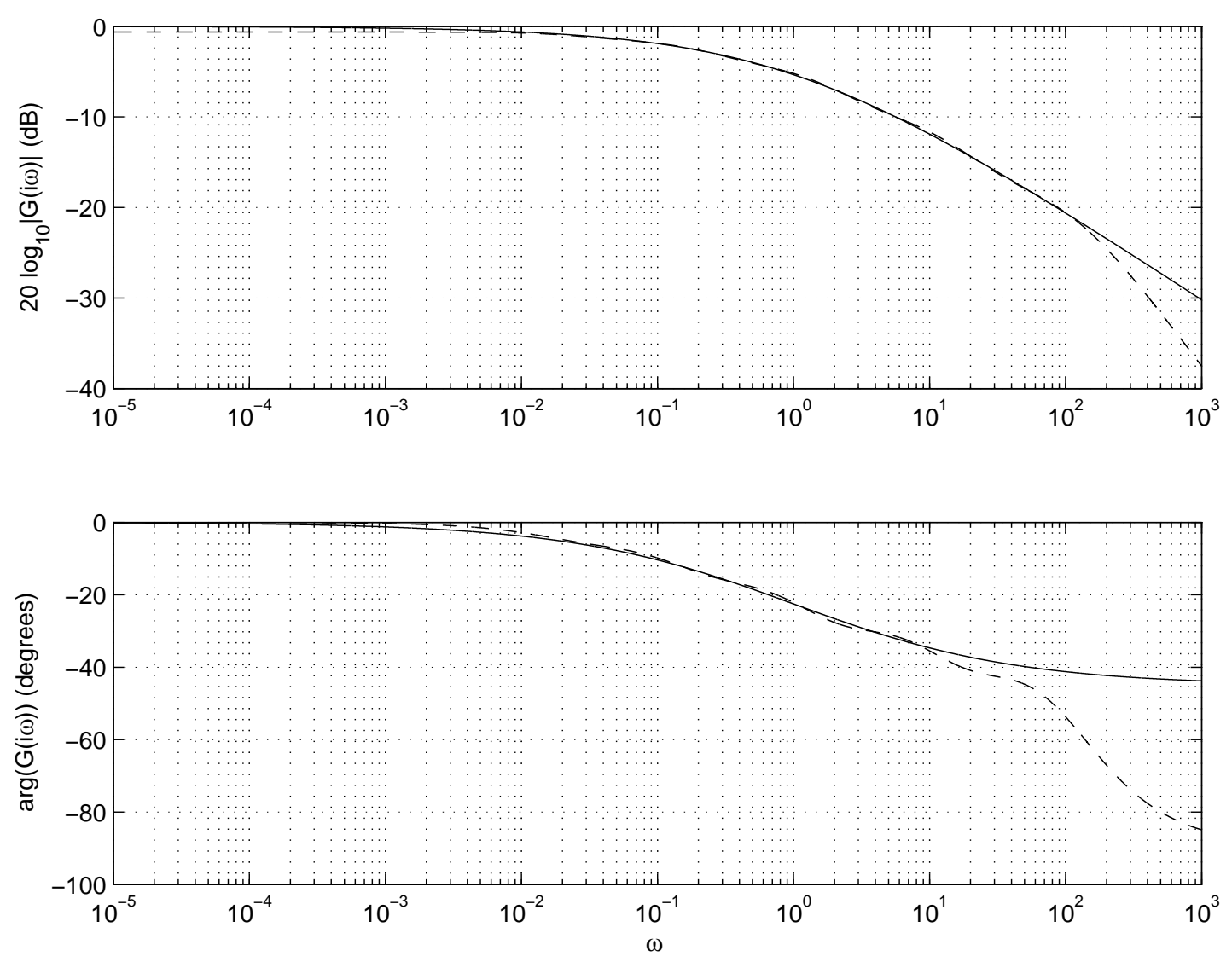

Figure 3.3: Magnitude and Phase Responses for a Resistor-Terminated Semi-Infinite Line with $\frac{A}{R}=1$ (solid) and a Fifth-Order Approximation (dashed).

The magnitude and phase plots of this fifth-order approximation are shown in Figure 3.3. The approximation is good in magnitude for $10^{-2}<\omega<10^{2}$ and good in phase for $10^{-2}<\omega<10^{1}$. 


\subsubsection{Inductor-Terminated Semi-Infinite Line}

Another example is the inductor-terminated semi-infinite line. This problem, which has its roots in transatlantic communication in the late nineteenth century, was studied by Heaviside [36] and Bush [14]. The system is shown in Figure 3.4.

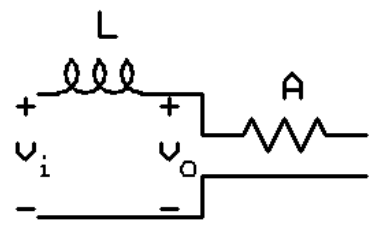

Figure 3.4: Inductor-Terminated Semi-Infinite Line.

The transfer function for this system is

$$
\begin{aligned}
G_{L}(s) & =\frac{V_{o}(s)}{V_{i}(s)}=\frac{\frac{A}{s^{\frac{1}{2}}}}{s L+\frac{A}{s^{\frac{1}{2}}}} \\
& =\frac{\frac{A}{L}}{s^{\frac{3}{2}}+\frac{A}{L}} .
\end{aligned}
$$

The differential equation for this system is

$$
{ }_{-\infty} d_{t}^{\frac{3}{2}} x(t)+\frac{A}{L} x(t)=\frac{A}{L} f(t)
$$

Using the $F$-function approach, the impulse response for this system is

$$
\tilde{g}_{L}(t)=\frac{A}{L} F_{\frac{3}{2}}\left(-\frac{A}{L}, t\right)=\frac{A}{L} \sum_{k=0}^{\infty} \frac{\left(-\frac{A}{L}\right)^{k} t^{\frac{3 k+1}{2}}}{\Gamma\left(\frac{3 k+3}{2}\right)}
$$


Note that $G_{L}\left(|s| e^{i \arg (s)}\right)=G_{L}\left(|s| e^{i(\arg (s)+4 \pi)}\right)$, so there are only two unique Riemann sheets. Also note that $G_{L}(s)$ has poles at $s_{1}=\left(\frac{A}{L}\right)^{\frac{2}{3}} e^{i 2 \pi}$ and $s_{2,3}=$ $\left(\frac{A}{L}\right)^{\frac{2}{3}} e^{ \pm i \frac{2}{3} \pi}$. Because $\arg \left(s_{1}\right) \notin(-\pi, \pi)$, this pole is on the secondary Riemann sheet rather than the primary Riemann sheet. The poles $s_{2,3}$ are in the left-half plane of the primary Riemann sheet. The contribution of $s_{1}$ to the impulse response rapidly decays without oscillation. As $\frac{A}{L}$ increases, the rate of decay increases. The contribution of $s_{2,3}$ to the impulse response is a decaying sinusoid, as would be expected from an integer-order system with complex conjugate poles in the left-half plane. The contribution from $s_{2,3}$ dominates the response. The effects on the amplitude, overshoot, settling time, and natural frequency of contribution from $s_{2,3}$ of changing $\frac{A}{L}$ are the same as changing an integer-order system with poles at $s_{2,3}$.

Figure 3.5 shows $\tilde{g}_{L}(t)$ for $\frac{A}{L} \in\left\{\frac{1}{2}, 1,2\right\}$. The basic shape of the impulse response is the same. The amplitude and speed of the impulse response increase as $\frac{A}{L}$ increases. At low frequencies $\omega<<\left(\frac{A}{L}\right)^{\frac{2}{3}}$, the magnitude is approximately 0dB. The magnitude response has a resonance at $\omega=\left(\frac{A}{L}\right)^{\frac{2}{3}}$ contributed by the poles $s_{2,3}$. At high frequencies $\omega<<\left(\frac{A}{L}\right)^{\frac{2}{3}}$, the magnitude rolls off at $30 \frac{\mathrm{dB}}{\mathrm{dec}}$. The phase of $G_{L}(i \omega)$ for high frequencies is $135^{\circ}$. For high frequencies, the phase of an integer-order system is an integer multiple of $90^{\circ}$. At high frequencies the phase difference between $G_{L}(i \omega)$ and an integer-order approximation will be at least $45^{\circ}$. Figure 3.6 shows shows the magnitude and phase plots of $G_{L}(i \omega)$ for $\frac{A}{L}=1$. 


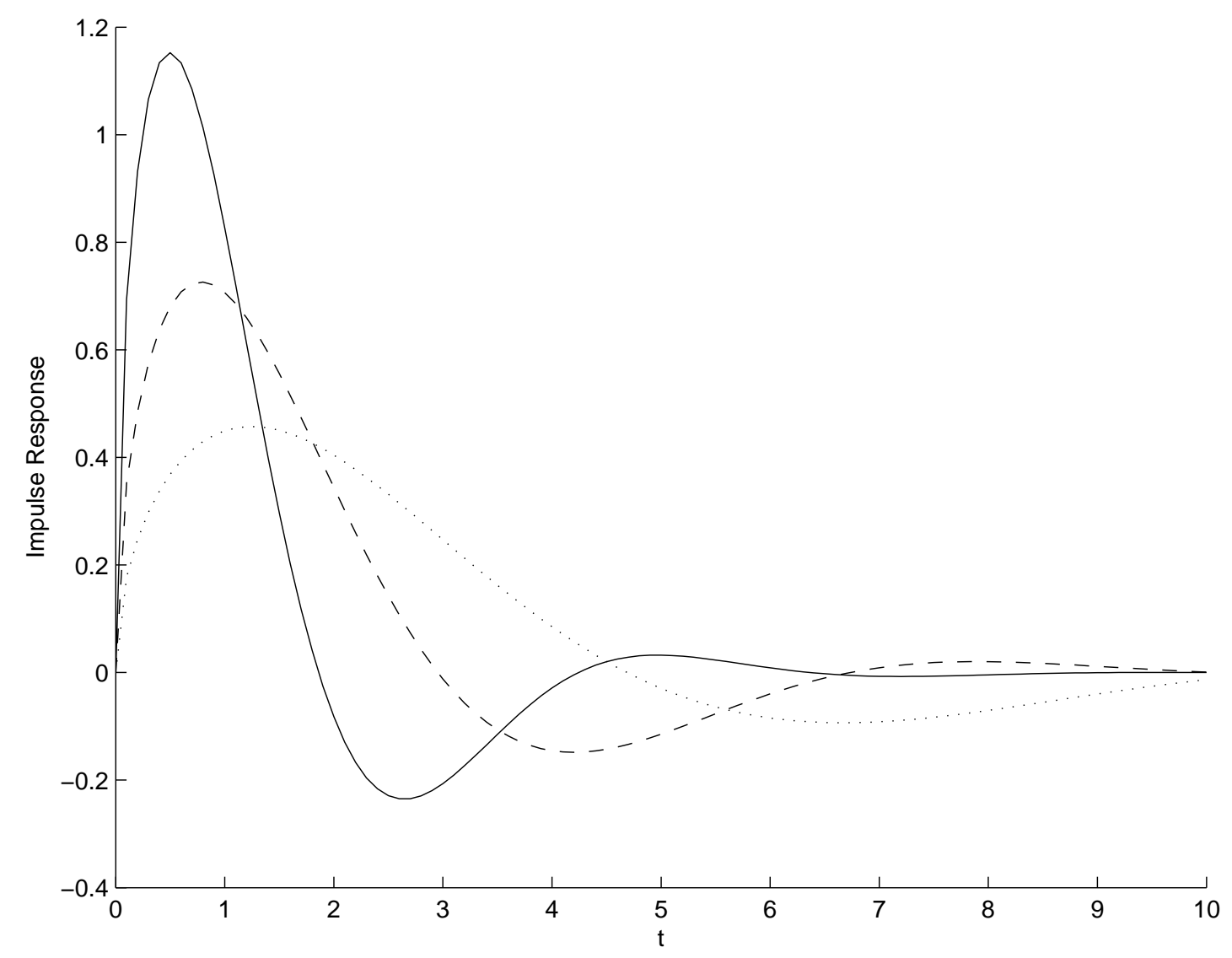

Figure 3.5: Impulse Response for Inductor-Terminated Semi-Infinite Line with $\frac{A}{L}=\frac{1}{2}$ (dotted), $\frac{A}{L}=1$ (dashed), and $\frac{A}{L}=2$ (solid).

For the remainder of this document, we will consider the case where $\frac{A}{L}=1$.

$G_{L}(s)$ can be approximated using Equation 3.40 by

$$
\begin{aligned}
\hat{G}_{L}(s) & =\frac{1}{s \frac{s^{5}+111.1 s^{4}+1122 s^{3}+1122 s^{2}+111.1 s+1}{13.39 s^{4}+470.4 s^{3}+1501 s^{2}+470.4 s+13.39}+1} \\
& =\frac{13.39 s^{4}+470.4 s^{3}+1501 s^{2}+470.4 s+13.39}{s^{6}+111.1 s^{5}+1135.39 s^{4}+1592.4 s^{3}+1612.1 s^{2}+471.4 s+13.39} .
\end{aligned}
$$


This sixth-order approximation is shown in Figure 3.6. The approximation is good in magnitude for $10^{-2} \leq \omega \leq 10^{2}$ and good in phase for $10^{-2} \leq \omega \leq 10^{1}$.
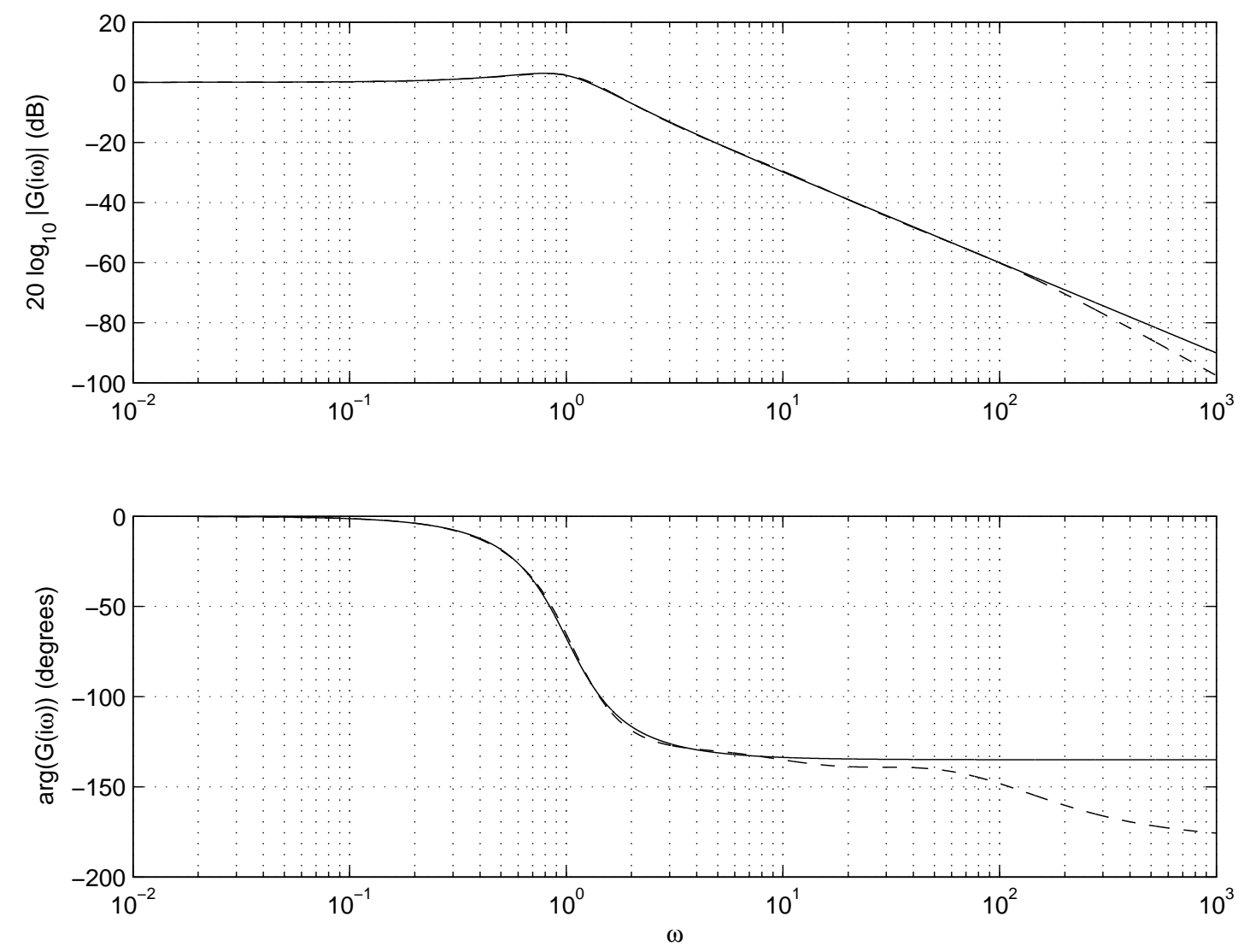

Figure 3.6: Magnitude and Phase Responses for a Resistor-Terminated Semi-Infinite Line with $\frac{A}{L}=1$ (solid) and a Fifth-Order Approximation (dashed).

\subsection{Conclusions}

In this chapter the concepts of derivatives and systems of integer order have been extended to those of non-integer order. Non-integer order differintegrals have been defined, and fractional-order systems have been represented as differential equations, transfer functions, and convolution operators. A method by which fractional-order 
systems may be approximated has been presented. We have discussed the complexity in finding an initial-condition response which properly accounts for the past behavior of the system in the future response. Finally, two examples illustrating these concepts have been considered. We now examine complex-order systems in more depth. 


\section{CHAPTER IV}

\section{CONJUGATE-ORDER OPERATORS}

The fractional-order operator has been generalized to the complex-order operator $[44,54,69]$. Conjugate-order operators are operators where both a differintegral of complex order $q \in \mathbb{C}$ and a differintegral of complex-conjugate order $\bar{q}$ are present[6]. In this chapter we consider conjugate-order differintegrals and conjugate-order sys-

tems of the form $G(s)=\frac{r}{s^{q}-a}+\frac{\bar{r}}{s^{\bar{q}}-\bar{a}}$. We show that such systems have real-valued impulse responses and, if $\operatorname{Re}(q) \neq 0$, have a finite number of poles on the primary Riemann sheet. For a fixed $a \in \mathbb{C}$ we describe the set of $q \in \mathbb{C}$ such that $G(s)$ corresponds to a stable, causal system. Finally, for a fixed $q \in \mathbb{C}$ we describe the set of $a \in \mathbb{C}$ such that $G(s)$ corresponds to a stable, causal system.

\subsection{Complex-Order Differintegrals}

In general, the complex-order differintegral acting on a function $f(t)$ is defined as

$$
{ }_{-\infty} d_{t}^{-q} f(t)={ }_{-\infty} d_{t}^{-(u+i v)} f(t)
$$


for $q=u+i v$. Let $G(s)$ be the Laplace-domain representation of this complex-order differintegral operator. $G(s)$ is given by

$$
G(s) F(s)=\mathcal{L}\left\{{ }_{-\infty} d_{t}^{-q} f(t)\right\}=s^{-(u+i v)} F(s)=s^{-u} s^{-i v} F(s)=s^{-u} e^{-i v \ln (s)} F(s),
$$

so $G(s)=s^{-u} e^{-i v \ln (s)}$. Using the Laplace transform pair,

$$
\mathcal{L}\left\{s^{-q}\right\}=\frac{t^{q-1}}{\Gamma(q)}
$$

the impulse response $\tilde{g}(t)$ of the system is found to be

$$
\begin{aligned}
\tilde{g}(t) & =\mathcal{L}^{-1}\{G(s)\}=\mathcal{L}^{-1}\left\{s^{-(u+i v)}\right\}=\frac{t^{u+i v-1}}{\Gamma(u+i v))} \\
& =\frac{t^{u-1}}{\Gamma(u+i v)} t^{i v}=\frac{t^{u-1}}{\Gamma(u+i v)} e^{i v \ln (t)} .
\end{aligned}
$$

Using Euler's identity, this can be written as

$$
\tilde{g}(t)=\mathcal{L}^{-1}\{G(s)\}=\frac{t^{u-1}}{\Gamma(u+i v)}(\cos (v \ln (t))+i \sin (v \ln (t))) .
$$

This impulse response is complex-valued for $v \neq 0$. However, it is seen in the next section that the complex-order differintegral plus its conjugate-order differintegral has a real-valued impulse response. 


\subsection{Conjugate-Order Differintegrals}

We now define the conjugated differintegral as

$$
{ }_{-\infty} d_{t}^{-u, v} f(t)={ }_{-\infty} d_{t}^{-u+i v} f(t)+{ }_{-\infty} d_{t}^{-u-i v} f(t)
$$

The Laplace transform of Equation 4.5

$$
\mathcal{L}\left\{{ }_{-\infty} d_{t}^{-u, v} f(t)\right\}=\left(s^{-u+i v}+s^{-u-i v}\right) F(s)=s^{-u}\left(s^{i v}+s^{-i v}\right) F(s),
$$

which after application of Euler's identity gives

$$
\begin{aligned}
\mathcal{L}\left\{{ }_{-\infty} d_{t}^{-u, v} f(t)\right\}= & s^{-u}\left(e^{i v \ln (s)}+e^{-i v \ln (s)}\right) F(s) \\
= & s^{-u}((\cos (v \ln (s))+i \sin (v \ln (s))) \\
& +(\cos (v \ln (s))-i \sin (v \ln (s)))) F(s) \\
= & 2 s^{-u} \cos (v \ln (s)) F(s) .
\end{aligned}
$$

Hence, the Laplace-domain representation of the conjugated differintegral is $G(s)=$ $\left(s^{-u+i v}+s^{-u-i v}\right)=2 s^{-u} \cos (v \ln (s))$. The impulse response $\tilde{g}(t)$ of this system is given by

$$
\begin{aligned}
\tilde{g}(t) & =\mathcal{L}^{-1}\{G(s)\}=\mathcal{L}^{-1}\left\{s^{-u+i v}+s^{-u-i v}\right\} \\
& =\frac{t^{u+i v-1}}{\Gamma(u+i v)}+\frac{t^{u-i v-1}}{\Gamma(u-i v)}
\end{aligned}
$$


Note that by [1],

$$
\operatorname{Re}(\Gamma(u+i v))=\operatorname{Re}(\Gamma(u-i v))
$$

and

$$
\operatorname{Im}(\Gamma(u+i v))=-\operatorname{Im}(\Gamma(u-i v)) .
$$

Hence, $\tilde{g}(t)$ can be written as

$$
\begin{aligned}
\tilde{g}(t)= & t^{u-1}\left(\operatorname{Re}\left(\frac{1}{\Gamma(u+i v)}\right) t^{i v}+i \operatorname{Im}\left(\frac{1}{\Gamma(u+i v)}\right) t^{i v}\right. \\
& \left.+\operatorname{Re}\left(\frac{1}{\Gamma(u+i v)}\right) t^{-i v}-i \operatorname{Im}\left(\frac{1}{\Gamma(u+i v)}\right) t^{-i v}\right) \\
= & t^{u-1}\left(\operatorname{Re}\left(\frac{1}{\Gamma(u+i v)}\right)\left(t^{i v}+t^{-i v}\right)+i \operatorname{Im}\left(\frac{1}{\Gamma(u+i v)}\right)\left(t^{i v}-t^{-i v}\right)\right) .
\end{aligned}
$$

We now write $t^{ \pm i v}=e^{ \pm i v \ln (t)}$ and apply Euler's identity to give

$$
\begin{aligned}
\tilde{g}(t)= & t^{u-1} \operatorname{Re}\left(\frac{1}{\Gamma(u+i v)}\right)(\cos (v \ln (t))+i \sin (v \ln (t))) \\
& +t^{u-1} \operatorname{Re}\left(\frac{1}{\Gamma(u+i v)}\right)(\cos (v \ln (t))-i \sin (v \ln (t))) \\
& +i t^{u-1} \operatorname{Im}\left(\frac{1}{\Gamma(u+i v)}\right)(\cos (v \ln (t))+i \sin (v \ln (t))) \\
& -i t^{u-1} \operatorname{Im}\left(\frac{1}{\Gamma(u+i v)}\right)(\cos (v \ln (t))-i \sin (v \ln (t))) .
\end{aligned}
$$


Hence, $\tilde{g}(t)$ is given by

$$
\begin{aligned}
\tilde{g}(t) & =\mathcal{L}^{-1}\left\{s^{-(u+i v)}+s^{-(u-i v)}\right\}=\mathcal{L}^{-1}\left\{2 s^{-u} \cos (v \ln (s))\right\} \\
& =2 t^{u-1}\left(\operatorname{Re}\left(\frac{1}{\Gamma(u+i v)}\right) \cos (v \ln (t))-\operatorname{Im}\left(\frac{1}{\Gamma(u+i v)}\right) \sin (v \ln (t))\right) .
\end{aligned}
$$

Note that this impulse response is a purely real-valued function [7].

Hence, when a complex-order operator is properly paired with its conjugateorder operator, a real-valued impulse response is created. This is analogous to a complex pole of an integer-order system. A system whose transfer function has a single complex pole has a complex-valued impulse response, but a system whose transfer function has two complex-conjugate poles has a real-valued impulse response.

\subsection{Conjugate-Order Systems}

Since conjugate-order differintegrals can produce systems with real-valued impulse responses, it is sensible to apply the same sort of process to more general operators

of complex order. Hartley and Lorenzo [33] demonstrated the Laplace transform pair

$$
\mathcal{L}\left\{F_{q}(a, t)\right\}=\frac{1}{s^{q}-a},
$$

where $F_{q}(a, t)$ is the $F$-function as described in Section 3.4.3. The derivation of the Laplace transform pair does not require that $q$ or $a$ be real-valued. Consider the 
system whose transfer function, $G(s)$, is given by

$$
G(s)=\frac{r}{s^{q}-a}+\frac{\bar{r}}{s^{\bar{q}}-\bar{a}}=\frac{r s^{\bar{q}}+\bar{r} s^{q}-(\bar{r} a+r \bar{a})}{\left(s^{q}-a\right)\left(s^{\bar{q}}-\bar{a}\right)} .
$$

Using the Laplace transform pair given in Equation 4.10, the impulse response $\tilde{g}(t)$ of the system with transfer function $G(s)$ is

$$
\tilde{g}(t)=\mathcal{L}^{-1}\{G(s)\}=r F_{q}(a, t)+\bar{r} F_{\bar{q}}(\bar{a}, t) .
$$

For $a, z \in \mathbb{C}$ and $n \in \mathbb{N}$, recall that $\overline{a^{n}}=(\bar{a})^{n}$ and $\overline{\Gamma(z)}=\Gamma(\bar{z})$. Hence, $\tilde{g}(t)$ may be written as

$$
\begin{aligned}
\tilde{g}(t) & =r \sum_{n=0}^{\infty} \frac{a^{n} t^{(n+1) q}}{\Gamma((n+1) q)}+\bar{r} \sum_{n=0}^{\infty} \frac{\bar{a}^{n} t^{(n+1) \bar{q}}}{\Gamma((n+1) \bar{q})} \\
& =\sum_{n=0}^{\infty}\left(r \frac{a^{n}}{\Gamma((n+1) q)} t^{(n+1)(u+i v)}+\bar{r} \frac{\bar{a}^{n}}{\Gamma((n+1) \bar{q})} t^{(n+1)(u-i v)}\right) \\
& =\sum_{n=0}^{\infty} t^{(n+1) u-1}\left(\Phi_{n} t^{i(n+1) v}+\overline{\Phi_{n}} t^{-i(n+1) v}\right),
\end{aligned}
$$

where

$$
\Phi_{n}=\frac{r a^{n}}{\Gamma((n+1) q)}
$$

This can be written as

$$
\tilde{g}(t)=2 \sum_{n=0}^{\infty} t^{(n+1) u-1}\left(\operatorname{Re}\left(\Phi_{n}\right) \cos ((n+1) v \ln (t))+\operatorname{Im}\left(\Phi_{n}\right) \sin ((n+1) v \ln (t))\right),
$$


which is a real-valued time function. Hence, the convolution operator associated with the transfer function in Equation 4.11 maps real-valued functions to real-valued functions. We call the system associated with Equation 4.11 the fundamental linear conjugate-order system. We now state this result formally.

Theorem 4.3.1 The system with transfer function

$$
G(s)=\frac{r}{s^{q}-a}+\frac{\bar{r}}{s^{\bar{q}}-\bar{a}}
$$

has a real-valued impulse response.

Proof See above.

\subsubsection{Poles of the Fundamental Linear Conjugate-Order System}

In order to gain an understanding of the system, it is desirable to find the poles of the system. Let $x$ and $y$ be given by

$$
x=\frac{u}{u^{2}+v^{2}}
$$

and

$$
y=\frac{v}{u^{2}+v^{2}} .
$$


Then

$$
\frac{1}{u \pm i v}=x \mp i y
$$

The poles of $G(s)$ are given by

$$
s=a^{\frac{1}{u+i v}}=a^{x-i y}
$$

and

$$
s=(\bar{a})^{\frac{1}{u-v}}=(\bar{a})^{x+i y} .
$$

First, consider the roots given by Equation 4.15. Note that a can be written as

$$
a=|a| e^{i(\operatorname{Arg}(a)+2 \pi k)}=e^{\ln |a|+i(\operatorname{Arg}(a)+2 \pi k)}
$$

and $\bar{a}$ as

$$
\bar{a}=|a| e^{-i(\operatorname{Arg}(a)+2 \pi k)}=e^{\ln |a|-i(\operatorname{Arg}(a)+2 \pi k)} .
$$

Substituting $a$ into Equation 4.15 results in

$$
s=\left(e^{\ln |a|+i(\operatorname{Arg}(a)+2 \pi k)}\right)^{x-i y}=e^{x \ln |a|+y \operatorname{Arg}(a)+y 2 \pi k} e^{i(x \operatorname{Arg}(a)+x 2 \pi k-y \ln |a|)} .
$$

Similarly, substituting $\bar{a}$ into Equation 4.16 results in

$$
s=\left(e^{\ln |a|-i(\operatorname{Arg}(a)+2 \pi k)}\right)^{x+i y}=e^{x \ln |a|+y \operatorname{Arg}(a)+y 2 \pi k} e^{-i(x \operatorname{Arg}(a)+x 2 \pi k-y \ln |a|)},
$$


which gives the complex conjugates of the poles given by Equation 4.17.

We consider now the poles that lie on the primary Riemann sheet, which are those whose angles lie between $-\pi$ and $\pi$. This means that

$$
-\pi<x \operatorname{Arg}(a)+x 2 \pi k-y \ln |a|<\pi
$$

Theorem 4.3.2 If $\operatorname{Re}(q) \neq 0$, then $G(s)=\frac{r}{s^{q}-a}+\frac{\bar{r}}{s^{\bar{q}}-\bar{a}}$ has a finite number of poles on the primary Riemann sheet.

Proof Since the poles of $\frac{\bar{r}}{s^{\bar{q}}-\bar{a}}$ are the complex conjugates of the poles of $\frac{r}{s^{q}-a}$, it suffices to show that $\frac{r}{s^{q}-a}$ has a finite number of poles on the primary Riemann sheet. Consider $s_{k}$ a pole of $\frac{r}{s^{q}-a}$ which is given by Equation 4.18 as

$$
s_{k}=\left|s_{k}\right| e^{i \arg \left(s_{k}\right)}=e^{x \ln |a|+y \operatorname{Arg}(a)+y 2 \pi k} e^{i(x \operatorname{Arg}(a)+x 2 \pi k-y \ln |a|)} .
$$

Thus $s_{k}$ is on the primary Riemann sheet if and only if

$$
\frac{y \ln |a|-x \operatorname{Arg}(a)-\pi}{2 \pi}<x k<\frac{y \ln |a|-x \operatorname{Arg}(a)+\pi}{2 \pi} .
$$

For $u=\operatorname{Re}(q) \neq 0, x \neq 0$, so the requirement given in Equation 4.20 can be written as

$$
\frac{y \ln |a|-x \operatorname{Arg}(a)-\pi}{x 2 \pi}<k<\frac{y \ln |a|-x \operatorname{Arg}(a)+\pi}{x 2 \pi} .
$$


If there is no $k$ that satisfies Equation 4.21, then $G(s)$ has no poles on the primary Riemann sheet. If there is a $k$ that satisfies Equation 4.21 , then $k$ is an element of an open bounded interval. Therefore there are a finite number of integers $k$ such that $s_{k}$ is on the primary Riemann sheet.

We will now consider the conditions under which a conjugate-order system is stable and causal.

\subsection{Stable, Causal Conjugate-Order Systems}

We will show in Section 5.5.2 that a transfer function corresponds to a stable, causal system when it is analytic in the right-half plane of the primary Riemann sheet. The only singularities that the fundamental linear conjugate-order system can have in the right-half plane of the primary Riemann sheet are poles. Hence, we will say that the transfer function of the fundamental linear conjugate-order system is analytic in the right half plane of he primary Riemann sheet if it has no poles there. We now consider how many poles such a transfer function may have on the primary Riemann sheet.

Theorem 4.4.1 If $\operatorname{Re}(q) \neq 0$ and $G(s)=\frac{r}{s^{q}-a}+\frac{\bar{r}}{s^{\bar{q}}-\bar{a}}$ is analytic in the right half plane of the primary Riemann sheet, then $G(s)$ has at most four poles on the primary Riemann sheet. 
Proof Since the poles of $\frac{\bar{r}}{s^{\bar{q}}-\bar{a}}$ are the complex conjugates of the poles of $\frac{r}{s^{q}-a}$, it suffices to show that $\frac{r}{s^{q}-a}$ has at most two poles on the primary Riemann sheet. Consider $s_{k}$ as given in Equation 4.19. The argument of $s_{k}$, given by

$$
\arg \left(s_{k}\right)=x \operatorname{Arg}(a)+x 2 \pi k-y \ln |a|,
$$

is linear in $k$. Thus, by Equation 4.22, $\left|\arg \left(s_{k+1}\right)-\arg \left(s_{k}\right)\right|$ is constant, i.e.

$$
\left|\arg \left(s_{k+1}\right)-\arg \left(s_{k}\right)\right|=|x| \cdot 2 \pi \text {. }
$$

Because $G(s)$ is assumed to be analytic in the right half plane of the primary Riemann sheet, there is no value of $k$ for which $-\frac{\pi}{2}<\arg \left(s_{k}\right)<\frac{\pi}{2}$. Therefore,

$$
\left|\arg \left(s_{k+1}\right)-\arg \left(s_{k}\right)\right|>\pi
$$

for any $k \in \mathbb{Z}$. Suppose that there are $M$ poles of $\frac{r}{s^{q}-a}$, on the primary Riemann sheet. This means that

$$
\left|\arg \left(s_{k+M}\right)-\arg \left(s_{k}\right)\right|<2 \pi
$$

However, for a stable system, Equation 4.24 gives

$$
\left|\arg \left(s_{k+M}\right)-\arg \left(s_{k}\right)\right|=\sum_{m=1}^{M-1}\left|\arg \left(s_{m+1}\right)-\arg \left(s_{m}\right)\right|>\sum_{m=1}^{M-1} \pi=(M-1) \pi .
$$


Thus,

$$
(M-1) \pi<\left|\arg \left(s_{k+M}\right)-\arg \left(s_{k}\right)\right|<2 \pi,
$$

which implies

$$
(M-1)<2
$$

or

$$
M<3 .
$$

Thus if $\frac{r}{s^{q}-a}$ is stable, it has at most two poles on the primary Riemann sheet. Since the poles of $\frac{\bar{r}}{s^{\bar{q}}-\bar{a}}$ are the complex conjugates of the poles of $\frac{r}{s^{q}-a}, \frac{r}{s^{q}-a}+\frac{\bar{r}}{s^{\bar{q}}-\bar{a}}$ has at most four poles on the primary Riemann sheet.

Corollary 4.4.2 Let $q \in \mathbb{C} \backslash\{0\}, G(s)=\frac{r}{s^{q}-a}+\frac{\bar{r}}{s^{\bar{q}}-\bar{a}}, u=\operatorname{Re}(q)$, and $v=\operatorname{Im}(q)$. A necessary condition for $G(s)$ to be stable and causal is that $q=u+i v$ must satisfy either

$$
\begin{gathered}
u=0, \\
(u-1)^{2}+v^{2}<1,
\end{gathered}
$$

or

$$
(u+1)^{2}+v^{2}<1
$$


Proof Suppose that $u \neq 0$. By Equation 4.23 and Equation 4.24 if $u \neq 0$ and $G(s)$ is stable, $2|x|>1$. Thus, either $2 x>1$ or $-2 x>1$. Since $x=\frac{u}{u^{2}+v^{2}}, q=u+i v$ satisfies either

$$
u^{2}+v^{2}<2 u
$$

or

$$
u^{2}+v^{2}<-2 u
$$

Completing the square on both Equations 4.28 and 4.29 gives the desired result.

\subsubsection{Stable, Causal $G(s)$ for a Fixed $a$}

Next we derive necessary and sufficient conditions for $G(s)=\frac{r}{s^{q}-a}+\frac{\bar{r}}{s^{\bar{q}}-\bar{a}}$ to correspond to a stable, causal system.

Theorem 4.4.3 For $q \in \mathbb{C} \backslash\{0\}, u=\operatorname{Re}(q), v=\operatorname{Im}(q)$, and $a \in \mathbb{C}$, define

$$
\begin{gathered}
u_{k}(a)=\frac{\operatorname{Arg}(a)+2 \pi k}{\pi}, \\
v_{k}(a)=\frac{\ln |a|}{\pi}, \\
D_{k}^{+}(a)=\left\{u+i v \in \mathbb{C}:\left(u-u_{k}\right)^{2}+\left(v+v_{k}\right)^{2}<u_{k}^{2}+v_{k}^{2}\right\},
\end{gathered}
$$


and

$$
D_{k}^{-}(a)=\left\{u+i v \in \mathbb{C}:\left(u+u_{k}\right)^{2}+\left(v-v_{k}\right)^{2}<u_{k}^{2}+v_{k}^{2}\right\}
$$

Then $G(s)=\frac{r}{s^{q}-a}+\frac{\bar{r}}{s^{\bar{q}}-\bar{a}}$ corresponds to a stable, causal system if and only if

$$
q \in \bigcap_{k \in \mathbb{Z}}\left(D_{k}^{+}(a) \cup D_{k}^{-}(a)\right)
$$

Proof Recall that the poles of $\frac{\bar{r}}{s^{q}-\bar{a}}$ are the complex-conjugates of the poles of $\frac{r}{s^{q}-a}$, so we only need to consider the poles $\left\{s_{k}\right\}$ of $\frac{r}{s^{q}-a}$. The argument of $s_{k}$ is given by Equation 4.22 as

$$
\begin{aligned}
\arg \left(s_{k}\right) & =x \operatorname{Arg}(a)+x 2 \pi k-y \ln |a| \\
& =\frac{u \operatorname{Arg}(a)+u 2 \pi k-y \ln |a|}{u^{2}+v^{2}} .
\end{aligned}
$$

$G(s)$ corresponds to a stable, causal system if and only if the right-half plane on the primary Riemann sheet is analytic. That is, $G(s)$ corresponds to a stable, causal system if and only if for every $k \in \mathbb{Z}$, either

$$
\frac{u \operatorname{Arg}(a)+u 2 \pi k-v \ln |a|}{u^{2}+v^{2}}>\frac{\pi}{2}
$$

or

$$
\frac{u \operatorname{Arg}(a)+u 2 \pi k-v \ln |a|}{u^{2}+v^{2}}<-\frac{\pi}{2}
$$


holds. Multiplying both sides of the inequality in Equation 4.30 by $\frac{2}{\pi}\left(u^{2}+v^{2}\right)>0$ gives

$$
2 u\left(\frac{\operatorname{Arg}(a)+2 \pi k}{\pi}\right)-2 v \frac{\ln |a|}{\pi}>u^{2}+v^{2}
$$

Bringing each term to the right of the inequality gives

$$
0>u^{2}-2 u \frac{\operatorname{Arg}(a)+2 \pi k}{\pi}+v^{2}+2 v \frac{\ln |a|}{\pi} .
$$

Completing the square for both $u$ and $v$ gives

$$
\begin{aligned}
u^{2} & -2 u \frac{\operatorname{Arg}(a)+2 \pi k}{\pi}+\left(\frac{\operatorname{Arg}(a)+2 \pi k}{\pi}\right)^{2}+v^{2}+2 v \frac{\ln |a|}{\pi}+\left(\frac{\ln |a|}{\pi}\right)^{2} \\
& <\left(\frac{\ln |a|}{\pi}\right)^{2}+\left(\frac{\operatorname{Arg}(a)+2 \pi k}{\pi}\right)^{2}
\end{aligned}
$$

which simplifies to

$$
\left(u-\frac{\operatorname{Arg}(a)+2 \pi k}{\pi}\right)^{2}+\left(v+\frac{\ln |a|}{\pi}\right)^{2}<\left(\frac{\operatorname{Arg}(a)+2 \pi k}{\pi}\right)^{2}+\left(\frac{\ln |a|}{\pi}\right)^{2}
$$

Considering this relation in the $(u, v)$ plane, this gives an open disk centered at

$$
\frac{\operatorname{Arg}(a)+2 \pi k}{\pi}-i \frac{\ln |a|}{\pi}=u_{k}-i v_{k}
$$

with radius, $\rho_{k}$, given by

$$
\rho_{k}=\frac{\sqrt{(\operatorname{Arg}(a)+2 \pi k)^{2}+\ln ^{2}|a|}}{\pi}=\left|u_{k}+i v_{k}\right|
$$


The set of $q \in \mathbb{C}$ that satisfy Equation 4.32 for a specific $k \in \mathbb{Z}$ is the set of $q$ such that $q \in D_{k}^{+}(a)$.

Similarly, Equation 4.31 can be written as

$$
\left(u+\frac{\operatorname{Arg}(a)+2 \pi k}{\pi}\right)^{2}+\left(v-\frac{\ln |a|}{\pi}\right)^{2}<\left(\frac{\operatorname{Arg}(a)+2 \pi k}{\pi}\right)^{2}+\left(\frac{\ln |a|}{\pi}\right)^{2},
$$

which is an open disk centered at

$$
-\frac{\operatorname{Arg}(a)+2 \pi k}{\pi}+i \frac{\ln |a|}{\pi}=-u_{k}+i v_{k}
$$

with radius, $\rho_{k}$, given by

$$
\rho_{k}=\frac{\sqrt{(\operatorname{Arg}(a)+2 \pi k)^{2}+\ln ^{2}|a|}}{\pi}=\left|u_{k}+i v_{k}\right|
$$

The set of $q \in \mathbb{C}$ that satisfy Equation 4.33 for a specific $k \in \mathbb{Z}$ is the set of $q$ such that $q \in D_{k}^{-}(a)$.

Given $a \in \mathbb{C}$, the set of $q$ such that $G(s)$ corresponds to a stable, causal system is $q \in \mathcal{Q}$, where

$$
\mathcal{Q}=\bigcap_{k \in \mathbb{Z}}\left(D_{k}^{+}(a) \cup D_{k}^{-}(a)\right)
$$


Note that if $|a| \neq 1$, then part of the imaginary axis is in the set $\mathcal{Q}=\bigcap_{k \in \mathbb{Z}}\left(D_{k}^{+}(a) \cup D_{k}^{-}(a)\right)$. For such $a$, if the center of $D_{k}^{ \pm}(a)$ is in the upper-right quarter plane, then a portion of $D_{k}^{ \pm}(a)$ extends into the upper-left quarter plane and contains an interval on the imaginary axis. The smallest of these disks determines the length of that interval. As $k$ is varied to increase the size of $D_{k}^{ \pm}(a)$, depth into which the disk enters the upper-left quarter plane decreases. For any point $s_{0}$ in the upper-left quarter plane, we may find a $k$ such that $D_{k}^{ \pm}(a)$ whose center is in the upper-right quarter plane does not contain $s_{0}$. We can make a similar argument for disks centered in the upper-left, lower-left, and lower-right quarter planes. For $v \neq 0$, there is a small $u$ such that $i v \in \mathcal{Q}$ but $u+i v \notin \mathcal{Q}$.

Note that in the notation of Theorem 4.4.3, Corollary 4.4.2 states that a necessary condition for $G(s)$ to correspond to a stable, causal system is that $q \in$ $\left(D_{0}^{+}(-1) \cup D_{0}^{-}(-1) \cup i \mathbb{R}\right)$. We now show that Theorem 4.4.3 does not contradict Corollary 4.4.2.

Theorem 4.4.4 For $q \in \mathbb{C} \backslash\{0\}, u=\operatorname{Re}(q), v=\operatorname{Im}(q)$, and $a \in \mathbb{C}$, define

$$
\begin{gathered}
u_{k}(a)=\frac{\operatorname{Arg}(a)+2 \pi k}{\pi}, \\
v_{k}(a)=\frac{\ln |a|}{\pi}, \\
D_{k}^{+}(a)=\left\{u+i v \in \mathbb{C}:\left(u-u_{k}\right)^{2}+\left(v+v_{k}\right)^{2}<u_{k}^{2}+v_{k}^{2}\right\},
\end{gathered}
$$


and

$$
D_{k}^{-}(a)=\left\{u+i v \in \mathbb{C}:\left(u+u_{k}\right)^{2}+\left(v-v_{k}\right)^{2}<u_{k}^{2}+v_{k}^{2}\right\}
$$

Then

$$
\bigcap_{k \in \mathbb{Z}}\left(D_{k}^{+}(a) \cup D_{k}^{-}(a)\right) \subset\left(D_{0}^{+}(-1) \cup D_{0}^{-}(-1) \cup i \mathbb{R}\right)
$$

Proof Suppose $q \notin\left(D_{0}^{+}(-1) \cup D_{0}^{-}(-1) \cup i \mathbb{R}\right)$. Then $-\frac{1}{2} \leq \frac{u}{u^{2}+v^{2}} \leq \frac{1}{2}$ and $u \neq 0$. If $u>0$, then $\frac{u^{2}+v^{2}}{2 u} \geq 1 \geq-\frac{u^{2}+v^{2}}{2 u}$. If $u<0$, then $\frac{u^{2}+v^{2}}{2 u} \leq-1 \leq-\frac{u^{2}+v^{2}}{2 u}$. Define $m$ by

$$
m=\frac{v \ln |a|-u \operatorname{Arg}(a)}{\pi\left(u^{2}+v^{2}\right)}
$$

If $u>0$, choose $k_{0} \in \mathbb{Z}$ such that $\frac{m\left(u^{2}+v^{2}\right)}{2 u} \leq k_{0} \leq \frac{(m+1)\left(u^{2}+v^{2}\right)}{2 u}$. Such a $k_{0}$ exists because $\frac{(m+1)\left(u^{2}+v^{2}\right)}{2 u}-\frac{m\left(u^{2}+v^{2}\right)}{2 u}=\frac{u^{2}+v^{2}}{2 u}>1$. If $u<0$ choose $k_{0} \in \mathbb{Z}$ such that $\frac{(m+1)\left(u^{2}+v^{2}\right)}{2 u} \leq k_{0} \leq \frac{m\left(u^{2}+v^{2}\right)}{2 u}$. Such a $k_{0}$ exists because $\frac{m\left(u^{2}+v^{2}\right)}{2 u}-\frac{(m+1)\left(u^{2}+v^{2}\right)}{2 u}=$ $\frac{u^{2}+v^{2}}{2 u}<-1$. Hence there exists a $k_{0} \in \mathbb{Z}$ such that

$$
m \leq \frac{2 u}{u^{2}+v^{2}} k_{0} \leq m+1
$$

or

$$
\pi\left(m-\frac{1}{2}\right) \leq \frac{2 \pi u}{u^{2}+v^{2}} k+0 \leq \pi\left(m+\frac{1}{2}\right)
$$

Rearranging Equation 4.34 gives

$$
-\frac{\pi}{2} \leq \frac{2 \pi u k_{0}}{u^{2}+v^{2}}-m \leq \frac{\pi}{2}
$$


which can be rewritten as

$$
-\frac{\pi}{2} \leq \frac{u \operatorname{Arg}(a)+u 2 \pi k_{0}-v \ln |a|}{u^{2}+v^{2}} \leq \frac{\pi}{2}
$$

Hence, there exists a $k_{0} \in \mathbb{Z}$ such that $q=u+i v$ satisfies neither Equation 4.30 nor Equation 4.31. Thus, $q \notin\left(D_{k_{0}}^{+}(a) \cup D_{k_{0}}^{-}(a)\right)$. Subsequently,

$$
\bigcap_{k \in \mathbb{Z}}\left(D_{k}^{+}(a) \cup D_{k}^{-}(a)\right) \subset\left(D_{0}^{+}(-1) \cup D_{0}^{-}(-1)\right) .
$$

None of the discussion in this section has considered the case where $q=0$.

In this case $G(s)=\frac{r}{1-a}+\frac{\bar{r}}{1-\bar{a}}$. For $a \neq 1, G(s)$ is a constant, and $\mathcal{G}$ multiplies inputs by that constant. This is a stable system.

\subsubsection{Examples}

We now apply Theorem 4.4.3 to several examples. We show the set of $q \in \mathbb{C}$ for which $G(s)=\frac{r}{s^{q}-a}+\frac{\bar{r}}{s^{\bar{q}}-\bar{a}}$ corresponds to a stable, causal system for a given $a \in \mathbb{C}$. In the figures to follow, the boundaries of $D_{0}^{+}(-1)$ and $D_{0}^{-}(-1)$ are depicted with dashed lines. This is to show that universal boundary for $G(s)$ to correspond to a stable, causal system for $q \notin i \mathbb{R}$. 


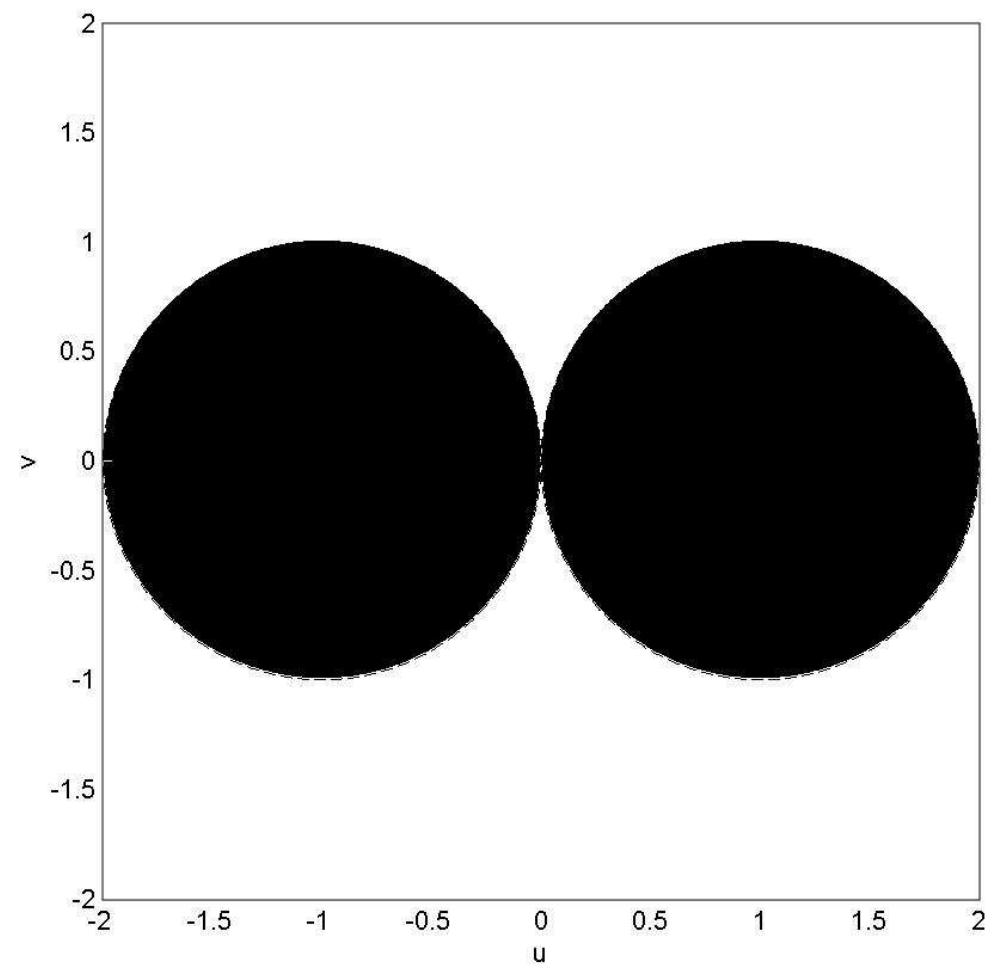

Figure 4.1: Set of $q=u+i v$ for which the system $G(s)=\frac{r}{s^{q}-\left(e^{i \pi}\right)}+\frac{\bar{r}}{s^{\bar{q}}-\left(e^{-i \pi}\right)}$ is stable and causal.

Figure 4.1 shows the region of $q \in \mathbb{C}$ such that $G(s)=\frac{r}{s^{q}-\left(e^{i \pi}\right)}+\frac{\bar{r}}{s^{\bar{q}}-\left(e^{-i \pi}\right)}$ corresponds to a stable causal system. For this system all $q$ such that $G(s)$ corresponds to a stable, causal system are in either $D_{0}^{+}(-1)$ or $D_{0}^{-}(-1)$.

Figures $4.2,4.3,4.4,4.5,4.6,4.7,4.8,4.9,4.10,4.11,4.12$, and 4.13 show the set of $q$ for which $G(s)=\frac{r}{s^{q}-\left(|a| e^{i \pi}\right)}+\frac{\bar{r}}{s^{\bar{q}}-\left(|a| e^{-i \pi}\right)}$ corresponds to a stable, causal system for $|a|=2^{ \pm k}$ for $1 \leq k \leq 12$. We will call the open set including part of the $u$-axis the primary lobe. We note several trends as $|a|$ differs more from unity. As $|a|$ gets farther from unity, the size of the primary lobe decreases. Also non-primary lobes 
appear and grow larger as $|a|$ varies from unity. We also note in these figures that for $|a|>16$, we have not included a portion of the $v$-axis where $q$ admits a stable, causal $G(s)$ because we prefer to keep the scaling the same on each of the figures.

Figures $4.13,4.13,4.13$, and 4.13 show the set of $q$ for which the transfer function $G(s)=\frac{r}{s^{q}-\left(4096 e^{i \operatorname{Arg}(a)}\right)}+\frac{\bar{r}}{s^{\bar{q}}-\left(4096 e^{-i \operatorname{Arg}(a)}\right)}$ corresponds to a stable, causal system for $\operatorname{Arg}(a)=\pi, \operatorname{Arg}(a)=\frac{\pi}{2}, \operatorname{Arg}(a)=0$, and $\operatorname{Arg}(a)=-\frac{\pi}{2}$, respectively. As the argument of $a$ decreases, the primary and other lobes seem to rotate in the $(u, v)$ plane.

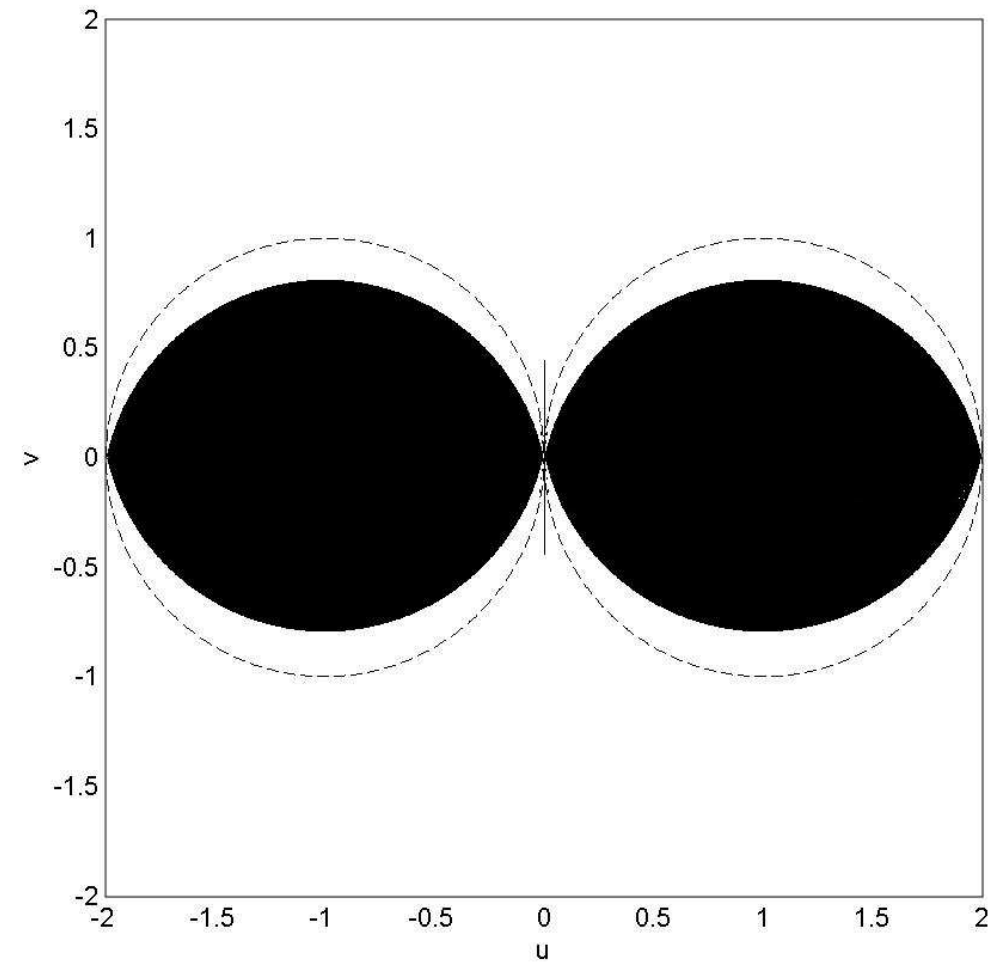

Figure 4.2: Set of $q=u+i v$ for which the system $G(s)=\frac{r}{s^{q}-\left(2 e^{i \pi}\right)}+\frac{\bar{r}}{s^{\bar{q}}-\left(2 e^{-i \pi}\right)}$ is stable and causal. 


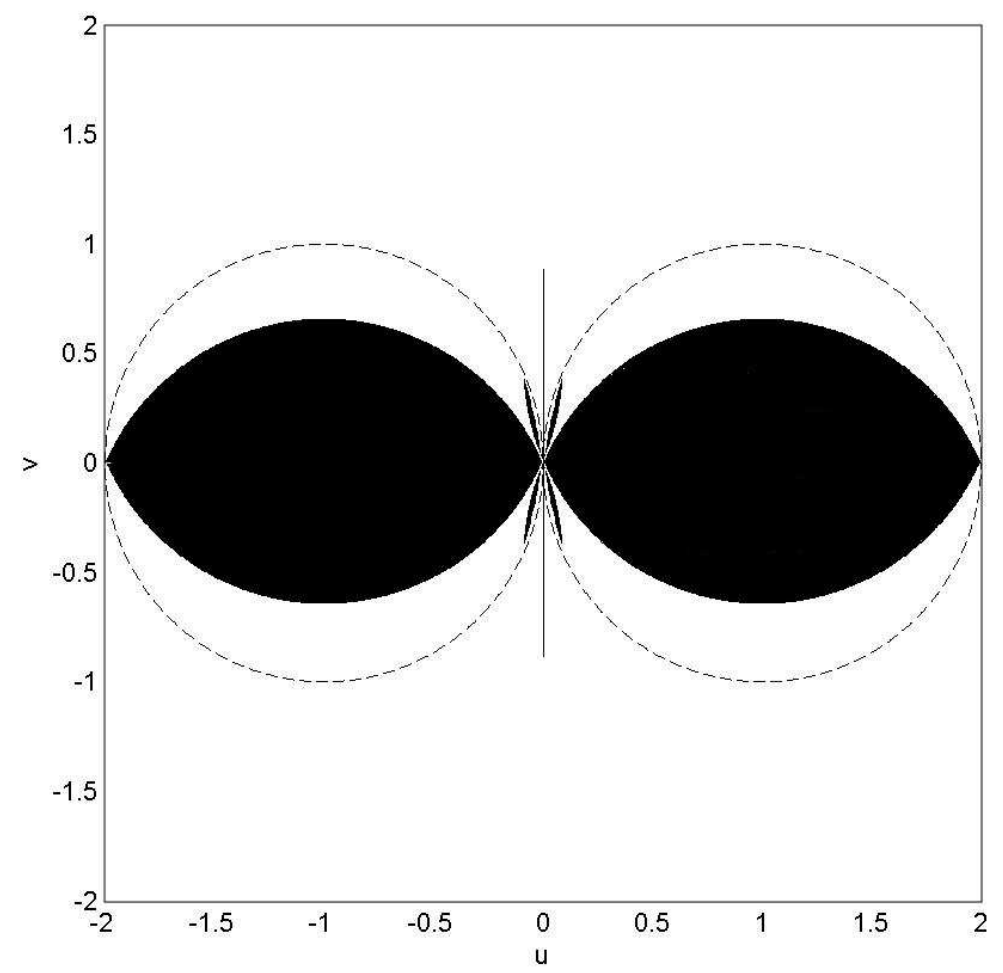

Figure 4.3: Set of $q=u+i v$ for which the system $G(s)=\frac{r}{s^{q}-\left(4 e^{i \pi}\right)}+\frac{\bar{r}}{s^{\bar{q}}-\left(4 e^{-i \pi}\right)}$ is stable and causal. 


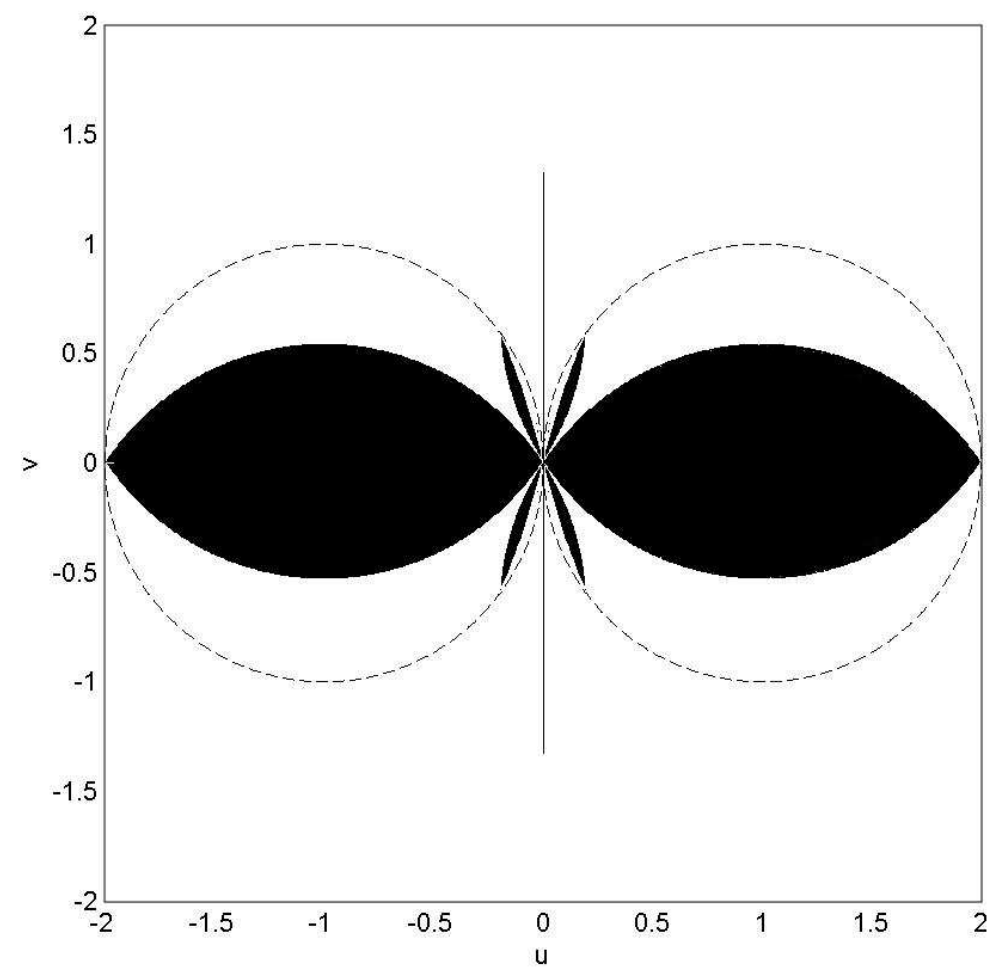

Figure 4.4: Set of $q=u+i v$ for which the system $G(s)=\frac{r}{s^{q}-\left(8 e^{i \pi}\right)}+\frac{\bar{r}}{s^{\bar{q}}-\left(8 e^{-i \pi}\right)}$ is stable and causal. 


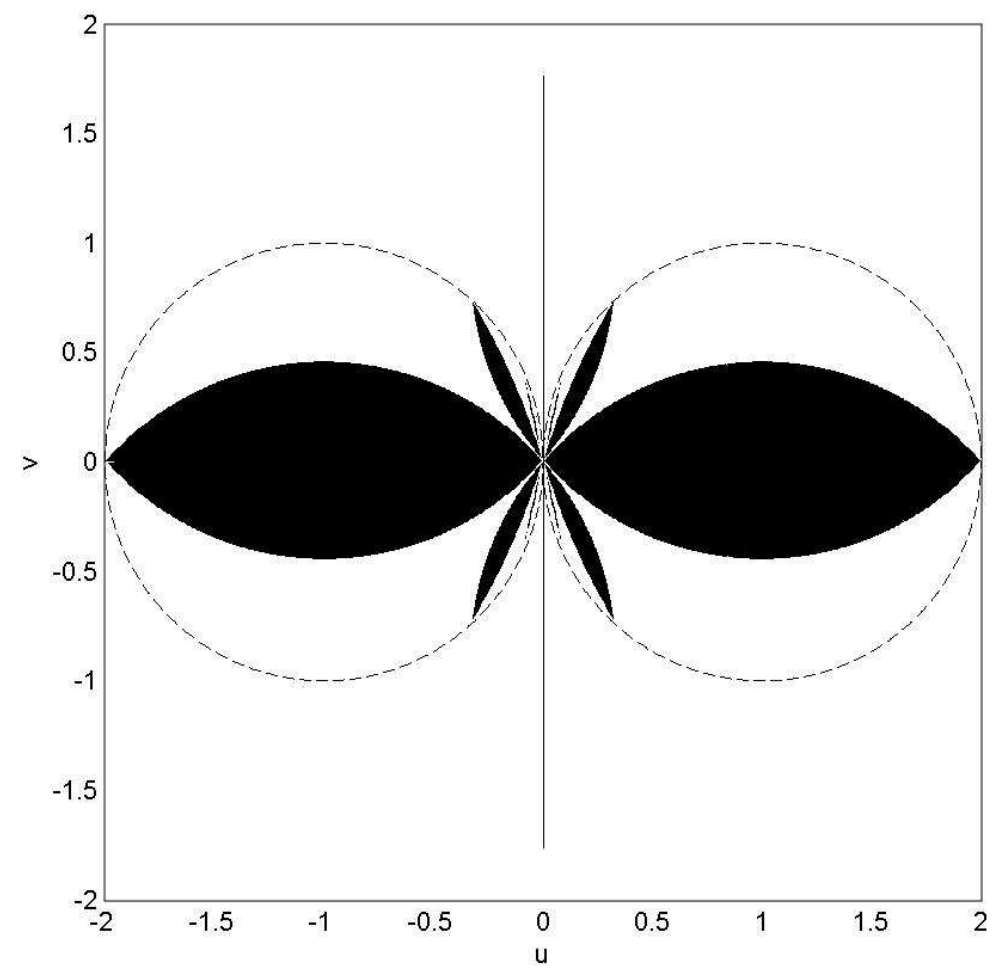

Figure 4.5: Set of $q=u+i v$ for which the system $G(s)=\frac{r}{s^{q}-\left(16 e^{i \pi}\right)}+\frac{\bar{r}}{s^{\bar{q}}-\left(16 e^{-i \pi}\right)}$ is stable and causal. 


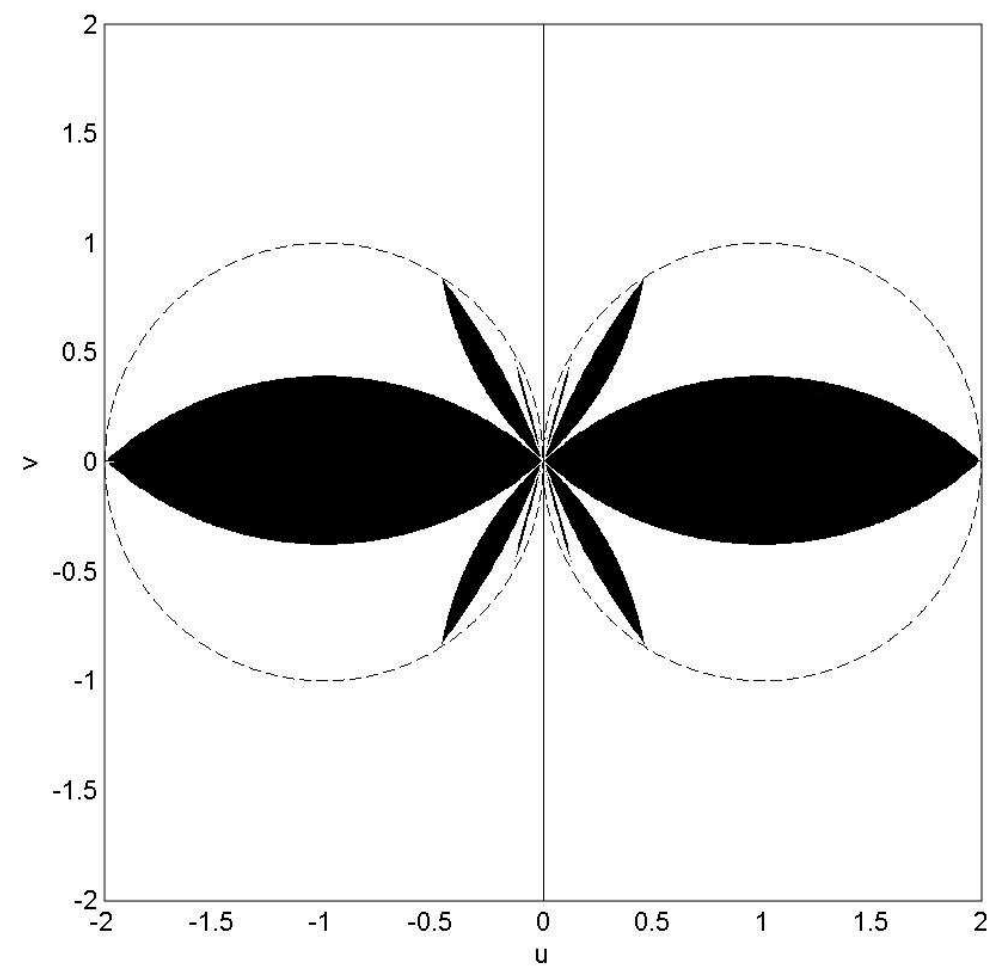

Figure 4.6: Set of $q=u+i v$ for which the system $G(s)=\frac{r}{s^{q}-\left(32 e^{i \pi}\right)}+\frac{\bar{r}}{s^{\bar{q}}-\left(32 e^{-i \pi}\right)}$ is stable and causal. 


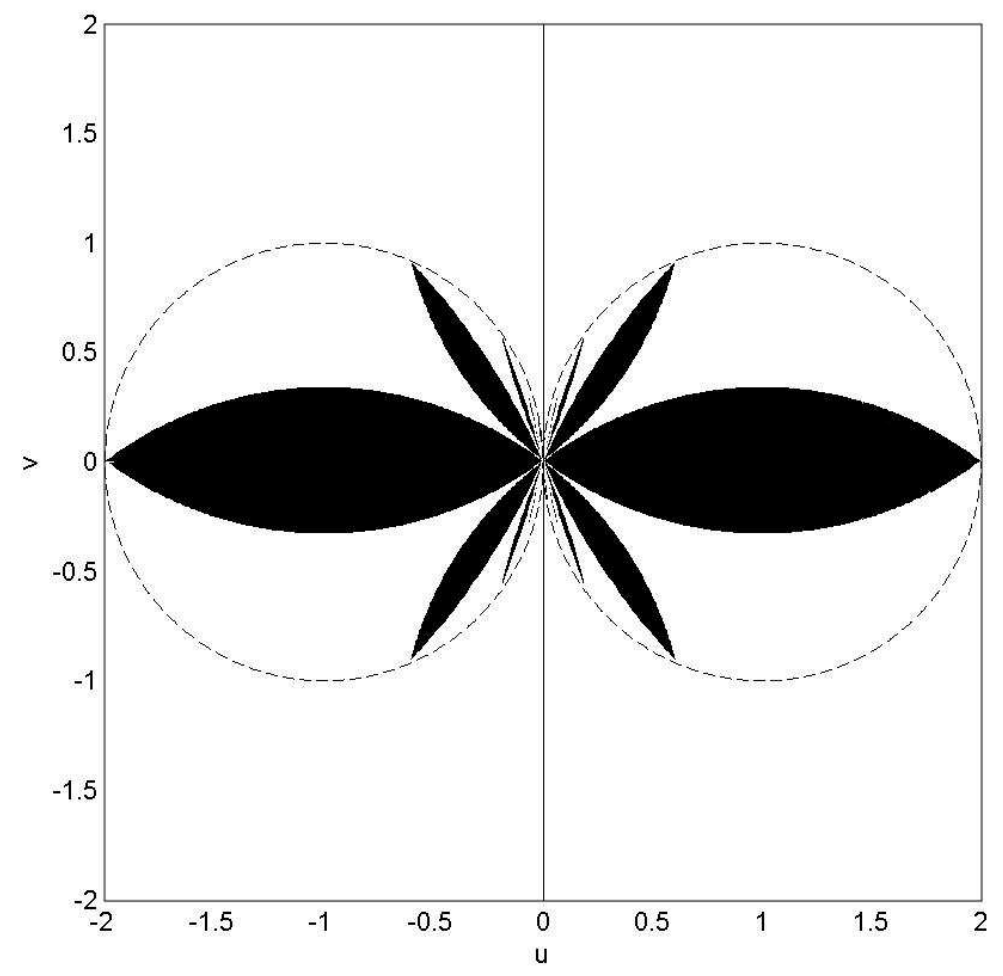

Figure 4.7: Set of $q=u+i v$ for which the system $G(s)=\frac{r}{s^{q}-\left(64 e^{i \pi}\right)}+\frac{\bar{r}}{s^{\bar{q}}-\left(64 e^{-i \pi}\right)}$ is stable and causal. 


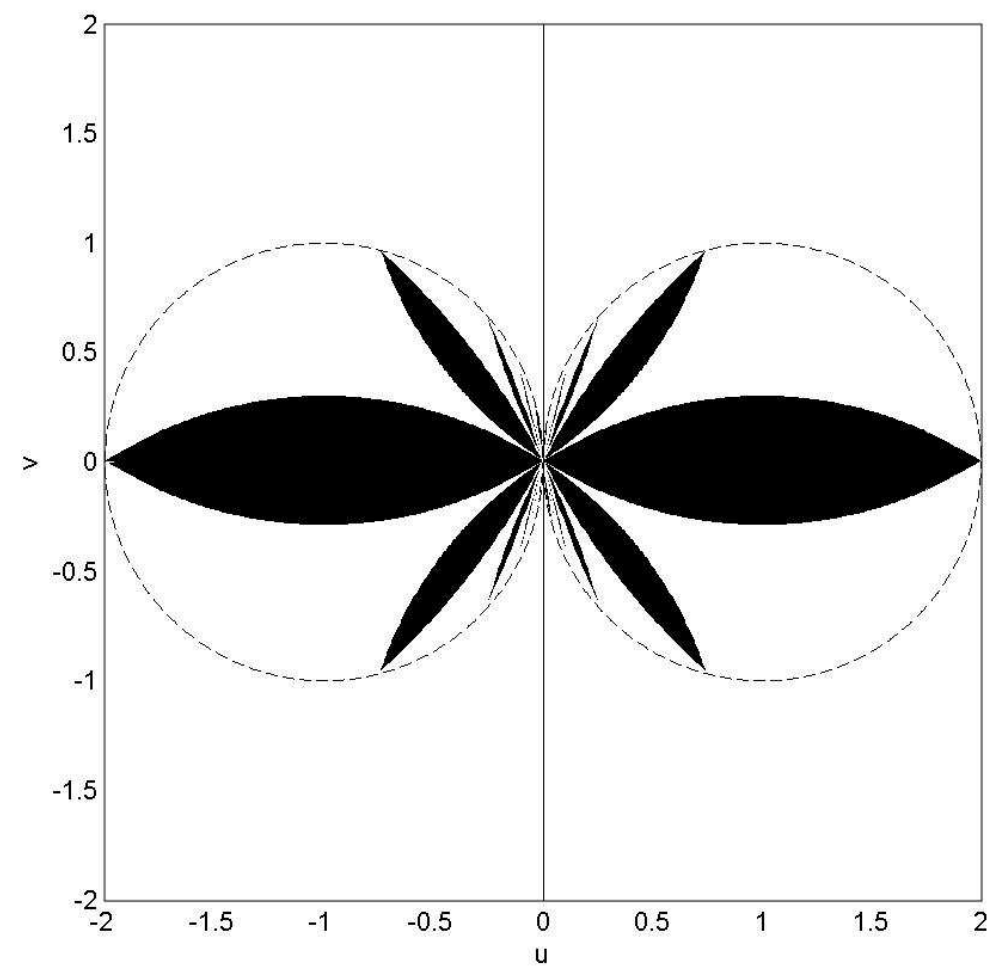

Figure 4.8: Set of $q=u+i v$ for which the system $G(s)=\frac{r}{s^{q}-\left(128 e^{i \pi}\right)}+\frac{\bar{r}}{s^{\bar{q}}-\left(128 e^{-i \pi}\right)}$ is stable and causal. 


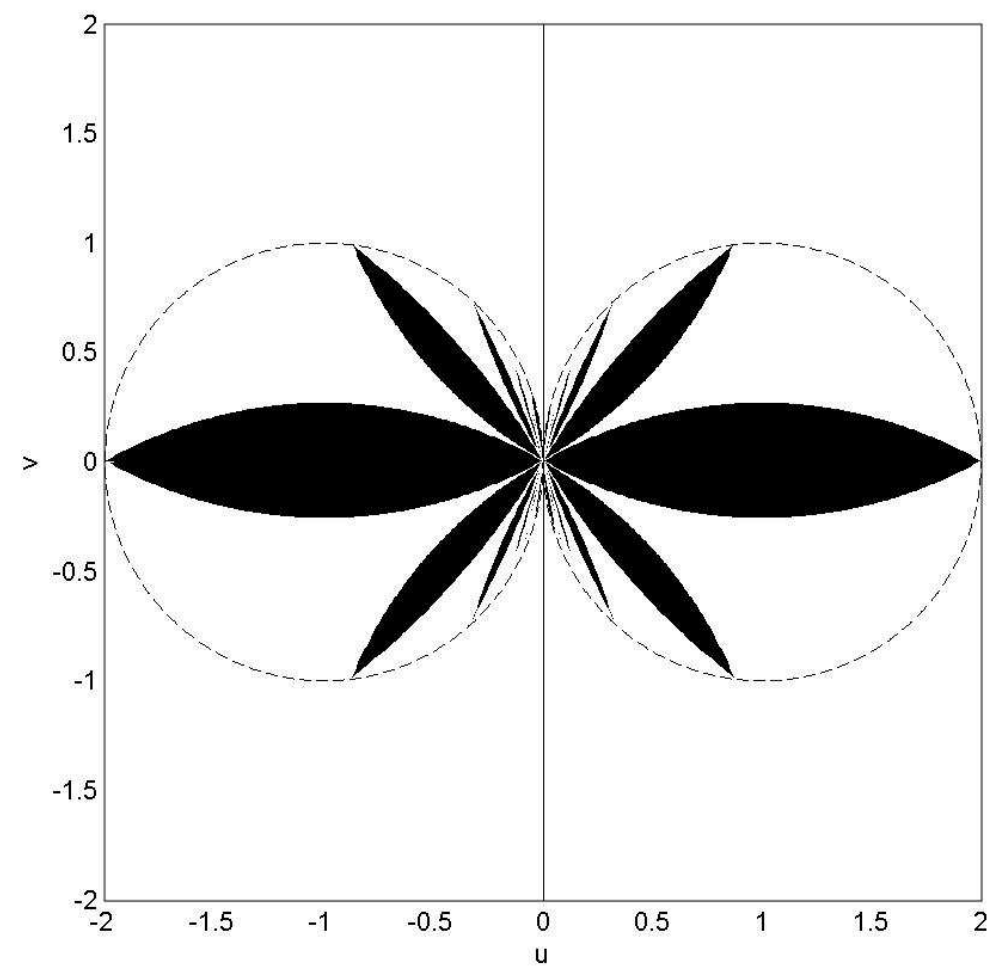

Figure 4.9: Set of $q=u+i v$ for which the system $G(s)=\frac{r}{s^{q}-\left(256 e^{i \pi}\right)}+\frac{\bar{r}}{s^{\bar{q}}-\left(256 e^{-i \pi}\right)}$ is stable and causal. 


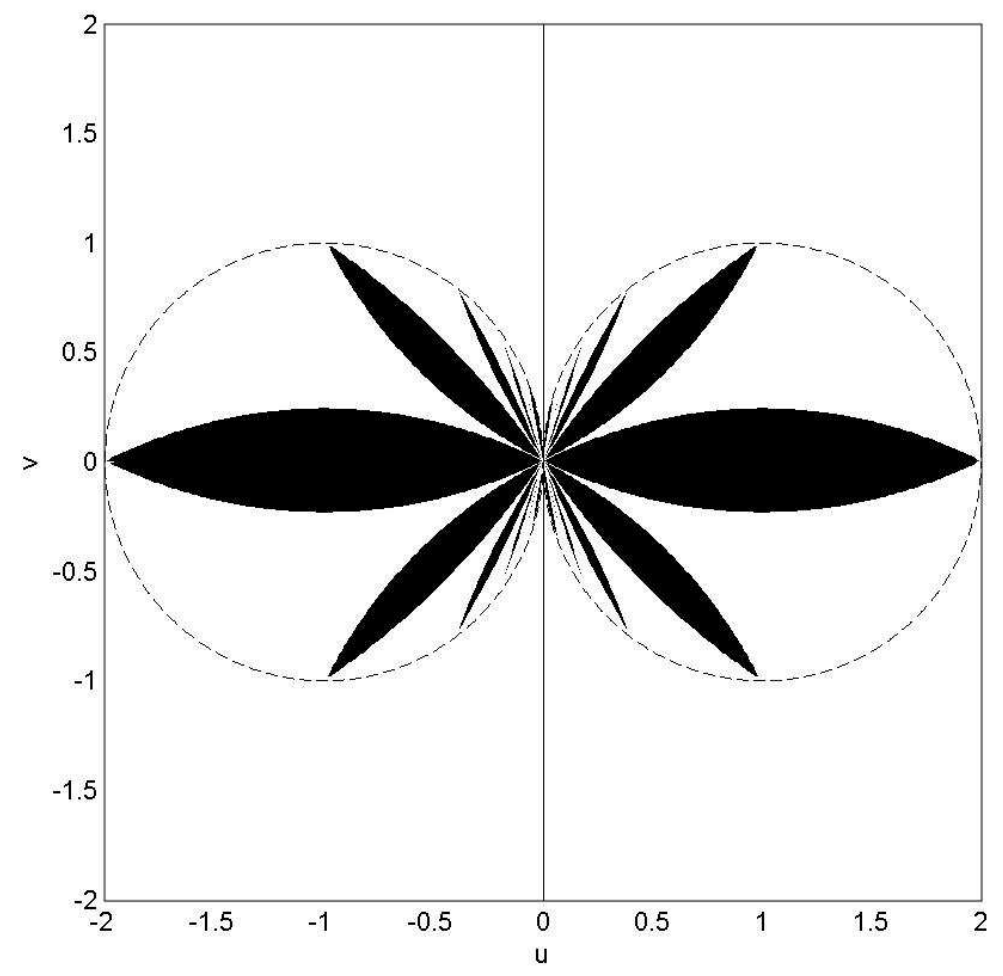

Figure 4.10: Set of $q=u+i v$ for which the system $G(s)=\frac{r}{s^{q}-\left(512 e^{i \pi}\right)}+\frac{\bar{r}}{s^{\bar{q}}-\left(512 e^{-i \pi}\right)}$ is stable and causal. 


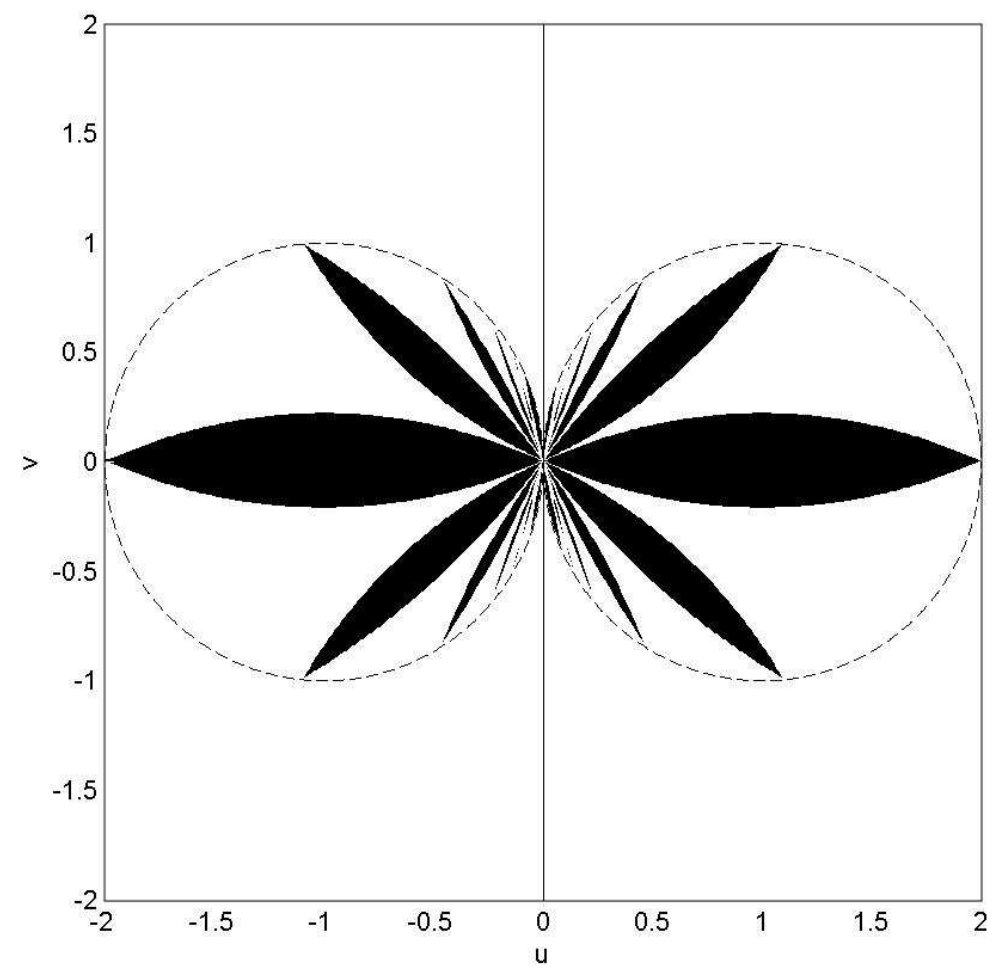

Figure 4.11: Set of $q=u+i v$ for which the system $G(s)=\frac{r}{s^{q}-\left(1024 e^{i \pi}\right)}+\frac{\bar{r}}{s^{\bar{q}}-\left(1024 e^{-i \pi}\right)}$ is stable and causal. 


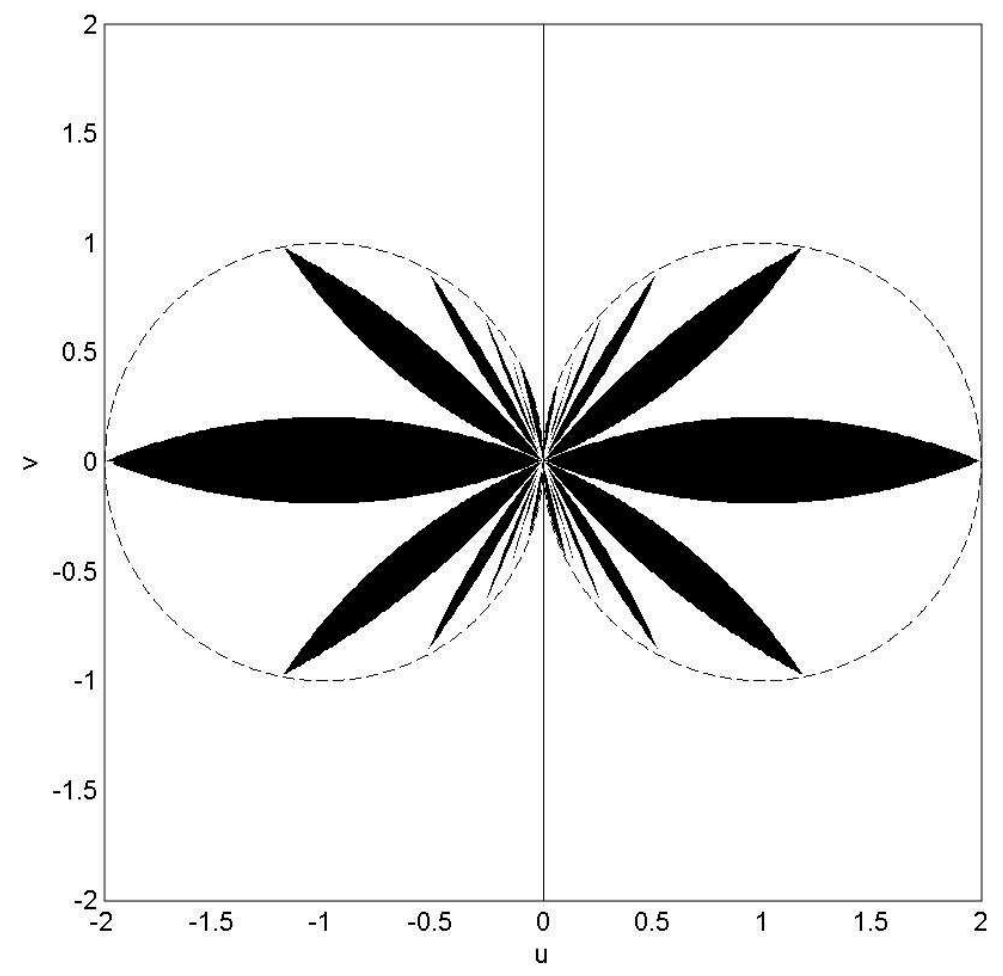

Figure 4.12: Set of $q=u+i v$ for which the system $G(s)=\frac{r}{s^{q}-\left(2048 e^{i \pi}\right)}+\frac{\bar{r}}{s^{\bar{q}}-\left(2048 e^{-i \pi}\right)}$ is stable and causal. 


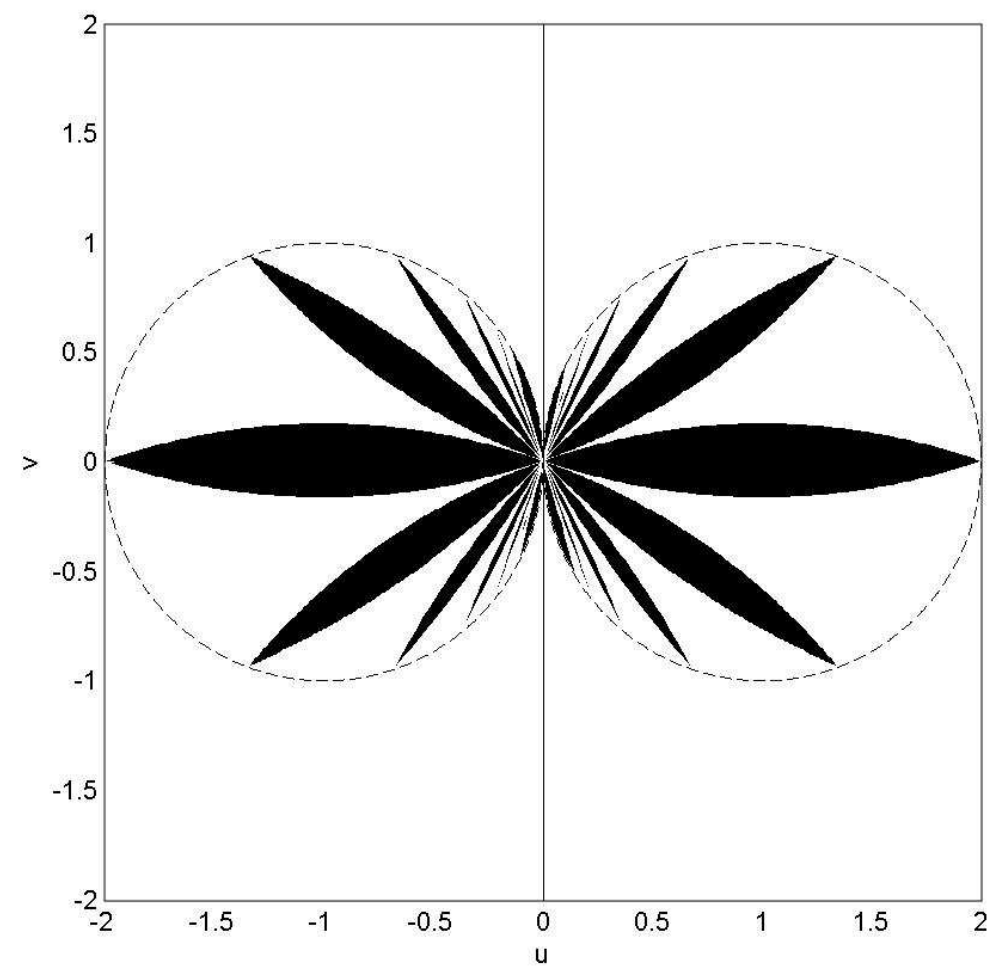

Figure 4.13: Set of $q=u+i v$ for which the system $G(s)=\frac{r}{s^{q}-\left(4096 e^{i \pi}\right)}+\frac{\bar{r}}{s^{\bar{q}}-\left(4096 e^{-i \pi}\right)}$ is stable and causal. 


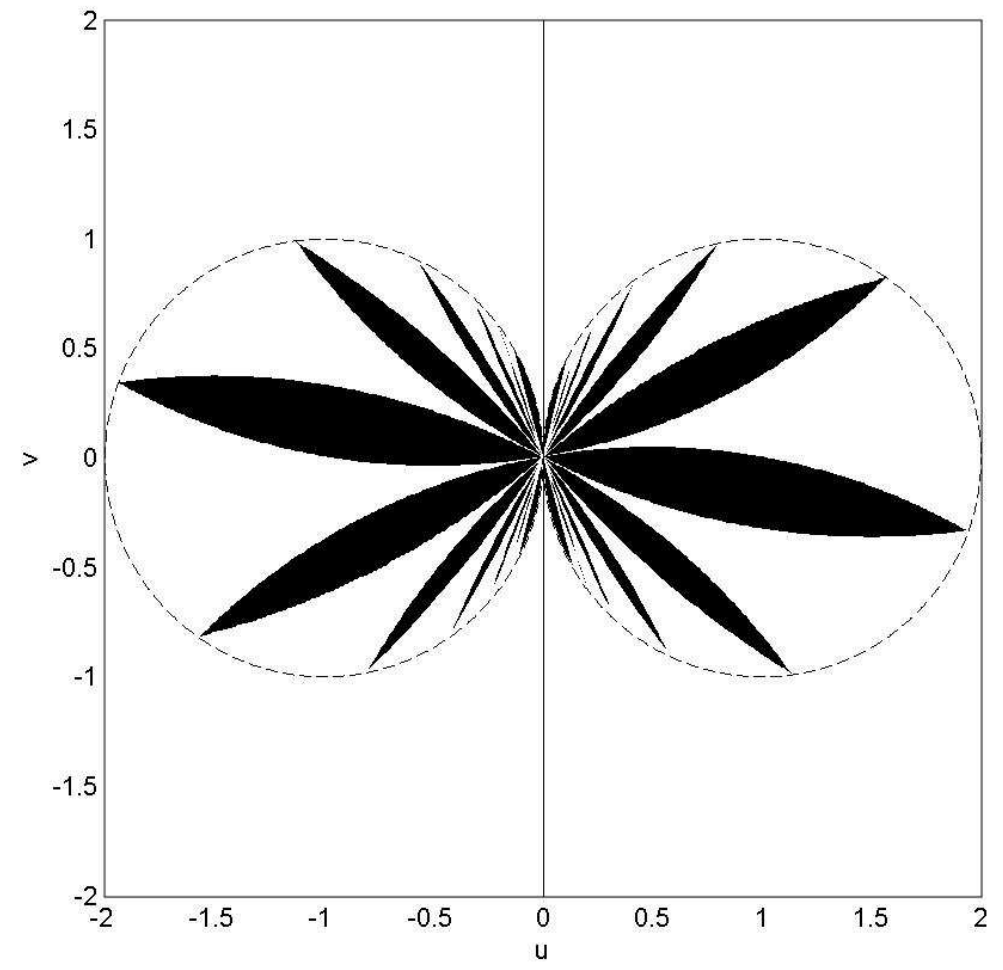

Figure 4.14: Set of $q=u+i v$ for which the system $G(s)=\frac{r}{s^{q}-\left(4096 e^{i \frac{\pi}{2}}\right)}+\frac{\bar{r}}{s^{\bar{q}}-\left(4096 e^{-i \frac{\pi}{2}}\right)}$ is stable and causal. 


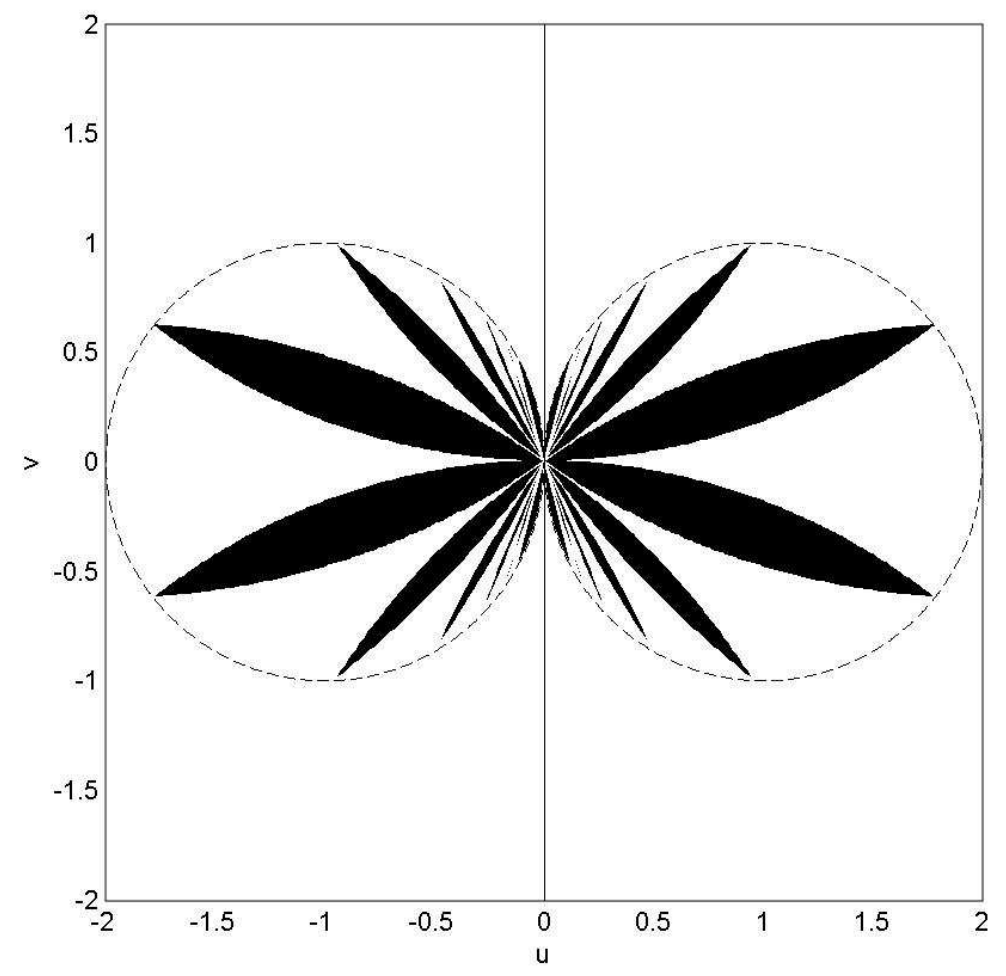

Figure 4.15: Set of $q=u+i v$ for which the system $G(s)=\frac{r}{s^{q}-\left(4096 e^{i 0}\right)}+\frac{\bar{r}}{s^{\bar{q}}-\left(4096 e^{-i 0}\right)}$ is stable and causal. 


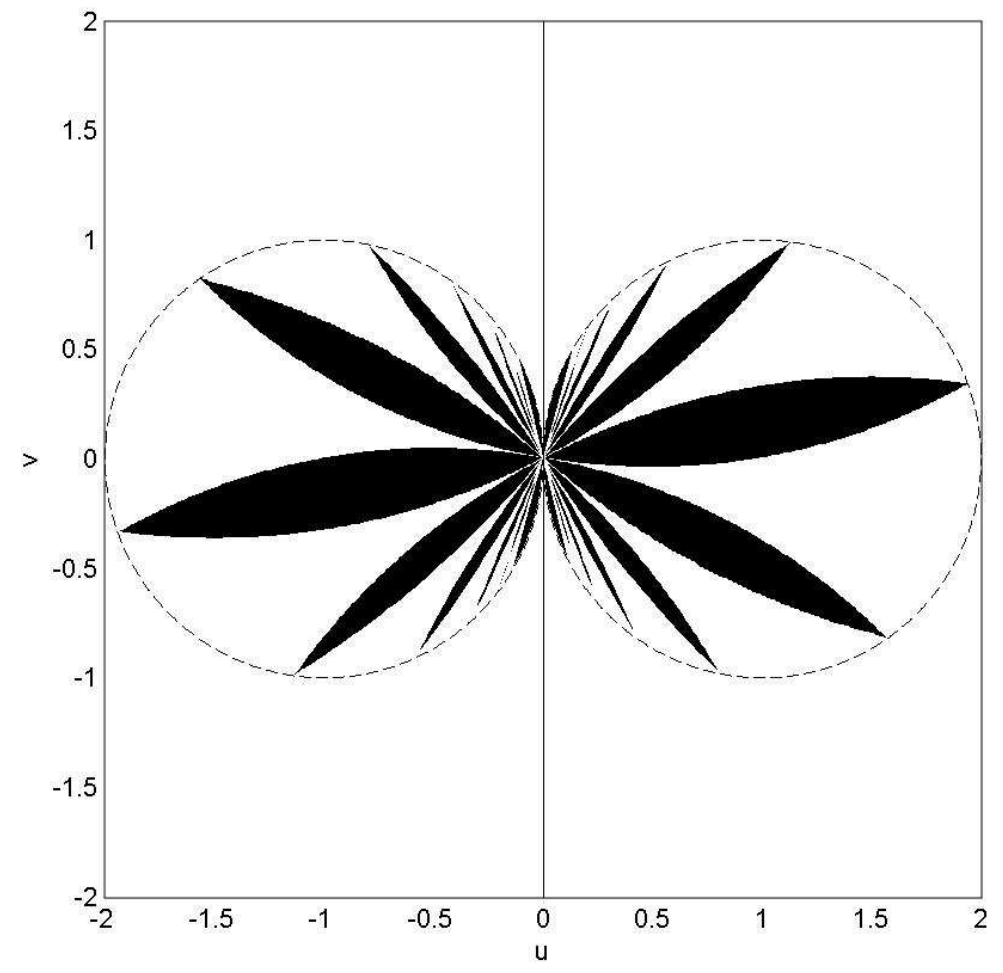

Figure 4.16: Set of $q=u+i v$ for which the system $G(s)=\frac{r}{s^{q}-\left(4096 e^{-i \frac{\pi}{2}}\right)}+\frac{\bar{r}}{s^{\bar{q}}-\left(4096 e^{i \frac{\pi}{2}}\right)}$ is stable and causal. 


\subsubsection{Stable, Causal $G(s)$ for a Fixed $q$}

We now consider for a given $q \in \mathbb{C} \backslash\{0\}$ the set of $a$ for which $G(s)=\frac{r}{s^{q}-a}+\frac{\bar{r}}{s^{q}-\bar{a}}$ corresponds to a stable, causal system. It is easier to consider the set of $a$ for which $G(s)$ corresponds to an unstable or noncausal system. We also want to consider $a$ that are not on the primary Riemann sheet, so we look in the $(\ln |a|, \operatorname{Arg}(a))$ plane rather than the $(\operatorname{Re}(a), \operatorname{Im}(a))$ plane.

Theorem 4.4.5 For $q \in \mathbb{C} \backslash\{0\}$ with $u=\operatorname{Re}(q)$ and $v=\operatorname{Im}(q), G(s)=\frac{r}{s^{q}-a}+\frac{\bar{r}}{s^{\bar{q}}-\bar{a}}$ corresponds to an unstable or noncausal system if for $u=0$

$$
e^{-\frac{\pi}{2}|v|} \leq|a| \leq e^{\frac{\pi}{2}|v|}
$$

or for $\beta=\frac{v}{u}$ with $u \neq 0$

$$
\beta \ln |a|-2 \pi k-\frac{\pi}{2}\left(1+\beta^{2}\right)|u| \leq \operatorname{Arg}(a) \leq \beta \ln |a|-2 \pi k+\frac{\pi}{2}\left(1+\beta^{2}\right)|u|,
$$

any $k \in \mathbb{Z}$ with $u \neq 0$.

Proof Because the poles of $\frac{\bar{r}}{s^{\bar{q}}-\bar{a}}$ are the complex conjugates of $\frac{r}{s^{q}-a}$, we may consider only the poles of $\frac{r}{s^{q}-a}$, which are given by Equation 4.19 as

$$
s_{k}=e^{x \ln |a|+y \operatorname{Arg}(a)+y 2 \pi k} e^{i(x \operatorname{Arg}(a)+x 2 \pi k-y \ln |a|)} .
$$


Then $G(s)$ is either unstable or noncausal if $-\frac{\pi}{2} \leq \operatorname{Arg}\left(s_{k}\right) \leq \frac{\pi}{2}$. This can be written as

$$
-\frac{\pi}{2}\left(u^{2}+v^{2}\right) \leq u \operatorname{Arg}(a)+u 2 \pi k-v \ln |a| \leq \frac{\pi}{2}\left(u^{2}+v^{2}\right)
$$

We consider two cases, where $u=0$ and where $u \neq 0$.

Suppose $u=0$ and $v \neq 0$, then Equation 4.37 becomes

$$
-\frac{\pi}{2} v^{2} \leq v \ln |a| \leq \frac{\pi}{2} v^{2}
$$

If $v>0$, then dividing Equation 4.38 by $v$ gives

$$
-\frac{\pi}{2}|v| \leq \ln |a| \leq \frac{\pi}{2}|v|
$$

If $v<0$, then dividing Equation 4.38 by $v$ gives

$$
\frac{\pi}{2}|v| \geq \ln |a| \geq-\frac{\pi}{2}|v|
$$

which is the same as Equation 4.39. Hence, $|a|$ satisfies

$$
e^{-\frac{\pi}{2}|v|} \leq|a| \leq e^{\frac{\pi}{2}|v|}
$$

which is a vertical strip in the $(|a|, \operatorname{Arg}(a))$ plane. 
Suppose now that $u \neq 0$ and $v=\beta v$. Then Equation 4.37 can be written as

$$
-\frac{\pi}{2}\left(1+\beta^{2} u^{2} \leq u \operatorname{Arg}(a)+u 2 \pi k-\beta u \ln |a| \leq \frac{\pi}{2}\left(1+\beta^{2}\right) u^{2} .\right.
$$

If $u>0$, then dividing Equation 4.40 by $u$ gives

$$
-\frac{\pi}{2}\left(1+\beta^{2}|u| \leq \operatorname{Arg}(a)+2 \pi k-\beta \ln |a| \leq \frac{\pi}{2}\left(1+\beta^{2}\right)|u| .\right.
$$

Similarly if $u<0$, then dividing Equation 4.40 by $u$ gives

$$
\frac{\pi}{2}\left(1+\beta^{2}|u| \geq \operatorname{Arg}(a)+2 \pi k-\beta \ln |a| \geq-\frac{\pi}{2}\left(1+\beta^{2}\right)|u|,\right.
$$

which is the same as Equation 4.41. Isolating $\operatorname{Arg}(a)$ in Equation 4.41 gives

$$
\beta \ln |a|-2 \pi k-\frac{\pi}{2}\left(1+\beta^{2}\right)|u| \leq \operatorname{Arg}(a) \leq \beta \ln |a|-2 \pi k+\frac{\pi}{2}\left(1+\beta^{2}\right)|u|,
$$

which is a curved strip in the $(|a|, \operatorname{Arg}(a))$ plane for each $k \in \mathbb{Z}$.

Note that if $\beta=0$, which means that $q \in \mathbb{R}$, then Equation 4.42 reduces to

$$
-\frac{\pi}{2}|u| \leq \operatorname{Arg}(a) \leq \frac{\pi}{2}|u|
$$


which is the result of [56]. We can now consider for which values of $a$ the transfer function $G(s)$ corresponds to a stable, causal system.

Corollary 4.4.6 For $q \in \mathbb{C} \backslash\{0\}$ with $u=\operatorname{Re}(q)$ and $v=\operatorname{Im}(q), G(s)=\frac{r}{s^{q}-a}+\frac{\bar{r}}{s^{\bar{q}}-\bar{a}}$ corresponds to a stable, causal system if

$$
\begin{aligned}
& \qquad|a| \notin\left[e^{-\frac{\pi}{2}|v|}, e^{\frac{\pi}{2}|v|}\right], \\
& \text { for } u=0 \text { or } \\
& \beta \ln |a|-2 \pi(k+1)-\frac{\pi}{2}\left(1+\beta^{2}\right)|u| \leq \operatorname{Arg}(a) \leq \beta \ln |a|-2 \pi k+\frac{\pi}{2}\left(1+\beta^{2}\right)|u|,
\end{aligned}
$$

for any $k \in \mathbb{Z}$ with $u \neq 0$.

Proof We take the complement of the sets from Theorem 4.4.5.

\subsubsection{Examples}

Now we apply Corollary 4.4.6 to a variety of $q=u+i v$ such that $(u-1)^{2}+v^{2}<1$. Figures 4.17 through 4.23 show the effect on the set of stable $a$ when $\beta$ is held constant at unity and $u$ is increased from $\frac{1}{10}$ to $\frac{99}{100}$. The band of stable $a$ decreases as $u$ increases, while the center of the bands are close to each other. Figure 4.23 is close to the case when $u=1$ and $\beta=1$. Figures 4.24 through 4.28 show the effects 
on the set of stable $a$ when $u$ is held constant at unity and beta is decreased from $\frac{4}{5}$ through 0. Figure 4.28 is the integer-order case, whose results are as expected.

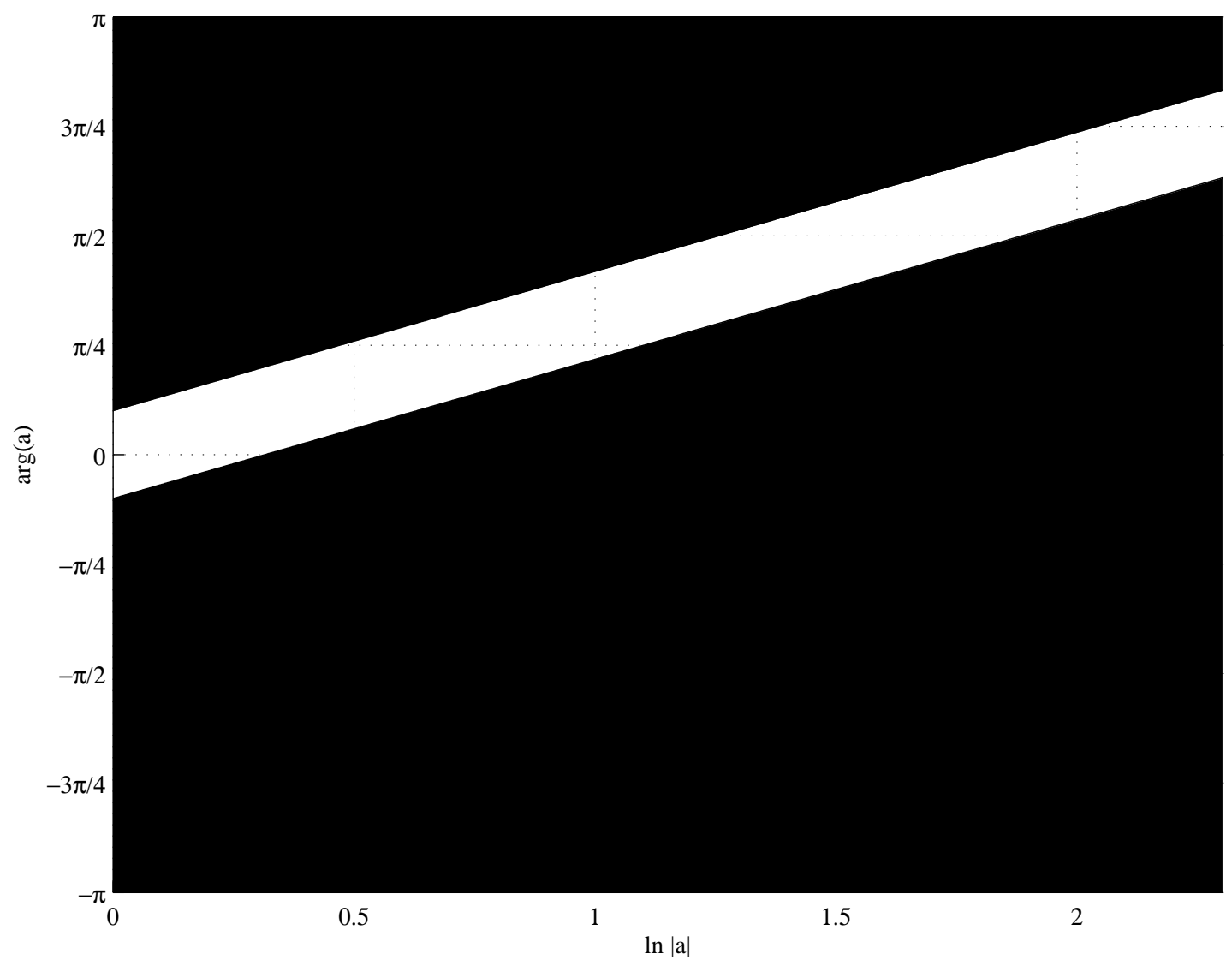

Figure 4.17: Set of $a=|a| e^{i \operatorname{Arg}(a)}$ for which the system $G(s)=\frac{r}{s^{\frac{1}{10}(1+i)}-a}+\frac{\bar{r}}{s^{\frac{1}{10}(1-i)}-\bar{a}}$ is stable and causal. 


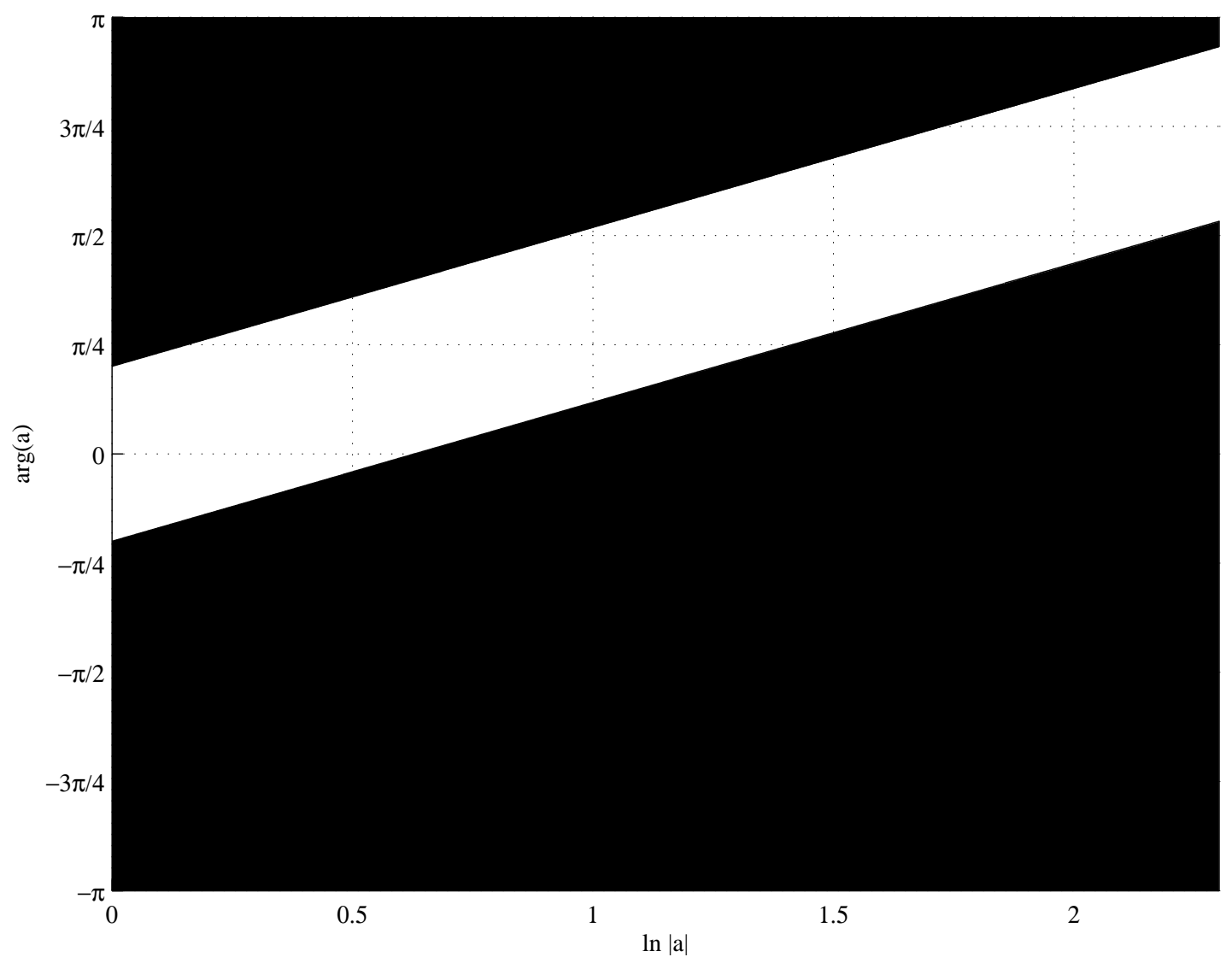

Figure 4.18: Set of $a=|a| e^{i \operatorname{Arg}(a)}$ for which the system $G(s)=\frac{r}{s^{\frac{1}{5}(1+i)}-a}+\frac{\bar{r}}{s^{\frac{1}{5}(1-i)}-\bar{a}}$ is stable and causal. 


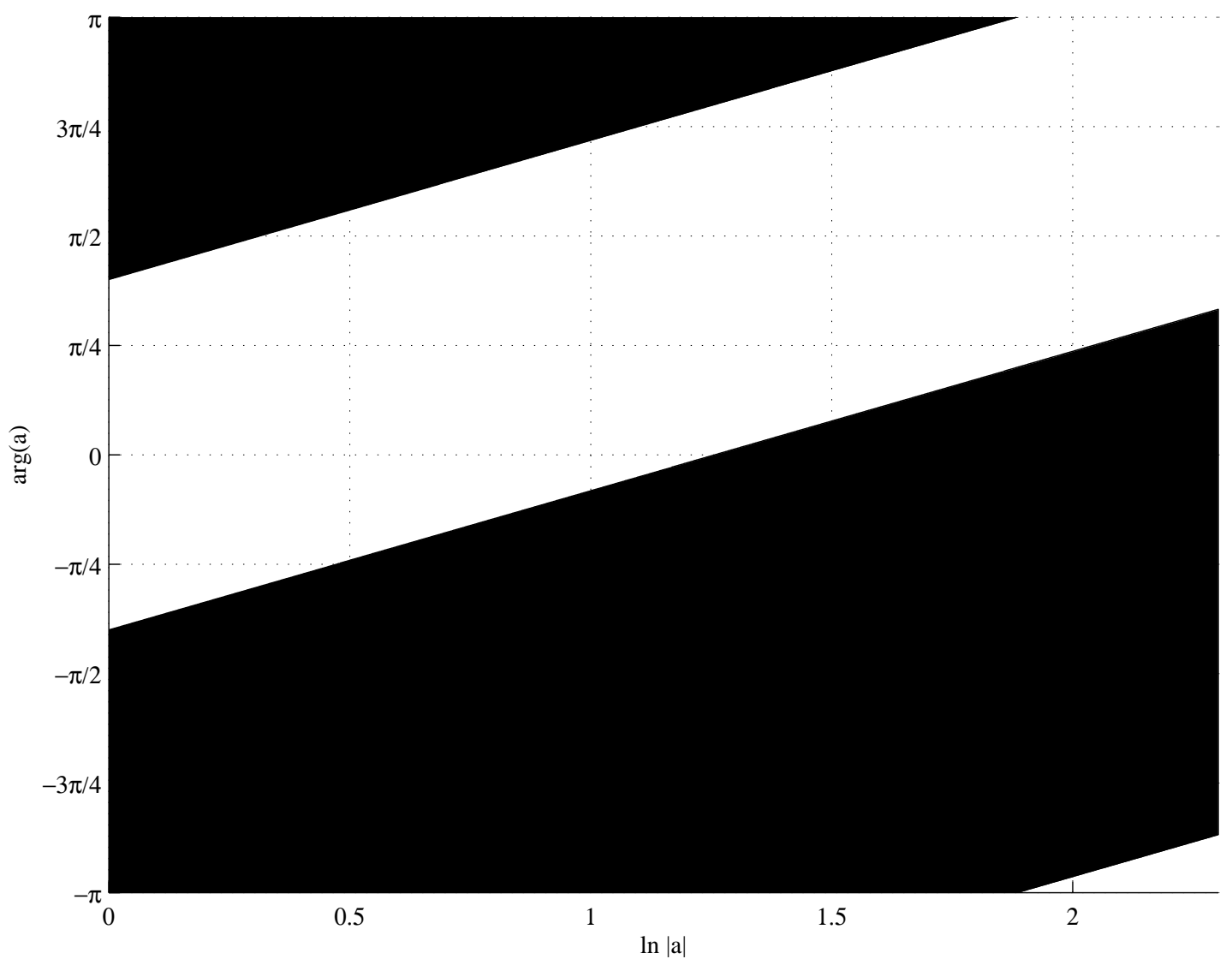

Figure 4.19: Set of $a=|a| e^{i \operatorname{Arg}(a)}$ for which the system $G(s)=\frac{r}{s^{\frac{2}{5}(1+i)}-a}+\frac{\bar{r}}{s^{\frac{2}{5}(1-i)}-\bar{a}}$ is stable and causal. 


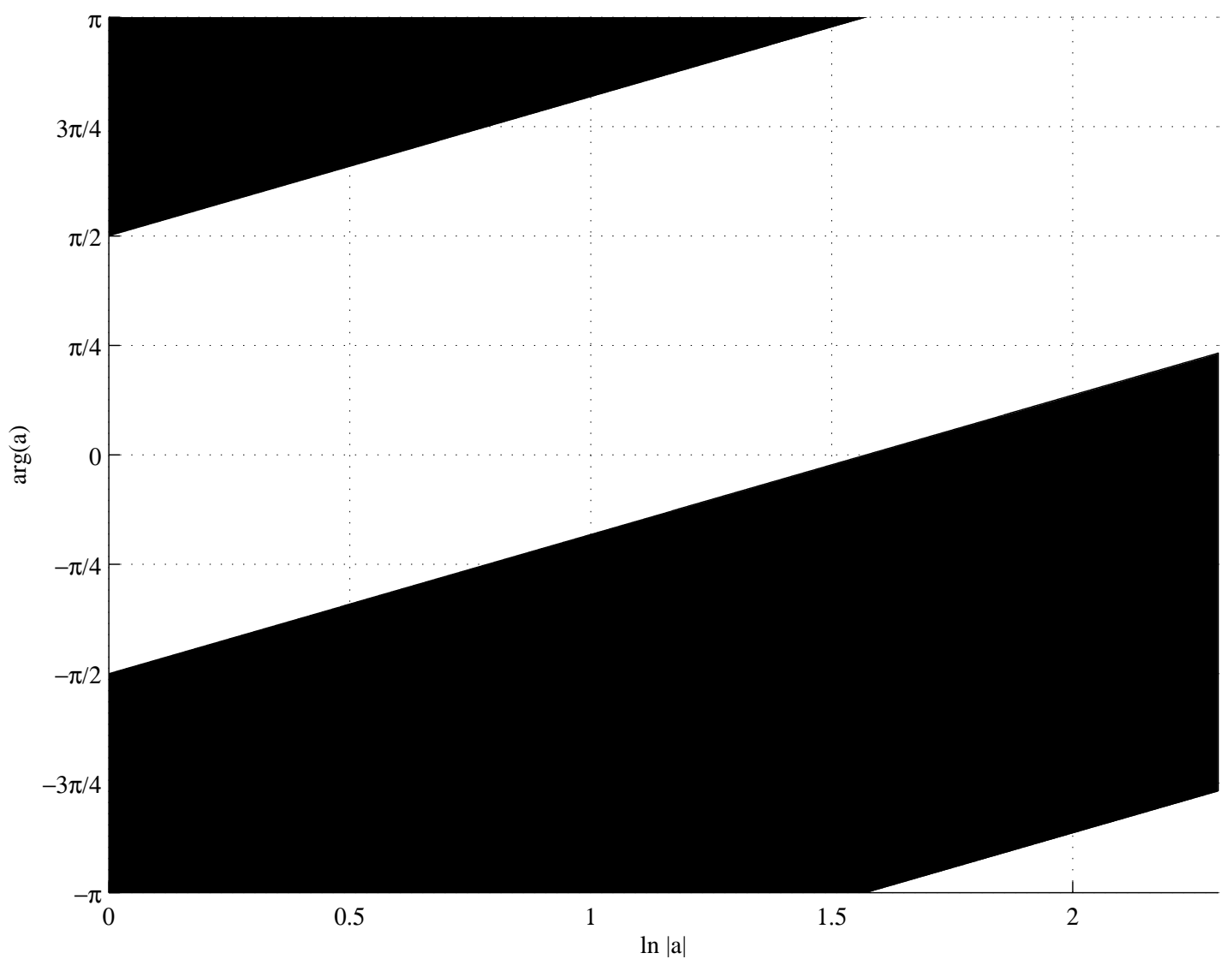

Figure 4.20: Set of $a=|a| e^{i \operatorname{Arg}(a)}$ for which the system $G(s)=\frac{r}{s^{\frac{1}{2}(1+i)}-a}+\frac{\bar{r}}{s^{\frac{1}{2}(1-i)}-\bar{a}}$ is stable and causal. 


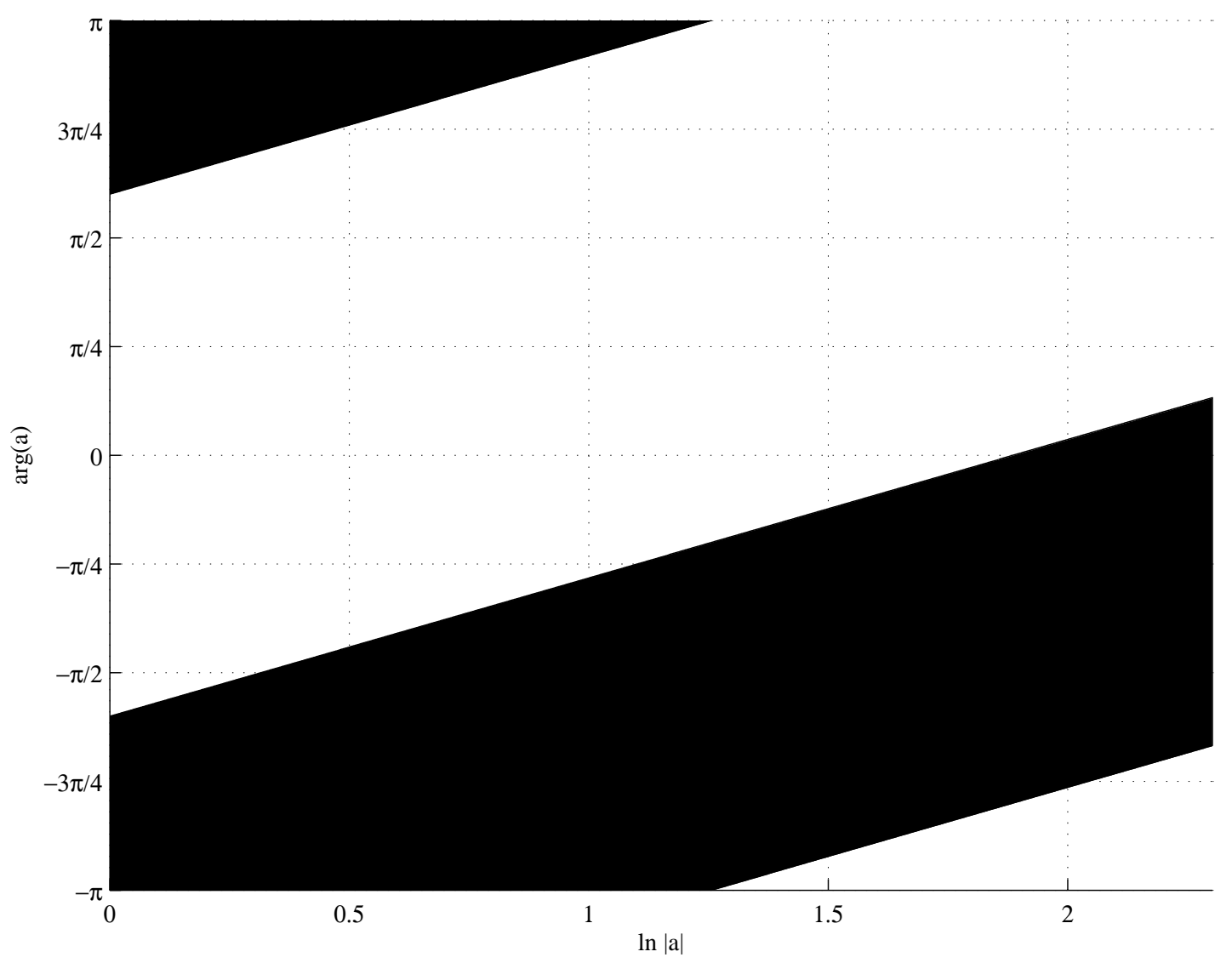

Figure 4.21: Set of $a=|a| e^{i \operatorname{Arg}(a)}$ for which the system $G(s)=\frac{r}{s^{\frac{3}{5}(1+i)}-a}+\frac{\bar{r}}{s^{\frac{3}{5}(1-i)}-\bar{a}}$ is stable and causal.

94 


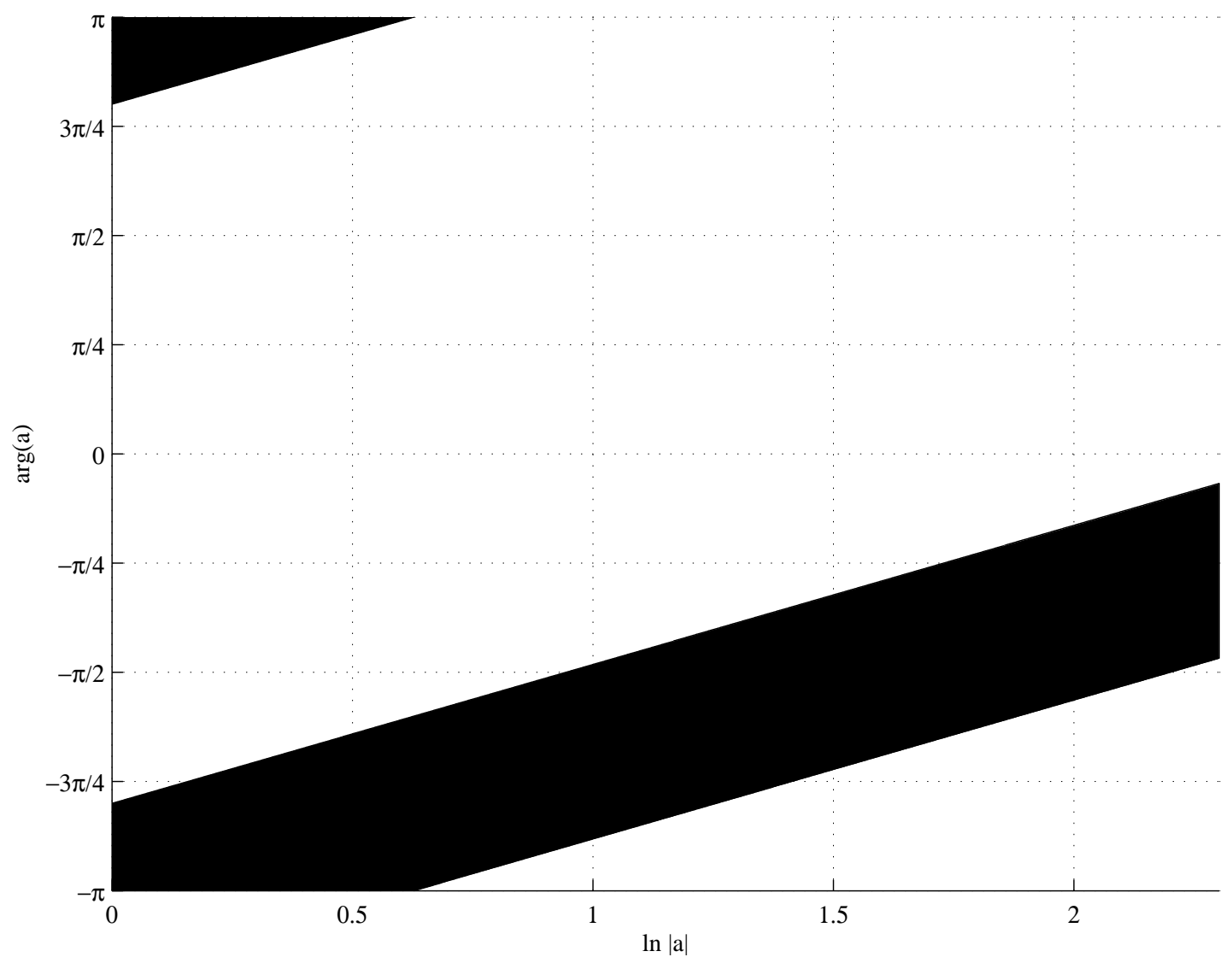

Figure 4.22: Set of $a=|a| e^{i \operatorname{Arg}(a)}$ for which the system $G(s)=\frac{r}{s^{\frac{4}{5}(1+i)}-a}+\frac{\bar{r}}{s^{\frac{4}{5}(1-i)}-\bar{a}}$ is stable and causal. 


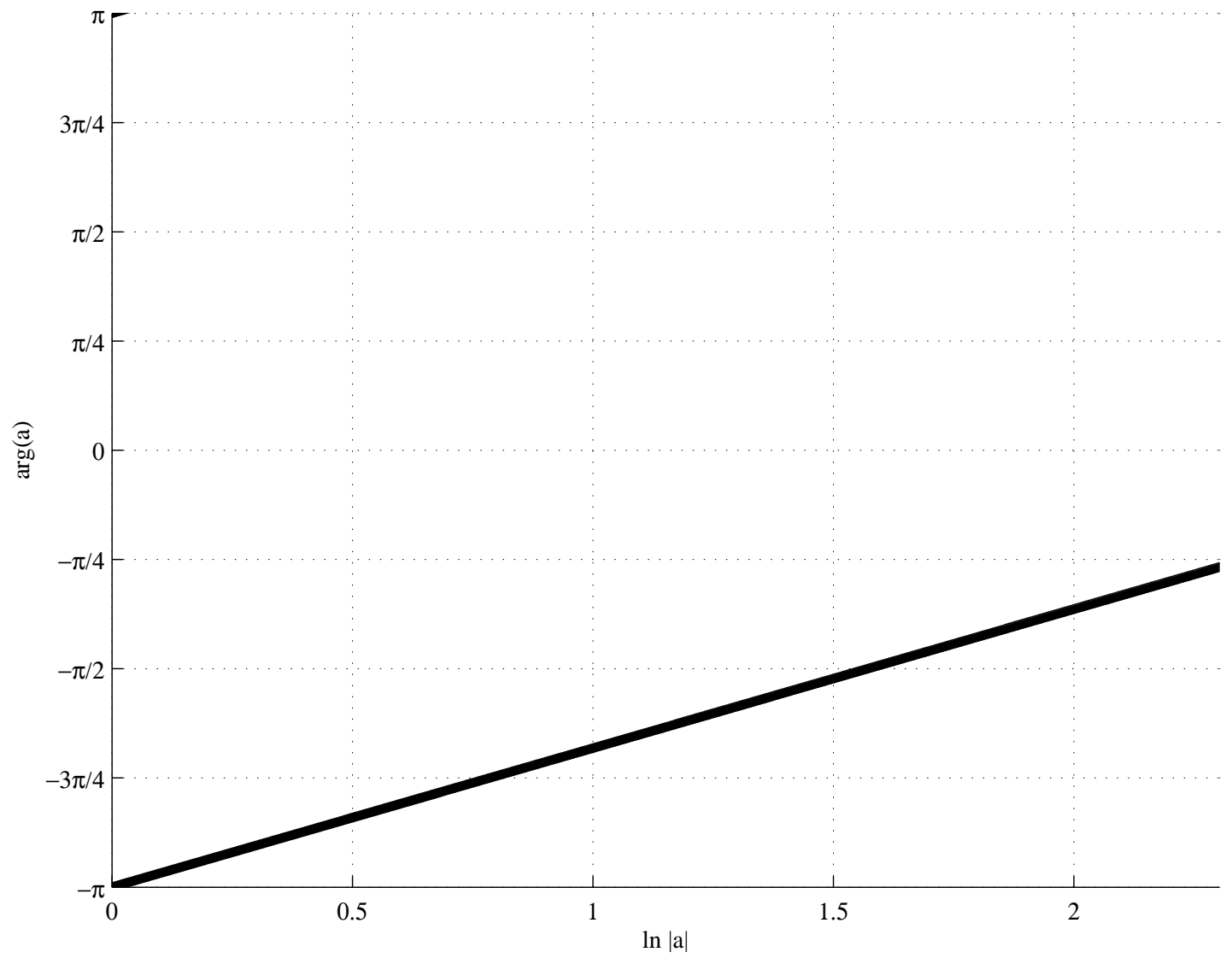

Figure 4.23: Set of $a=|a| e^{i \operatorname{Arg}(a)}$ for which the system $G(s)=\frac{r}{s^{0.99(1+i)}-a}+\frac{\bar{r}}{s^{0.99(1-i)}-\bar{a}}$ is stable and causal. 


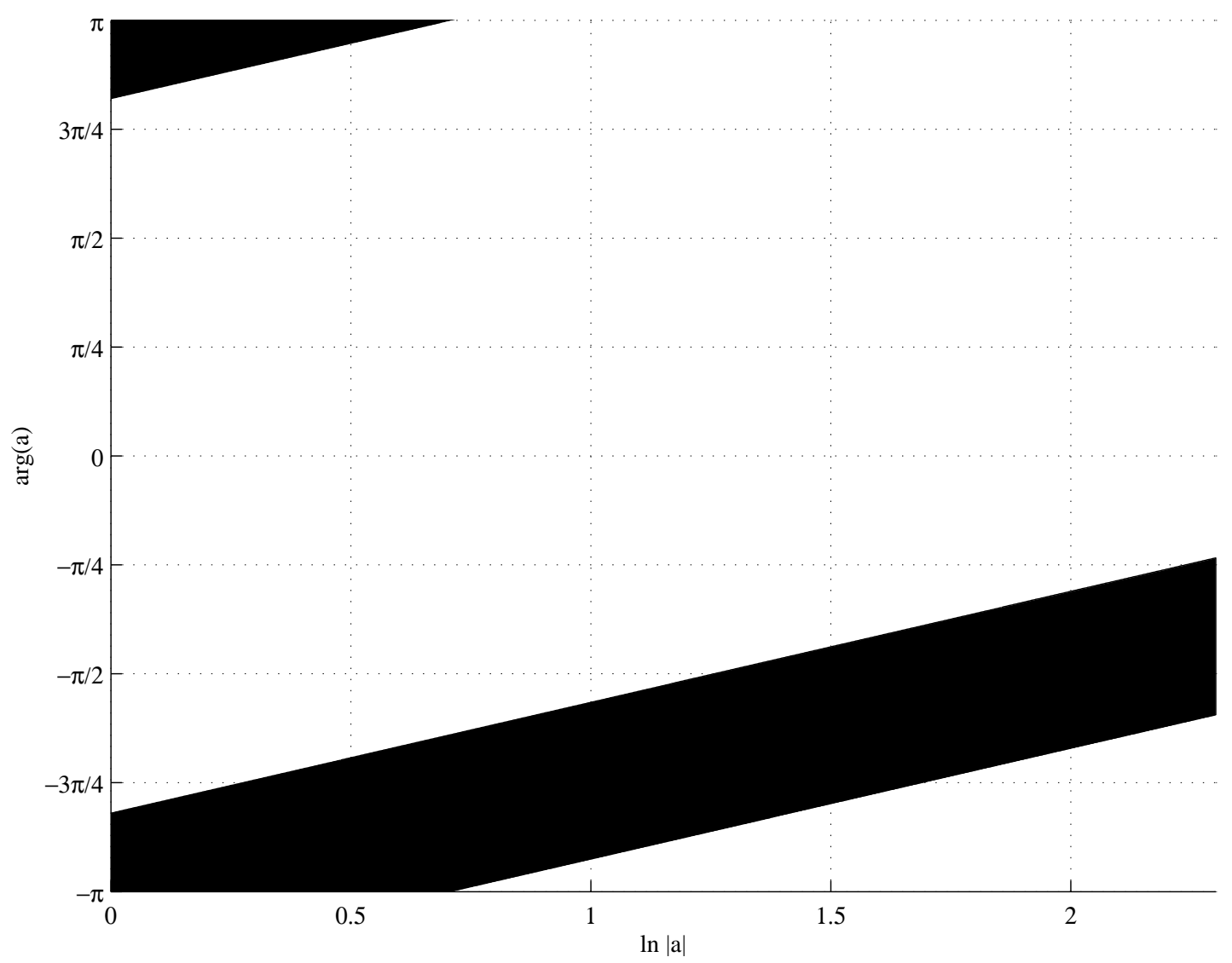

Figure 4.24: Set of $a=|a| e^{i \operatorname{Arg}(a)}$ for which the system $G(s)=\frac{r}{s^{\left(1+\frac{4 i}{5}\right)}-a}+\frac{\bar{r}}{s^{\left(1-\frac{4 i}{5}\right)}-\bar{a}}$ is stable and causal. 


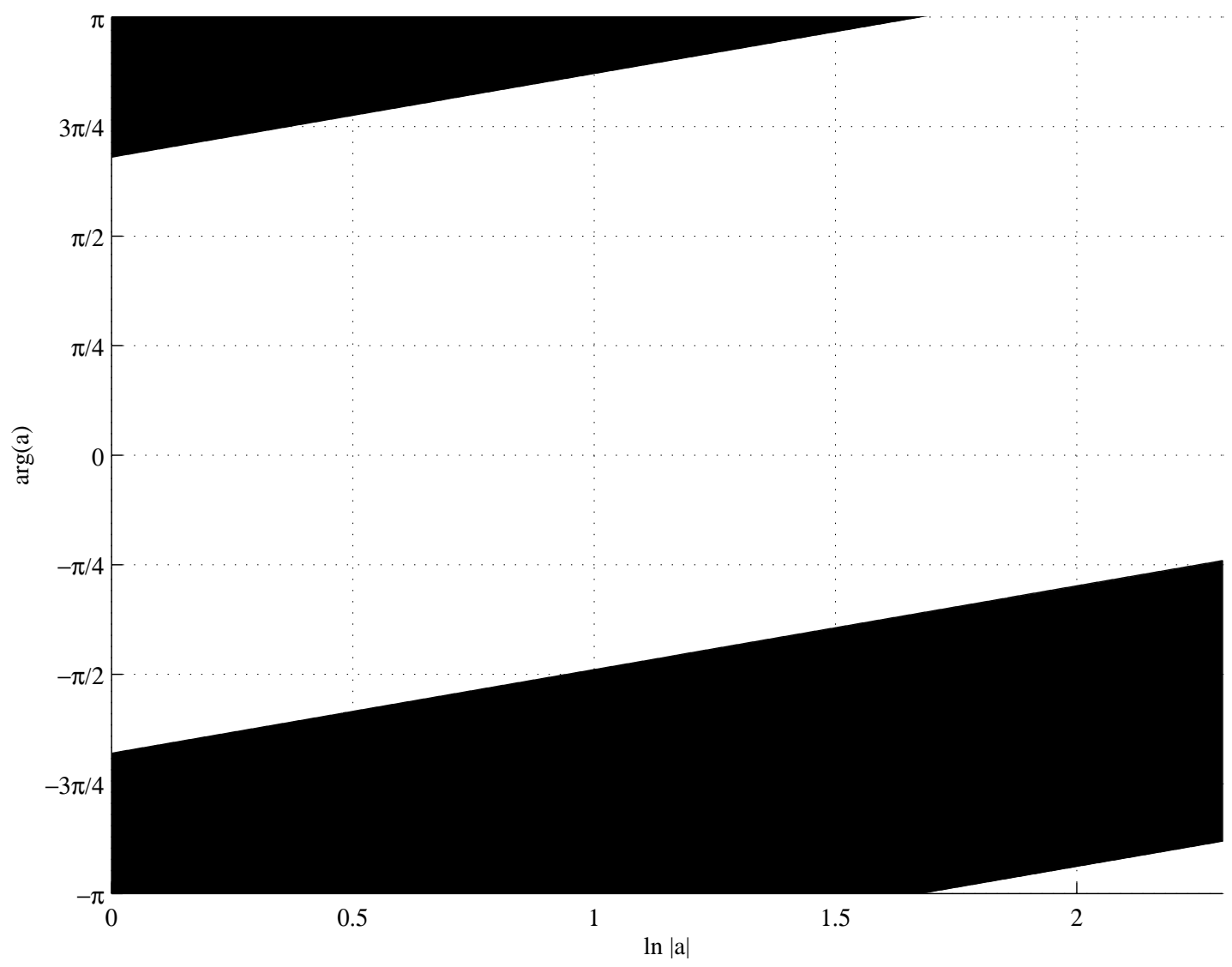

Figure 4.25: Set of $a=|a| e^{i \operatorname{Arg}(a)}$ for which the system $G(s)=\frac{r}{s^{\left(1+\frac{3 i}{5}\right)}-a}+\frac{\bar{r}}{s^{\left(1-\frac{3 i}{5}\right)}-\bar{a}}$ is stable and causal.

98 


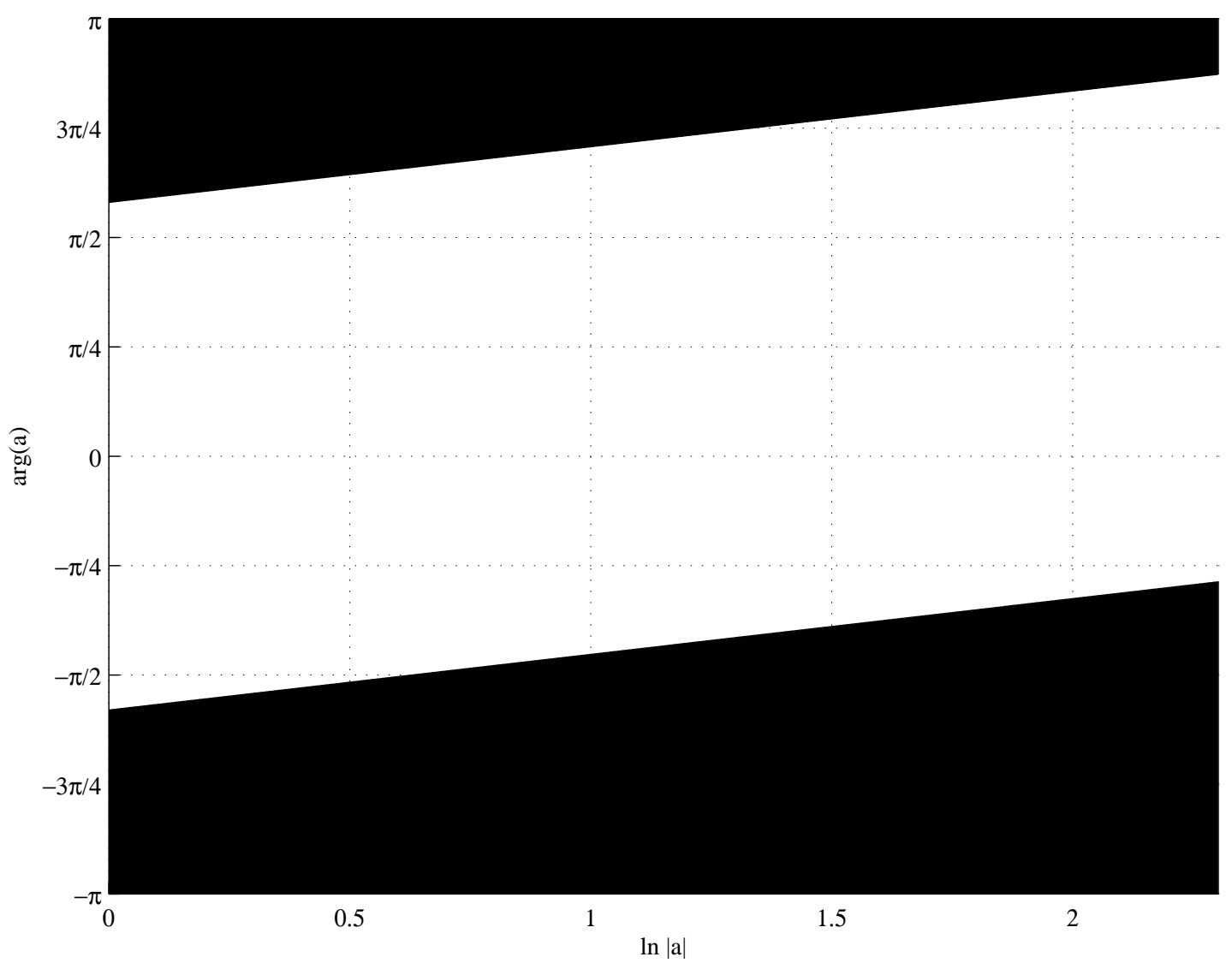

Figure 4.26: Set of $a=|a| e^{i \operatorname{Arg}(a)}$ for which the system $G(s)=\frac{r}{s^{\left(1+\frac{2 i}{5}\right)}-a}+\frac{\bar{r}}{s^{\left(1-\frac{2 i}{5}\right)}-\bar{a}}$ is stable and causal. 


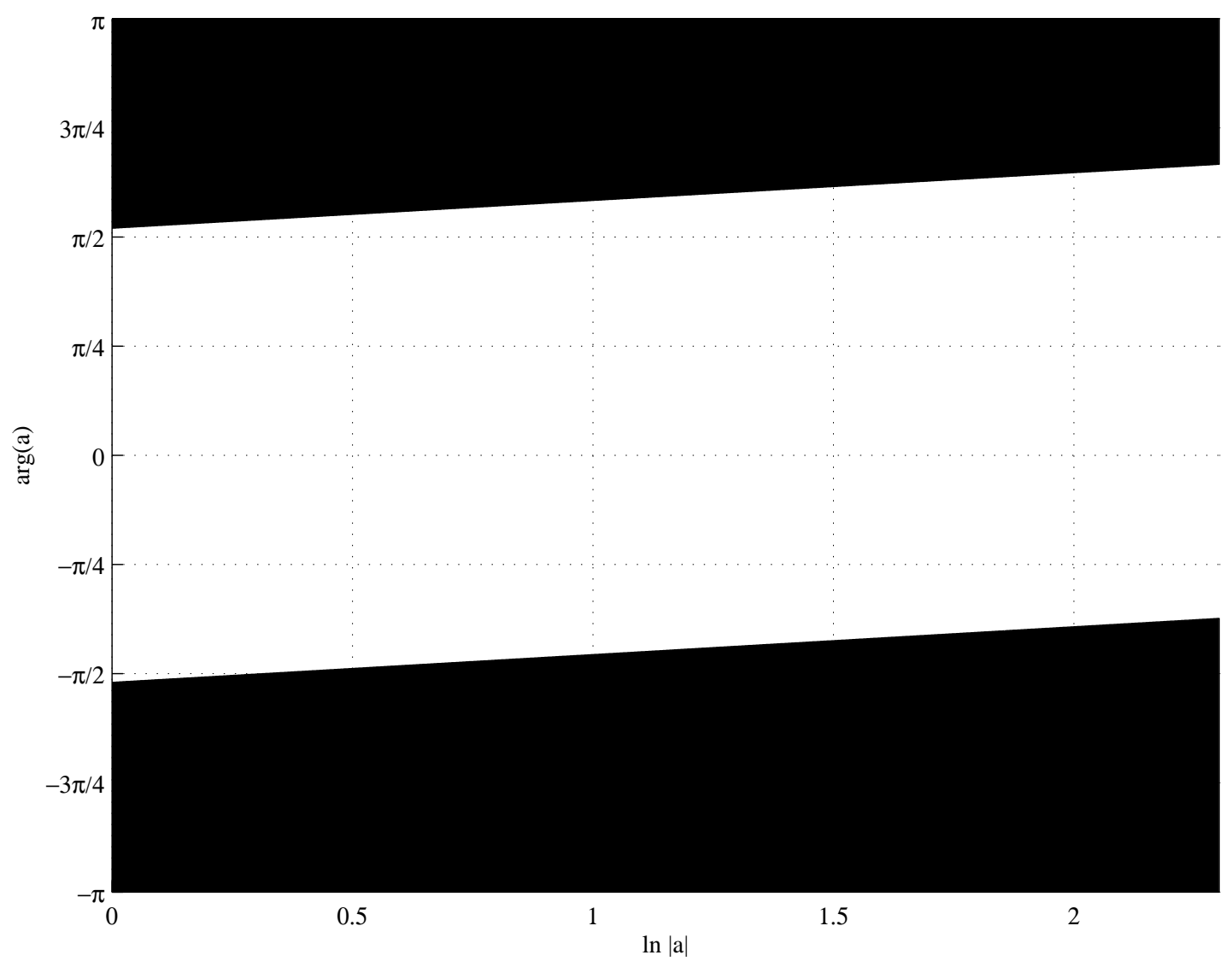

Figure 4.27: Set of $a=|a| e^{i \operatorname{Arg}(a)}$ for which the system $G(s)=\frac{r}{s^{\left(1+\frac{i}{5}\right)}-a}+\frac{\bar{r}}{s^{\left(1-\frac{i}{5}\right)}-\bar{a}}$ is stable and causal. 


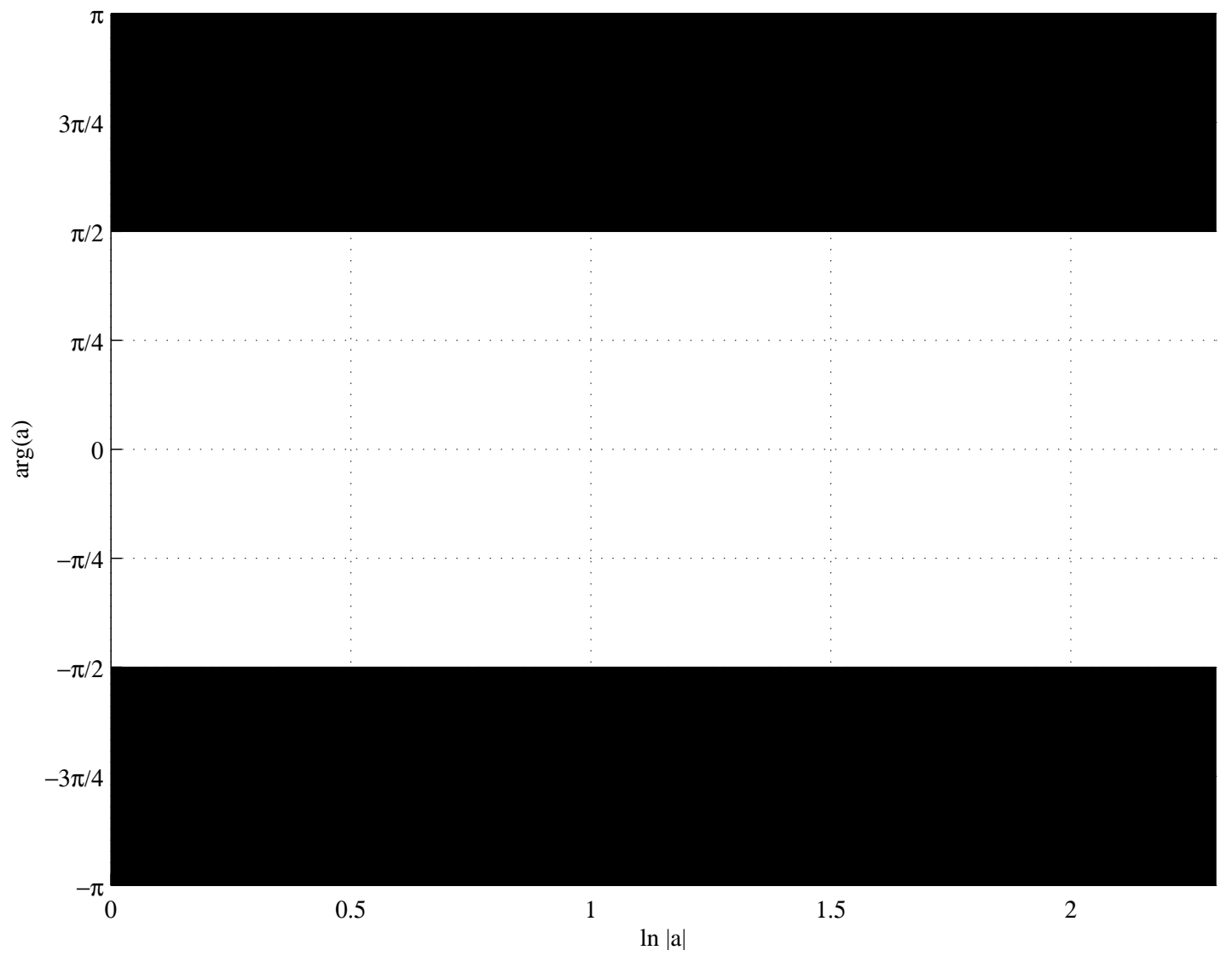

Figure 4.28: Set of $a=|a| e^{i \operatorname{Arg}(a)}$ for which the system $G(s)=\frac{r}{s^{1}-a}+\frac{\bar{r}}{s^{1}-\bar{a}}$ is stable and causal. 


\subsection{Conclusions}

In this chapter it has been demonstrated that pairs of complex-conjugate complexorder differintegrals map real-valued functions to real-valued functions. The funda-

mental, linear conjugate-order system $G(s)=\frac{r}{s^{q}-a}+\frac{\bar{r}}{s^{\bar{q}}-\bar{a}}$ has been introduced. $G(s)$ maps real-valued functions to real-valued functions. The poles of $G(s)$ have been determined, and the sets of $q$ and $a$ for which $G(s)$ corresponds to a stable, causal system have been found. Numerous examples of such $q$ for a fixed $a$ and of such $a$ for a fixed $q$ have been given. Next, we discuss Hilbert spaces as an abstract setting in which we may consider fractional-order operators. 


\section{CHAPTER V}

\section{OPERATORS ON A HILBERT SPACE}

We have demonstrated in Chapter 2 that integer-order, linear, time-invariant systems can be represented as convolution operators. In this chapter we provide a general and abstract framework in which convolution operators may be considered. To that end Hilbert spaces are introduced. Compact operators, those operators to which linear operators on finite-dimensional spaces can be generalized, are discussed. The most important result concerning compact operators is the Schmidt decomposition, which indicates that a compact operator can be fully described by a countable set of scalars, called singular values, and a countable set of basis-vector pairs for the domain and range of the operator, called Schmidt pairs. We then focus on the Hilbert space $L_{2}(\mathbb{R})$ and some isomorphisms of $L_{2}(\mathbb{R})$, specifically $L_{2}(i \mathbb{R}), L_{2}(\mathbb{T})$, and $\ell_{2}(\mathbb{Z})$. Boundedness, compactness, and finite-rank criteria for integral operators on $L_{2}(\mathbb{R})$ are considered. This chapter closes with three methods by which the eigenvalues, and thus the singular values, of compact Hermitian operators may be evaluated. The first of these methods will be applied to the Hankel operators of fractional-order systems in Chapter 7. 


\subsection{Hilbert Spaces}

We begin by defining norms and inner products, which allow us to define the geometry of the Hilbert space. We will let $\mathbb{F}$ denote either $\mathbb{C}$ or $\mathbb{R}$.

Definition Let $\mathcal{X}$ be a vector space over a field $\mathbb{F}$. A norm on $\mathcal{X}$ is a function $\|\cdot\|: \mathcal{X} \rightarrow \mathbb{R}$ such that for $\alpha \in \mathbb{F}$ and for $x, y \in \mathcal{X}$

1. $\|\alpha x\|=|\alpha|\|x\|$ and

2. $\|x+y\| \leq\|x\|+\|y\|$.

An inner product on $\mathcal{X}$ is a function $\langle\cdot, \cdot\rangle: \mathcal{X} \times \mathcal{X} \rightarrow \mathbb{F}$ such that for all $\alpha, \beta \in \mathbb{F}$ and for all $x, y, z \in \mathcal{X}$

1. $\langle x, y\rangle=\overline{\langle y, x\rangle}$

2. $\langle\alpha x+\beta y, z\rangle=\alpha\langle x, z\rangle+\beta\langle y, z\rangle$

3. $\langle x, x\rangle \in \mathbb{R}$ and $\langle x, x\rangle \geq 0$

4. $\langle x, x\rangle=0$ if and only if $x=0$.

For clarity, the space over which a norm or inner product is taken may be indicated by a subscript, for example $\|\cdot\|_{\mathcal{X}}$ or $\langle\cdot, \cdot\rangle_{\mathcal{X}}$. When it is clear which space is associated with the norm or inner product, the subscript will be omitted.

For any inner product $\langle\cdot, \cdot\rangle,\|x\|=(\langle x, x\rangle)^{\frac{1}{2}}$ is a norm. This norm is called the norm induced by the inner product. With the definitions of a norm and an inner product, a Hilbert space may be defined. 
Definition A Hilbert space is a vector space $\mathcal{H}$ over a field $\mathbb{F}$ along with an inner product $\langle\cdot, \cdot\rangle$ such that, relative to the norm induced by the inner product, every Cauchy sequence in $\mathcal{H}$ converges to an element of $\mathcal{H}$. That is, a Hilbert space is a complete inner-product space.

Theorem 5.1.1 Let $E \subset \mathbb{R}^{n}$ be measurable. $L_{2}(E)$, the space of all functions squareintegrable on $E$, is a Hilbert space with $\langle f, g\rangle=\int_{E} f \bar{g}$ for $f, g \in L_{2}(E)$.

Proof See [20].

Subsequently, we will use the fact that $\mathbb{R}, \mathbb{R}_{+}, \mathbb{R}_{-}, i \mathbb{R}$, and $\mathbb{T}$ are measurable to discuss operators on the Hilbert spaces $L_{2}(\mathbb{R}), L_{2}\left(\mathbb{R}_{+}\right), L_{2}\left(\mathbb{R}_{-}\right), L_{2}(i \mathbb{R})$, and $L_{2}(\mathbb{T})$

One of the most useful concepts of a Hilbert space is the idea of orthogonality. Orthogonality describes the underlying geometric structure of a Hilbert space.

Definition Let $\mathcal{H}$ be a Hilbert space and let $f, g \in \mathcal{H} . \quad f$ and $g$ are said to be orthogonal if $\langle f, g\rangle=0 .\left\{f_{k}\right\}$ is said to be an orthogonal set if $\left\langle f_{m}, f_{n}\right\rangle=0$ whenever $m \neq n .\left\{f_{k}\right\}$ is said to be an orthonormal set if $\left\{f_{k}\right\}$ is an orthogonal set and if each member of $\left\{f_{k}\right\}$ has unity norm. A basis is a maximal orthonormal set.

One use of orthogonality is in the idea of an orthogonal complement, which will be used to decompose spaces into two orthogonal subspaces.

Definition Let $\mathcal{H}$ be a Hilbert space and let $E$ be a subspace of $\mathcal{H}$. The orthogonal complement of $E$ is the set $E^{\perp}=\{g \in \mathcal{H}:\langle g, f\rangle=0$ for all $f \in E\}$. 
The direct sum is a way of decomposing a given Hilbert space into component spaces, or building a larger Hilbert space from Hilbert spaces.

Definition Let $\mathcal{M}$ and $\mathcal{N}$ be subspaces of the Hilbert space $\mathcal{H}$. $\mathcal{H}$ is the direct sum of $\mathcal{M}$ and $\mathcal{N}$, denoted $\mathcal{H}=\mathcal{M} \oplus \mathcal{N}$, if $\mathcal{M} \cap \mathcal{N}=\{0\}$ and if every member of $\mathcal{H}$ can be expressed as the sum of a member of $\mathcal{M}$ with a member of $\mathcal{N}$. If $\langle m, n\rangle=0$ for all $m \in \mathcal{M}$ and for all $n \in \mathcal{N}$, then $\mathcal{H}$ is said to be the orthogonal direct sum of $\mathcal{M}$ and $\mathcal{N}[87]$

It is clear that if $\mathcal{H}$ is the orthogonal direct sum of $\mathcal{M}$ and $\mathcal{N}$, then $\mathcal{N}=\mathcal{M}^{\perp}$. The converse is also true.

Theorem 5.1.2 If $\mathcal{M}$ is a subspace of a Hilbert space $\mathcal{H}$, then $\mathcal{H}=\mathcal{M} \oplus \mathcal{M}^{\perp}$; that is, $\mathcal{H}$ is the orthogonal direct sum of $\mathcal{M}$ and $\mathcal{M}^{\perp}$.

Proof See [20].

We use this result in Section 5.5 to obtain a decomposition of $L_{2}(\mathbb{T})$.

We next define a map that preserves the basic structure of the Hilbert space.

Definition Let $\mathcal{H}$ and $\mathcal{N}$ be Hilbert spaces. An isomorphism between $\mathcal{H}$ and $\mathcal{N}$ is a linear surjection $U: \mathcal{H} \rightarrow \mathcal{N}$ such that

$$
\langle U h, U g\rangle_{\mathcal{N}}=\langle h, g\rangle_{\mathcal{H}}
$$

for all $g, h \in \mathcal{H}$. $\mathcal{H}$ and $\mathcal{N}$ are said to be isomorphic, which is denoted $\mathcal{H} \cong \mathcal{N}$. 
It can be shown that being isomorphic is an equivalence relation for Hilbert spaces [20]. This is justified because the inner product describes the essential relationship between members of the Hilbert space. Since the relationship between two members of $\mathcal{H}$ is the same as that between the corresponding members of $\mathcal{N}, \mathcal{H}$ and $\mathcal{N}$ can be considered the same as each other.

\subsection{Bounded Operators}

We now discuss linear operators on a Hilbert space, paying particular attention to bounded operators which play an important role in system theory because, on $L_{2}(\mathbb{R})$, a bounded operator corresponds with a stable system.

Definition Let $\mathcal{H}$ and $\mathcal{N}$ be Hilbert spaces and let $K: \mathcal{H} \rightarrow \mathcal{N}$ be a linear operator. The norm of $K$, denoted $\|K\|$, is defined by

$$
\|K\|=\sup \left\{\|K h\|_{\mathcal{N}}: h \in \mathcal{H},\|h\|_{\mathcal{H}} \leq 1\right\} .
$$

If $\|K\|<\infty$, then $K$ is said to be bounded.

Definition $\mathcal{B}(\mathcal{H}, \mathcal{N})$ is the set of all linear bounded operators from $\mathcal{H}$ to $\mathcal{N} \cdot \mathcal{B}(\mathcal{H})$ is the set of all linear bounded operators from $\mathcal{H}$ to $\mathcal{H}$.

There are equivalent definitions of the norm of an operator, given by the following theorem. 
Theorem 5.2.1 Let $\mathcal{H}$ and $\mathcal{N}$ be Hilbert spaces, and let $K: \mathcal{H} \rightarrow \mathcal{N}$ be a linear operator. Then

$$
\begin{aligned}
\|K\| & =\sup \left\{\|K h\|_{\mathcal{N}}: h \in \mathcal{H},\|h\|_{\mathcal{H}} \leq 1\right\} \\
& =\sup \left\{\|K h\|_{\mathcal{N}}: h \in \mathcal{H},\|h\|_{\mathcal{H}}=1\right\} \\
& =\sup \left\{\frac{\|K h\|_{\mathcal{N}}}{\|h\|_{\mathcal{H}}}: h \in \mathcal{H},\|h\|_{\mathcal{H}} \neq 0\right\} \\
& =\inf \left\{c:\|K h\|_{\mathcal{N}} \leq c\|h\|_{\mathcal{H}}, \text { for all } h \in \mathcal{H}\right\} .
\end{aligned}
$$

Proof See [84].

Definition Let $\mathcal{H}$ and $\mathcal{N}$ be Hilbert spaces and $K \in \mathcal{B}(\mathcal{H}, \mathcal{N})$. The adjoint of $K$, denoted by $K^{*}$, is the unique operator $A \in \mathcal{B}(\mathcal{N}, \mathcal{H})$ such that $\langle K h, g\rangle_{\mathcal{N}}=\langle h, A g\rangle_{\mathcal{H}}$ for all $h \in \mathcal{H}$ and for all $g \in \mathcal{N}[20]$.

Definition An operator $K \in \mathcal{B}(\mathcal{H})$ is said to be Hermitian if $K=K^{*}$.

Hermitian operators have special properties that will be used in the spectral theorems.

Just as we have an equivalence relation for Hilbert spaces, we would like one for operators in Hilbert spaces. 
Definition Let $\mathcal{H}$ and $\mathcal{N}$ be Hilbert spaces with $A \in \mathcal{B}(\mathcal{H})$ and $B \in \mathcal{B}(\mathcal{N}) . A$ and $B$ are unitarily equivalent if there exists an isomorphism $U: \mathcal{H} \rightarrow \mathcal{N}$ such that

$$
A=U^{-1} B U
$$

Being unitarily equivalent is an equivalence relation for bounded operators on isomorphic Hilbert spaces [20]. If $A$ and $B$ are unitarily equivalent operators, we may consider them the same operator because corresponding elements in $\mathcal{H}$ and $\mathcal{N}$ are mapped to corresponding elements in $\mathcal{H}$ and $\mathcal{N}$ by $A$ and $B$, respectively. In isomorphic Hilbert spaces, we may construct realizations of an operator that are unitarily equivalent. The analysis performed on an operator is valid for any unitarily equivalent operator. In Section 6.1 we show four representations for a Hankel operator, which will be used to simplify the estimation of the singular values of a Hankel operator by causing calculations to be performed on the unitarily equivalent operator for which the calculations are easier.

\subsection{Compact Operators}

Many of the properties of linear operators on finite-dimensional spaces may be generalized to a certain class of linear operators on infinite-dimensional spaces. This class is known as the set of compact operators. 
Definition Let $\mathcal{H}$ and $\mathcal{N}$ be Hilbert spaces and let $H_{1}=\{h \in \mathcal{H}:\|h\| \leq 1\}$. A linear operator $K: \mathcal{H} \rightarrow \mathcal{N}$ is compact if $K\left(H_{1}\right)$ has a compact closure in $\mathcal{N}$. The set of compact operators from $\mathcal{H}$ to $\mathcal{N}$ is denoted $\mathcal{B}_{0}(\mathcal{H}, \mathcal{N})$, and $\mathcal{B}_{0}(\mathcal{H})=\mathcal{B}_{0}(\mathcal{H}, \mathcal{H})$.

Definition The rank of an operator $K$ is the dimension of the range of $K$. $K$ is said to be a finite-rank operator if range $(K)$ is finite dimensional. The set of all finite-rank linear operators from $\mathcal{H}$ to $\mathcal{N}$ is denoted $\mathcal{B}_{00}(\mathcal{H}, \mathcal{N})$, and $\mathcal{B}_{00}(\mathcal{H})=\mathcal{B}_{00}(\mathcal{H}, \mathcal{H})$.

If $K \in \mathcal{B}(\mathcal{H}, \mathcal{N})$, then $K\left(H_{1}\right)$ is bounded; however, in an infinite-dimensional space, this does not imply that the closure of $K\left(H_{1}\right)$ is compact. Thus the definition of compact operators is useful, as it specifies a class of operators that has some properties similar to those of operators on finite-dimensional spaces. Specifically, a useful interpretation is that a compact operator has countable rank.

Theorem 5.3.1 Let $\mathcal{H}$ and $\mathcal{N}$ be Hilbert spaces. Then

1. $\mathcal{B}_{00}(\mathcal{H}, \mathcal{N}) \subset \mathcal{B}_{0}(\mathcal{H}, \mathcal{N}) \subset \mathcal{B}(\mathcal{H}, \mathcal{N})$

2. Let $K \in \mathcal{B}(\mathcal{H}, \mathcal{N})$. Then the following are equivalent:

(a) $K$ is compact.

(b) $K^{*}$ is compact.

(c) There exists $\left\{K_{n}\right\} \subset \mathcal{B}_{00}(\mathcal{H}, \mathcal{N})$ such that $\left\|K-K_{n}\right\| \rightarrow 0$.

(d) There exists $\left\{K_{n}\right\} \subset \mathcal{B}_{0}(\mathcal{H}, \mathcal{N})$ such that $\left\|K-K_{n}\right\| \rightarrow 0$.

Proof See [20] and [10]. 
This theorem indicates that an operator is compact if and only if it can be approximated with any degree of accuracy by a finite-rank operator.

The next result shows that the composition of a compact operator with a bounded operator is compact. Since compact operators are bounded, the composition of two compact operators is itself compact. This theorem is given in [20] for Banach spaces, however, since any Hilbert space is a Banach space, it holds for Hilbert spaces. Theorem 5.3.2 Let $\mathcal{H}, \mathcal{N}$ and $\mathcal{M}$ be Hilbert spaces. Then

1. If $K \in \mathcal{B}_{0}(\mathcal{H}, \mathcal{N})$ and $A \in \mathcal{B}(\mathcal{N}, \mathcal{M})$, then $A K \in \mathcal{B}_{0}(\mathcal{H}, \mathcal{M})$.

2. If $K \in \mathcal{B}_{0}(\mathcal{H}, \mathcal{N})$ and $A \in \mathcal{B}(\mathcal{M}, \mathcal{H})$, then $K A \in \mathcal{B}_{0}(\mathcal{M}, \mathcal{N})$.

Proof See [20].

\subsection{The Spectral Theorem for Compact Hermitian Operators}

The spectral theorem for compact Hermitian operators gives a decomposition of a compact Hermitian operator that is an extension of the diagonalization of a matrix using the eigenvectors of the matrix as a basis. Here we see that the rank of a compact operator is countable. This is a step from uncountably-infinite-rank operators toward finite-rank operators.

Theorem 5.4.1 Let $\mathcal{H}$ be a Hilbert space. $K$ is a compact Hermitian operator on $\mathcal{H}$ if and only if there exists a sequence $\left\{\lambda_{k}\right\}$ of real numbers that tends toward zero and 
an orthonormal basis $\left\{v_{k}\right\}$ of $\mathcal{H}$ such that for any $h \in \mathcal{H}$

$$
K h=\sum_{k=1}^{\infty} \lambda_{k}\left\langle h, v_{k}\right\rangle v_{k}
$$

Proof See [20].

This is called the spectral decomposition of $K$. The elements of the sequence $\left\{\lambda_{k}\right\}$ are called the eigenvalues of the operator, and the basis $\left\{v_{k}\right\}$ are the eigenvectors of the operator. Note that $K v_{k}=\lambda_{k} v_{k}$.

Note that if $K$ is a compact operator, then by Theorem $5.3 .1, K^{*}$ is also a compact operator, and subsequently, by Theorem $5.3 .2, K^{*} K$ is a compact operator. Since $K^{* *}=K$, for all $u, v \in \mathcal{H},\left\langle K^{*} K u, v\right\rangle=\langle K u, K v\rangle=\left\langle u, K^{*} K v\right\rangle$, so $K^{*} K$ is also Hermitian. We now look at the Schmidt expansion of a compact operator.

Theorem 5.4.2 Let $\mathcal{H}$ be a Hilbert space. $K$ acting on $\mathcal{H}$ is compact if and only if there exist orthonormal sequences $\left\{u_{k}\right\}$ and $\left\{w_{k}\right\}$ and scalars $\left\{\sigma_{k}\right\}$ decreasing to 0 such that

$$
K h=\sum_{k=1}^{\infty} \sigma_{k}\left\langle h, u_{k}\right\rangle w_{k}
$$

Proof See [73]. 
This is called the Schmidt decomposition of $K$. The elements of $\left\{\sigma_{k}\right\}$ are the singular values of the operator, and the pairs $\left\{\left(u_{k}, w_{k}\right)\right\}$ are the Schmidt pairs of the operator. $\left\{\sigma_{k}^{2}\right\}$ are the eigenvalues of $K^{*} K$, and $\left\{u_{k}\right\}$ and $\left\{w_{k}\right\}$ are the eigenvectors of $K^{*} K$ and $K K^{*}$ respectively [21]. Further, $K u_{k}=\sigma_{k} w_{k}$ and $K^{*} w_{k}=\sigma_{k} u_{k}$ [21]. An equivalent definition for the singular values is given by [75] in the next theorem. Theorem 5.4.3 Let $\mathcal{H}$ be a Hilbert space and $K \in \mathcal{B}_{0}(\mathcal{H}, \mathcal{N})$. Then the $n^{\text {th }}$ singular value of $K$, denoted $\sigma_{n}(K)$ is given by

$$
\sigma_{n}(K)=\inf \left\{\|K-T\|: T \in \mathcal{B}_{00}(\mathcal{H}, \mathcal{N}), \operatorname{rank}(T)<n\right\}
$$

Proof See [75].

For a compact Hermitian operator $K$, the singular values of $K$ are related to the eigenvalues of $K$, and the Schmidt pairs of $K$ are related to the eigenvectors of $K$.

Theorem 5.4.4 Let $K: \mathcal{H} \rightarrow \mathcal{H}$ be a compact Hermitian operator on a Hilbert space $\mathcal{H}$ with eigenvalues $\left\{\lambda_{k}\right\}$, eigenvectors $\left\{v_{k}\right\}$, singular values $\left\{\sigma_{k}\right\}$, and Schmidt pairs $\left\{\left(u_{k}, w_{k}\right)\right\}$. Then, $\sigma_{k}=\left|\lambda_{k}\right|, u_{k}=v_{k}$, and $w_{k}=\operatorname{sgn}\left(\lambda_{k}\right) v_{k}$ for $k \in \mathbb{N}$.

Proof Because $K$ is a compact Hermitian operator, by Theorem 5.4.1, $K$ has eigenvalues $\left\{\lambda_{k}\right\}$ and eigenvectors $\left\{v_{k}\right\}$. For each $k \in \mathbb{N}$, set $u_{k}=v_{k}, \sigma_{k}=\left|\lambda_{k}\right|$, and 
$w_{k}=\operatorname{sgn}\left(\lambda_{k}\right) v_{k}$. By Theorem 5.4.2, for any $h \in \mathcal{H}$

$$
\begin{aligned}
\sum_{k=1}^{\infty} \sigma_{k}\left\langle h, u_{k}\right\rangle w_{k} & =\sum_{k=1}^{\infty}\left|\lambda_{k}\right|\left\langle h, v_{k}\right\rangle \operatorname{sgn}\left(\lambda_{k}\right) v_{k} \\
& =\sum_{k=1}^{\infty} \operatorname{sgn}\left(\lambda_{k}\right)\left|\lambda_{k}\right|\left\langle h, v_{k}\right\rangle v_{k}=\sum_{k=1}^{\infty} \lambda_{k}\left\langle h, v_{k}\right\rangle v_{k}=K h .
\end{aligned}
$$

Thus we have shown that $\left\{\left|\lambda_{k}\right|\right\} \subset\left\{\sigma_{k}\right\}$ and that $\left\{v_{k}\right\} \subset\left\{u_{k}\right\}$.

Consider $h \in \mathcal{H}$ such that $h \notin\left\{v_{k}\right\}$ and $h \neq 0$. Then, since $\left\{v_{k}\right\}$ is a basis for $\mathcal{H}, h$ can be written as the linear combination of elements in $\left\{v_{k}\right\}$, and $h$ is not orthogonal to at least one $v_{k}$. Hence, $h$ is not orthogonal to at least one $u_{k}$. Since $\left\{u_{k}\right\}$ is also a basis for $\mathcal{H}, h \notin\left\{u_{k}\right\}$. Subsequently, $(h, K h)$ is not a Schmidt pair for $K$. We have shown that the only singular values of $K$ are related to the eigenvalues of $K$, and the only Schmidt pairs of $K$ are related to the eigenvalues of $K$. The result follows.

The Schmidt decomposition fully characterizes a compact operator on a Hilbert space. The Schmidt decomposition is used in the model reduction and $H_{\infty}$ control problems [73].

The ultimate goal of this research is to obtain estimates on the singular values of the Hankel operator for fractional-order systems. A process by which these singular values may be estimated is given in Section 7.1. 


\subsection{Operators on $L_{2}(\mathbb{R})$}

We now move from discussion of operators on a general Hilbert space, and restrict ourselves to operators on a specific Hilbert space, $L_{2}(\mathbb{R})$. Before a discussion of operators on $L_{2}(\mathbb{R})$ can begin, we introduce several spaces related to $L_{2}(\mathbb{R})$. We will be especially interested in the conditions under which Hankel operators on these spaces are unitarily equivalent. Then we will be able to consider the same operator in the space in which the properties and calculation in the space are most convenient.

\subsubsection{Related Spaces}

Definition We define the spaces $L_{2}(\mathbb{R}), L_{2}\left(\mathbb{R}_{+}\right), L_{2}\left(\mathbb{R}_{-}\right)$, and $L_{2}(i \mathbb{R})$ by

$$
\begin{gathered}
L_{2}(\mathbb{R})=\left\{f: \int_{-\infty}^{\infty}|f(t)|^{2} d t<\infty\right\}, \\
L_{2}\left(\mathbb{R}_{+}\right)=\left\{f \in L_{2}(\mathbb{R}): f(t)=0 \text { for } t<0\right\}, \\
L_{2}\left(\mathbb{R}_{-}\right)=\left\{f \in L_{2}(\mathbb{R}): f(t)=0 \text { for } t \geq 0\right\},
\end{gathered}
$$

and

$$
L_{2}(i \mathbb{R})=\left\{F: \int_{-\infty}^{\infty}|F(i \omega)|^{2} d \omega<\infty\right\}
$$

where $F$ is defined on $j \mathbb{R}$, along with the norms

$$
\|f\|_{2}=\left(\int_{-\infty}^{\infty}|f(t)|^{2} d t\right)^{\frac{1}{2}}
$$


for $f \in L_{2}(\mathbb{R}), f \in L_{2}\left(\mathbb{R}_{+}\right)$, or $f \in L_{2}\left(\mathbb{R}_{-}\right)$and

$$
\|F\|_{2}=\left(\frac{1}{2 \pi} \int_{-\infty}^{\infty}|F(i \omega)|^{2} d \omega\right)^{\frac{1}{2}}
$$

for $F \in L_{2}(i \mathbb{R}) . L_{2}(\mathbb{R}), L_{2}\left(\mathbb{R}_{+}\right)$, and $L_{2}\left(\mathbb{R}_{-}\right)$, have been shown to be Hilbert spaces with the usual inner products in Theorem 5.1.1. It can be easily verified that $L_{2}(i \mathbb{R})$ is Hilbert space with inner product

$$
\left\langle F_{1}, F_{2}\right\rangle=\frac{1}{2 \pi} \int_{-\infty}^{\infty} F_{1}(i \omega) \overline{F_{2}(i \omega)} d \omega,
$$

the usual definition of an inner product multiplied by a constant.

For the discussion on operators on $L_{2}(\mathbb{R})$, the spaces $L_{1}(\mathbb{R})$ and $L_{\infty}(i \mathbb{R})$ will prove useful. Thus, we define those spaces here.

Definition Define the space $L_{1}(\mathbb{R})$ by

$$
L_{1}(\mathbb{R})=\left\{f: \int_{-\infty}^{\infty}|f(t)| d t<\infty\right\}
$$

along with the norm

$$
\|f\|_{1}=\int_{-\infty}^{\infty}|f(t)| d t .
$$

for $f \in L_{1}(\mathbb{R})$. Define the space $L_{\infty}(i \mathbb{R})$ by

$$
L_{\infty}(i \mathbb{R})=\{G:|G(i \omega)| \text { is essentially bounded on } i \mathbb{R}\}
$$


along with the norm

$$
\|G\|_{\infty}=\operatorname{ess} \sup \{|G(i \omega)|: \omega \in \mathbb{R}\}
$$

for $G \in L_{\infty}(i \mathbb{R})$.

We wish to show that $L_{2}(\mathbb{R}) \cong L_{2}(i \mathbb{R})$. To do that we require some definitions.

Definition A function $f: \mathbb{R} \rightarrow \mathbb{R}$ is called rapidly decreasing if $f$ is infinitely differentiable and for all $m, n \in \mathbb{N}$

$$
\|f\|_{m, n}=\sup \left\{\left|t^{m} f^{(n)}(t)\right|: t \in \mathbb{R}\right\}<\infty .
$$

The $S$ chwartz space, $\mathcal{S}$ or $\mathcal{S}(\mathbb{R})$, is the set of all rapidly decreasing functions on $\mathbb{R}$.

Recall that a set $A$ is dense in the set $B$ if $A \subset B$ and $B=\bar{A}$. Intuitively, this means that any member of $B$ can be approximated arbitrarily closely by a member of $A$.

Theorem 5.5.1 If $1 \leq p \leq \infty, \mathcal{S} \subset L_{p}(\mathbb{R})$. If $1 \leq p<\infty$, then $\mathcal{S}$ is dense in $L_{p}(\mathbb{R})$.

Proof See [20].

This means that any element of $L_{p}(\mathbb{R})$ can be approximated arbitrarily closely by an element of $\mathcal{S}$. 
The Fourier transform is a well-known tool that will help in showing that $L_{2}(\mathbb{R}) \cong L_{2}(i \mathbb{R})$

Definition Let $f \in L_{1}(\mathbb{R})$. Then the Fourier transform of $f$, denoted $F$, is defined by

$$
F(i \omega)=\int_{-\infty}^{\infty} e^{-i \omega t} f(t) d t
$$

Note that $F(i \omega)$ exists and is finite for all $\omega \in \mathbb{R}$.

Because the Fourier transform is only defined for $f \in L_{1}(\mathbb{R})$, it is not the isomorphism that we are seeking. However, $\mathcal{S} \subset L_{1}(\mathbb{R})$, so the Fourier transform is defined on $\mathcal{S}$, and $\mathcal{S}$ is dense in $L_{2}(\mathbb{R})$. This leads to the isomorphism between $L_{2}(\mathbb{R})$ and $L_{2}(i \mathbb{R})$ that we are seeking.

Theorem 5.5.2 (Plancherel's Theorem) If $f \in \mathcal{S}$, then $\|F\|_{2}=\|f\|_{2}$ and the Fourier transform extends to a surjective isomorphism from $L_{2}(\mathbb{R})$ to $L_{2}(i \mathbb{R})$.

Proof See [20].

This surjective isomorphism is sometimes called the Plancherel transform, and is denoted $\mathcal{F}$. Although, strictly speaking, the Plancherel transform is an extension of the Fourier transform, $\mathcal{F}$ is simply called the Fourier transform. Since $\mathcal{F}$ is an isomorphism, $L_{2}(\mathbb{R}) \cong L_{2}(i \mathbb{R})$. Further, the norms of two elements in $L_{2}(\mathbb{R})$ and $L_{2}(i \mathbb{R})$ related by $\mathcal{F}$ are equal. 
We now establish the relationship between the space $L_{2}(\mathbb{R})$ and the spaces $L_{2}\left(\mathbb{R}_{+}\right)$and $L_{2}\left(\mathbb{R}_{-}\right)$.

Theorem 5.5.3 $L_{2}(\mathbb{R})=L_{2}\left(\mathbb{R}_{+}\right) \oplus L_{2}\left(\mathbb{R}_{-}\right)$

Proof Recall that $L_{2}\left(\mathbb{R}_{+}\right) \subset L_{2}(\mathbb{R})$ and $L_{2}\left(\mathbb{R}_{-}\right) \subset L_{2}(\mathbb{R})$. Note that $L_{2}\left(\mathbb{R}_{+}\right) \cap$ $L_{2}\left(\mathbb{R}_{-}\right)=\{0\}$. Let $f_{+} \in L_{2}\left(\mathbb{R}_{+}\right)$and $f_{-} \in L_{2}\left(\mathbb{R}_{-}\right)$. Then $f=f_{+}+f_{-} \in L_{2}(\mathbb{R})$, so $L_{2}\left(\mathbb{R}_{+}\right) \oplus L_{2}\left(\mathbb{R}_{-}\right) \subset L_{2}(\mathbb{R})$. Let $g \in L_{2}(\mathbb{R})$. Define

$$
g_{+}(t)= \begin{cases}0, & t<0 \\ g(t), & t \geq 0\end{cases}
$$

and

$$
g_{-}(t)=\left\{\begin{array}{ll}
g(t), & t<0 \\
0, & t \geq 0
\end{array} .\right.
$$

It is clear that $g_{-} \in L_{2}\left(\mathbb{R}_{-}\right)$and $g_{+} \in L_{2}\left(\mathbb{R}_{+}\right)$and that $g=g_{-}+g_{+}$. Hence, $L_{2}(\mathbb{R}) \subset L_{2}\left(\mathbb{R}_{+}\right) \oplus L_{2}\left(\mathbb{R}_{-}\right)$. Hence, $L_{2}(\mathbb{R})=L_{2}\left(\mathbb{R}_{+}\right) \oplus L_{2}\left(\mathbb{R}_{-}\right)$.

Lemma 5.5.4 Any function $f$ that has a Fourier transform $F(i \omega)$ has a Laplace transform $F(s)$. Further the two are related by $F(i \omega)=\left.F(s)\right|_{s=i \omega}$.

Proof Let $f$ have a Fourier transform $F(i \omega)=\int_{-\infty}^{\infty} f(t) e^{-i \omega t} d t$. Then the Laplace transform integral $\int_{-\infty}^{\infty} f(t) e^{-s t} d t$ converges for $s=i \omega$ for all $\omega \in \mathbb{R}$, and is exactly the same as the Fourier transform of $f$. 
For a function $f$ with a Fourier transform $F(i \omega), F(s)$ exists and by Theorem 2.2.1 is analytic on $i \mathbb{R}$ since $i \mathbb{R} \subset \mathcal{R}$. Hence $F(s)$ can be obtained via analytic continuation to a unique function [45].

Using the analytic continuation of elements of $L_{2}(i \mathbb{R})$, we now define Hardy spaces $H_{2}\left(\mathbb{C}^{+}\right), H_{2}\left(\mathbb{C}^{-}\right)$, and $H_{\infty}\left(\mathbb{C}^{+}\right)$.

Definition Define $H_{2}\left(\mathbb{C}^{+}\right), H_{2}\left(\mathbb{C}^{-}\right)$, and $H_{\infty}\left(\mathbb{C}^{+}\right)$by

$$
\begin{aligned}
& H_{2}\left(\mathbb{C}^{+}\right)=\left\{F \in L_{2}(i \mathbb{R}): F(s) \text { is analytic in } \mathbb{C}^{+}\right\}, \\
& H_{2}\left(\mathbb{C}^{-}\right)=\left\{F \in L_{2}(i \mathbb{R}): F(s) \text { is analytic in } \mathbb{C}^{-}\right\},
\end{aligned}
$$

and

$$
H_{\infty}\left(\mathbb{C}^{+}\right)=\left\{G \in L_{\infty}(i \mathbb{R}): G(s) \text { is analytic in } \mathbb{C}^{+}\right\}
$$

Note that $H_{2}\left(\mathbb{C}^{+}\right)$and $H_{2}\left(\mathbb{C}^{-}\right)$inherit the inner product and induced norm of $L_{2}(i \mathbb{R})$, and note that $H_{\infty}\left(\mathbb{C}^{+}\right)$inherits the norm of $L_{\infty}(i \mathbb{R}) . H_{2}\left(\mathbb{C}^{+}\right)$and $H_{2}\left(\mathbb{C}^{-}\right)$ are Hilbert spaces [73].

Finally, another space that will prove useful in the discussion of operators on $L_{2}(\mathbb{R})$ is $R H_{\infty}\left(\mathbb{C}^{+}\right)$.

Definition Define the space, $R H_{\infty}\left(\mathbb{C}^{+}\right)$by

$$
R H_{\infty}\left(\mathbb{C}^{+}\right)=H_{\infty}\left(\mathbb{C}^{+}\right) \cap \mathbb{R}(s) .
$$


Since $L_{2}(\mathbb{R}) \cong L_{2}(i \mathbb{R})$ and $L_{2}(\mathbb{R})=L_{2}\left(\mathbb{R}_{+}\right) \oplus L_{2}\left(\mathbb{R}_{-}\right)$, we would like a similar decomposition of $L_{2}(i \mathbb{R})$. The next result shows the correspondence between $L_{2}\left(\mathbb{R}_{+}\right)$and $H_{2}\left(\mathbb{C}^{+}\right)$and between $L_{2}\left(\mathbb{R}_{-}\right)$and $H_{2}\left(\mathbb{C}^{-}\right)$, which does imply that $L_{2}(i \mathbb{R})=H_{2}\left(\mathbb{C}^{+}\right) \oplus H_{2}\left(\mathbb{C}^{-}\right)$.

Theorem 5.5.5 The Laplace transform is an isomorphism from $L_{2}\left(\mathbb{R}_{+}\right)$to $H_{2}\left(\mathbb{C}^{+}\right)$ and from $L_{2}\left(\mathbb{R}_{-}\right)$to $H_{2}\left(\mathbb{C}^{-}\right)$

Proof See [74].

Corollary 5.5.6 $L_{2}\left(\mathbb{R}_{+}\right) \cong H_{2}\left(\mathbb{C}^{+}\right)$and $L_{2}\left(\mathbb{R}_{-}\right) \cong H_{2}\left(\mathbb{C}^{-}\right)$.

Corollary 5.5.7 $L_{2}(i \mathbb{R})=H_{2}\left(\mathbb{C}^{+}\right) \oplus H_{2}\left(\mathbb{C}^{-}\right)$

We define another $L_{2}$-space and related spaces on the unit circle $\mathbb{T}$.

Definition Define the norms $\|f\|_{2}$ and $\|f\|_{\infty}$ by

$$
\|f\|_{2}=\left(\frac{1}{2 \pi} \int_{0}^{2 \pi}\left|f\left(e^{i \theta}\right)\right|^{2} d \theta\right)^{\frac{1}{2}}
$$

and

$$
\|f\|_{\infty}=\operatorname{ess} \sup \left\{\left|f\left(e^{i \theta}\right)\right|: \theta \in[0,2 \pi]\right\} .
$$

Define the spaces $L_{2}(\mathbb{T})$ and $L_{\infty}(\mathbb{T})$ by

$$
L_{2}(\mathbb{T})=\left\{f:\|f\|_{2}<\infty\right\}
$$


and

$$
L_{\infty}(\mathbb{T})=\left\{f:\|f\|_{\infty}<\infty\right\}
$$

The inner product for $L_{2}(\mathbb{T})$ is defined by

$$
\langle f, g\rangle=\frac{1}{2 \pi} \int_{0}^{2 \pi} f\left(e^{i \theta}\right) \overline{g\left(e^{i \theta}\right)} d \theta .
$$

Theorem 5.5.8 The set of functions $\left\{e^{i n \theta}: n \in \mathbb{Z}\right\}$ is a basis for $L_{2}(\mathbb{T})$.

Proof See [73].

Thus, if $f \in L_{2}(\mathbb{T})$, it can be written as a Laurent series in $e^{i \theta}$. Therefore, we can consider $f(z)$ as an analytic function on $\mathbb{T}$, which extends uniquely via analytic continuation to a function on $\mathbb{C}$. We can write $f(z)$ as

$$
f(z)=\sum_{k=-\infty}^{\infty} \hat{\phi}(k) z^{k}
$$

where

$$
\hat{\phi}(k)=\left\langle f, z^{k}\right\rangle \text {. }
$$

We can also define Hardy spaces on the unit disc $\mathbb{D}$. 
Definition Define the norms $\|f\|_{H_{2}}$ and $\|f\|_{H_{\infty}}$ by

$$
\|f\|_{H_{2}}=\left(\frac{1}{2 \pi} \int_{0}^{2 \pi}\left|f\left(e^{i \theta}\right)\right|^{2}\right)^{\frac{1}{2}}
$$

and

$$
\|f\|_{H_{\infty}}=\operatorname{ess} \sup \{|f(z)|: z \in \mathbb{D}\}
$$

Define $H_{2}(\mathbb{D})$ as the space of analytic functions on $\mathbb{D}$ such that $\|f\|_{H_{2}}<\infty$. Similarly, we define $H_{\infty}(\mathbb{D})$ as the space of analytic functions on $\mathbb{D}$ such that $\|f\|_{H_{\infty}}<\infty$.

Definition Let $S \subset \mathbb{Z}$. Define the space $\ell_{2}(S)$ by

$$
\ell_{2}(S)=\left\{\left\{x_{k}\right\}: \sum_{S}\left|x_{k}\right|^{2}<\infty\right\} .
$$

$\ell_{2}(S)$ along with the inner product

$$
\left\langle\left\{a_{k}\right\},\left\{b_{k}\right\}\right\rangle=\sum_{S} a_{k} \overline{b_{k}}
$$

is a Hilbert space.

We note an alternate definition of $H_{2}(\mathbb{D})$.

Theorem 5.5.9 $H_{2}(\mathbb{D})$ can be written as

$$
H_{2}(\mathbb{D})=\left\{f \in L_{2}(\mathbb{T}): \hat{\phi}(k)=0 \text { for all } k<0\right\}
$$


Proof By Theorem 5.5.10 $H_{2}(\mathbb{D}) \subset L_{2}(\mathbb{T})$. Let $f(z) \in L_{2}(\mathbb{T})$. Suppose $\hat{\phi}(k) \neq 0$ for some $k<0$. Then $f(z)$ is not analytic on $\mathbb{D}$ so $f(z) \notin H_{2}(\mathbb{D})$. Thus, $H_{2}(\mathbb{D}) \subset$ $\left\{f \in L_{2}(\mathbb{T}): \hat{\phi}(k)=0\right.$ for all $\left.k<0\right\}$. Suppose that $\hat{\phi}(k)=0$ for all $k<0$. Then $f(z)$ is analytic in $\mathbb{D}$. Thus, $\left\{f \in L_{2}(\mathbb{T}): \hat{\phi}(k)=0\right.$ for all $\left.k<0\right\} \subset H_{2}(\mathbb{D})$ and $H_{2}(\mathbb{D})=\left\{f \in L_{2}(\mathbb{T}): \hat{\phi}(k)=0\right.$ for all $\left.k<0\right\}$.

We now describe the relationships between these spaces and previously defined spaces.

Theorem 5.5.10 The following relationships hold:

1. $L_{2}(\mathbb{R})=L_{2}\left(\mathbb{R}_{-}\right) \oplus L_{2}\left(\mathbb{R}_{+}\right)$

2. $L_{2}(i \mathbb{R})=H_{2}\left(\mathbb{C}^{-}\right) \oplus H_{2}\left(\mathbb{C}^{+}\right)$

3. $L_{2}(\mathbb{T})=H_{2}(\mathbb{D})^{\perp} \oplus H_{2}(\mathbb{D})$

4. $\ell_{2}(\mathbb{Z})=\ell_{2}\left(\mathbb{Z}_{-}\right) \oplus \ell_{2}(\mathbb{N})$

5. $L_{2}(\mathbb{R}) \cong L_{2}(i \mathbb{R}) \cong L_{2}(\mathbb{T}) \cong \ell_{2}(\mathbb{Z})$

6. $L_{2}\left(\mathbb{R}_{+}\right) \cong H_{2}\left(\mathbb{C}^{+}\right) \cong H_{2}(\mathbb{D}) \cong \ell_{2}(\mathbb{N})$

7. $L_{2}\left(\mathbb{R}_{-}\right) \cong H_{2}\left(\mathbb{C}^{-}\right) \cong H_{2}(\mathbb{D})^{\perp} \cong \ell_{2}\left(\mathbb{Z}_{-}\right)$

8. $H_{\infty}(\mathbb{D}) \cong H_{\infty}\left(\mathbb{C}^{+}\right)$

Proof See [73] and [75]. 
We now define two orthogonal projection operators, one from $L_{2}(i \mathbb{R})$ to $H_{2}\left(\mathbb{C}^{+}\right)$and the second from $L_{2}(\mathbb{T})$ to $H_{2}(\mathbb{D})$.

Definition Let $F(s) \in L_{2}(i \mathbb{R})$. There exist unique functions $F_{-}(s) \in H_{2}\left(\mathbb{C}^{-}\right)$and $F_{+}(s) \in H_{2}\left(\mathbb{C}^{+}\right)$such that $F(s)=F_{-}(s)+F_{+}(s)$. Then $\Pi_{+}$the orthogonal projection operator onto $H_{2}\left(\mathbb{C}^{+}\right)$is given by $\Pi_{+} F(s)=F_{+}(s)$, and $\Pi_{-}$the orthogonal projection operator onto $H_{2}\left(\mathbb{C}^{-}\right)$is given by $\Pi_{-} F(s)=F_{-}(s)$.

Definition Let $f(z) \in L_{2}(\mathbb{T})$. There exist unique functions $f_{-}(z) \in H_{2}(\mathbb{D})^{\perp}$ and $f_{+}(z) \in H_{2}(\mathbb{D})$ such that $f(z)=f_{-}(z)+f_{+}(z)$. Then $\Pi_{+}$the orthogonal projection operator onto $H_{2}(\mathbb{D})$ is given by $\Pi_{+} f(z)=f_{+}(z)$, and $\Pi_{-}$the orthogonal projection operator onto $H_{2}(\mathbb{D})^{\perp}$ is given by $\Pi_{-} f(z)=f_{-}(z)$.

It will be clear from the context whether $\Pi_{+}$is the orthogonal projection onto $H_{2}\left(\mathbb{C}^{+}\right)$ or $H_{2}(\mathbb{D})$ and whether $\Pi_{-}$is the orthogonal projection operator onto $H_{2}\left(\mathbb{C}^{-}\right)$or $H_{2}(\mathbb{D})^{\perp}$

Note that $H_{2}(\mathbb{D})$ inherits the inner product of $L_{2}(\mathbb{T})$ and that $H_{2}(\mathbb{D})$ is a Hilbert space [73].

\subsubsection{Convolution Operators on $L_{2}(\mathbb{R})$}

Having built a general abstract framework in which to consider convolution operators, we now discuss such operators on $L_{2}(\mathbb{R})$.

Let $\mathcal{G} \in \mathcal{B}\left(L_{2}(\mathbb{R})\right)$ be the convolution operator with impulse response $\tilde{g}(t)$, that is

$$
(\mathcal{G} u)(t)=\int_{-\infty}^{\infty} \tilde{g}(t-\tau) u(\tau) d \tau
$$


Let $G(s)=\mathcal{L}\{\tilde{g}(t)\}$ be the transfer function for the system whose convolution operator is $\mathcal{G}$.

\section{Theorem 5.5.11}

1. If $\tilde{g} \in L_{1}(\mathbb{R})$, then $G \in L_{\infty}(i \mathbb{R})$ with $\|G\|_{\infty} \leq\|g\|_{1}$.

2. If $\tilde{g} \in L_{1}(\mathbb{R})$ and $\tilde{g}(t)=0$ for $t<0$, then $G \in H_{\infty}\left(\mathbb{C}^{+}\right)$.

\section{Proof}

1. Let $\tilde{g} \in L_{1}(\mathbb{R})$. For any $\omega \in \mathbb{R}$,

$$
\begin{aligned}
|G(i \omega)| & =\left|\int_{-\infty}^{\infty} \tilde{g}(t) e^{-i \omega t} d t\right| \leq \int_{-\infty}^{\infty}\left|\tilde{g}(t) e^{-i \omega t}\right| d t \\
& =\int_{-\infty}^{\infty}|\tilde{g}(t)|\left|e^{-i \omega t}\right| d t=\int_{-\infty}^{\infty}|\tilde{g}(t)| d t \\
& =\|\tilde{g}\|_{1}<\infty .
\end{aligned}
$$

Since $|G(i \omega)| \leq\|\tilde{g}\|_{1}$ for any $\omega \in \mathbb{R},|G(i \omega)|$ has a uniform bound on $i \mathbb{R}$, and it follows that

$$
\|G\|_{\infty}=\operatorname{ess} \sup \{|G(i \omega)|: \omega \in \mathbb{R}\} \leq\|\tilde{g}\|_{1}<\infty .
$$

Thus, $G \in L_{\infty}(i \mathbb{R})$, and $\|G\|_{\infty} \leq\|\tilde{g}\|_{1}$.

2. Let $\tilde{g} \in L_{1}(\mathbb{R})$ and $g(t)=0$ for $t<0$. Since $\tilde{g} \in L_{1}(\mathbb{R})$, by the first part of the theorem, $G \in L_{\infty}(i \mathbb{R})$. Suppose that $s \in \mathbb{C}^{+}$. Hence $\left|e^{-s t}\right| \leq 1$ for $t>0$. 
Then, since $\tilde{g}(t)=0$ for $t<0, G(s)$ is given by

$$
\begin{aligned}
|G(s)| & =\left|\int_{-\infty}^{\infty} \tilde{g}(t) e^{-s t} d t\right|=\left|\int_{0}^{\infty} \tilde{g}(t) e^{-s t} d t\right| \leq \int_{0}^{\infty}\left|\tilde{g}(t) e^{-s t}\right| d t \\
& \leq \int_{0}^{\infty}|\tilde{g}(t)| \cdot 1 d t=\int_{-\infty}^{\infty}|\tilde{g}(t)| d t=\|\tilde{g}\|_{1}<\infty .
\end{aligned}
$$

Thus, the integral converges for $s \in \mathbb{C}^{+}$, so $\mathbb{C}^{+} \subset \mathcal{R}$, and hence by Theorem 2.2.1 $G(s)$ is analytic on $\mathbb{C}^{+}$. Subsequently, $G \in H_{\infty}\left(\mathbb{C}^{+}\right)$.

Theorem 5.5.12 If $G \in L_{\infty}(i \mathbb{R})$, then $\mathcal{G}$ is a bounded operator in $L_{2}(\mathbb{R})$ with $\|\mathcal{G}\|=$ $\|G\|_{\infty}$

Proof Let $u \in L_{2}(\mathbb{R})$ and let $U=\mathcal{F} u \in L_{2}(i \mathbb{R})$. Then

$$
\begin{aligned}
\|\mathcal{G} u\|_{2}^{2} & =\|\mathcal{F}(\mathcal{G} u)\|_{2}^{2}=\|G U\|_{2}^{2}=\frac{1}{2 \pi} \int_{-\infty}^{\infty}|G(i \omega) U(\omega)|^{2} d \omega \\
& =\frac{1}{2 \pi} \int_{-\infty}^{\infty}|G(i \omega)|^{2}|U(\omega)|^{2} d \omega \\
& \leq\|G\|_{\infty}^{2} \frac{1}{2 \pi} \int_{-\infty}^{\infty}|U(\omega)|^{2} d \omega \\
& =\|G\|_{\infty}^{2}\|U\|_{2}^{2}=\|G\|_{\infty}^{2}\|u\|_{2}^{2},
\end{aligned}
$$

Thus, $\mathcal{G} \in \mathcal{B}\left(L_{2}(\mathbb{R})\right)$ with $\|\mathcal{G}\| \leq\|G\|_{\infty}$.

Assume that $G(i \omega) \neq 0$. Let $\epsilon>0$ be given. Define $E$ by

$$
E=\left\{\omega \in \mathbb{R}:|G(i \omega)|>\|G\|_{\infty}-\epsilon\right\}
$$


Clearly, $E$ has positive measure, else

$$
\|G(i \omega)\|_{\infty}=\operatorname{ess} \sup \{|G(i \omega)|: \omega \in \mathbb{R}\} \leq\|G(i \omega)\|_{\infty}-\epsilon
$$

If $E$ has infinite measure, then we wish to replace it with a set that has finite measure. We can choose $I_{\Omega}=[-\Omega, \Omega]$, such that $\hat{E}=E \cap I_{\Omega}$ and $\hat{E}$ has finite, positive measure. We complete the proof replacing $E$ by $\hat{E}$ if $|E|=\infty$. So, without loss of generality, we assume that $|E|<\infty$.

Define $U(i \omega) \in L_{2}(i \mathbb{R})$ by

$$
U(i \omega)=\left\{\begin{array}{cc}
1, & \omega \in E \\
0, & \omega \notin E
\end{array} .\right.
$$

Note that $\|U\|_{2}^{2}=\frac{1}{2 \pi}|E|<\infty$, where $|E|$ is the measure of $E$.

Let $u=\mathcal{F}^{-1}\{U\}$. By Plancherel's theorem, $\|u\|_{2}=\|U\|_{2}$. Then $\|(\mathcal{G} u)(t)\|_{2}^{2}$ is given by

$$
\begin{aligned}
\|\mathcal{G} u\|_{2}^{2} & =\|G U\|_{2}^{2}=\frac{1}{2 \pi} \int_{-\infty}^{\infty}|G(i \omega) U(i \omega)|^{2} d \omega \\
& \geq \frac{1}{2 \pi} \int_{E}|G(i \omega) U(i \omega)|^{2} \geq\left(\|G(i \omega)\|_{\infty}-\epsilon\right)^{2} \frac{1}{2 \pi}|E| \\
& =\left(\|G\|_{\infty}-\epsilon\right)^{2}\|U\|_{2}^{2}=\left(\|G\|_{\infty}-\epsilon\right)^{2}\|u\|_{2}^{2} .
\end{aligned}
$$


Hence,

$$
\frac{\|\mathcal{G} u\|_{2}}{\|u\|_{2}} \geq\|G\|_{\infty}-\epsilon
$$

Since $\epsilon$ is arbitrary, $\|\mathcal{G}\| \geq\|G\|_{\infty}$. Subsequently, $\|\mathcal{G}\|=\|G\|_{\infty}$.

Depending on the region of convergence, $\mathcal{R}$, a first-order transfer function $G(s)$ with its pole at $s_{+} \in \mathbb{C}_{+}$can be interpreted in two ways. Note that, by Theorem 2.2.1, $G(s)$ cannot contain any poles in $\mathcal{R}$ and that $G(s)$ is analytic in $\mathcal{R}$.

The first interpretation is that $\mathcal{R}$ is to the right of the vertical line containing $s_{+}$. Since $s_{+} \in \mathbb{C}^{+}, i \mathbb{R} \not \subset \mathcal{R}$. Hence, $\mathcal{G} \notin \mathcal{B}\left(L_{2}(\mathbb{R})\right)$. The second interpretation is that $\mathcal{R}$ is to the left of the vertical line containing $s_{+}$. Since $s_{+} \in \mathbb{C}^{+}, i \mathbb{R} \subset \mathcal{R}$. Hence, $\mathcal{G} \in \mathcal{B}\left(L_{2}(\mathbb{R})\right)$. Further, since $G(s)$ is analytic in $\mathbb{C}^{-}, G(s) \in H_{2}\left(\mathbb{C}^{-}\right)$, which means that $g(t) \in L_{2}\left(\mathbb{R}_{-}\right)$. Thus, $\mathcal{G}$ is anticausal. Because we wish to consider only operators in $\mathcal{B}\left(L_{2}(\mathbb{R})\right)$, we use this second interpretation.

\subsection{Integral Operators on $L_{2}(\mathbb{R})$}

We now consider a slightly broader class of operators on a function space, which we will take to be $L_{2}(\mathbb{R})$. In Chapter 6 , it will be shown that Hankel operators 
are integral operators, so these results will apply to both convolution and Hankel operators.

Definition Let $E$ be measurable. An integral operator on a function space is an operator of the form $(K u)(t)=\int_{E} \tilde{g}(t, \tau) u(\tau) d \tau . \tilde{g}(t, \tau)$ is called the kernel of the integral operator.

Definition An integral equation is an equation in which an unknown function appears inside an integral sign. An alternate definition is that an integral equation is an equation involving an integral operator acting on an unknown function.

\subsubsection{General Discussion}

As noted $L_{2}(\mathbb{R})$ is a Hilbert space. Consider the integral operator $K$ on $L_{2}(\mathbb{R})$ given by

$$
(K u)(t)=\int_{0}^{\infty} g(t, \tau) u(\tau) d \tau
$$

Information about the kernel of the operator yields information about the properties of the operator. The following theorem from [76] gives a sufficient condition on the kernel for an integral operator to be bounded.

Theorem 5.6.1 Consider $g(t, \tau): E \times E \rightarrow \mathbb{F}$, and suppose that there exist constants $c_{1}$ and $c_{2}$ such that

$$
\int_{E}|g(t, \tau)| d \tau<c_{1}
$$

and

$$
\int_{E}|g(t, \tau)| d t<c_{2}
$$


Then the integral operator $K: L_{2}(E) \rightarrow L_{2}(E)$ with kernel $g(t, \tau)$ is a bounded linear operator with $\|K\| \leq \sqrt{c_{1} c_{2}}$.

Proof See [76].

We are interested in integral operators on $L_{2}(E)$. We particularly want to work with bounded linear operators on $L_{2}(E)$, which are operators from $L_{2}(E)$ to $L_{2}(E)$. The relationship between the kernels of an integral operator and its adjoint operator is given in the next theorem.

Theorem 5.6.2 Consider the operator $K: L_{2}(E) \rightarrow L_{2}(E)$ with kernel $g(t, \tau)$. Then the adjoint of $K, K^{*}$, is the integral operator with kernel $\overline{g(\tau, t)}$.

Proof See [20].

By this theorem, if $g(t, \tau)=\overline{g(\tau, t)}$, then the integral operator is Hermitian. A sufficient, but not necessary, condition on the kernel for the integral operator to be compact is given by [39].

Theorem 5.6.3 Consider the operator $K: L_{2}(E) \rightarrow L_{2}(E)$ with kernel $g(t, \tau)$. If $g(t, \tau) \in L_{2}(E \times E)$, then $K$ is a compact operator with $\|K\| \leq\|g\|_{2}$. Such a kernel is called an $L_{2}-$ kernel.

Proof See [39]. 
Where possible, these results will be used to demonstrate that a particular integral operator is bounded, Hermitian, or compact.

\subsubsection{Degenerate Kernels}

Definition The kernel of an integral operator is called degenerate or separable if there exist two finite sets of functions, $\left\{\alpha_{k}(t)\right\}_{k=1}^{N}$ and $\left\{\beta_{k}(\tau)\right\}_{k=1}^{N}$ such that

$$
g(t, \tau)=\sum_{k=1}^{N} \alpha_{k}(t) \beta_{k}(\tau) .
$$

Definition The kernel of an integral operator is called semi-degenerate or semiseparable if there exist four finite sets of function, $\left\{\alpha_{k}(t)\right\}_{k=1}^{N},\left\{\beta_{k}(t)\right\}_{k=1}^{N},\left\{\eta_{k}(t)\right\}_{k=1}^{M}$, and $\left\{\zeta_{k}(t)\right\}_{k=1}^{M}$ such that

$$
g(t, \tau)=\left\{\begin{array}{ll}
\sum_{k=1}^{N} \alpha_{k}(t) \beta_{k}(\tau), & t>\tau \\
\sum_{k=1}^{M} \eta_{k}(t) \zeta_{k}(\tau), & t<\tau
\end{array} .\right.
$$

For integral operators on $L_{2}(E)$ for any measurable set $E$, being finite-rank is equivalent to having a degenerate kernel. We focus on the case where $E$ as a subset of $\mathbb{R}$.

Theorem 5.6.4 Let $E \in\left\{\mathbb{R}, \mathbb{R}_{+}, \mathbb{R}_{-}\right\}$and let $K: L_{2}(E) \rightarrow L_{2}(E)$ be an integral operator with kernel $g(t, \tau) . K$ is a finite-rank operator on $L_{2}(E)$ if and only if $g(t, \tau)$ is degenerate.

Proof See [5] and [78]. 
This theorem indicates that integral operators with semi-degenerate kernels are infinite-rank operators. Calculating $K u$ is simplified somewhat if $K$ has a semidegenerate kernel.

\subsubsection{Application to Ordinary Integer-Order Differential Equations}

Recall that a system described by an integer-order differential equation can be represented by a convolution operator,

$$
x(t)=(\mathcal{G} u)(t)=\int_{-\infty}^{\infty} \tilde{g}(t-\tau) u(\tau) d \tau .
$$

Clearly, this is an integral operator. If $\tilde{g}(t) \in L_{1}(\mathbb{R})$, then by Theorem $5.6 .1, \mathcal{G}$ is a bounded operator on $L_{2}(\mathbb{R})$. For real-world applications, signals cannot have infinite energy, so it is natural to consider systems as operators from $L_{2}(\mathbb{R})$ to $L_{2}(\mathbb{R})$. Thus, we consider systems as integral operators on $L_{2}(\mathbb{R})$. Note that by Equation 2.12, the kernel of a convolution operator $\mathcal{G}$ arising from an integer-order system can be written as

$$
\tilde{g}(t)=\sum_{k=1}^{k_{n}} \sum_{i=1}^{m_{k}} r_{k_{i}} \frac{t^{i-1} e^{p_{k} t}}{(i-1) !} \mathcal{U}_{k}(t)
$$

If the system is causal and has only simple poles, Equation 5.5 simplifies to

$$
\tilde{g}(t)=\sum_{k=1}^{n} r_{k_{i}} e^{p_{k}(t-\tau)} \mathcal{U}_{s}(t-\tau)
$$


Let $\alpha_{k}(t)=r_{k} e^{p_{k} t}$ and $\beta_{k}(\tau)=e^{p_{k} \tau}$. Then the convolution operator for the system can be written as

$$
(\mathcal{G} f)(t)=\int_{-\infty}^{\infty} \sum_{k=1}^{n} \alpha_{k}(t) \beta_{k}(\tau) \mathcal{U}_{s}(t-\tau) f(\tau) d \tau
$$

Note that the kernel is not degenerate, but only semi-degenerate. Thus, the operator is of infinite-rank. However, for $t>0$, the integral may be divided into the initialcondition response and the forced response as

$$
\begin{aligned}
(\mathcal{G} f)(t)= & \int_{-\infty}^{0} \sum_{k=1}^{n} \alpha_{k}(t) \beta_{k}(\tau) \mathcal{U}_{s}(t-\tau) f(\tau) d \tau \\
& +\int_{0}^{\infty} \sum_{k=1}^{n} \alpha_{k}(t) \beta_{k}(\tau) \mathcal{U}_{s}(t-\tau) f(\tau) d \tau \\
= & \int_{-\infty}^{0} \sum_{k=1}^{n} \alpha_{k}(t) \beta_{k}(\tau) f(\tau) d \tau+\int_{0}^{\infty} \sum_{k=1}^{n} \alpha_{k}(t) \beta_{k}(\tau) \mathcal{U}_{s}(t-\tau) f(\tau) d \tau
\end{aligned}
$$

since $\mathcal{U}_{s}(t-\tau)=1$ for $t>0$ and $\tau<0$. The operation associated with the forced response still only has a semi-degenerate kernel and is thus infinite-rank. However, the initial condition response has a degenerate kernel and is thus finite-rank. A similar argument can be made for integer-order systems with repeated poles. It will be shown in Theorem 6.3.1 that for a fractional-order system the initial condition operator is of infinite rank. Because of the presence of $\mathcal{U}_{s}(t-\tau)$ in the kernel of the forced response, the associated operator is not Hermitian by Theorem 5.6.2. For many systems the initial condition operator turns out to be Hermitian. We will see 
the conditions on the impulse response that give Hermitian initial condition operators in Section 6.1.

5.7 Estimation of Eigenvalues for Operators on a Hilbert Space

The following methods may be used for estimating eigenvalues and eigenvectors of compact operators on any Hilbert space.

\subsubsection{The Rayleigh-Ritz Method}

This method of obtaining eigenvalue estimates was originated by Rayleigh [80] and Ritz [81].

Definition Let $\mathcal{H}$ be a Hilbert space, and let $K \in \mathcal{B}(\mathcal{H})$. Define the functionals, $J_{K}(f)$ and $R_{K}(f)$ by

$$
J_{K}(f)=\langle K f, f\rangle
$$

and

$$
R_{K}(f)=\frac{\langle K f, f\rangle}{\|f\|^{2}}
$$

for all $f \in \mathcal{H} . R_{K}(f)$ is called the Rayleigh quotient.

The heart of the Rayleigh-Ritz method is in the following lemma.

Lemma 5.7.1 Let $\mathcal{H}$ be a Hilbert space, and let $K \in \mathcal{B}_{0}(\mathcal{H})$ be a compact Hermitian operator, and let $f \in \mathcal{H}$ such that $\|f\|=1$. Then $f$ is a stationary point of $\langle K f, f\rangle$ if 
and only if $f$ is an eigenfunction of $K$. The stationary values are the corresponding eigenvalues of $K$.

Proof See [78].

The Rayleigh-Ritz method involves selecting a finite basis $\left\{\phi_{k}\right\}_{k=1}^{N}$, the span of which is the subspace $E_{N} \subset \mathcal{H}$. Elements of $\mathcal{H}$ are approximated by elements in $E_{N}$. Then the stationary points and values of $J_{K}(p)$ for $p \in E_{N}$ such that $\|p\|=1$ are found. These approximate the stationary points and values of $J_{K}(f)$ for $f \in \mathcal{H}$, which by Lemma 5.7.1 are the eigenvectors and eigenvalues of the operator $K$. When the eigenvalue that is being approximated is nonzero, the approximation $\hat{\lambda}_{k}$ is no larger in magnitude than the actual eigenvalue $\lambda_{k}$; that is, $\left|\hat{\lambda}_{k}\right| \leq\left|\lambda_{k}\right|$ if $\lambda_{k} \neq 0$ [78]. We call these estimates underestimates.

Computationally, the essence of the Rayleigh-Ritz method is that an eigenvector problem in $\mathcal{H}$ is replaced by a generalized eigenvector problem in $E_{N}$. This is possible because the stationary points of $J_{K}(p)$ for $p \in E_{N}$ restricted by $\|p\|=1$ for $1 \leq m \leq N$ are $p_{m}=\sum_{k=1}^{N} \alpha_{k m} \phi_{k}$, where the $\alpha_{k m}$ are the solutions of the system of equations

$$
\sum_{k=1}^{N}\left(J_{\ell k}-\mu_{m} I_{\ell k}\right) \alpha_{k m}=0
$$

for $1 \leq \ell \leq N$, with $J_{\ell k}=\left\langle K \phi_{k}, \phi_{\ell}\right\rangle$ and $I_{\ell k}=\left\langle\phi_{k}, \phi_{\ell}\right\rangle$ [78]. The $\mu_{m}$ are the stationary values corresponding to the $p_{m}$. Computationally, the stationarity of the $p_{m}$ and $\mu_{m}$ 
is unimportant. However, this stationarity in instrumental in proving Theorem 5.7.3. The system of equations in Equation 5.6 can be written as

$$
P \alpha_{m}=\mu_{m} Q \alpha_{m},
$$

where

$$
\begin{gathered}
P=\left(\begin{array}{cccc}
\left\langle K \phi_{1}, \phi_{1}\right\rangle & \left\langle K \phi_{1}, \phi_{2}\right\rangle & \cdots & \left\langle K \phi_{1}, \phi_{N}\right\rangle \\
\left\langle K \phi_{2}, \phi_{1}\right\rangle & \left\langle K \phi_{2}, \phi_{2}\right\rangle & \cdots & \left\langle K \phi_{2}, \phi_{N}\right\rangle \\
\vdots & \vdots & \ddots & \vdots \\
\left\langle K \phi_{N}, \phi_{1}\right\rangle & \left\langle K \phi_{N}, \phi_{2}\right\rangle & \cdots & \left\langle K \phi_{N}, \phi_{N}\right\rangle
\end{array}\right) \\
\alpha_{m}=\left(\begin{array}{c}
\alpha_{1 m} \\
\alpha_{2 m} \\
\vdots \\
\alpha_{N m}
\end{array}\right)
\end{gathered}
$$

and

$$
Q=\left(\begin{array}{cccc}
\left\langle\phi_{1}, \phi_{1}\right\rangle & \left\langle\phi_{1}, \phi_{2}\right\rangle & \cdots & \left\langle\phi_{1}, \phi_{N}\right\rangle \\
\left\langle\phi_{2}, \phi_{1}\right\rangle & \left\langle\phi_{2}, \phi_{2}\right\rangle & \cdots & \left\langle\phi_{2}, \phi_{N}\right\rangle \\
\vdots & \vdots & \ddots & \vdots \\
\left\langle\phi_{N}, \phi_{1}\right\rangle & \left\langle\phi_{N}, \phi_{2}\right\rangle & \cdots & \left\langle\phi_{N}, \phi_{N}\right\rangle
\end{array}\right)
$$

To summarize, the $\alpha_{k m}$ are the entries of the generalized eigenvectors of $P$ with respect to $Q$, where the $(\ell, k)$ entry of $P$ is $J_{\ell k}$ and the $(\ell, k)$ entry of $Q$ is $I_{\ell k}$. The $\mu_{m}$ are the corresponding generalized eigenvalues of $P$ with respect to $Q$. 


\subsubsection{The Galerkin Method}

The Galerkin method is a slightly different approach to the eigenvalue estimation proposed by Galerkin [26]. As in the Rayleigh-Ritz method, a finite-dimensional subspace $E_{N} \subset \mathcal{H}$ is used to determine approximations to the eigenvalues of the Hermitian operator $K \in \mathcal{B}_{0}(\mathcal{H})$. Rather than approximating elements in $\mathcal{H}$ with elements in $E_{N}=\operatorname{span}\left\{\phi_{k}\right\}_{k=1}^{N}$, the Galerkin method approximates the operator $K$ with $\hat{K}=P_{N} K P_{N}$, where $P_{N}$ is the orthogonal projection operator from $\mathcal{H}$ to $E_{N}$. For $p \in \mathcal{H}$ the eigenvalues and eigenvectors of $\hat{K}$ satisfy

$$
\mu p=\hat{K} p .
$$

Since it is clear that if $p_{m}$ is an eigenvector of $\hat{K}$ then $p_{m} \in E_{N}$, we can make the substitution $p_{m}=\sum_{k=1}^{N} \alpha_{k m} \phi_{k}$, which gives the system of equations

$$
\sum_{k=1}^{N}\left(J_{\ell k}-\mu_{m} I_{\ell k}\right) \alpha_{k m}=0
$$

for $1 \leq \ell \leq N$ as in the Rayleigh-Ritz method. The $\alpha_{k m}$ are the entries of the generalized eigenvectors of $P$ with respect to $Q$, where the $(\ell, k)$ entry of $P$ is $J_{\ell k}$ and the $(\ell, k)$ entry of $Q$ is $I_{\ell k}$. The $\mu_{m}$ are the corresponding generalized eigenvalues of $P$ with respect to $Q$. The eigenvalues and eigenvectors of $\hat{K}$, the projected operator, are determined from $\alpha_{k m}$ and $\mu_{m}$. The eigenvalues and eigenvectors of $\hat{K}$ are approximates to those of $K$. Although the Rayleigh-Ritz method involves approximating 
the eigenvectors of $K$ and the Galerkin method involves approximating $K$ itself, the computations involved in both methods are the same.

\subsubsection{Overestimates on Eigenvalues}

The Rayleigh-Ritz method produces underestimates of positive eigenvalues. We desire to have bounded estimates on the Hankel singular values, that is both an underestimate and an overestimate. Recall that $J_{K}(p)$ is defined by

$$
J_{K}(p)=\langle K p, p\rangle
$$

The following lemma will be used to facilitate an easier calculation for the overestimates.

Lemma 5.7.2 Let $K \in \mathcal{B}_{0}(\mathcal{H})$ be a Hermitian operator. Then

$$
J_{K^{2}}(p)-J_{K}^{2}(p)=\left\|K p-J_{K}(p) p\right\|^{2}
$$


Proof Note that $J_{K}(p) \in \mathbb{R}$. Then,

$$
\begin{aligned}
J_{K^{2}}(p)-J_{K}^{2}(p) & =\left\langle K^{2} p, p\right\rangle-J_{K}(p) \overline{J_{K}(p)} \\
& =\langle K p, K p\rangle-J_{K}(p) \overline{J_{K}(p)}-J_{K}(p) \overline{J_{K}(p)}+J_{K}^{2}(p) \\
& =\langle K p, K p\rangle-\langle K p, p\rangle \overline{J_{K}(p)}-J_{K}(p)\langle p, K p\rangle+J_{K}^{2}(p)\langle p, p\rangle \\
& =\langle K p, K p\rangle-\left\langle K p, J_{K}(p) p\right\rangle-\left\langle J_{K}(p) p, K p\right\rangle+\left\langle J_{K}(p) p, J_{K}(p) p\right\rangle \\
& =\left\langle K p-J_{K}(p) p, K p-J_{K}(p) p\right\rangle . \\
& =\left\|K p-J_{K}(p) p\right\|^{2}
\end{aligned}
$$

Theorem 5.7.3 Let $K \in \mathcal{B}_{0}(\mathcal{H})$ be a Hermitian operator. Then for any $p \in \mathcal{H}$ such that $\|p\|=1, K$ has at least one eigenvalue in the interval I given by

$$
I=\left[J_{K}(p)-\left\|K p-J_{K}(p) p\right\|, J_{K}(p)+\left\|K p-J_{K}(p) p\right\|\right] .
$$

Proof See [78].

The proof of Theorem 5.7.3 makes use of the fact that the eigenvectors of $K$ are the stationary points of $J_{K}(p)$. 
Theorem 5.7.3 along with the Rayleigh-Ritz method allows the calculation of bounded eigenvalue estimates. For a compact Hermitian operator $K$, we apply the Rayleigh-Ritz method to determine an underestimate $\mu_{k}$ to the eigenvalue $\lambda_{k}$ of $K$ and an $N$-dimensional approximate $p_{k}$ to a corresponding eigenvector $\phi_{k}$ of $K$, noting that $\mu_{k}=J_{K}\left(p_{k}\right)$. Then, using $p_{k}$, we apply Theorem 5.7 .3 to obtain an overestimate, $\nu_{k}$ of the eigenvalue $\lambda_{k}$ of $K$, given by $\nu_{k}=J_{K}\left(p_{k}\right)+\left\|K p_{k}-J_{K}\left(p_{k}\right) p_{k}\right\|$. If the bounds are not sufficiently close, we increase the value of $N$ and repeat the process.

\subsection{Examples}

We now look at the second-order system, the resistor-terminated, and inductorterminated semi-infinite lines as convolution operators on $L_{2}(\mathbb{R})$.

\subsubsection{Second-Order System}

Recall the second order system introduced in Section 2.4.1, whose transfer function is

$$
G(s)=\frac{-2 s}{s^{2}+s+\frac{1}{4}},
$$

and whose convolution kernel is

$$
\begin{aligned}
\tilde{g}(t-\tau) & =e^{-\frac{t-\tau}{2}}(t-\tau-2) \mathcal{U}_{s}(t-\tau) \\
& =\left(e^{-\frac{t}{2}}(t-2) e^{\frac{\tau}{2}}+e^{-\frac{t}{2}}\left(-\tau e^{\frac{\tau}{2}}\right)\right) \mathcal{U}_{s}(t-\tau)
\end{aligned}
$$


Note that

$$
\int_{-\infty}^{\infty}|g(t-\tau)| d \tau=\frac{8}{e}=c_{1}
$$

and

$$
\int_{-\infty}^{\infty}|g(t-\tau)| d t=\frac{8}{e}=c_{2}
$$

Hence, by Theorem 5.6.1, the convolution operator $\mathcal{G}$ whose kernel is $\tilde{g}(t-\tau)$ is a bounded operator with $\|\mathcal{G}\| \leq \frac{8}{e}$. Note that $G \in L_{\infty}(i \mathbb{R})$ with a maximum value $\|G\|_{\infty}=2$. Hence, by Theorem 5.5.12, $\|\mathcal{G}\|=2<\frac{8}{e}$.

Because $\tilde{g}(t-\tau) \notin L_{2}\left(\mathbb{R}^{2}\right)$, we cannot use Theorem 5.6.3 to determine the compactness of $\mathcal{G}$. Also note that although $\tilde{g}(t-\tau)$ is semi-degenerate, it is not degenerate, so $\mathcal{G}$ is of infinite rank by Theorem 5.6.4. By Theorem 6.5.12, since $\tilde{g}(t-\tau) \neq \tilde{g}(\tau-t), \mathcal{G}$ is not Hermitian. Because the convolution operator is an integral operator with an upper limit $t$, it has no non-zero singular values [82].

Consider the initial-condition response and the forced response given in Equations 2.20 and 2.21,

$$
\begin{aligned}
x_{i c}(t) & =\int_{-\infty}^{0} e^{-\frac{t-\tau}{2}}(t-\tau-2) f(\tau) d \tau \\
& =\int_{-\infty}^{0}\left(e^{-\frac{t}{2}}(t-2) e^{\frac{\tau}{2}}+e^{-\frac{t}{2}}\left(-\tau e^{\frac{\tau}{2}}\right)\right) f(\tau) d \tau
\end{aligned}
$$


and

$$
\begin{aligned}
x_{f}(t) & =\int_{0}^{t} e^{-\frac{t-\tau}{2}}(t-\tau-2) f(\tau) d \tau \\
& =\int_{0}^{t}\left(e^{-\frac{t}{2}}(t-2) e^{\frac{\tau}{2}}+e^{-\frac{t}{2}}\left(-\tau e^{\frac{\tau}{2}}\right)\right) f(\tau) d \tau \\
& =\int_{0}^{\infty}\left(e^{-\frac{t}{2}}(t-2) e^{\frac{\tau}{2}}+e^{-\frac{t}{2}}\left(-\tau e^{\frac{\tau}{2}}\right)\right) \mathcal{U}_{s}(t-\tau) f(\tau) d \tau
\end{aligned}
$$

As with $\mathcal{G}$ the operator associated with the forced response has a semi-degenerate, but not degenerate, kernel. Hence, the operator associated with the forced response is an infinite-rank operator. However, in this case, the operator associated with the initial-condition response has a degenerate kernel because the kernel can be written as $\alpha_{1}(t) \beta_{1}(\tau)+\alpha_{2}(t) \beta_{2}(\tau)$, where $\alpha_{1}(t)=e^{-\frac{t}{2}}(t-2), \beta_{1}(\tau)=e^{\frac{\tau}{2}}, \alpha_{2}(t)=e^{-\frac{t}{2}}$, and $\beta_{2}(\tau)=e^{\frac{\tau}{2}} \tau$. Thus, the associated operator is finite-rank, and is also compact. This is a specific case of the general discussion in Section 5.6.3.

To find the singular values $\left\{\sigma_{k}\right\}$ and Schmidt pairs $\left\{\left(u_{k}, w_{k}\right)\right\}$ of the initial condition operator, $\left\{w_{k}(t)\right\}$ can be selected as the orthonormalization of $\left\{\alpha_{k}(t)\right\}$, and $\left\{u_{k}(\tau)\right\}$ can be selected as the orthonormalization of $\left\{\beta_{k}(\tau)\right\}$. With this in mind, we set $w_{1}(t)=e^{-\frac{t}{2}}(t-1)$ and $w_{2}(t)=-e^{-\frac{t}{2}}$ for $t \geq 0$, and set $u_{1}(\tau)=e^{\frac{\tau}{2}}$ and $u_{2}(\tau)=e^{\frac{\tau}{2}}(\tau+1)$ for $\tau<0$. Note that $w_{1}$ and $w_{2}$ are orthonormal on $L_{2}\left(\mathbb{R}_{+}\right)$and that $u_{1}$ and $u_{2}$ are orthonormal on $L_{2}\left(\mathbb{R}_{-}\right)$. We can rewrite Equation 5.10 as

$$
\begin{aligned}
x_{i c}(t) & =\int_{-\infty}^{0}\left(e^{-\frac{t}{2}}(t-1) e^{\frac{\tau}{2}}-e^{-\frac{t}{2}} e^{\frac{\tau}{2}}(\tau+1)\right) f(\tau) d \tau \\
& =e^{-\frac{t}{2}}(t-1) \int_{-\infty}^{0} e^{\frac{\tau}{2}} f(\tau) d \tau-e^{-\frac{t}{2}} \int_{-\infty}^{0} e^{\frac{\tau}{2}}(\tau+1) f(\tau) d \tau .
\end{aligned}
$$


Then for $k \in\{1,2\},\left(u_{k}, w_{k}\right)$ are the Schmidt pairs of the initial-condition operator and $\sigma_{1}=1=\sigma_{2}$ are the only non-zero singular values of the initial-condition operator. Hence, this operator has a rank of 2. We will see in Chapter 6 that the operator associated with the initial-condition response is a Hermitian operator.

\subsubsection{Resistor-Terminated Semi-Infinite Line}

Recall that the resistor-terminated semi-infinite line has transfer function

$$
G_{R}(s)=\frac{1}{s^{\frac{1}{2}}+1}
$$

and convolution kernel

$$
\tilde{g}_{R}(t-\tau)=\sum_{k=1}^{2}(-1)^{k-1} E_{t-\tau}\left(\frac{k-2}{2}, 1\right) \mathcal{U}_{s}(t-\tau)
$$

$G_{R} \in L_{\infty}(i \mathbb{R})$ with $\|G\|_{\infty}=1$. Hence, $\left\|\mathcal{G}_{R}\right\|=1$ by Theorem 5.5.12. Because $E_{t-\tau}\left(-\frac{1}{2}, 1\right)$ is neither degenerate nor semi-degenerate, $\tilde{g}_{R}(t-\tau)$ is neither degenerate nor semi-degenerate. Thus, $\mathcal{G}_{R}$ and the operators associated with the initial-condition and forced responses are of infinite rank. Because $\tilde{g}_{R}(t-\tau) \neq \tilde{g}_{R}(\tau-t)$, by Theorem 6.5.12 is not Hermitian. We can do no simple analysis on the convolution operator or the operators associated with the initial-condition and forced responses to determine a Schmidt decomposition. 


\subsubsection{Inductor-Terminated Semi-Infinite Line}

The transfer function of the inductor-terminated semi-infinite line can be written as

$$
G_{L}(s)=\frac{1}{s^{\frac{3}{2}}+1}=\frac{\frac{1}{3}}{s^{\frac{1}{2}}+1}-\frac{\frac{s^{\frac{1}{2}}-2}{3}}{s-s^{\frac{1}{2}}+1} .
$$

$G_{L} \in L_{\infty}(i \mathbb{R})$ with $\|G\|_{\infty}=\sqrt{2}$, so by Theorem 5.5.12, $\left\|\mathcal{G}_{L}\right\|=\sqrt{2}$. Note that in

Equation 5.12, $\frac{1}{s^{\frac{1}{2}}+1}$ appears. Since this term leads to a non-degenerate convolution kernel, the entire convolution kernel is non-degenerate. Again, the kernel is not even semi-degenerate. Since $\tilde{g}_{L}(t-\tau) \neq \tilde{g}(\tau-t)$, by Theorem 6.5.12 $\mathcal{G}_{L}$ is not Hermitian. Once again, there is very little that can be said about this operator.

\subsection{Conclusions}

For the second-order system, the forced response was difficult to analyze, and no decomposition for the associated operator was found. However, it was seen that the operator associated with the initial condition response is finite-rank, and a Schmidt decomposition was obtained. For the resistor-terminated and inductor-terminated semi-infinite lines, the convolution operators and operators associated with the forced and initial-condition responses were found to be infinite-rank. It will be seen in Chapter 6 that the operators associated with the initial-condition responses, while of infinite rank, are compact. Because compact operators are more easily analyzed, 
we study the operator which takes inputs for $t<0$ to outputs for $t>0$, called the Hankel operator. 


\section{CHAPTER VI}

\section{HANKEL OPERATORS}

We have provided a general abstract framework in which convolution operators on $L_{2}(\mathbb{R})$ can be considered. We now introduce the Hankel operator, which maps inputs for $t<0$ to outputs for $t>0$. On $L_{2}\left(\mathbb{R}_{+}\right)$the Hankel operator for a system is closely related to the convolution operator on $L_{2}(\mathbb{R})$ for that system. In this chapter equivalent Hankel operators are defined on $L_{2}\left(\mathbb{R}_{+}\right), H_{2}\left(\mathbb{C}^{+}\right), H_{2}(\mathbb{D})$, and $\ell_{2}(\mathbb{N})$. Next, some general results about Hankel operators are presented. We demonstrate the conditions under which the class of fractional-order operators with transfer function $G(s)=\frac{r}{s^{q}-a}+\frac{\bar{r}}{s^{\bar{q}}-\bar{a}}$ have compact Hankel operators. A final result shows that when $q$ is not purely imaginary, the Hankel operator associated with $G(s)$ is either compact or unbounded.

\subsection{Representations of Hankel Operators}

On each of the Hilbert spaces $L_{2}\left(\mathbb{R}_{+}\right), H_{2}\left(\mathbb{C}^{+}\right), H_{2}(\mathbb{D})$, and $\ell_{2}(\mathbb{N})$ we define an operator that we call a Hankel operator. 
We would like an operator that maps inputs for time $t<0$ to outputs for time $t>0$. For a function $f \in L_{2}(\mathbb{R})$, we define $f_{-}$by

$$
f_{-}(t)=\left\{\begin{array}{ll}
f(t), & t<0 \\
0, & t>0
\end{array},\right.
$$

where $f_{-}(t)$ represents the input for time $t<0$. Note that $f_{-}(t) \in L_{2}\left(\mathbb{R}_{-}\right)$and $f_{-}(-t) \in L_{2}\left(\mathbb{R}_{+}\right)$. Consider $\mathcal{G} f_{-}$, which is given by

$$
\begin{aligned}
\left(\mathcal{G} f_{-}\right)(t) & =\int_{-\infty}^{\infty} \tilde{g}(t-\tau) f_{-}(\tau) d \tau=\int_{-\infty}^{0} \tilde{g}(t-\tau) f_{-}(\tau) d \tau \\
& =\int_{0}^{\infty} \tilde{g}(t+\tau) f_{-}(-\tau) d \tau
\end{aligned}
$$

under the mapping $\tau \mapsto-\tau$. Comparing Equation 6.1 with Equation 2.15, it is clear that this is a form of the initial-condition response of $\mathcal{G}$.

We define the Hankel operator $\Gamma_{\mathcal{G}}: L_{2}\left(\mathbb{R}_{+}\right) \rightarrow L_{2}\left(\mathbb{R}_{+}\right)$associated with $\mathcal{G}$ whose convolution kernel is $\tilde{g}(\xi)$ by

$$
\left(\Gamma_{\mathcal{G}} u\right)(t)=\int_{0}^{\infty} \tilde{g}(t+\tau) u(\tau) d \tau
$$

If $u(t)=f_{-}(-t)$, then the Hankel operator gives the initial-condition response of the system, that is, the output of the system for time $t>0$ to the input of the system for time $t<0$. 
By Theorem 5.6.2 the adjoint of $\Gamma_{\mathcal{G}}$, denoted $\Gamma_{\mathcal{G}}^{*}$, is given by

$$
\left(\Gamma_{\mathcal{G}}^{*} u\right)(t)=\int_{0}^{\infty} \overline{\tilde{g}(t+\tau)} u(\tau) d \tau
$$

Hence, if $\tilde{g}$ is a real-valued function, $\Gamma_{\mathcal{G}}$ is a Hermitian operator. From Theorem 5.6.1 and Theorem 5.6.3, $\Gamma_{\mathcal{G}}$ is bounded if $\tilde{g} \in L_{1}\left(\mathbb{R}_{+}\right)$and $\Gamma_{\mathcal{G}}$ is compact if $\tilde{g} \in L_{2}\left(\mathbb{R}_{+}\right)$.

The $s$-domain representation of $\Gamma_{\mathcal{G}}$, denoted $\Gamma_{G}: H_{2}\left(\mathbb{C}^{+}\right) \rightarrow H_{2}\left(\mathbb{C}^{+}\right)$, is

$$
\left(\Gamma_{G} U\right)(s)=\Pi_{+}(G(s) U(-s))
$$

where $\Pi_{+}$is the orthogonal projection operator onto $H_{2}\left(\mathbb{C}^{+}\right), G(s)=\mathcal{L}\{\tilde{g}(t)\}$, and $U(-s)=\mathcal{L}\left\{f_{-}(t)\right\} . G(s)$ is called a symbol for $\Gamma_{G}$. For rational polynomials, $\Pi_{+} F(s)$ is calculated by obtaining a partial-fraction expansion for $F(s)$ and discarding the terms that are not analytic in $\mathbb{C}^{+}$. Other functions, in particular rational polynomials in a non-integer power, do not admit such an expansion. Consequently, there is no known means of calculating the orthogonal projection operator directly in $H_{2}\left(\mathbb{C}^{+}\right)$ when fractional-order systems are involved.

The representation of $\Gamma_{G}$ on $H_{2}(\mathbb{D})$, denoted $\Gamma_{g}: H_{2}(\mathbb{D}) \rightarrow H_{2}(\mathbb{D})$ is

$$
\left(\Gamma_{g} u\right)(z)=\Pi_{+}\left(g(z) u\left(z^{-1}\right)\right)
$$


where $\Pi_{+}$is the orthogonal projection operator onto $H_{2}(\mathbb{D})$, and $g(z)$ is given by

$$
g(z)=\frac{G\left(\frac{1-z}{1+z}\right)}{z} .
$$

$g(z)$ is called a symbol for $\Gamma_{g}$. Also note that $G(s)$ can be obtained from $g(z)$ using the mapping

$$
G(s)=g\left(\frac{1-s}{1+s}\right) \frac{1-s}{1+s} .
$$

For rational polynomials in $H_{2}(\mathbb{D}), \Pi_{+} f(z)$ is calculated by obtaining a partialfraction expansion for $f(z)$ and discarding the terms that are not analytic in $\mathbb{D}$. In general the functions $g(z) u\left(z^{-1}\right)$ do not admit a partial-fraction expansion when $g(z)$ is the symbol for a Hankel operator of a fractional-order system. Thus, there is no known means of calculating the orthogonal projection of such functions directly in $H_{2}(\mathbb{D})$.

Suppose that $g(z)$ has a Laurent series expansion given by

$$
g(z)=\sum_{k=-\infty}^{\infty} g_{k} z^{k} .
$$


The representation of $\Gamma_{g}$ on $\ell_{2}(\mathbb{N})$, denoted $\Gamma: \ell_{2}(\mathbb{N}) \rightarrow \ell_{2}(\mathbb{N})$, is given by

$$
\Gamma\left(\begin{array}{c}
u_{0} \\
u_{1} \\
u_{2} \\
\vdots
\end{array}\right)=\left(\begin{array}{cccc}
g_{0} & g_{1} & g_{2} & \cdots \\
g_{1} & g_{2} & g_{3} & \cdots \\
g_{2} & g_{3} & g_{4} & \cdots \\
\vdots & \vdots & \vdots & \ddots
\end{array}\right)\left(\begin{array}{c}
u_{0} \\
u_{1} \\
u_{2} \\
\vdots
\end{array}\right)
$$

which can be written as

$$
(\Gamma u)_{k}=\sum_{m=0}^{\infty} g_{m+k} u_{m} .
$$

The matrix in Equation 6.5 is called a Hankel matrix. The entries of a Hankel matrix depend only on the sum of the indices. The sequence $\left\{g_{k}\right\}$ is called a generating sequence for the Hankel operator $\Gamma$. Table 6.1 summarizes the notation we have developed.

Table 6.1: Hankel Operator Notation.

\begin{tabular}{lcc}
\hline \hline $\begin{array}{l}\text { Hankel } \\
\text { Operator }\end{array}$ & Domain & \multicolumn{2}{c}{$\begin{array}{c}\text { Kernel, Symbol, } \\
\text { or }\end{array}$} \\
\hline$\Gamma_{\mathcal{G}}$ & $L_{2}\left(\mathbb{R}_{+}\right)$ & $\tilde{g}(t)$ \\
$\Gamma_{G}$ & $H_{2}\left(\mathbb{C}^{+}\right)$ & $G(s)$ \\
$\Gamma_{g}$ & $H_{2}(\mathbb{D})$ & $g(z)$ \\
$\Gamma$ & $\ell_{2}(\mathbb{N})$ & $\left\{g_{k}\right\}$
\end{tabular}

Having defined equivalent Hankel operators on $L_{2}\left(\mathbb{R}_{+}\right), H_{2}(\mathbb{D}), H_{2}\left(\mathbb{C}^{+}\right)$, and $\ell_{2}(\mathbb{N})$, we now formally state that these operators are unitarily equivalent. 


\section{Theorem 6.1.1}

1. Let $\Gamma_{G}$ be a Hankel operator with symbol $G(s)$. Let

$$
g(z)=\frac{G\left(\frac{1-z}{1+z}\right)}{z} .
$$

Then $\Gamma_{g}$ and $\Gamma_{G}$ are unitarily equivalent operators.

2. Let $\Gamma_{g}$ be a Hankel operator with symbol $g(z)$. Let

$$
G(s)=g\left(\frac{1-s}{1+s}\right) \frac{1-s}{1+s} .
$$

Then $\Gamma_{G}$ and $\Gamma_{g}$ are unitarily equivalent operators.

3. Let $\Gamma_{G}$ be a Hankel operator with symbol $G(s)$. Let $\tilde{g}(t)=\mathcal{L}^{-1}\{G(s)\}$. Then $\Gamma_{\mathcal{G}}$ and $\Gamma_{G}$ are unitarily equivalent operators.

4. Let $\Gamma_{g}$ be a Hankel operator with symbol $g(z) . g(z)$ has a Laurent-series expansion

$$
g(z)=\sum_{k=-\infty}^{\infty} g_{k} z^{k}
$$


Define $\Gamma: \ell_{2}(\mathbb{N}) \rightarrow \ell_{2}(\mathbb{N})$ by

$$
\Gamma\left(\begin{array}{c}
u_{0} \\
u_{1} \\
u_{2} \\
\vdots
\end{array}\right)=\left(\begin{array}{cccc}
g_{0} & g_{1} & g_{2} & \cdots \\
g_{1} & g_{2} & g_{3} & \cdots \\
g_{2} & g_{3} & g_{4} & \cdots \\
\vdots & \vdots & \vdots & \ddots
\end{array}\right)\left(\begin{array}{c}
u_{0} \\
u_{1} \\
u_{2} \\
\vdots
\end{array}\right) .
$$

Then $\Gamma$ and $\Gamma_{g}$ are unitarily equivalent operators.

Proof See [73] and [75].

Because the operators $\Gamma_{\mathcal{G}}, \Gamma_{G}, \Gamma_{g}$, and $\Gamma$ are unitarily equivalent, henceforth we discuss the Hankel operators in the form that is most convenient for discussion.

\subsection{Well-Known Results Concerning Hankel Operators}

Nehari [60] gives a characterization of the bounded Hankel operators.

Theorem 6.2.1 (Nehari Theorem) The Hankel operator $\Gamma$ with generating sequence $\left\{g_{k}\right\}_{k=0}^{\infty}$ is bounded on $\ell_{2}(\mathbb{N})$ if and only if there exists a function $\phi \in L_{\infty}(\mathbb{T})$ with $\phi(z)=\sum_{-\infty}^{\infty} \phi_{k} z^{k}$ such that

$$
\phi_{k}=g_{k}, k \geq 0 .
$$


In this case,

$$
\|\Gamma\|=\inf \left\{\|\phi\|_{\infty}: \phi \in L_{\infty}(\mathbb{T}), \phi=\sum_{k=-\infty}^{\infty} \phi_{k} z^{k}, \text { and } \phi_{k}=g_{k} \text { for all } k \geq 0\right\}
$$

Proof See [60].

Because Hankel operators on $L_{2}\left(\mathbb{R}_{+}\right)$are integral operators whose kernels $\tilde{g}(t+\tau)$ have a special relationship with the impulse response $\tilde{g}(\xi)$, there is a condition on $\tilde{g}(\xi)$ which results in the Hankel operator being compact.

Theorem 6.2.2 Let $\tilde{g} \in L_{1}\left(\mathbb{R}_{+}\right)$. Then $\Gamma_{\mathcal{G}}: L_{2}\left(\mathbb{R}_{+}\right) \rightarrow L_{2}\left(\mathbb{R}_{+}\right)$is compact.

Proof See [75].

For Hankel operators on $L_{2}(\mathbb{T})$, there is a condition on $g(z)$ that is equivalent to $\Gamma_{g} \in \mathcal{B}_{0}\left(L_{2}(\mathbb{T})\right)$, but first we require two definitions.

Definition Define the set $C(E)=\{f: f$ is continuous on $E\}$. Also, define the set $C_{0}(i \mathbb{R})=\left\{F: F(s)\right.$ is continuous on $i \mathbb{R}$ and $\left.\lim _{|\omega| \rightarrow \infty}|F(i \omega)|=0\right\}$. Based on these definitions, $C_{0}(i \mathbb{R}) \subset C(i \mathbb{R})$.

Theorem 6.2.3 (Hartman's Theorem) The Hankel operator $\Gamma_{g}$ is compact if and only if $g \in H_{\infty}(\mathbb{D})^{\perp}+C(\mathbb{T})$.

Proof See [35]. 
The portion of a symbol $g(z)$ that is in $H_{\infty}(\mathbb{D})^{\perp}$ contributes nothing to the Hankel operator. Hartman's Theorem states that if the portion of $g(z)$ that is in $H_{\infty}(\mathbb{D})$ is also in $C(\mathbb{T})$, then the associated Hankel operator is compact. This condition for the compactness of $\Gamma_{g}$ can easily be converted to a condition for the compactness of $\Gamma_{G}$.

Corollary 6.2.4 $\Gamma_{G}$ is compact if and only if $G \in H_{\infty}\left(\mathbb{C}^{-}\right)+C_{0}(i \mathbb{R})$.

Proof See [73].

We will refer to both Theorem 6.2.3 and Corollary 6.2.4 as Hartman's theorem. Theorem 6.2.3 will be used in Section 6.5 to demonstrate the compactness of a class of Hankel operators.

A problem whose solution is relevant to the model-reduction problem and requires the spectral decomposition of the Hankel operator is the Nehari Problem. Nehari Problem Given a Hankel operator $\Gamma$ on $\ell_{2}(\mathbb{N})$ determine the best $n$-dimensional approximation, $\Gamma_{n}$ to $\Gamma$.

Recall that by the spectral theorem for compact Hermitian operators, Theorem 5.4.2, if $\Gamma$ is a compact operator, there exist $\left\{v_{n}\right\}$ and $\left\{w_{n}\right\}$ and scalars $\sigma_{n}$ decreasing to 0 such that

$$
\Gamma f=\sum_{k=1}^{\infty} \sigma_{k}\left\langle f, v_{k}\right\rangle w_{k}
$$


for all $f \in \mathcal{H}$. The singular values and Schmidt pairs prove quite useful in the solution of the Nehari problem. The Adamyan-Arov-Krein theorem [4], or AAK theorem, shows that this problem is well-posed, and gives a limit on how good of an approximation can be found.

Theorem 6.2.5 (Adamyan-Arov Krein Theorem) Let $\Gamma$ be a Hankel operator on $\ell_{2}(\mathbb{N})$ and let $n \geq 0$. Then there exists a Hankel operator $\Gamma_{n}: \ell_{2}(\mathbb{N}) \rightarrow \ell_{2}(\mathbb{N})$ of rank less than or equal to $n$ such that

$$
\left\|\Gamma-\Gamma_{n}\right\|=\sigma_{n+1}(\Gamma) .
$$

Proof See [4].

We now specifically consider Hankel operators on $L_{2}(\mathbb{R})$ for fractional-order systems and demonstrate that Hankel operators of non-integer rational-order systems are of infinite rank.

\subsection{Integral-Operator Hankel Operators for Fractional-Order Systems}

It has already been established in Section 3.4.2 that bounded fractional-order operators can be considered as convolution operators from $L_{2}(\mathbb{R})$ to $L_{2}(\mathbb{R})$. Then, let $\mathcal{G}: L_{2}(\mathbb{R}) \rightarrow L_{2}(\mathbb{R})$ be a fractional-order operator represented as a convolution 
operator, with impulse response $\tilde{g}(t)$ and transfer function $G(s)$. Then $\mathcal{G}$ is given by

$$
(\mathcal{G} u)(t)=\int_{-\infty}^{\infty} \tilde{g}(t-\tau) u(\tau) d \tau
$$

Since $u \in L_{2}(\mathbb{R})=L_{2}\left(\mathbb{R}_{-}\right) \oplus L_{2}\left(\mathbb{R}_{+}\right), \Pi_{-} u \in L_{2}\left(\mathbb{R}_{-}\right)$and $\Pi_{+} u \in L_{2}\left(\mathbb{R}_{+}\right)$. Define $u_{+}(t)=\Pi_{+} u(t)$ and $\hat{u}_{-}(t)=\Pi_{+} u(-t)$, noting that $\hat{u}_{-}(-t) \in L_{2}\left(\mathbb{R}_{+}\right)$and $\hat{u}_{-}(t)=\left.\left(\Pi_{-} u(\tau)\right)\right|_{\tau=-t}$. Then $\mathcal{G}$ can be written as

$$
\begin{aligned}
(\mathcal{G} u)(t) & =\int_{-\infty}^{\infty} \tilde{g}(t-\tau) u(\tau) d \tau=\int_{-\infty}^{0} \tilde{g}(t-\tau) u(\tau) d \tau+\int_{0}^{\infty} \tilde{g}(t-\tau) u(\tau) d \tau \\
& =-\int_{\infty}^{0} \tilde{g}(t+\tau) u(-\tau) d \tau+\int_{0}^{\infty} \tilde{g}(t-\tau) u(\tau) d \tau \\
& =\int_{0}^{\infty} \tilde{g}(t+\tau) \hat{u}_{-}(\tau) d \tau+\int_{0}^{\infty} \tilde{g}(t-\tau) u_{+}(\tau) d \tau \\
& =\left(\Gamma_{g} u\right)(t)+\int_{0}^{\infty} \tilde{g}(t-\tau) u_{+}(\tau) d \tau
\end{aligned}
$$

where $\Gamma_{g}: L_{2}\left(\mathbb{R}_{+}\right) \rightarrow L_{2}\left(\mathbb{R}_{+}\right)$is defined by

$$
\left(\Gamma_{g} u\right)(t)=\int_{0}^{\infty} \tilde{g}(t+\tau) \hat{u}_{-}(\tau) d \tau
$$

is a Hankel operator.

Recall Theorem 5.6.4, which says that for any measurable set $E$, an integral operator from $L_{2}(E)$ to $L_{2}(E)$ is finite rank if and only if its kernel is degenerate. Applied to integral operator representation of Hankel operators, this means that $\Gamma_{g}: L_{2}\left(\mathbb{R}_{+}\right) \rightarrow L_{2}\left(\mathbb{R}_{+}\right)$is finite rank if and only if $\tilde{g}(t+\tau)$ is degenerate; that is, 
there exist two finite sets of functions, $\left\{a_{k}(t)\right\}_{k=1}^{N}$ and $\left\{b_{k}(\tau)\right\}_{k=1}^{N}$ such that

$$
\tilde{g}(t+\tau)=\sum_{k=1}^{N} \alpha_{k}(t) \beta_{k}(\tau)
$$

It has already been shown in Section 5.6.3 that kernels for integer-order Hankel operators with simple poles are degenerate. It is noted that finite sums and finite products of degenerate kernels yield kernels that are also degenerate. Certain functional forms clearly give degenerate kernels. Kernels that are complex exponentials are degenerate. Kernels of the form $(\alpha t+\beta \tau)^{n}$ for $n \in \mathbb{N}$ are degenerate. Other functional forms obviously give non-degenerate kernels. Kernels of the form $(\alpha t+\beta \tau)^{q}$ for $q \in \mathbb{C} \backslash \mathbb{N}$ are non-degenerate. Kernels containing terms of the form $\gamma(\nu, a(t+\tau))$ for $\nu \in \mathbb{C} \backslash \mathbb{N}$ are non-degenerate because there do not exist two finite sets of functions, $\left\{a_{k}(t)\right\}_{k=1}^{N}$ and $\left\{b_{k}(\tau)\right\}_{k=1}^{N}$ such that

$$
\gamma(\nu, a(t+\tau))=\int_{0}^{a(t+\tau)} \xi^{\nu-1} e^{-\xi} d \xi=\sum_{k=1}^{N} \alpha_{k}(t) \beta_{k}(\tau)
$$

This leads to our next theorem, given in [5].

Theorem 6.3.1 Let $n \in \mathbb{N}$ be greater than one, let $\tilde{g}(t)$ be given by

$$
\tilde{g}(t)=\sum_{k=1}^{n} a^{k-1} E_{t}\left(\frac{k}{n}-1, a^{n}\right)
$$

and let $\Gamma_{g}: L_{2}\left(\mathbb{R}_{+}\right) \rightarrow L_{2}\left(\mathbb{R}_{+}\right)$be the Hankel operator associated with $\tilde{g}(t) . \Gamma_{g}$ is an infinite-rank operator. 
Proof Note that since $n>1, \tilde{g}(t)$ will always have the term, $a E_{t}\left(-\frac{n-1}{n}, a^{n}\right)$. Thus, $\tilde{g}(t+\tau)$ will have the term given by

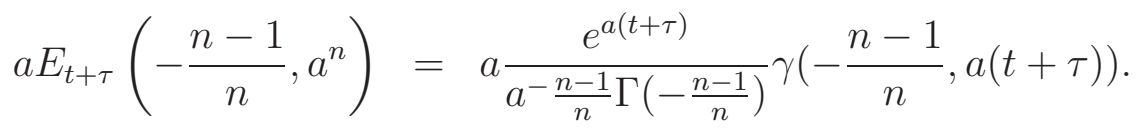

$\gamma\left(-\frac{n-1}{n}, a(t+\tau)\right)$ is non-degenerate, so the kernel given by $\tilde{g}(t+\tau)$ is non-degenerate.

Hence, by Theorem 5.6.4, $\Gamma_{g}$ is an infinite-rank operator.

Note from Section 3.4.3 that $\tilde{g}(t)$ is the impulse response for the system with transfer function

$$
G(s)=\frac{1}{s^{\frac{1}{n}}-a},
$$

so systems of this type have infinite-rank Hankel operators.

Recall that the Hankel operator maps the prior inputs of a system to its future outputs. The Hankel operator determines the initial condition response of a system. When a Hankel operator has infinite rank, its range is the span of an infinite number of distinct basis functions. An initial-condition response cannot be fully described with a finite number of initial conditions if the Hankel operator has infinite rank. That is, commensurate fractional-order systems are of infinite order, and as such, a finite number of initial conditions is not sufficient to properly account for initialization. Fractional-order systems require an infinite number of initial conditions to properly account for initialization of the system. 


\subsection{Hankel Operator Equivalences}

We will be considering Hankel operators with symbols of the form $\frac{r}{s^{q}-a}$. We now show that a Hankel operator whose symbol is of the form $\frac{r}{s^{q}-a}$ for $q \in \mathbb{C}^{-}$for $a \in \mathbb{C} \backslash\{0\}$ also has a symbol $\frac{\hat{r}}{s^{\hat{q}}-\hat{a}}$, where $\hat{q} \in \mathbb{C}^{+}$and $\hat{a} \in \mathbb{C} \backslash\{0\}$.

Theorem 6.4.1 Consider $G_{1}(s)$ and $G_{2}(s)$ the symbols for Hankel operators given by

$$
G_{1}(s)=\frac{r}{s^{-q}-a}
$$

and

$$
G_{2}(s)=-\frac{\frac{r}{a^{2}}}{s^{q}-\frac{1}{a}} .
$$

$G_{1}(s)$ and $G_{2}(s)$ give equivalent Hankel operators.

Proof Note that a symbol $g_{2}(z)$ for the operator $\Gamma_{g_{2}}$, which is unitarily equivalent to $\Gamma_{G_{2}}$, is given by

$$
g_{2}(z)=-\frac{1}{z} \frac{\frac{r}{a^{2}}}{\left(\frac{1-z}{1+z}\right)^{q}-\frac{1}{a}} .
$$

Note that

$$
G_{1}(s)=\frac{r}{s^{-q}-a}=-\frac{\frac{r}{a} s^{q}}{s^{q}-\frac{1}{a}}=-\frac{r}{a}-\frac{\frac{r}{a^{2}}}{s^{q}-\frac{1}{a}} .
$$

Consider the symbol $g_{1}(z)$ for the operator $\Gamma_{g_{1}}$, which is unitarily equivalent to $\Gamma_{G_{1}}$. $g_{1}(z)$ is given by

$$
g_{1}(z)=-\frac{r}{a} \frac{1}{z}-\frac{1}{z} \frac{\frac{r}{a^{2}}}{\left(\frac{1-z}{1+z}\right)^{q}-\frac{1}{a}} .
$$


Clearly, the first term in Equation 6.10 is not in $H_{2}(\mathbb{D})$, so an equivalent symbol $g_{1, e}(z)$ for $g_{1}(z)$ is

$$
g_{1, e}(z)=-\frac{1}{z} \frac{\frac{r}{a^{2}}}{\left(\frac{1-z}{1+z}\right)^{q}-\frac{1}{a}}=g_{2}(z) .
$$

Hence, $G_{1}(s)$ and $G_{2}(s)$ correspond to equivalent Hankel operators.

Corollary 6.4.2 The Hankel operators whose symbols are

$$
G_{1}(s)=\frac{r}{s^{-q}-a}+\frac{\bar{r}}{s^{-\bar{q}}-\bar{a}}
$$

and

$$
G_{2}(s)=-\frac{\frac{r}{a^{2}}}{s^{q}-\frac{1}{a}}-\frac{\frac{\overline{\bar{\sigma}}}{\overline{\bar{a}}^{2}}}{s^{\bar{q}}-\frac{1}{\bar{a}}}
$$

are equivalent.

Proof Since

$$
\begin{aligned}
G_{1}(s) & =\frac{r}{s^{-q}-a}+\frac{\bar{r}}{s^{-\bar{q}}-\bar{a}}=\frac{r s^{q}}{s^{q}-\frac{1}{a}}+\frac{\bar{r} s^{\bar{q}}}{s^{\bar{q}}-\frac{1}{\bar{a}}} \\
& =-\frac{r}{a}-\frac{\frac{r}{a^{2}}}{s^{q}-\frac{1}{a}}-\frac{\bar{r}}{\bar{a}} \frac{\frac{\bar{r}}{\bar{a}^{2}}}{s^{\bar{q}}-\frac{1}{\bar{a}}},
\end{aligned}
$$

the result follows in the same manner as the result of Theorem 6.4.1. 
6.5 Compactness of the Hankel Operator for a Class of Fractional-Order Systems

Before we may apply the algorithm given in Section 7.1 to an operator, we must demonstrate that the operator is compact. We will do so using Hartman's theorem, Theorem 6.2.3. Before we may apply Hartman's theorem, we need several well-known results on continuous functions.

Theorem 6.5.1 Let $f$ and $g$ be continuous functions from $X$ to $\mathbb{C}$ and let $\alpha, \beta \in \mathbb{C}$. Then $\alpha f+\beta g$ and $f g$ are both continuous. Also $f / g$ is continuous, provided $g(x) \neq 0$ for any $x \in X$.

Proof See [19].

Theorem 6.5.2 Let $f: X \rightarrow Y$ and $g: Y \rightarrow Z$ be continuous functions. Then $g \circ f$, where $(g \circ f)(x)=g(f(x))$, is a continuous function from $X$ to $Z$.

Proof See [19].

Suppose $G(s)=\frac{1}{s^{q}-a}$ with restrictions on $q$ and $a$ such that $G \in L_{\infty}(i \mathbb{R})$. Then Theorem 6.5.1 and Theorem 6.5.2 will be used to show $G(s) \in C(i \mathbb{R})$.

In order to show that $G(s) \in C(i \mathbb{R})$, we will apply Theorem 6.5.1 to $(i \omega)^{q}-a$. In order to do so, we need that $(i \omega)^{q}$ is continuous and that $(i \omega)^{q} \neq a$, which we prove in the next two lemmata. 
Lemma 6.5.3 Let $q \in \mathbb{C}^{+}$and let $f: \mathbb{R} \rightarrow \mathbb{C}$ be given by

$$
f(i \omega)=(i \omega)^{q}
$$

Then $f$ is continuous from $\mathbb{R}$ to $\mathbb{C}$.

Proof Let $u=\operatorname{Re}(q)$ and $v=\operatorname{Im}(q)$ and note that $u>0$. Then

$$
\begin{aligned}
f(i \omega) & =(i \omega)^{q}=\left(e^{i \frac{\pi}{2}} e^{\ln |\omega|+i \operatorname{Arg}(\omega)}\right)^{u+i v} \\
& =\left(e^{\ln |\omega|+i\left(\operatorname{Arg}(\omega)+\frac{\pi}{2}\right)}\right)^{u+i v} \\
& =e^{u \ln |\omega|-v\left(\operatorname{Arg}(\omega)+\frac{\pi}{2}\right)} e^{i\left(v \ln |\omega|+u\left(\operatorname{Arg}(\omega)+\frac{\pi}{2}\right)\right)} .
\end{aligned}
$$

Since $\omega \in \mathbb{R}, \operatorname{Arg}(\omega)$ can be written as

$$
\operatorname{Arg}(\omega)=\frac{\pi}{2}(1-\operatorname{sgn}(\omega))
$$

Substituting Equation 6.12 into Equation 6.11 results in

$$
f(i \omega)=e^{u \ln |\omega|-v\left(\pi\left(1-\frac{\operatorname{sgn}(\omega)}{2}\right)\right)} e^{i\left(v \ln |\omega|+u\left(\pi\left(1-\frac{\operatorname{sgn}(\omega)}{2}\right)\right)\right)} .
$$

Note that $\ln |\omega|$ and $\operatorname{sgn}(\omega)$ are continuous on $(-\infty, 0)$ and on $(0, \infty)$ and that $e^{z}$ is continuous for all $z \in \mathbb{C}$. By Theorem 6.5.1 and Theorem 6.5.2, $f$ is continuous on $(-\infty, 0)$ and on $(0, \infty)$ 
We now show that $f$ is continuous at $\omega=0$. Note that

$$
\begin{aligned}
|f(i \omega)| & =\left|e^{u \ln |\omega|-v\left(\pi\left(1-\frac{\operatorname{sgn}(\omega)}{2}\right)\right)} e^{i\left(v \ln |\omega|+u\left(\pi\left(1-\frac{\operatorname{sgn}(\omega)}{2}\right)\right)\right)}\right| \\
& =\left|e^{u \ln |\omega|-v\left(\pi\left(1-\frac{\operatorname{sgn}(\omega)}{2}\right)\right)}\right| \\
& =e^{u \ln |\omega|-v\left(\pi\left(1-\frac{\operatorname{sgn}(\omega)}{2}\right)\right)} \\
& =|\omega|^{u} e^{-v\left(\pi\left(1-\frac{\operatorname{sgn}(\omega)}{2}\right)\right)}
\end{aligned}
$$

Note that $f(i 0)=0$. Let $\epsilon>0$ be given. Let $\delta>0$ be given by

$$
\delta=\min \left\{\left(\frac{\epsilon}{2}\right)^{\frac{1}{u}} e^{\frac{\pi v}{2 u}},\left(\frac{\epsilon}{2}\right)^{\frac{1}{u}} e^{3 \frac{\pi v}{2 u}}\right\} .
$$

For $\omega>0$ such that $|\omega|<\delta$, since $u>0$,

$$
\begin{aligned}
|f(i \omega)| & =|\omega|^{u} e^{-v\left(\pi\left(1-\frac{\operatorname{sgn}(\omega)}{2}\right)\right)} \\
& \leq\left|\left(\frac{\epsilon}{2}\right)^{\frac{1}{u}} e^{3 \frac{\pi v}{2 u}}\right|^{u} e^{-v \frac{3}{2} \pi} \\
& =\frac{\epsilon}{2} e^{3 \frac{\pi v}{2}} e^{-v \frac{3}{2} \pi}=\frac{\epsilon}{2}<\epsilon .
\end{aligned}
$$

Thus, $\lim _{\omega \rightarrow 0^{+}} f(i \omega)=0$. Similarly, for $\omega<0$ such that $|\omega|<\delta$,

$$
\begin{aligned}
|f(i \omega)| & =|\omega|^{u} e^{-v\left(\pi\left(1-\frac{\operatorname{sgn}(\omega)}{2}\right)\right)} \\
& \leq\left|\left(\frac{\epsilon}{2}\right)^{\frac{1}{u}} e^{\frac{\pi v}{2 u}}\right|^{u} e^{-\frac{v}{2} \pi} \\
& =\frac{\epsilon}{2} e^{\frac{\pi v}{2}} e^{-\frac{v}{2} \pi}=\frac{\epsilon}{2}<\epsilon .
\end{aligned}
$$


Thus, $\lim _{\omega \rightarrow 0^{-}} f(i \omega)=0$, and subsequently, $\lim _{\omega \rightarrow 0} f(i \omega)=0=f(i 0)$. Hence, $f$ is continuous at 0 .

$f$ is thus continuous on $(-\infty, \infty)$.

Lemma 6.5.4 Given $a \in \mathbb{C}$ and $q \in \mathbb{C}^{+}$such that $a \neq 0$ and

$$
\arg (a) \neq \frac{\operatorname{Im}(q)}{\operatorname{Re}(q)}\left(\ln |a| \pm \operatorname{Im}(q) \frac{\pi}{2}\right) \pm \operatorname{Re}(q) \frac{\pi}{2}
$$

Then $(i \omega)^{q} \neq$ a for any $\omega \in \mathbb{R}$.

Proof Let $a \in \mathbb{C}$ and $q \in \mathbb{C}^{+}$satisfy Equation 6.15. Suppose that there exists an $\omega_{0} \in \mathbb{R}$ such that $\left(i \omega_{0}\right)^{q}=a$. Let $u=\operatorname{Re}(q)$ and $v=\operatorname{Im}(q)$. Then,

$$
\begin{aligned}
|a| e^{i \arg (a)}=a & =\left(i \omega_{0}\right)^{q}=\left(e^{ \pm i \frac{\pi}{2}} e^{\ln \left|\omega_{0}\right|}\right)^{u+i v} \\
& =e^{u \ln \left|\omega_{0}\right| \mp v \frac{\pi}{2}} e^{i\left(v \ln \left|\omega_{0}\right| \pm u \frac{\pi}{2}\right)}
\end{aligned}
$$

Hence,

$$
|a|=e^{u \ln \left|\omega_{0}\right| \mp v \frac{\pi}{2}}
$$

and

$$
\arg (a)=v \ln \left|\omega_{0}\right| \pm u \frac{\pi}{2}
$$


We solve Equation 6.16 for $\ln \left|\omega_{0}\right|$ to find

$$
\ln \left|\omega_{0}\right|=\frac{\ln |a|}{u} \pm\left(\frac{v}{u}\right) \frac{\pi}{2}
$$

Substituting Equation 6.18 into Equation 6.17 results in

$$
\arg (a)=\frac{v}{u}\left(\ln |a| \pm v \frac{\pi}{2}\right) \pm u \frac{\pi}{2}
$$

which contradicts Equation 6.15. Hence, $(i \omega)^{q} \neq a$ for any $\omega \in \mathbb{R}$.

Lemma 6.5.5 will be useful in proving $\Gamma_{G}$ compact.

Lemma 6.5.5 $a \in \mathbb{C} \backslash\{0\}$ and $q \in \mathbb{C}^{+}$satisfies

$$
\arg (a)=\frac{\operatorname{Im}(q)}{\operatorname{Re}(q)}\left(\ln |a| \pm \operatorname{Im}(q) \frac{\pi}{2}\right) \pm \operatorname{Re}(q) \frac{\pi}{2}
$$

if and only if a and $q$ satisfy

$$
\arg \left(\frac{1}{a}\right)=\frac{\operatorname{Im}(q)}{\operatorname{Re}(q)}\left(\ln \left|\frac{1}{a}\right| \pm \operatorname{Im}(q) \frac{\pi}{2}\right) \pm \operatorname{Re}(q) \frac{\pi}{2}
$$

Also, Equation 6.20 is satisfied if and only if

$$
\arg (\bar{a})=\frac{-\operatorname{Im}(q)}{\operatorname{Re}(q)}\left(\ln |\bar{a}| \pm(-\operatorname{Im}(q)) \frac{\pi}{2}\right) \pm \operatorname{Re}(q) \frac{\pi}{2}
$$


Proof Note that the two values of $\frac{\operatorname{Im}(q)}{\operatorname{Re}(q)}\left(\ln |a| \pm \operatorname{Im}(q) \frac{\pi}{2}\right) \pm \operatorname{Re}(q) \frac{\pi}{2}$ are the same two quantities as $\frac{\operatorname{Im}(q)}{\operatorname{Re}(q)}\left(\ln |a| \mp \operatorname{Im}(q) \frac{\pi}{2}\right) \mp \operatorname{Re}(q) \frac{\pi}{2}$. Equation 6.20 is satisfied if and only if

$$
\begin{aligned}
\arg \left(\frac{1}{a}\right) & =-\arg (a)=-\frac{\operatorname{Im}(q)}{\operatorname{Re}(q)}\left(\ln |a| \pm \operatorname{Im}(q) \frac{\pi}{2}\right) \mp \operatorname{Re}(q) \frac{\pi}{2} \\
& =\frac{\operatorname{Im}(q)}{\operatorname{Re}(q)}\left(-\ln |a| \mp \operatorname{Im}(q) \frac{\pi}{2}\right) \mp \operatorname{Re}(q) \frac{\pi}{2} \\
& =\frac{\operatorname{Im}(q)}{\operatorname{Re}(q)}\left(\ln \left|\frac{1}{a}\right| \mp \operatorname{Im}(q) \frac{\pi}{2}\right) \mp \operatorname{Re}(q) \frac{\pi}{2},
\end{aligned}
$$

which proves the first part of the lemma. Equation 6.20 is satisfied if and only if

$$
\begin{aligned}
\arg (\bar{a}) & =-\arg (a)=-\frac{\operatorname{Im}(q)}{\operatorname{Re}(q)}\left(\ln |a| \pm \operatorname{Im}(q) \frac{\pi}{2}\right) \mp \operatorname{Re}(q) \frac{\pi}{2} \\
& =\frac{-\operatorname{Im}(q)}{\operatorname{Re}(q)}\left(\ln |\bar{a}| \mp(-\operatorname{Im}(q)) \frac{\pi}{2}\right) \mp \operatorname{Re}(q) \frac{\pi}{2},
\end{aligned}
$$

which proves the second part of the lemma.

We now show that $\Gamma_{G}$ is a compact operator.

Theorem 6.5.6 Suppose that $G(s)=\frac{1}{s^{q}-a}$ with $q \in \mathbb{C} \backslash i \mathbb{R}$ and $a \in \mathbb{C}$ such that $a \neq 0$ and

$$
\arg (a) \neq \frac{\operatorname{Im}(q)}{\operatorname{Re}(q)}\left(\ln |a| \pm \operatorname{Im}(q) \frac{\pi}{2}\right) \pm \operatorname{Re}(q) \frac{\pi}{2}
$$

Then $\Gamma_{G}$ is compact.

Proof We will prove that $G(s) \in C_{0}(i \mathbb{R})$ so we may apply Hartman's theorem. 
Suppose that $q \in \mathbb{C}^{-}$. Then by Theorem 6.4.1, $\Gamma_{G}$ also has symbol $\hat{G}(s)=$ $\frac{\frac{1}{a^{2}}}{s^{-q}-\frac{1}{a}}$, and by Lemma 6.5.5 this symbol satisfies the condition in Equation 6.23 for $\hat{q}=-q$ and $\hat{a}=\frac{1}{a}$. Thus, without loss of generality, we may assume that $q \in \mathbb{C}^{+}$.

By Lemma 6.5.3, $(i \omega)^{q}$ is continuous from $\mathbb{R}$ to $\mathbb{C}$. By Theorem 6.5.1, $(i \omega)^{q}-a$ is continuous for $a \in \mathbb{C}$ since $a$ is continuous. By Lemma 6.5.4, $(i \omega)^{q} \neq a$ for any $\omega \in \mathbb{R}$, so $(i \omega)^{q}-a \neq 0$ for any $\omega \in \mathbb{R}$. Thus, by Theorem $6.5 .1, G(s)=\frac{1}{(i \omega)^{q}-a}$ is continuous because 1 and $(i \omega)^{q}-a$ are continuous with $(i \omega)^{q}-a \neq 0$ for all $\omega \in \mathbb{R}$. Note that $\lim _{|\omega| \rightarrow \infty}|G(i \omega)|=0$ Hence, $G(s) \in C_{0}(i \mathbb{R}) \subset C(i \mathbb{R})$, and subsequently, by Hartman's theorem, $\Gamma_{G}$ is compact.

Corollary 6.5.7 Suppose that $G(s)=\frac{r}{s^{q}-a}+\frac{\bar{r}}{s^{\bar{q}}-\bar{a}}$ with $q \in \mathbb{C} \backslash i \mathbb{R}$ and $a, r \in \mathbb{C}$ such that $a \neq 0$ and

$$
\arg (a) \neq \frac{\operatorname{Im}(q)}{\operatorname{Re}(q)}\left(\ln |a| \pm \operatorname{Im}(q) \frac{\pi}{2}\right) \pm \operatorname{Re}(q) \frac{\pi}{2}
$$

Then $\Gamma_{G}$ is compact.

Proof Note that $G_{1}(s)=\frac{r}{s^{q}-a}$ and $G_{2}(s)=\frac{\bar{r}}{s^{\bar{q}}-\bar{a}}$ satisfy the conditions of Theorem 6.5.6, using Lemma 6.5.5 for $G_{2}(s)$. Thus, as shown in the proof of Theorem 6.5.6, $G_{1}(s), G_{2}(s) \in C(i \mathbb{R})$. By Theorem 6.5.1, $\left(G_{1}(s)+G_{2}(s)\right) \in C(i \mathbb{R})$. Further, $\lim _{|\omega| \rightarrow \infty}|G(i \omega)|=0$. Subsequently, by Hartman's theorem, $\Gamma_{G}$ is compact. 
If $q$ is real then the condition as in Equation 6.23 is simpler. We state this formally.

Corollary 6.5.8 Suppose that $G(s)=\frac{1}{s^{q}-a}$ with $q \in(0, \infty)$ and $a \in \mathbb{C}$ such that $a \neq 0$ and

$$
\arg a \neq \pm q \frac{\pi}{2}
$$

Then $\Gamma_{G}$ is compact.

Proof If $q \in(0, \infty)$, then $\operatorname{Re}(q)=q>0$ and $\operatorname{Im}(q)=0$. $a$ satisfies the condition of Equation 6.23, and Theorem 6.5.6 is applied to give the result.

As a final corollary to Theorem 6.5.6, we demonstrate the compactness of a class of operators, whose Hankel singular values we will estimate in Section 7.2.3.

Corollary 6.5.9 Let $G(s)=\frac{1}{s^{q}+1}$ where $q \in(0, \infty)$ such that $q \neq 4 k+2$ for any $k \in \mathbb{N}$. Then $\Gamma_{G}$ is a compact operator.

Proof Let $q \in(0, \infty)$ such that $q \neq 2(2 k+1)$ for any $k \in \mathbb{N}$. Suppose that $\arg (-1)=q \frac{\pi}{2}$ or $\arg (-1)=-q \frac{\pi}{2}$. Note that $\arg (-1)=(2 k+1) \pi$ for some $k \in \mathbb{Z}$. Hence, either

$$
q=\frac{2}{\pi} \arg (-1)=\frac{2}{\pi}(2 k+1) \pi=2(2 k+1)
$$


for some $k \in \mathbb{Z}$, which contradicts either $q \in(0, \infty)$ or $q \neq 2(2 k+1)$, or

$$
q=-\frac{2}{\pi} \arg (-1)=-\frac{2}{\pi}(2 k+1) \pi=-2(2 k+1)
$$

for some $k \in \mathbb{Z}$, which contradicts either $q \in(0, \infty)$ or $q \neq 2(2 k+1)$. Thus, Equation 6.25 is satisfied. By Corollary 6.5.8, $\Gamma_{G}$ is compact.

We next demonstrate the conditions under which the Hankel operator for the fundamental, linear conjugate-order system is unbounded.

Theorem 6.5.10 Suppose that $G(s)=\frac{r}{s^{q}-a}+\frac{\bar{r}}{s^{\bar{q}}-\bar{a}}$ with $q \in \mathbb{C} \backslash i \mathbb{R}$ and $a, r \in \mathbb{C}$ such that $a \neq 0$ and

$$
\arg (a)=\frac{\operatorname{Im}(q)}{\operatorname{Re}(q)}\left(\ln |a| \pm \operatorname{Im}(q) \frac{\pi}{2}\right) \pm \operatorname{Re}(q) \frac{\pi}{2}
$$

Then $\Gamma_{G}$ is unbounded.

Proof Note that by Nehari's theorem, $\Gamma_{g}$ is bounded if and only if there exists a symbol $g(z)$ of $\Gamma_{g}$ such that $g \in H_{\infty}(\mathbb{D})$. An equivalent statement is that $\Gamma_{G}$ is bounded if and only if there exists a symbol $G(s)$ of $\Gamma_{G}$ such that $G \in H_{\infty}\left(\mathbb{C}^{+}\right)$.

Define $u=\operatorname{Re}(q)$ and $v=\operatorname{Im}(q)$. Suppose $G \in H_{\infty}\left(\mathbb{C}^{+}\right)$, then $G \in L_{\infty}(i \mathbb{R})$, so there exists an $M \in(0, \infty)$ such that $G(i \omega) \leq M$ for all $\omega \in \mathbb{R}$. This can be 
rewritten as

$$
\frac{1}{G(i \omega)}=\frac{\left((i \omega)^{q}-a\right)\left((i \omega)^{\bar{q}}-\bar{a}\right)}{r\left((i \omega)^{\bar{q}}-\bar{a}\right)+\bar{r}\left((i \omega)^{q}-a\right)} \geq \frac{1}{M}>0
$$

for all $\omega \in \mathbb{R}$.

Then, by Equation 6.26,

$$
\begin{aligned}
\arg \left(a^{\frac{1}{q}}\right) & =\arg \left(e^{\frac{\ln |a|+i \arg (a)}{u+i v}}\right) \\
& =\arg \left(e^{\frac{(\ln |a|+i \arg (a))(u-i v)}{u^{2}+v^{2}}}\right) \\
& =\arg \left(e^{\frac{u \ln |a|+v \arg (a)+i(u \arg (a)-v \ln |a|)}{u^{2}+v^{2}}}\right) \\
& =\frac{u \arg (a)-v \ln |a|}{u^{2}+v^{2}} \\
& =\frac{u \frac{v}{u}\left(\ln |a| \pm v \frac{\pi}{2}\right) \pm u \frac{\pi}{2}-v \ln |a|}{u^{2}+v^{2}} \\
& =\frac{v \ln |a| \pm v^{2} \frac{\pi}{2} \pm u^{2} \frac{\pi}{2}-v \ln |a|}{u^{2}+v^{2}} \\
& = \pm \frac{\pi}{2} .
\end{aligned}
$$

Hence, $-i(a)^{\frac{1}{q}} \in \mathbb{R}$. Choose $\omega_{0}=-i(a)^{\frac{1}{q}}$. Then

$$
\begin{aligned}
\left(i \omega_{0}\right)^{q}-a & =\left(i\left(-i(a)^{\frac{1}{q}}\right)\right)^{q}-a \\
& =a^{\frac{q}{q}}-a=a-a=0<\frac{1}{M}
\end{aligned}
$$

Therefore $G \notin L_{\infty}(i \mathbb{R})$, so $G \notin H_{\infty}\left(\mathbb{C}^{+}\right)$, and $\Gamma_{G}$ is unbounded. 
Corollary 6.5.11 Suppose that $G(s)=\frac{r}{s^{q}-a}+\frac{\bar{r}}{s^{\bar{q}}-\bar{a}}$ with $q \in \mathbb{C} \backslash i \mathbb{R}$ and $a, r \in \mathbb{C}$ such that $a \neq 0$. If $\Gamma_{G}$ is not compact, then $\Gamma_{G}$ is unbounded.

Proof $a$ and $q$ satisfy either Equation 6.24 or Equation 6.26. If $a$ and $q$ satisfy the former, then, by Corollary 6.5.7, $\Gamma_{G}$ is compact. Otherwise, by Theorem 6.5.10, $\Gamma_{G}$ is unbounded.

Recall the regions in the $(u, v)$ plane for a fixed $a$ in Section 4.4 .1 and the regions in the $(\ln |a|, \arg (a))$ plane for fixed $q$ in Section 4.4.3 such that $G(s) \frac{r}{s^{q}-a}+\frac{\bar{r}}{s^{\bar{q}}-\bar{a}}$ corresponds to a stable, causal system. The borders of these regions correspond to the $q$ or $a$ for which $\Gamma_{G}$ is an unbounded operator. The regions correspond to he $q$ or $a$ for which $\Gamma_{G}$ is a nonzero, compact operator. Finally, the exterior of the regions and the borders correspond to the $q$ or $a$ for which $\Gamma_{G}$ is zero.

We also show that Hankel operators with symbol $G_{1}(s)=\frac{r}{s^{q}-a}+\frac{\bar{r}}{s^{\bar{q}}-\bar{a}}$ are Hermitian.

Theorem 6.5.12 The Hankel operator with symbol

$$
G(s)=\frac{r}{s^{q}-a}+\frac{\bar{r}}{s^{\bar{q}}-\bar{a}}
$$

is Hermitian.

Proof Let $\tilde{g}(t)$ be the impulse response of $G(s)$. Note that by Theorem 6.1.1 $\Gamma_{G}$ and $\Gamma_{\mathcal{G}}$ are unitarily equivalent. Thus, $\Gamma_{G}$ is Hermitian if and only if $\Gamma_{\mathcal{G}}$ is Hermi- 
tian. Since $\tilde{g}(t)$ is a real-valued function by Theorem 4.3.1, by Theorem 5.6.2, $\Gamma_{\mathcal{G}}$ is Hermitian.

\subsection{Examples}

We now consider the Hankel operators for the second-order system, as well as the resistor-terminated and inductor-terminated semi-infinite lines.

\subsubsection{Second-Order Example}

Recall the second order system introduced in Section 2.4.1, whose transfer function is

$$
G(s)=\frac{-2 s}{s^{2}+s+\frac{1}{4}}
$$

and whose impulse response is

$$
\tilde{g}(t)=e^{-\frac{t}{2}}(t-2) \mathcal{U}_{s}(t)
$$

Note that $G(s)$ is a symbol for $\Gamma_{G}: L_{2}(i \mathbb{R}) \rightarrow L_{2}(i \mathbb{R})$. We showed that $G(s) \in$ $L_{\infty}(i \mathbb{R})$ in Section 5.8.1, and it is easily shown that $G(s) \in C(i \mathbb{R})$. Hence, by Hartman's theorem, $\Gamma_{G}$ is compact. A symbol for the unitarily equivalent Hankel 
operator $\Gamma_{g}: H_{2}(\mathbb{D}) \rightarrow H_{2}(\mathbb{D})$ is

$$
\begin{aligned}
g(z) & =\Pi_{+} \frac{1}{z} G\left(\frac{1+z}{1-z}\right)=\Pi_{+}\left(\frac{8\left(z^{2}-1\right)}{z(z-3)^{2}}\right) \\
& =\frac{16(5 z-3)}{9(z-3)^{2}} .
\end{aligned}
$$

Since $g(z) \in C(\mathbb{T})$, by Hartman's theorem, $\Gamma_{g}$ is compact. In fact, since $\Gamma_{g}$ is unitarily equivalent to $\Gamma_{G}$ and $\Gamma_{G}$ is compact, the compactness of $\Gamma_{g}$ follows. Note that $g(z) \in$ $L_{\infty}(\mathbb{T})$ and that

$$
g(z)=-\frac{16}{27}+\frac{16}{27} z+\frac{112}{243} z^{2}+\frac{176}{729} z^{3}+\frac{80}{729} z^{4}+\frac{304}{6561} z^{5}+\frac{368}{19683} z^{6}+\cdots
$$

The generating sequence for the unitarily equivalent operator $\Gamma: \ell_{2}(\mathbb{N}) \rightarrow \ell_{2}(\mathbb{N})$ is

$$
\left\{g_{k}\right\}=\left\{-\frac{16}{27}, \frac{16}{27}, \frac{112}{243}, \frac{176}{729}, \frac{80}{729}, \frac{304}{6561}, \frac{368}{19683}, \cdots\right\}
$$

Because $g(z) \in L_{\infty}(\mathbb{T})$, by Nehari's theorem, $\Gamma_{g}$ is bounded. $\Gamma_{g}$ is compact because it is unitarily equivalent to $\Gamma_{G}$ and $\Gamma_{g}$, compact operators. The representation for the Hankel operator $\Gamma_{\mathcal{G}}$ on $L_{2}\left(\mathbb{R}_{+}\right)$is

$$
\left(\Gamma_{\mathcal{G}} f\right)(t)=\int_{0}^{\infty} e^{-\frac{t+\tau}{2}}(t+\tau-2) f_{-}(\tau) d \tau
$$

for $t>0 . \tilde{g}(t) \in L_{1}\left(\mathbb{R}_{+}\right)$, so by Theorem $6.2 .2, \Gamma_{\mathcal{G}}$ is compact. It was demonstrated in Section 5.8.1 that this is a rank-two operator with two singular values of unity. 


\subsubsection{Resistor-Terminated Semi-Infinite Line}

Recall that the resistor-terminated semi-infinite line has transfer function

$$
G_{R}(s)=\frac{1}{s^{\frac{1}{2}}+1}
$$

and impulse response

$$
\tilde{g}_{R}(t)=\sum_{k=1}^{2}(-1)^{k-1} E_{t}\left(\frac{k-2}{2}, 1\right) .
$$

A symbol for $\Gamma_{G}$ is $G_{R}(s)$. Since $G_{R}(s) \in C(i \mathbb{R})$, by Hartman's theorem $\Gamma_{G}$ is compact. A symbol for the unitarily equivalent Hankel operator $\Gamma_{g}: H_{2}(\mathbb{D}) \rightarrow H_{2}(\mathbb{D})$ is

$$
g_{R}(z)=\Pi_{+}\left(\frac{G_{R}\left(\frac{1+z}{1-z}\right)}{z}\right)=\frac{1}{z\left(\sqrt{\frac{1+z}{1-z}}+1\right)}-\frac{1}{2 z},
$$

which is in $C(\mathbb{T})$. Again, by Hartman's theorem, $\Gamma_{g}$ is compact. Using the Laurent series expansion for $g_{R}(z)$, we obtain a generating sequence for the unitarily equivalent Hankel operator $\Gamma: \ell_{2}(\mathbb{N}) \rightarrow \ell_{2}(\mathbb{N})$ as

$$
\left\{g_{R, k}\right\}=\left\{\frac{1}{4}, 0, \frac{1}{16}, 0, \frac{1}{32}, 0, \frac{5}{256}, 0, \frac{7}{512}, \cdots\right\}
$$


Because $g_{R}(z) \in L_{\infty}(i \mathbb{R})$, by Nehari's theorem $\Gamma$ is bounded. The representation of $\Gamma_{\mathcal{G}}: L_{2}\left(\mathbb{R}_{+}\right) \rightarrow L_{2}\left(\mathbb{R}_{+}\right)$is

$$
\left(\Gamma_{\mathcal{G}} f\right)(t)=\int_{0}^{\infty} \sum_{k=1}^{2}(-1)^{k-1} E_{t+\tau}\left(\frac{k-2}{2}, 1\right) f(\tau) d \tau .
$$

It was shown in [8] that $\tilde{g}_{R}(t) \in L_{1}\left(\mathbb{R}_{+}\right)$using the representation of Miller and Ross for $\tilde{g}_{R}(t)$. It follows that $\Gamma_{\mathcal{G}}$ is compact.

The discussion in Section 6.3 applies to this Hankel operator, and $\Gamma_{G}$ is an

infinite-rank operator. Note that $\frac{1}{2} \neq 4 k+2$ for any $k \in \mathbb{Z}$. Application of Corollary 6.5.9 and Theorem 6.5.12 give that $\Gamma_{G}$ is a compact, Hermitian operator. Recall that $\mathcal{G}_{R}$ is not Hermitian. The results for this Hankel operator are with one exception the same as those obtained for the second-order system. Both Hankel operators are bounded, compact, and Hermitian. The difference is that the Hankel operator for the second-order system is finite-rank while the Hankel operator for the resistorterminated semi-infinite line is infinite-rank.

\subsubsection{Inductor-Terminated Semi-Infinite Line}

Recall that the inductor-terminated semi-infinite line has transfer function

$$
G_{L}(s)=\frac{1}{s^{\frac{3}{2}}+1}
$$

and impulse response

$$
\tilde{g}_{L}(t)=F_{\frac{3}{2}}(1, t) .
$$


A symbol for $\Gamma_{G}$ is $G_{L}(s)$. By Hartman's theorem, since $G_{L}(s) \in C(i \mathbb{R}), \Gamma_{G}$ is compact. A symbol for the unitarily equivalent Hankel operator $\Gamma_{g}: H_{2}(\mathbb{D}) \rightarrow H_{2}(\mathbb{D})$ is

$$
g_{L}(z)=\Pi_{+}\left(\frac{G_{L}\left(\frac{1+z}{1-z}\right)}{z}\right)=\frac{1}{z\left(\left(\frac{1+z}{1-z}\right)^{\frac{3}{2}}+1\right)}-\frac{1}{2 z} .
$$

Because $g_{L}(z) \in C(\mathbb{T}), \Gamma_{g}$ is compact. Using the Laurent series expansion for $g_{L}(z)$, we obtain a generating sequence for $\Gamma: \ell_{2}(\mathbb{N}) \rightarrow L_{2}\left(\mathbb{R}_{+}\right)$as

$$
\left\{g_{L, k}\right\}=\left\{\frac{3}{4}, 0,-\frac{5}{16}, 0, \frac{3}{32}, 0,-\frac{9}{256}, 0, \frac{5}{512}, \cdots\right\} .
$$

Because $g_{L}(z) \in L_{\infty}(\mathbb{T})$, by Nehari's theorem $\Gamma$ is bounded. The representation of $\Gamma_{\mathcal{G}}$ is

$$
\left(\Gamma_{\mathcal{G}} f\right)(t)=\int_{0}^{\infty} F_{\frac{3}{2}}(1, t+\tau) f(\tau) d \tau
$$

Because the sum in the definition of the $F$-function is infinite, it is more difficult to determine whether $\tilde{g}_{L}(t)$ is integrable or not. However, because $\Gamma$ is unitarily equivalent to a compact Hankel operator $\Gamma_{g}, \Gamma$ is itself compact.

This Hankel operator is an infinite-rank operator as per the discussion in Section 6.3. Note that $\frac{3}{2} \neq 4 k+2$ for any $k \in \mathbb{Z}$. Application of Corollary 6.5.9 and Theorem 6.5.12 give that $\Gamma_{G}$ is a compact, Hermitian operator. Like the resistorterminated semi-finite line, the inductor-terminated line has a Hankel operator that is bounded, compact, Hermitian, and infinite-rank. 


\subsection{Conclusions}

We have discussed four representations of Hankel operators on four isomorphic Hilbert spaces. Conditions under which a Hankel operator is bounded or compact are discussed. We have related the Hankel operator of a fundamental, linear conjugate-order system of order $q$ with the Hankel operator of another fundamental, linear complex order system with order $-q$. Sufficient conditions under which the fundamental, linear conjugate-order system has a compact Hankel operator were established. We now present an algorithm by which the Hankel singular values of a fractional-order system may be approximated. 


\section{CHAPTER VII}

\section{ESTIMATING THE HANKEL SINGULAR VALUES OF FRACTIONAL-ORDER SYSTEMS}

A method to approximate the Hankel singular values of a fractional-order system is given. This algorithm improves on the method given in [8] by also providing upper bounds on the Hankel singular values. The algorithm is demonstrated on a secondorder system as an example. The algorithm is then applied to a class of fractionalorder operators of the form $G(s)=\frac{a}{s^{\frac{1}{2}}+a}$. Finally, the algorithm is applied to a class of fractional-order operators of the form $G(s)=\frac{1}{s^{q}+1}$.

\subsection{Methodology}

The method we use to determine bounded estimates of the Hankel singular values for fractional-order systems is an application of the Rayleigh-Ritz method for eigenvalue approximation given in Section 5.7. This method is only applicable for compact, Hermitian operators and utilizes the equivalence of Hankel operators in different Hilbert spaces. Recall that $H_{2}\left(\mathbb{C}^{+}\right) \cong L_{2}\left(\mathbb{R}_{+}\right) \cong H_{2}(\mathbb{D}) \cong \ell_{2}(\mathbb{N})$ and that there are unitarily equivalent representations for the Hankel operator on each of the isomorphic Hilbert spaces, given in Theorem 6.1.1. Because it is convenient to describe a system 
by its transfer function, we assume that the fractional-order system whose Hankel singular values we wish to determine is given as a transfer function $G(s) \in L_{\infty}(i \mathbb{R})$.

The following terminology is used throughout the description of the algorithm. $G(s)$ is the transfer function for the system. $G(s)$ is also a symbol for the Hankel operator, $\Gamma_{G}: H_{2}\left(\mathbb{C}^{+}\right) \rightarrow H_{2}\left(\mathbb{C}^{+}\right) . g(z)$ is a symbol for the Hankel operator $\Gamma_{g}: H_{2}(\mathbb{D}) \rightarrow H_{2}(\mathbb{D}) . \Gamma$ is the Hankel operator from $\ell_{2}(\mathbb{N})$ to $\ell_{2}(\mathbb{N})$.

1. Choose the dimension $N$ of the subspace to be used for the Rayleigh-Ritz method.

2. Determine the symbol $g(z)$ of the Hankel operator $\Gamma_{g}$ on $H_{2}(\mathbb{D})$ that is unitarily equivalent to $\Gamma_{G}$ using

$$
g(z)=\Pi_{+}\left(\frac{G\left(\frac{1-z}{1+z}\right)}{z}\right) .
$$

3. Determine the first $(2 N-2)$ coefficients of the Laurent series $g(z)=\sum_{k=0}^{\infty} g_{k} z^{k}$. Note that $\Gamma: \ell_{2}(\mathbb{N}) \rightarrow \ell_{2}(\mathbb{N})$ is the infinite Hankel matrix of all the coefficients with positive index of the Laurent series for $g(z) . \Gamma$ is unitarily equivalent to $\Gamma_{g}$ and to $\Gamma_{G}$.

4. Construct the truncated Hankel matrix $\hat{\Gamma}$ as

$$
\hat{\Gamma}=\left(\begin{array}{cccc}
g_{0} & g_{1} & \cdots & g_{N-1} \\
g_{1} & g_{2} & \cdots & g_{N} \\
\vdots & \vdots & \ddots & \vdots \\
g_{N-1} & g_{N} & \cdots & g_{2 N-2}
\end{array}\right) .
$$


5. Determine the eigenvalues $\left\{\mu_{k}\right\}$ of $\hat{\Gamma}$. These are approximations to the eigenvalues of $\Gamma$.

6. Determine the normalized eigenvectors $\left\{\rho_{k}\right\}$ of $\hat{\Gamma}$. These are approximations to the eigenvectors of $\Gamma$.

7. Choose an integer $k$ with $1 \leq k \leq N$. Determine $p_{k}(z)$ given by

$$
p_{k}(z)=\left(\begin{array}{llllll}
1 & z & z^{2} & z^{3} & \cdots & z^{N-1}
\end{array}\right) \rho_{k} .
$$

$p_{k}(z)$ is an approximation to the eigenvector of $\Gamma_{g}$, and $\left\|p_{k}(z)\right\|=1$ since $\rho_{k}$ is normalized. Also note that $\mu_{k}=J_{\Gamma_{g}}\left(p_{k}\right)=\left\langle\left(\Gamma_{g} p_{k}\right)(z), p_{k}(z)\right\rangle$ is an approximation to the corresponding eigenvalue of $\Gamma_{g}$.

8. Calculate $\Gamma_{g} p_{k}(z)$.

9. Calculate $e_{\Gamma_{g}}\left(p_{k}\right)=\left\|\Gamma_{g} p_{k}(z)-\mu_{k} p_{k}(z)\right\|$.

10. The $k^{\text {th }}$ non-zero eigenvalue of $\Gamma_{G}$ is in the interval $\left[\mu_{k}, \mu_{k}+e_{\Gamma_{g}}\left(p_{k}\right)\right]$ for $\mu_{k}>0$ or the interval $\left[\mu_{k}-e_{\Gamma_{g}}\left(p_{k}\right), \mu_{k}\right]$ for $\mu_{k}<0$.

11. The $k^{\text {th }}$ non-zero singular value of $\Gamma_{G}$ is in the interval $\left[\left|\mu_{k}\right|,\left|\mu_{k}\right|+e_{\Gamma_{g}}\left(p_{k}\right)\right]$.

This algorithm is an application of the Rayleigh-Ritz method discussed in Section 5.7. The estimation for the Hankel singular values is done in $\ell_{2}(\mathbb{N})$ using 
$E_{N}=\mathbb{R}^{N}$, where $\mathbb{R}^{N}$ is the set of $N$-tuples with real entries, with the standard basis

$$
\left\{\phi_{k}\right\}=\left\{\left(\begin{array}{c}
1 \\
0 \\
0 \\
\vdots \\
0
\end{array}\right),\left(\begin{array}{c}
0 \\
1 \\
0 \\
\vdots \\
0
\end{array}\right), \cdots,\left(\begin{array}{c}
0 \\
0 \\
\vdots \\
1
\end{array}\right)\right\} .
$$

Using this basis it is clear that

$$
\left\langle\hat{\Gamma} \phi_{k}, \phi_{\ell}\right\rangle=\hat{\Gamma}_{\ell k}
$$

and

$$
\left\langle\phi_{k}, \phi_{\ell}\right\rangle=\left\{\begin{array}{cc}
1, & k=\ell \\
0, & k \neq \ell
\end{array} .\right.
$$

Hence, the estimates of the Hankel singular values of $\Gamma_{g}$ are the eigenvalues of $\hat{\Gamma}$. The calculation of the overestimates is done in $H_{2}(\mathbb{D})$, using the subspace $E_{N}=$ $\operatorname{span}\left(\left\{z^{k}\right\}_{k=0}^{N-1}\right)$, which is isomorphic to $\mathbb{R}^{N}$.

7.2 Hankel Singular Values for Some Real-Order Systems

We first demonstrate this method with the second-order system presented throughout this text. We then apply the algorithm to two classes of fractional-order systems. 


\subsubsection{Second-Order Example}

Recall the second-order system introduced in Section 2.4.1, whose transfer function is

$$
G(s)=\frac{-2 s}{s^{2}+s+\frac{1}{4}},
$$

has two non-zero Hankel singular values that are both unity. We choose $N=2$ as the dimension of the subspace to be used in the Rayleigh-Ritz method. Recall from Section 6.6.1 that a symbol $g(z)$ for $\Gamma_{g}$ is

$$
\begin{aligned}
g(z) & =\frac{16(5 z-3)}{9(z-3)^{2}} \\
& =-\frac{16}{27}+\frac{16}{27} z+\frac{112}{243} z^{2}+\frac{176}{729} z^{3}+\frac{80}{729} z^{4}+\frac{304}{6561} z^{5}+\frac{368}{19683} z^{6}+\cdots
\end{aligned}
$$

Hence, $\hat{\Gamma}$ is given by

$$
\hat{\Gamma}=\left(\begin{array}{cc}
-\frac{16}{27} & \frac{16}{27} \\
\frac{16}{27} & \frac{112}{243}
\end{array}\right),
$$

which has eigenvalues

$$
\left\{\mu_{k}\right\}=\{-0.8587,0.7270\}
$$

and eigenvectors

$$
\left\{\rho_{k}\right\}=\left\{\left(\begin{array}{c}
0.9122 \\
-0.4097
\end{array}\right),\left(\begin{array}{c}
0.4097 \\
0.9122
\end{array}\right)\right\} .
$$


Subsequently, $p_{1}(z)$ and $p_{2}(z)$ are

$$
p_{1}(z)=-0.4097 z+0.9122
$$

and

$$
p_{2}(z)=0.9122 z+0.4097
$$

Note that $p_{1}(z)$ and $p_{2}(z)$ are the eigenvectors of the Hankel operator whose symbol is $-\frac{16}{27}+\frac{16}{27} z$, not those of the Hankel operator whose symbol is $g(z)$. Then $\left(\Gamma_{g} p_{1}\right)(z)$ and $\left(\Gamma_{g} p_{2}\right)(z)$ are

$$
\left(\Gamma_{g} p_{1}\right)(z)=\frac{7.866 z-7.050}{(z-3)^{2}}
$$

and

$$
\left(\Gamma_{g} p_{2}\right)(z)=\frac{4.182 z+2.6800}{(z-3)^{2}}
$$

$e_{\Gamma_{g}}\left(p_{1}\right)=0.3770$ and $e_{\Gamma_{g}}\left(p_{2}\right)=0.4648$. Thus, the Hankel singular values can be approximated by $\sigma_{1} \in[0.8587,1.236]$ and $\sigma_{2} \in[0.7270,1.192]$. This method was repeated for increasing values of $N$. Figure 7.1 shows the results.

Both the estimates and the upper bounds converge quickly to $\sigma_{1}$ and $\sigma_{2}$. In this example a sixth-order subspace yields less than $5 \%$ error in the estimate. The estimates converge more quickly than the upper bounds.

For $N>3$ the singular values $\sigma_{k}$ for $k>2$ are on the order of $10^{-16}$. Because the difference in orders of magnitude between $\sigma_{2}$ and $\sigma_{3}$ is so great, the $\sigma_{k}$ for $k \geq 3$ are considered zero. 

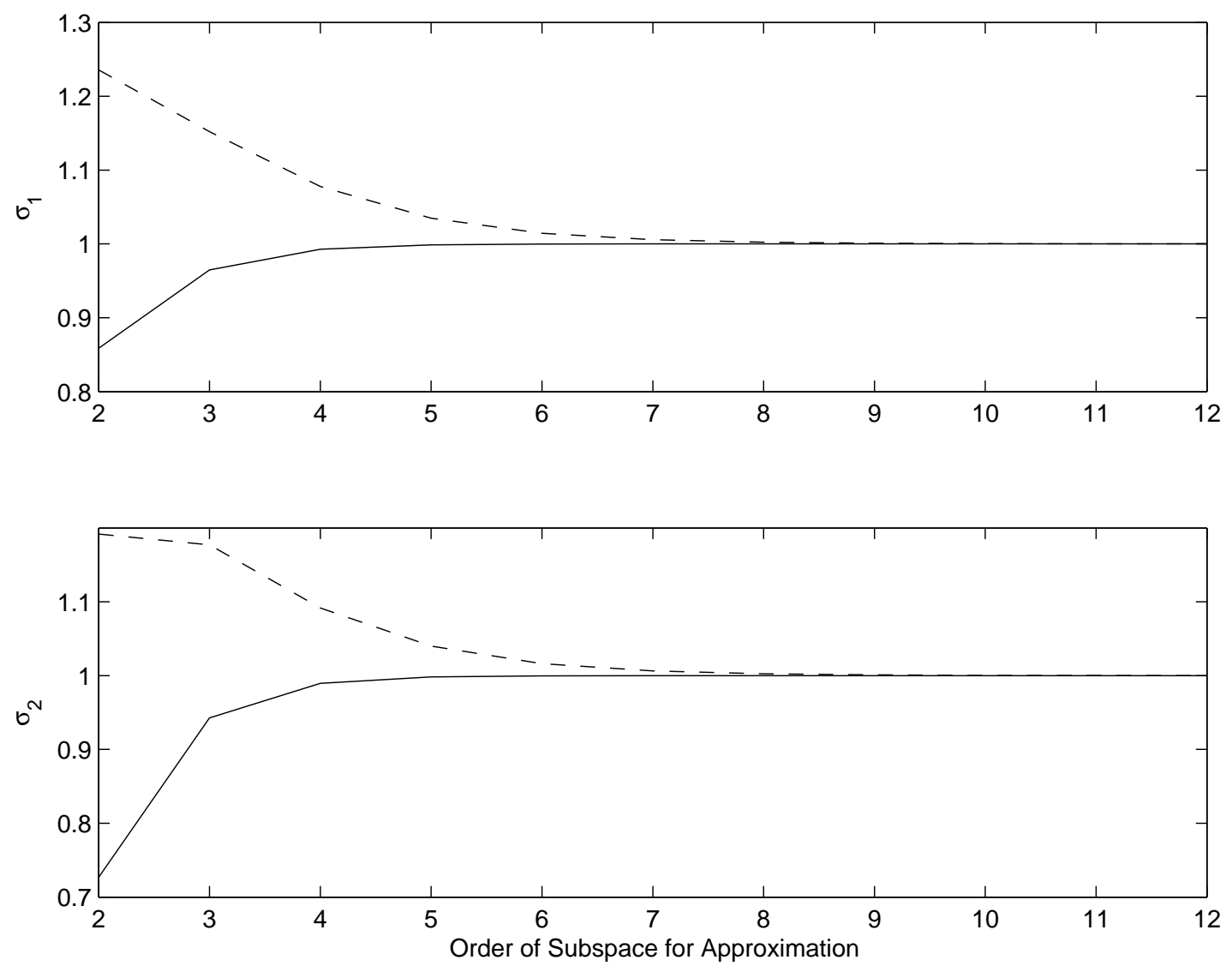

Figure 7.1: Estimates (-) and Error Bounds (- $)$ of $\sigma_{1}$ and $\sigma_{2}$ for $G(s)=\frac{-2 s}{s^{2}+s+\frac{1}{4}}$.

7.2.2 Hankel Singular Values for $\frac{a}{s^{\frac{1}{2}}+a}$

Let $G(s)$ be the system whose transfer function is

$$
G(s)=\frac{a}{s^{\frac{1}{2}}+a}
$$

By Theorem 6.5.6 and Theorem 6.5.12, $\Gamma_{G}$ is compact and Hermitian. Using the method presented in Section 7.1, estimates for the first ten Hankel singular values for the $G(s)$ were calculated for various $a \in(0,10]$. The specific values of $a$ that were 
used were

$$
\left\{\frac{1}{10}, \frac{1}{9}, \frac{1}{8}, \frac{1}{7}, \frac{1}{6}, \frac{1}{5}, \frac{1}{4}, \frac{3}{10}, \frac{1}{3}, \frac{2}{5}, \frac{1}{2}, \frac{3}{5}, \frac{7}{10}, \frac{4}{5}, \frac{9}{10}, 1,2,3,4,5,6,7,8,9,10\right\} .
$$

Table 7.1: Hankel Singular Value Bounds for $\frac{a}{s^{\frac{1}{2}}+a}$.

\begin{tabular}{cll}
\hline \hline$k$ & Lower Bound & Upper Bound \\
\hline 1 & 0.277308 & 0.278323 \\
2 & 0.102216 & 0.104107 \\
3 & 0.048703 & 0.051456 \\
4 & 0.025958 & 0.029231 \\
5 & 0.014478 & 0.017746 \\
6 & 0.008088 & 0.010877 \\
7 & 0.004413 & 0.006525 \\
8 & 0.002341 & 0.003816 \\
9 & 0.001206 & 0.002187 \\
10 & 0.000608 & 0.001244 \\
\hline \hline
\end{tabular}

The singular value estimates were equal to each other to six decimal places as $a$ varied. Similarly, the upper bounds on the singular values were equal to each other to six decimal places as $a$ varied. Table 7.1 shows these results. This leads to the following conjecture.

Conjecture 7.2.1 Fix $q \in \mathbb{R} \backslash\{0\}$. If $a \in \mathbb{R} \backslash\{0\}$, the Hankel singular values of $G(s)=\frac{a}{s^{q}+a}$ do not depend on the value of $a$. 
7.2.3 Hankel Singular Values for $\frac{1}{s^{q}+1}$ with $q \in(0,1]$

Let $G(s)$ be the system whose transfer function is given by

$$
G(s)=\frac{1}{s^{q}+1},
$$

for $q \in(0,1)$. For all such $q$, Corollary 6.5.9 gives that $\Gamma_{G}$ is a compact operator, and Theorem 6.5.12 gives that $\Gamma_{G}$ is a Hermitian operator.

Using the method presented in Section 7.1, estimates for the first ten Hankel singular values of $G(s)$ were calculated. Figure 7.2 shows the estimates of the first Hankel singular value, also called the Hankel norm, for $G(s)$ with $q \in(0,2)$, as well as an upper bound on the Hankel norm. The curves for the estimate and the upper bound can hardly be distinguished. For a closer view of the results, Figure 7.3 shows the Hankel norm of $G(s)$ for $q \in(0,1]$. Figure 7.4 through Figure 7.12 show the estimates of the second through tenth Hankel singular values of $G(s)$ with $q \in(0,1)$, as well as an upper bound on the Hankel singular value. The estimates are more easily obtained for $q \in(1,2)$ than for $q \in(0,1)$. In particular, the estimates for the third through tenth Hankel singular values are most difficult to obtain for $q$ in the neighborhood of $\frac{1}{3}$.

Recall that the system $G_{1}(s)=\frac{1}{s+1}$ is a rank 1 system whose only Hankel singular value is $\sigma_{1}=\frac{1}{2}$. Figure 7.2 demonstrates that $\lim _{q \rightarrow 1} \sigma_{1}\left(\frac{1}{s^{q}+1}\right)=\frac{1}{2}$. Figure 7.4 through Figure 7.12 demonstrate that for $2 \leq k \leq 10, \lim _{q \rightarrow 1} \sigma_{k}\left(\frac{1}{s^{q}+1}\right)=0$. These observations reinforce the results obtained. 


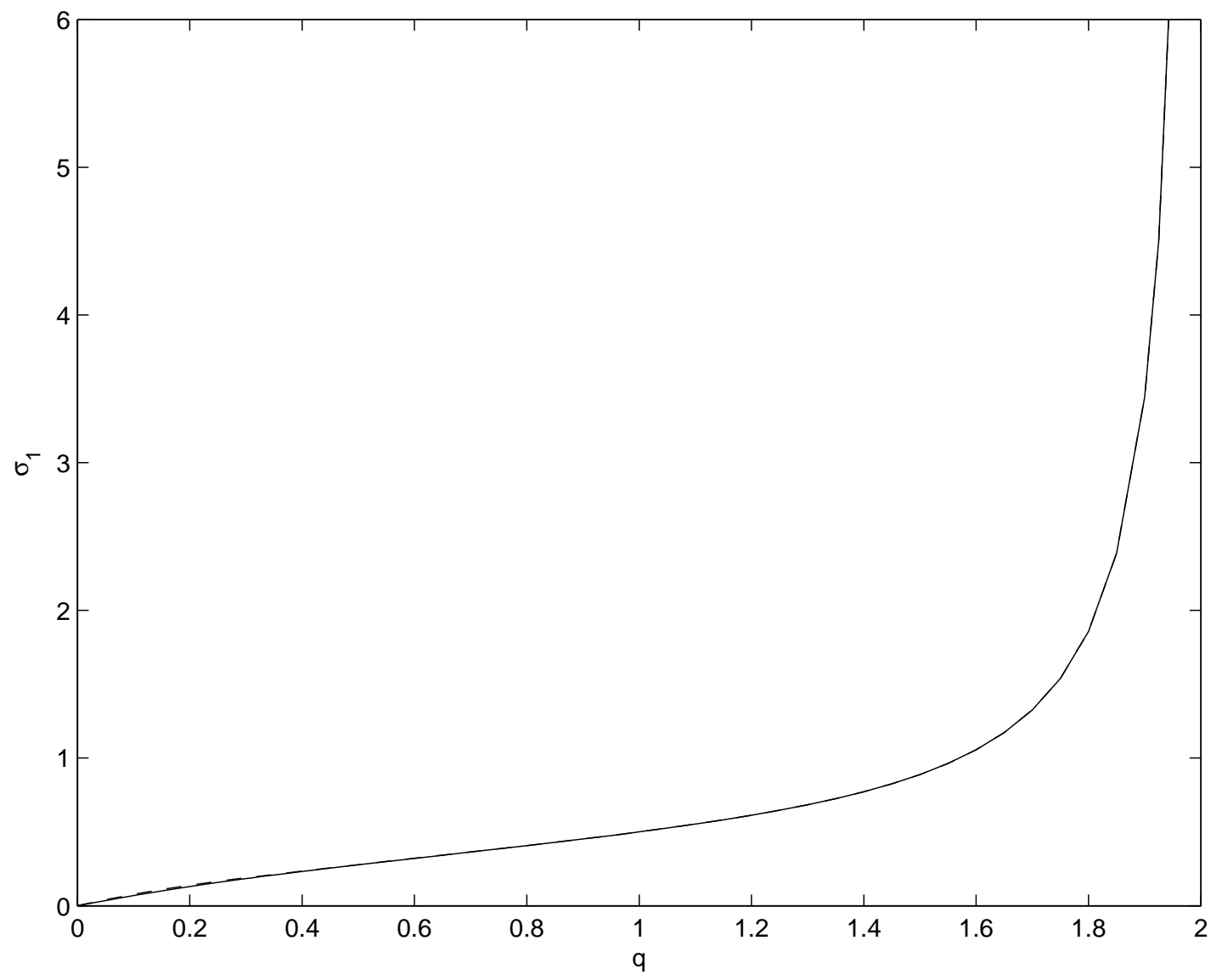

Figure 7.2: Estimates (-) and Error Bounds (- -) for $\sigma_{1}\left(\frac{1}{s^{q}+1}\right)$ for $q \in(0,2)$. 


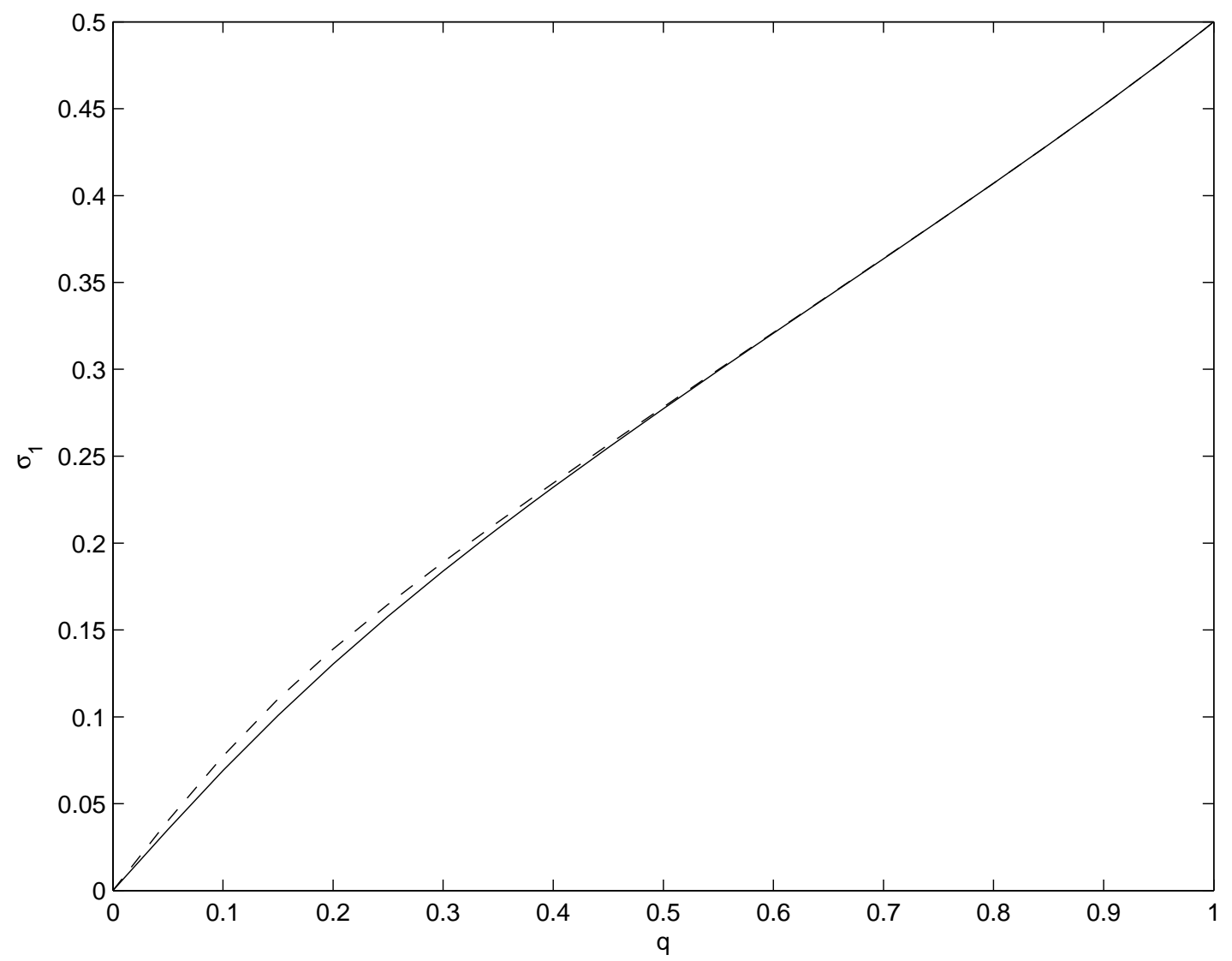

Figure 7.3: Estimates (-) and Error Bounds (- -) for $\sigma_{1}\left(\frac{1}{s^{q}+1}\right)$ for $q \in(0,1]$. 


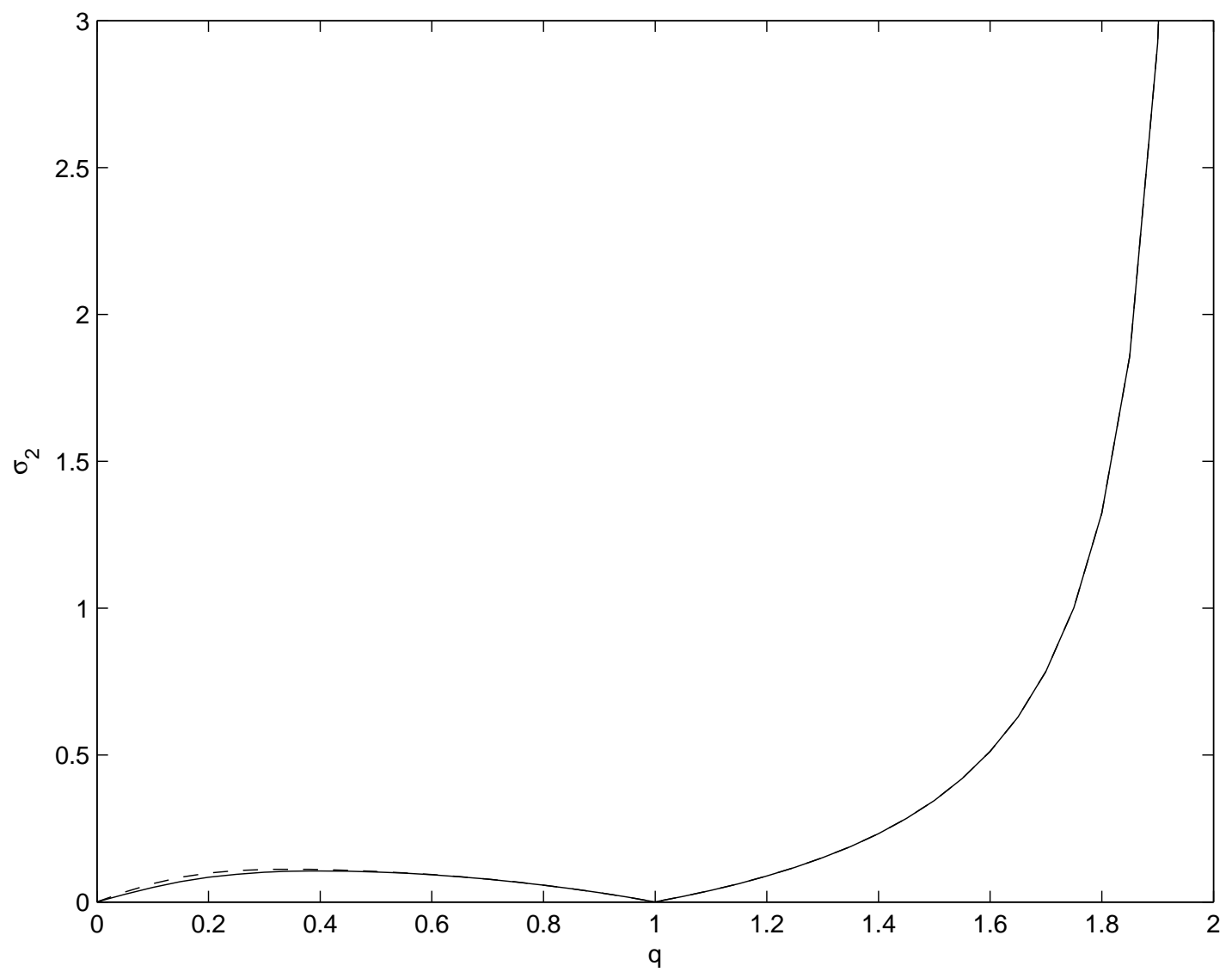

Figure 7.4: Estimates (-) and Error Bounds (- -) for $\sigma_{2}\left(\frac{1}{s^{q}+1}\right)$ for $q \in(0,2)$. 


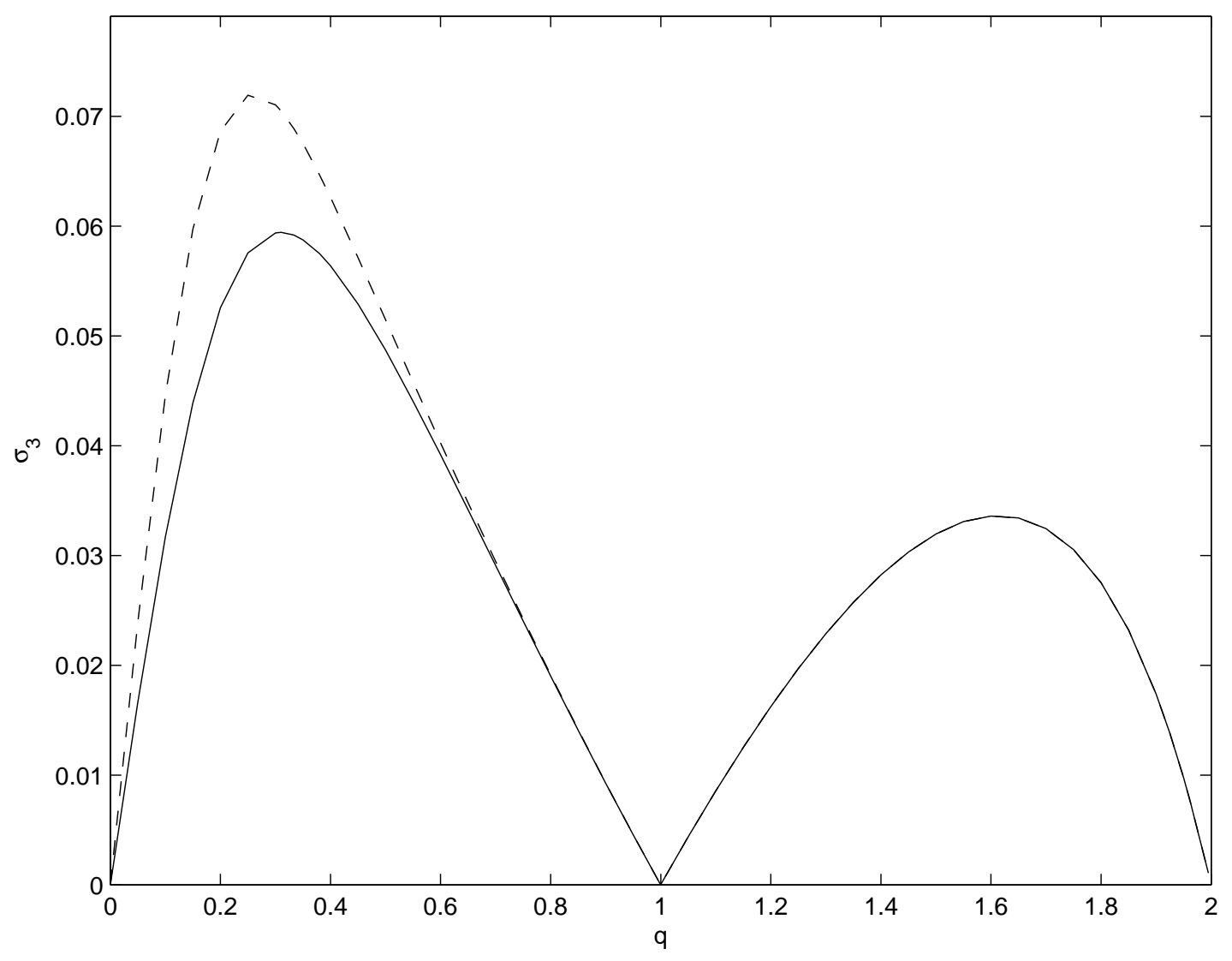

Figure 7.5: Estimates (-) and Error Bounds (- -) for $\sigma_{3}\left(\frac{1}{s^{q}+1}\right)$ for $q \in(0,2)$. 


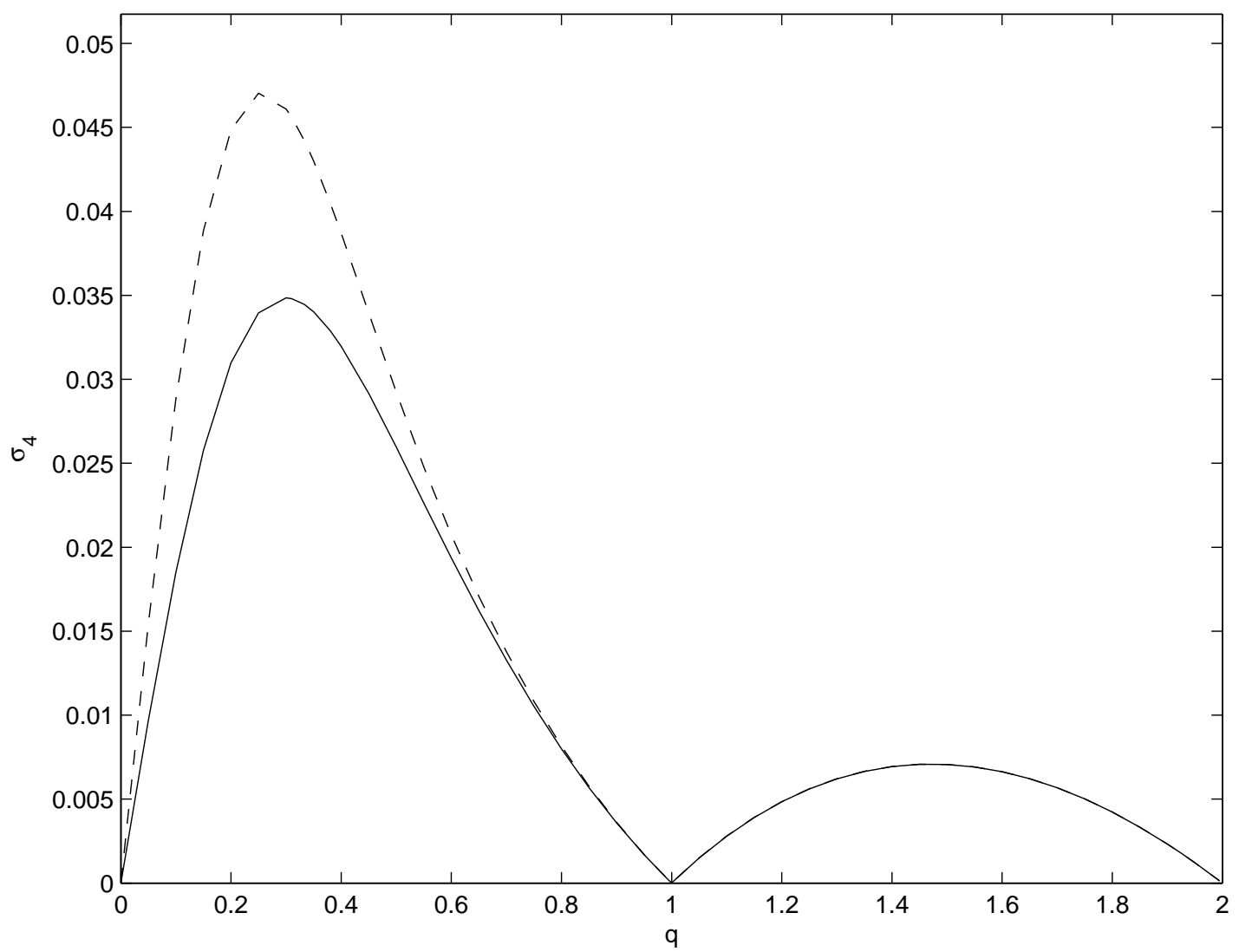

Figure 7.6: Estimates (-) and Error Bounds (- -) for $\sigma_{4}\left(\frac{1}{s^{q}+1}\right)$ for $q \in(0,2)$. 


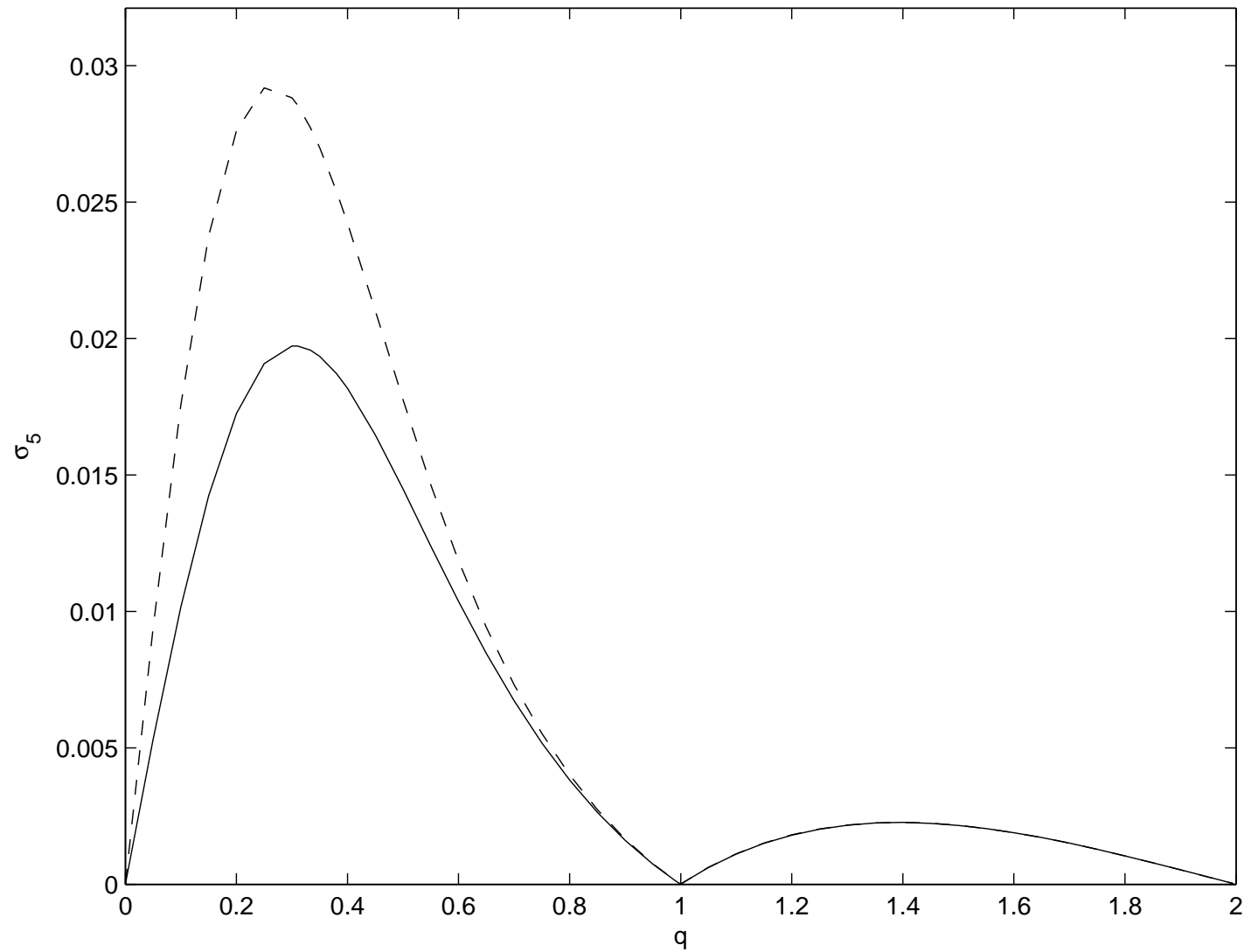

Figure 7.7: Estimates (-) and Error Bounds (- -) for $\sigma_{5}\left(\frac{1}{s^{q}+1}\right)$ for $q \in(0,2)$. 


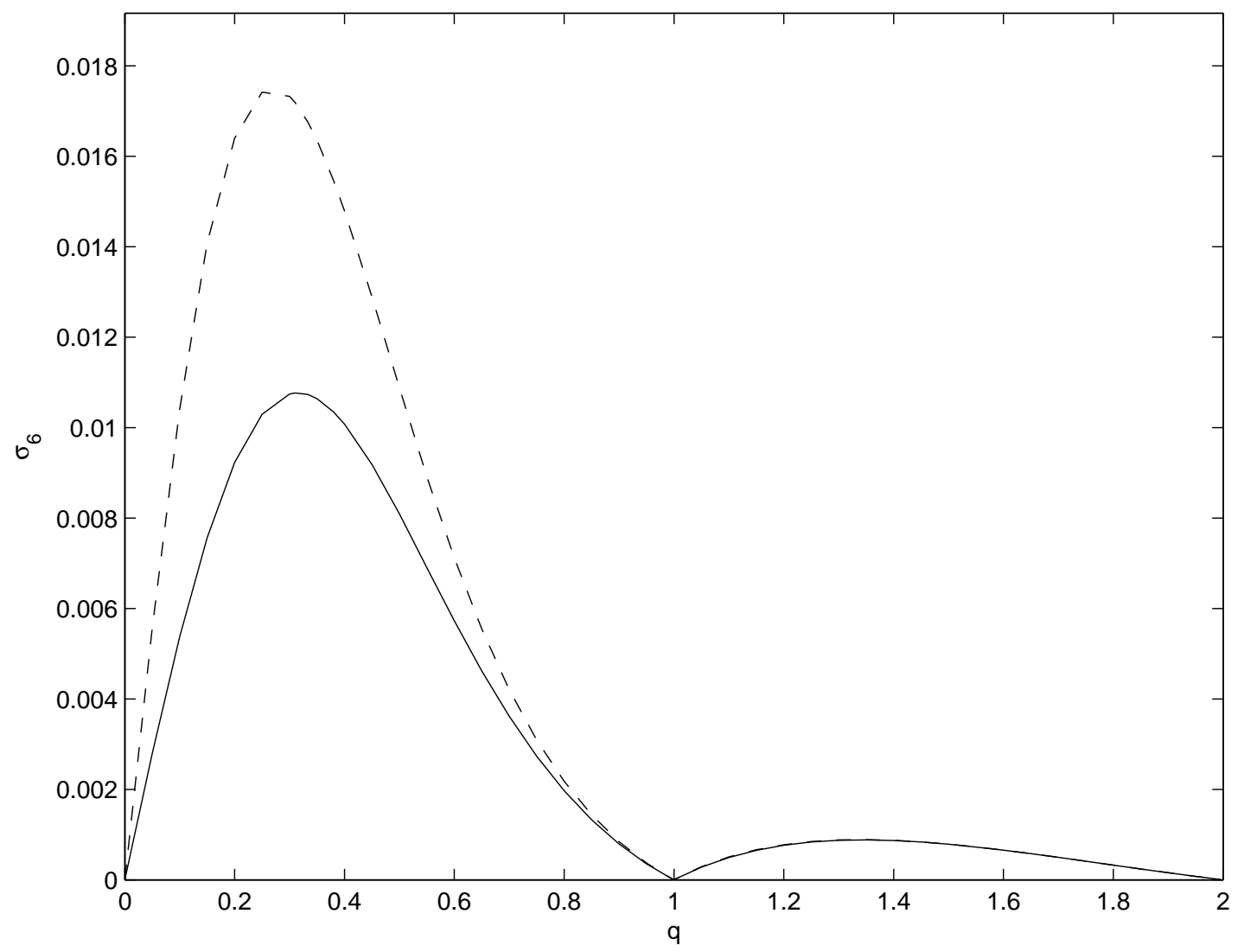

Figure 7.8: Estimates (-) and Error Bounds (- -) for $\sigma_{6}\left(\frac{1}{s^{q}+1}\right)$ for $q \in(0,2)$. 


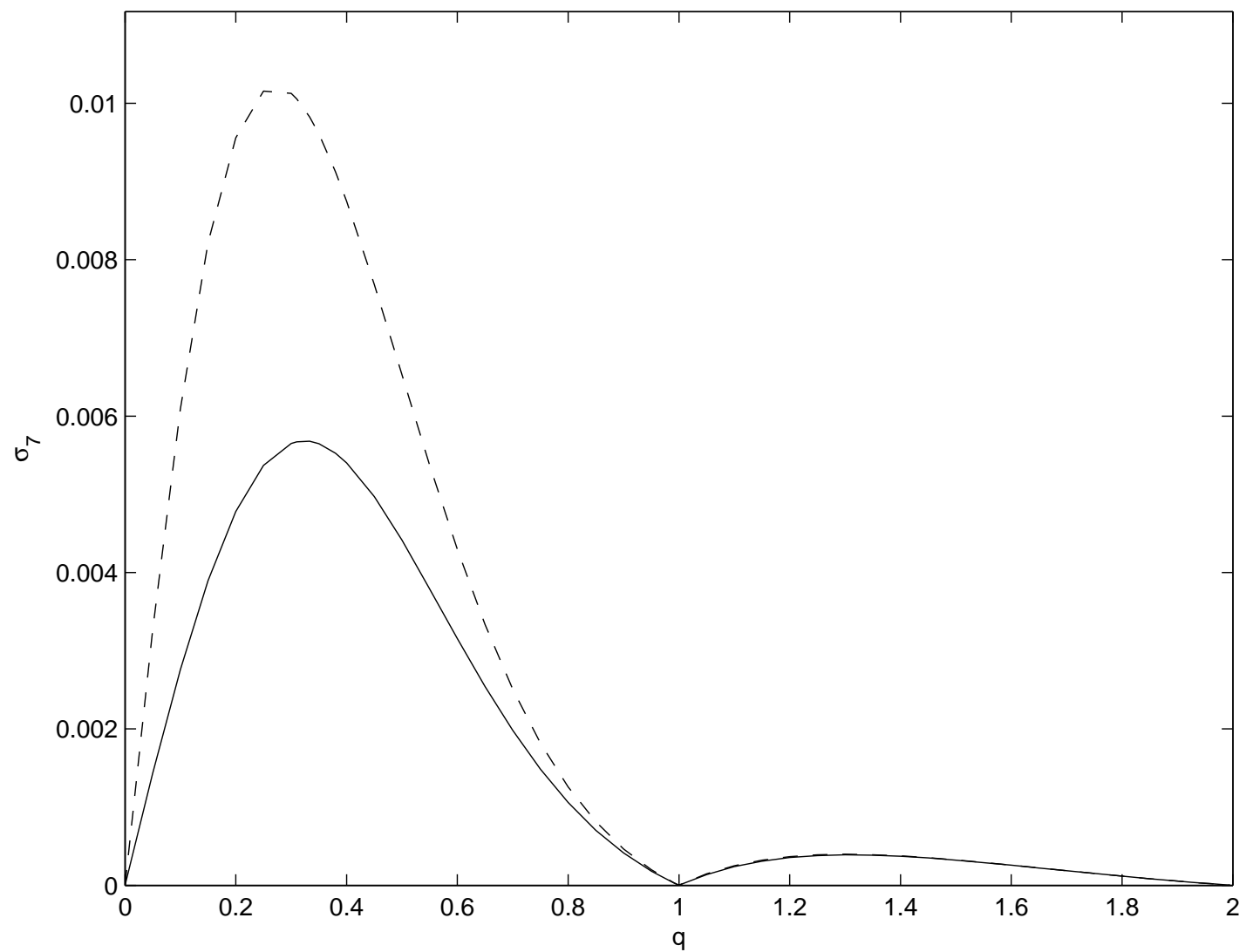

Figure 7.9: Estimates (-) and Error Bounds (- -) for $\sigma_{7}\left(\frac{1}{s^{q}+1}\right)$ for $q \in(0,2)$. 


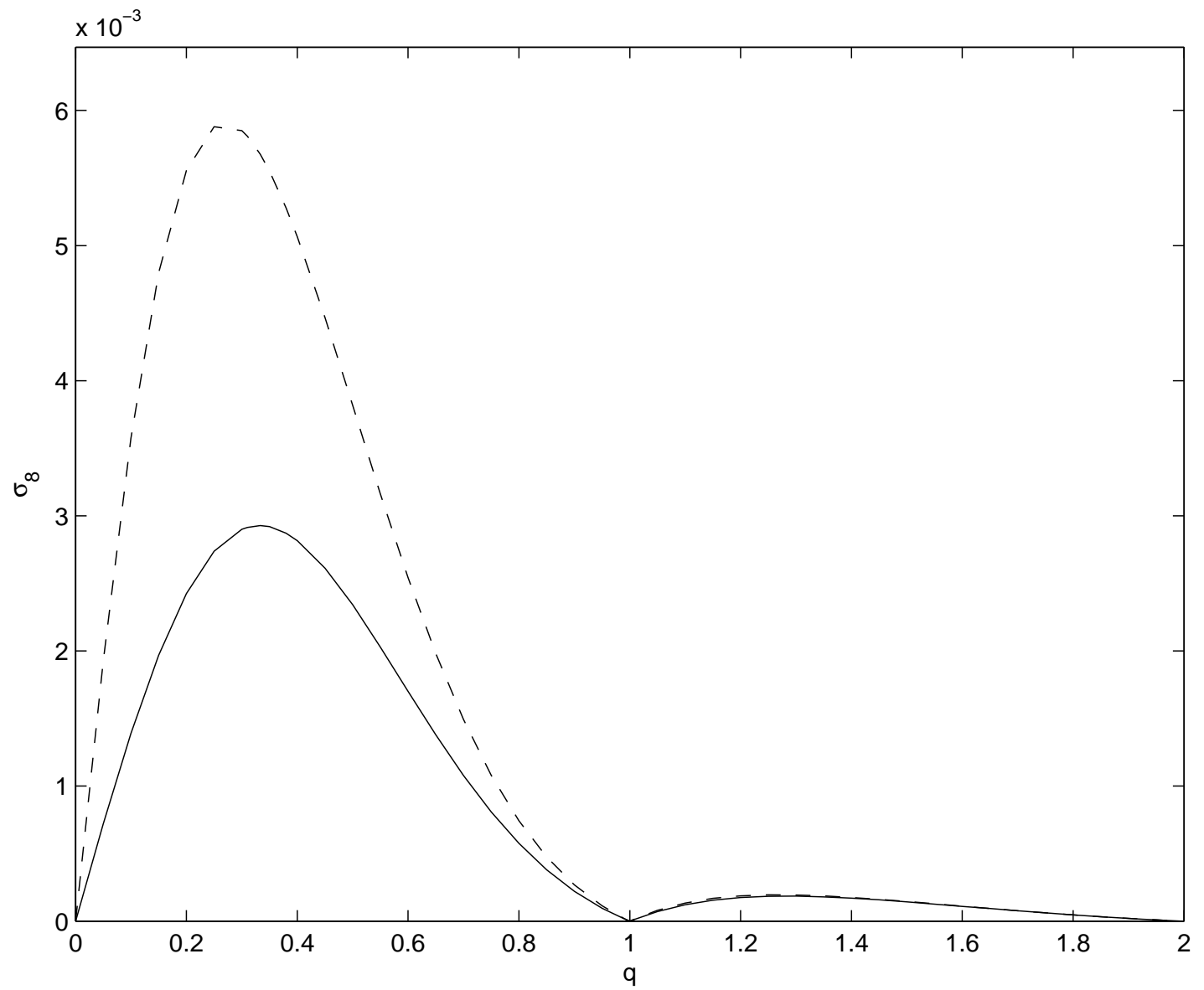

Figure 7.10: Estimates (-) and Error Bounds (- - ) for $\sigma_{8}\left(\frac{1}{s^{q}+1}\right)$ for $q \in(0,2)$. 


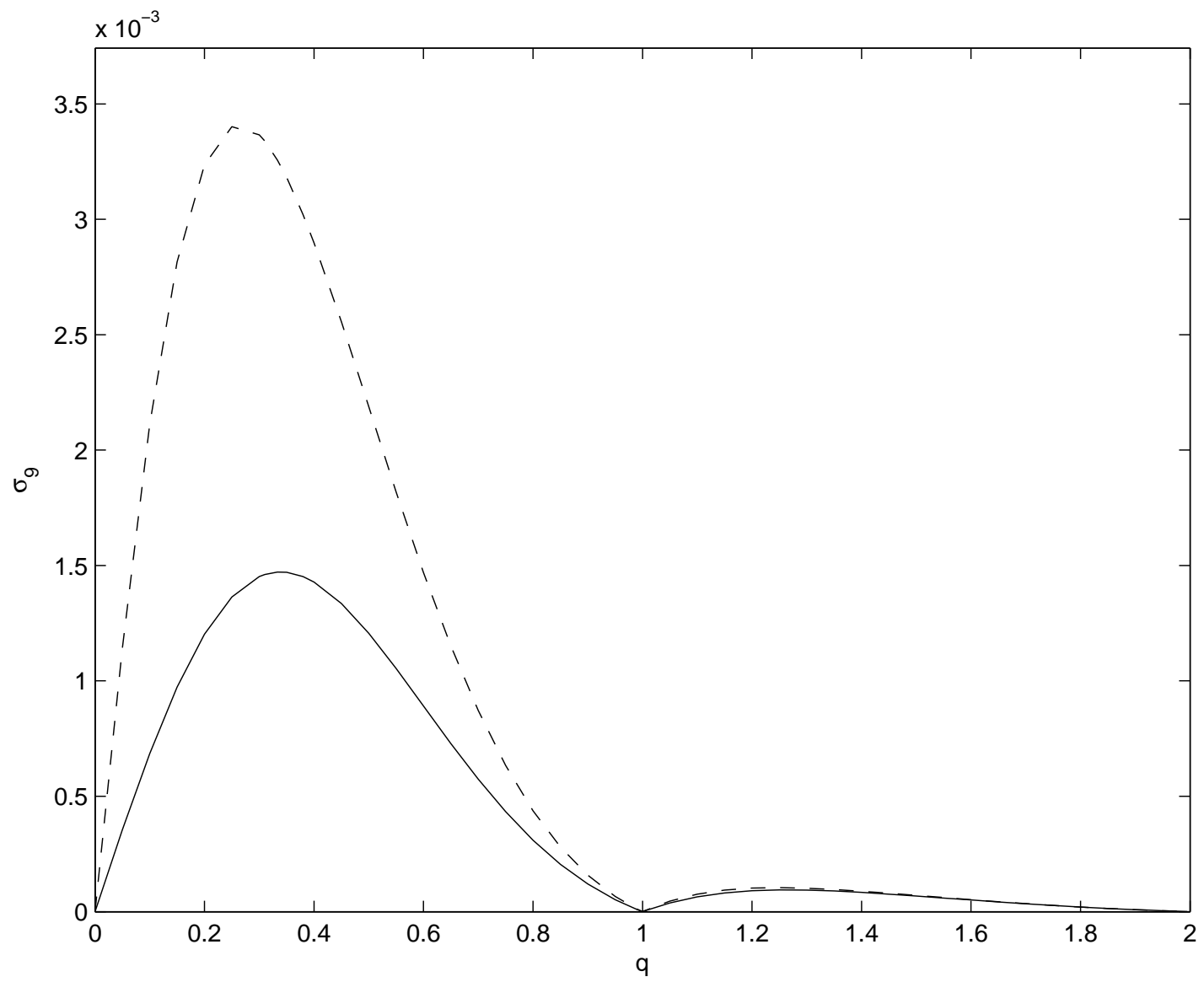

Figure 7.11: Estimates (-) and Error Bounds (- $)$ for $\sigma_{9}\left(\frac{1}{s^{q}+1}\right)$ for $q \in(0,2)$. 


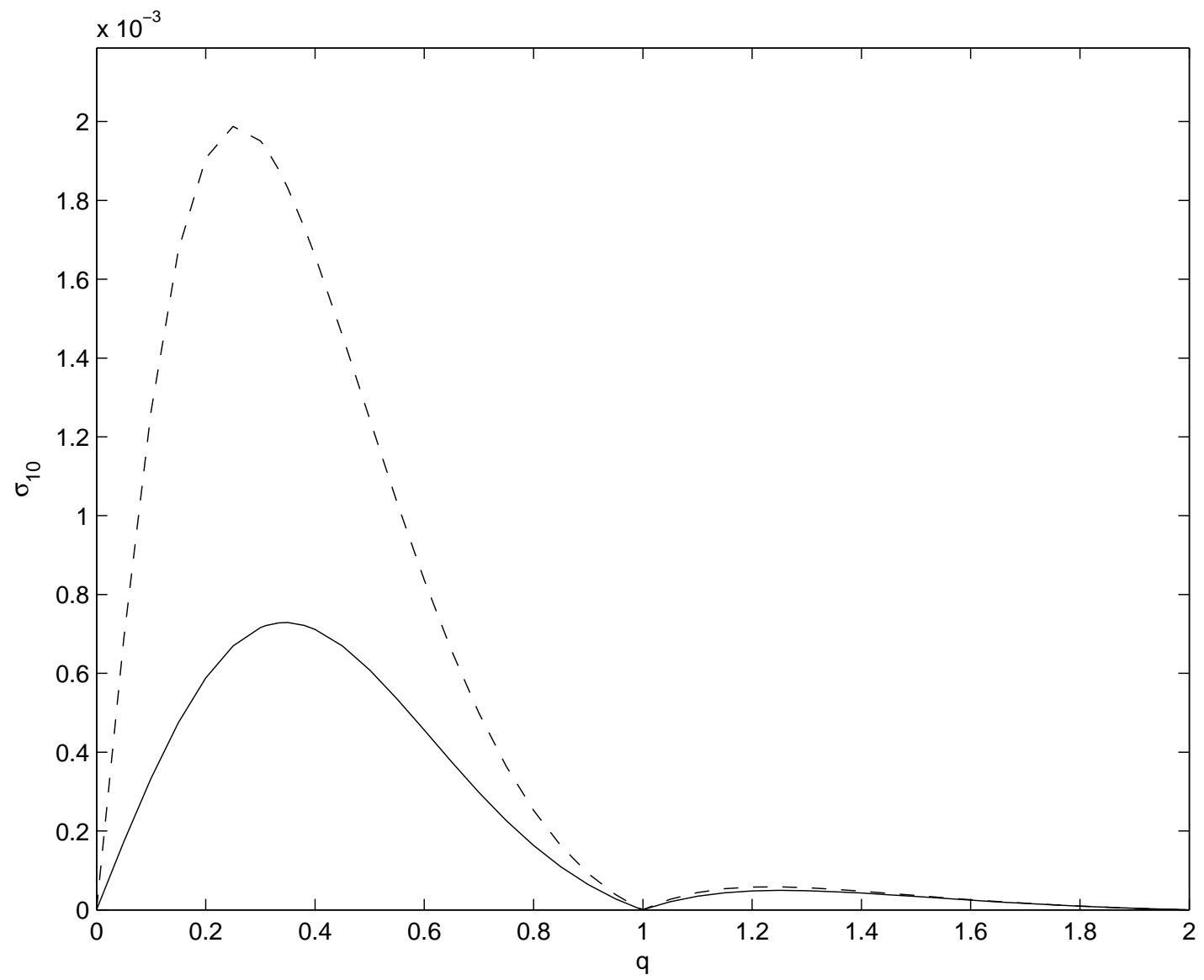

Figure 7.12: Estimates (-) and Error Bounds (- -) for $\sigma_{10}\left(\frac{1}{s^{q}+1}\right)$ for $q \in(0,2)$. 
7.2.4 An Interpretation of the Hankel Singular Values for $\frac{1}{s^{q}+1}$ with $q \in(0,1]$

$\sigma_{n+1}$ provides a lower bound on the error in Hankel norm between $\mathcal{G}$ and an $n$ dimensional approximation to $\mathcal{G}[75]$. Because this error is absolute, we may obtain lower bound on the percent error in Hankel norm between $\mathcal{G}$ and an $n$-dimensional approximation by the relation

$$
e_{n}(G(s))=\frac{\sigma_{n+1}(G(s))}{\sigma_{1}(G(s))}
$$

The estimates of $e_{n}(G(s))$, the percent errors in Hankel norm between $\mathcal{G}$ and an $n$-dimensional approximation, are given by

$$
\hat{e}_{n}(G(s))=\frac{\hat{\sigma}_{n+1}(G(s))}{\hat{\sigma}_{1}(G(s))},
$$

where $\hat{\sigma}_{k}(G(s))$ is the estimate of the $k^{\text {th }}$ Hankel singular value of $G(s)$. Upper and lower bounds on the percent error are given by

$$
\hat{e}_{u, n}(G(s))=\frac{\hat{\sigma}_{n+1}(G(s))}{\hat{\sigma}_{u, 1}(G(s))}
$$

and

$$
\hat{e}_{\ell, n}(G(s))=\frac{\hat{\sigma}_{u, n+1}(G(s))}{\hat{\sigma}_{1}(G(s))},
$$

respectively, where $\hat{\sigma}_{u, k}(G(s))$ is the upper bound on the estimate of the $k^{\text {th }}$ Hankel singular value of $G(s)$. Figures 7.13 through 7.21 show the estimates of the percent 
error in Hankel norm between $\mathcal{G}$ and a $n$-dimensional approximation as well as upper and lower bounds on the estimates. It is to be expected that the percent errors are the worst when $\sigma_{1}$ and $\sigma_{n+1}$ are near zero.

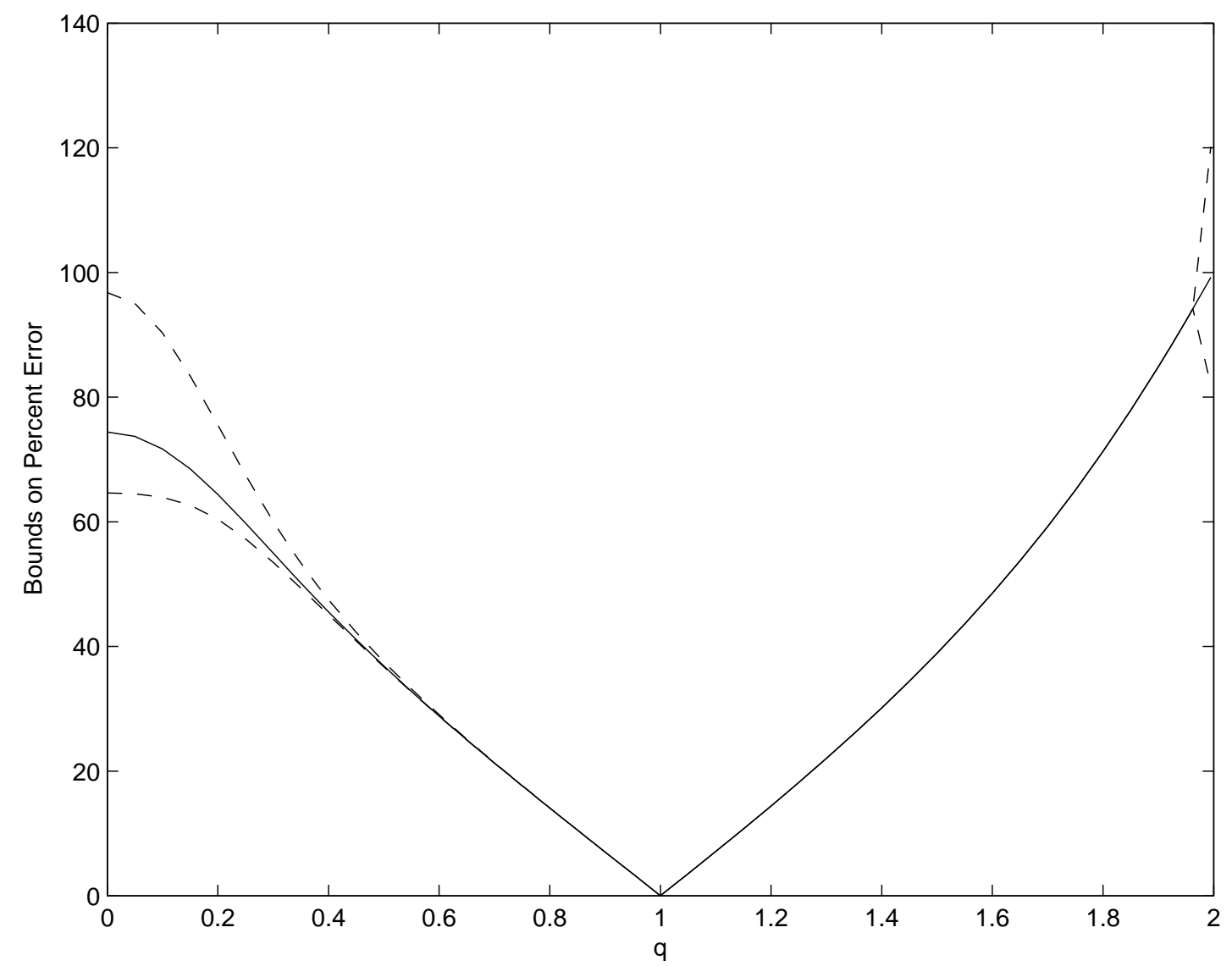

Figure 7.13: Estimates (-) and Bounds (- -) on the Percent Error in Hankel Norm Between $G(s)$ and One-Dimensional Approximation. 


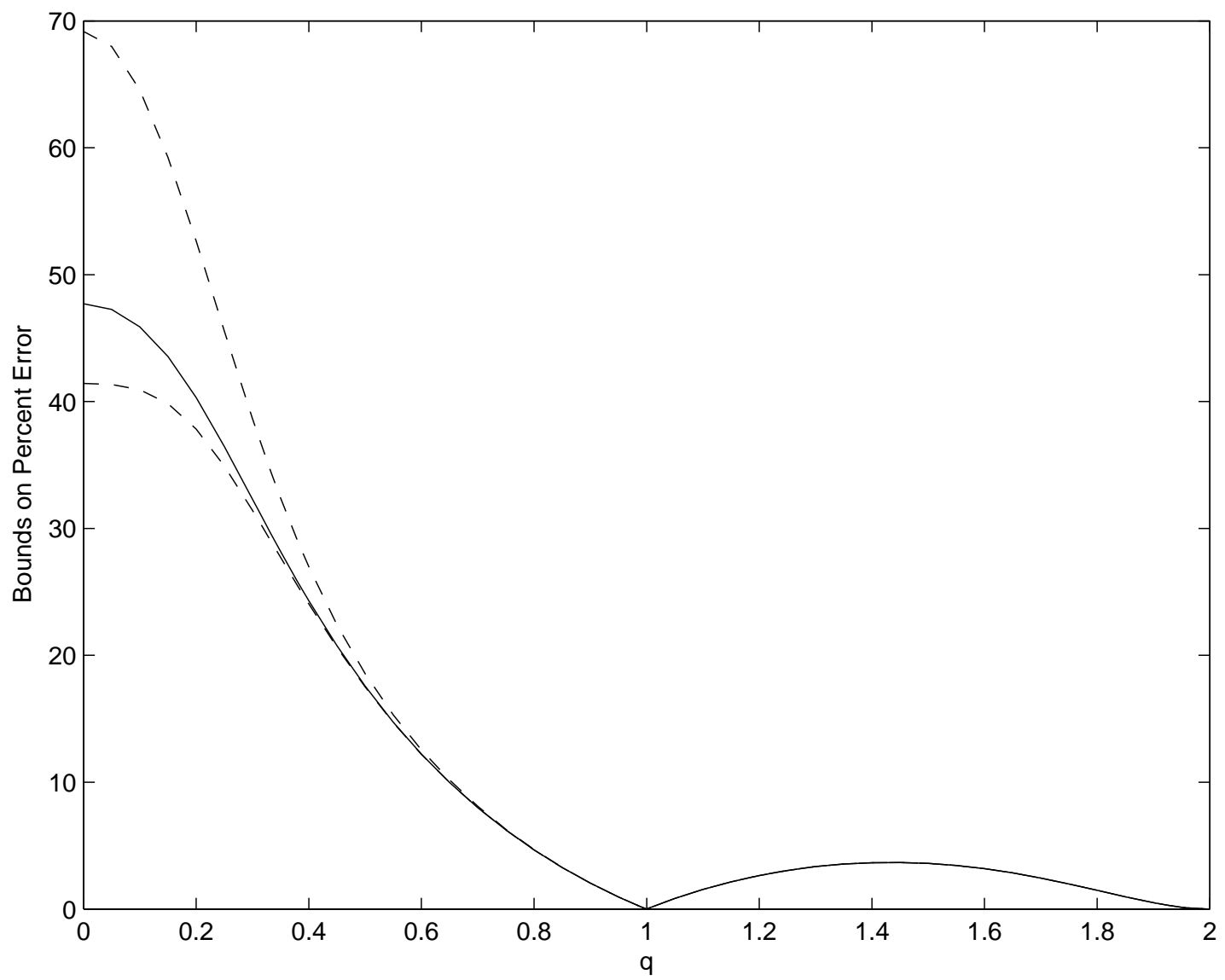

Figure 7.14: Estimates (-) and Bounds (- -) on the Percent Error in Hankel Norm Between $G(s)$ and Two-Dimensional Approximation. 


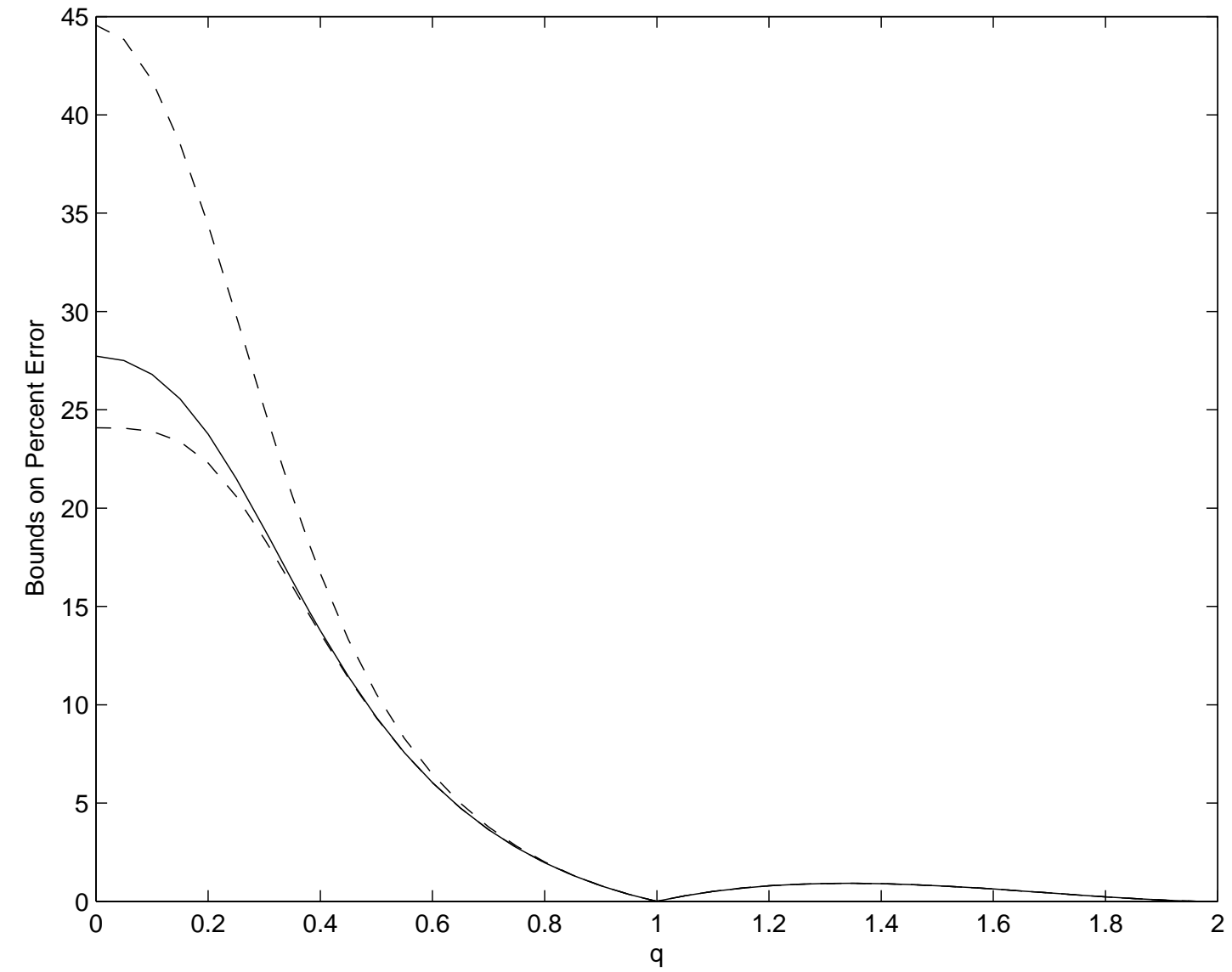

Figure 7.15: Estimates (-) and Bounds (- -) on the Percent Error in Hankel Norm Between $G(s)$ and Three-Dimensional Approximation. 


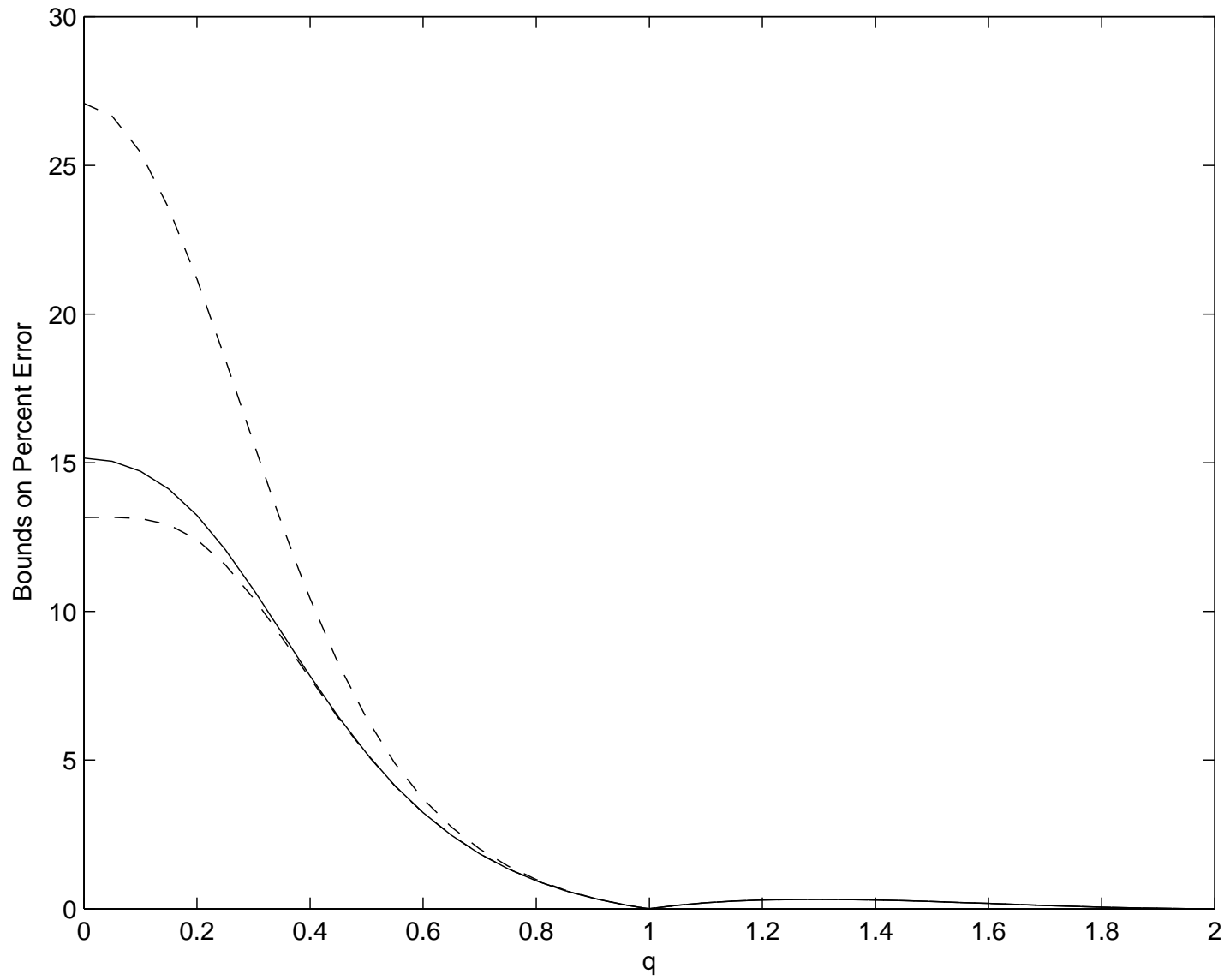

Figure 7.16: Estimates (-) and Bounds (- -) on the Percent Error in Hankel Norm Between $G(s)$ and Four-Dimensional Approximation. 


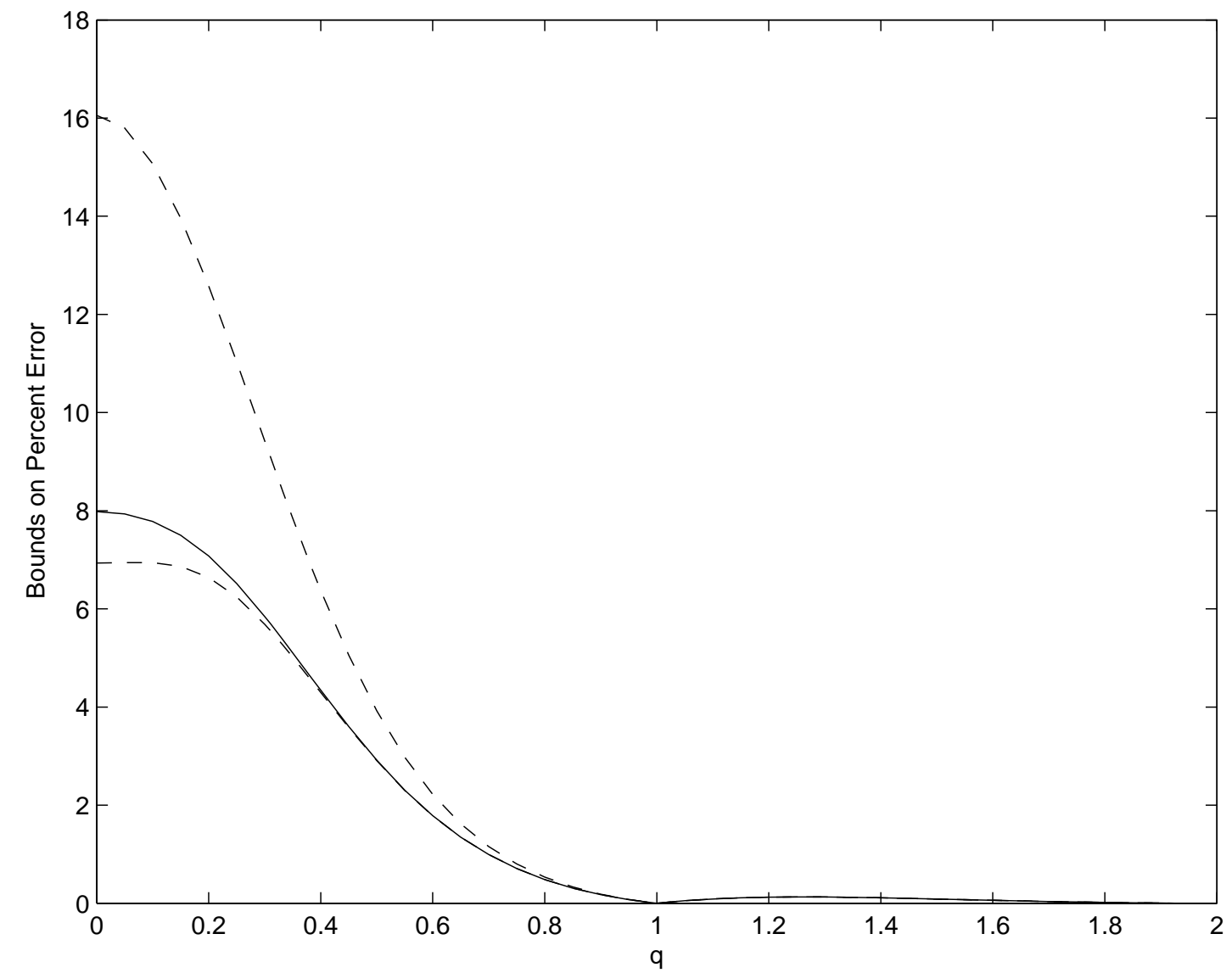

Figure 7.17: Estimates (-) and Bounds (- -) on the Percent Error in Hankel Norm Between $G(s)$ and Five-Dimensional Approximation. 


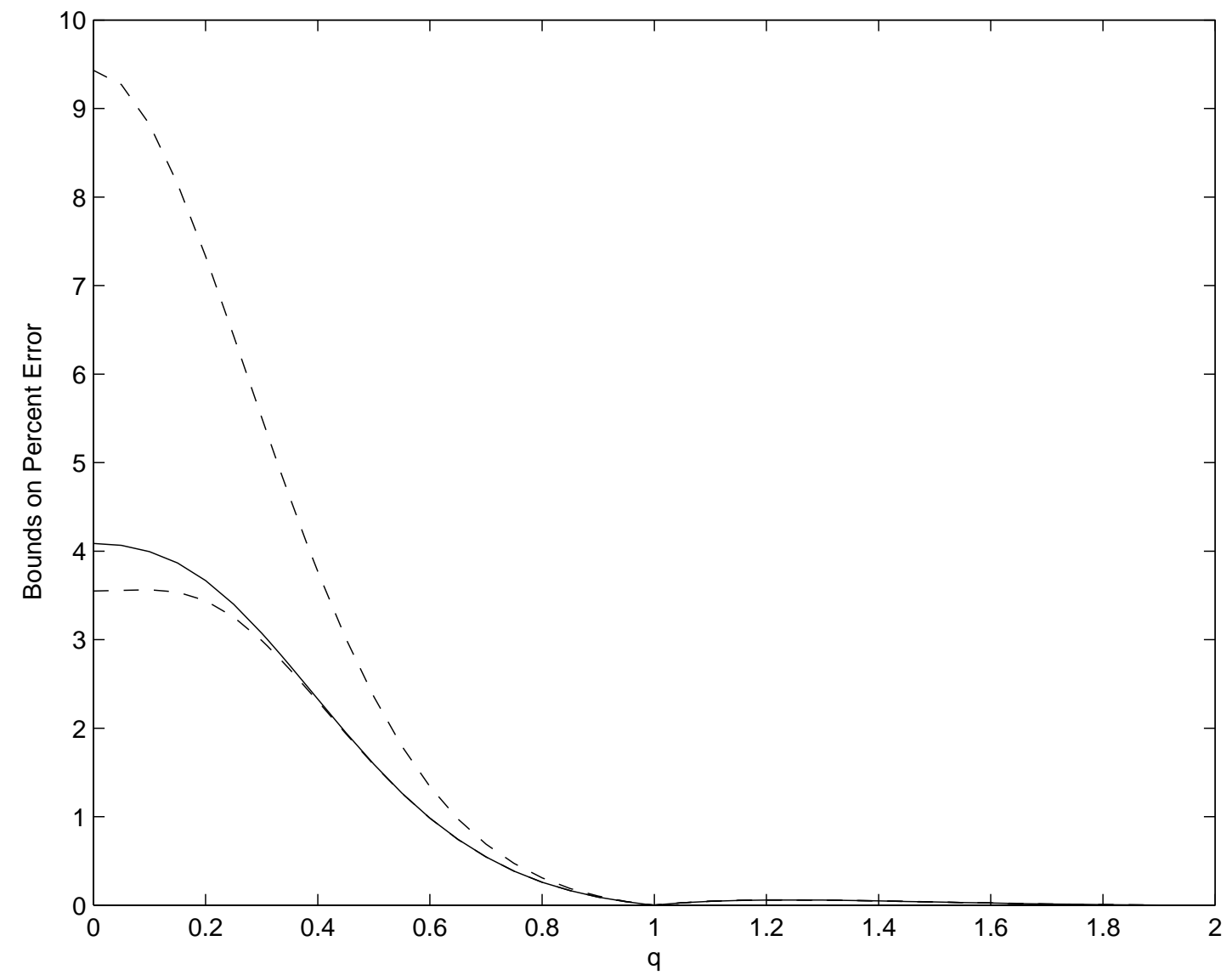

Figure 7.18: Estimates (-) and Bounds (- -) on the Percent Error in Hankel Norm Between $G(s)$ and Six-Dimensional Approximation. 


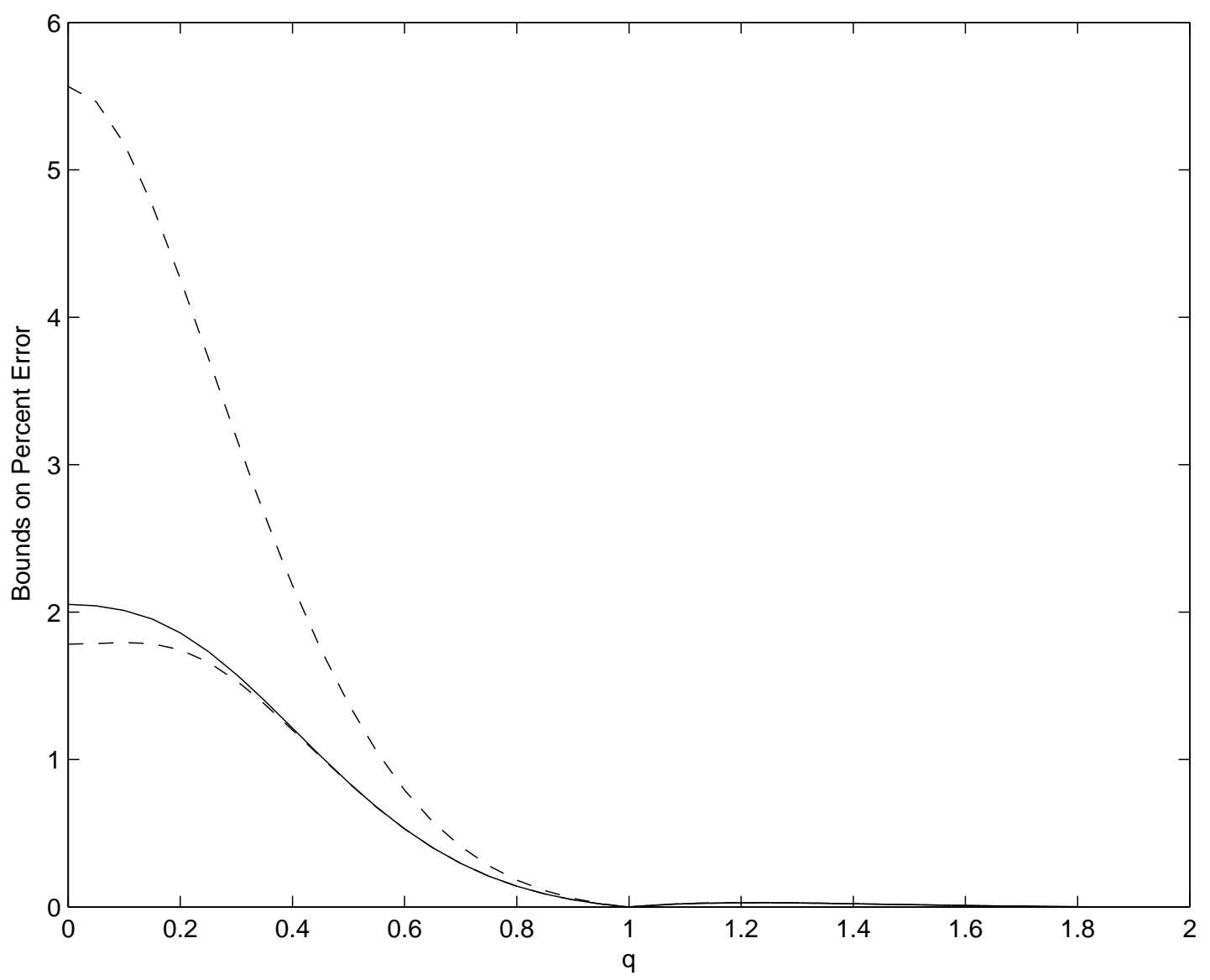

Figure 7.19: Estimates (-) and Bounds (- -) on the Percent Error in Hankel Norm Between $G(s)$ and Seven-Dimensional Approximation. 


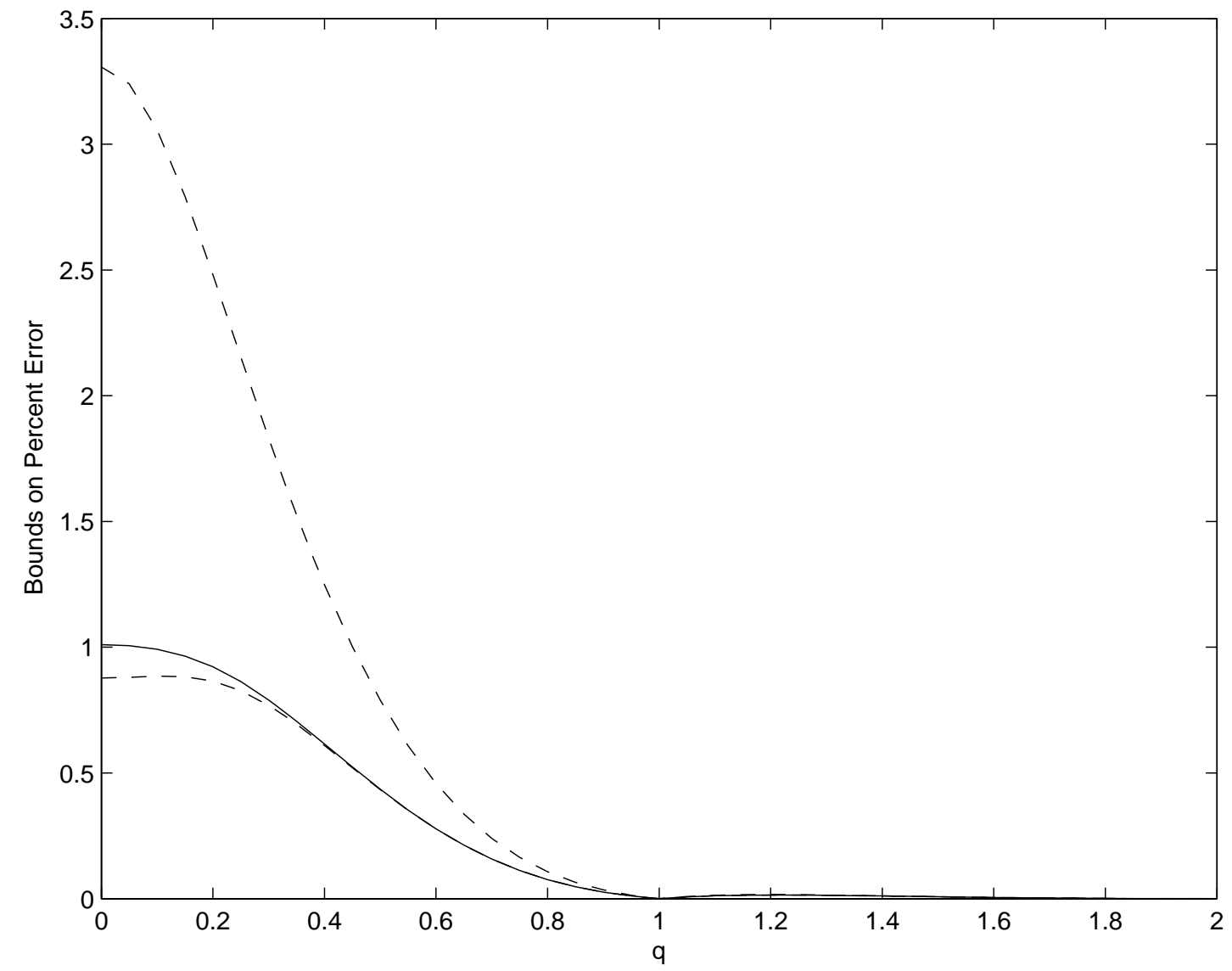

Figure 7.20: Estimates (-) and Bounds (- -) on the Percent Error in Hankel Norm Between $G(s)$ and Eight-Dimensional Approximation. 


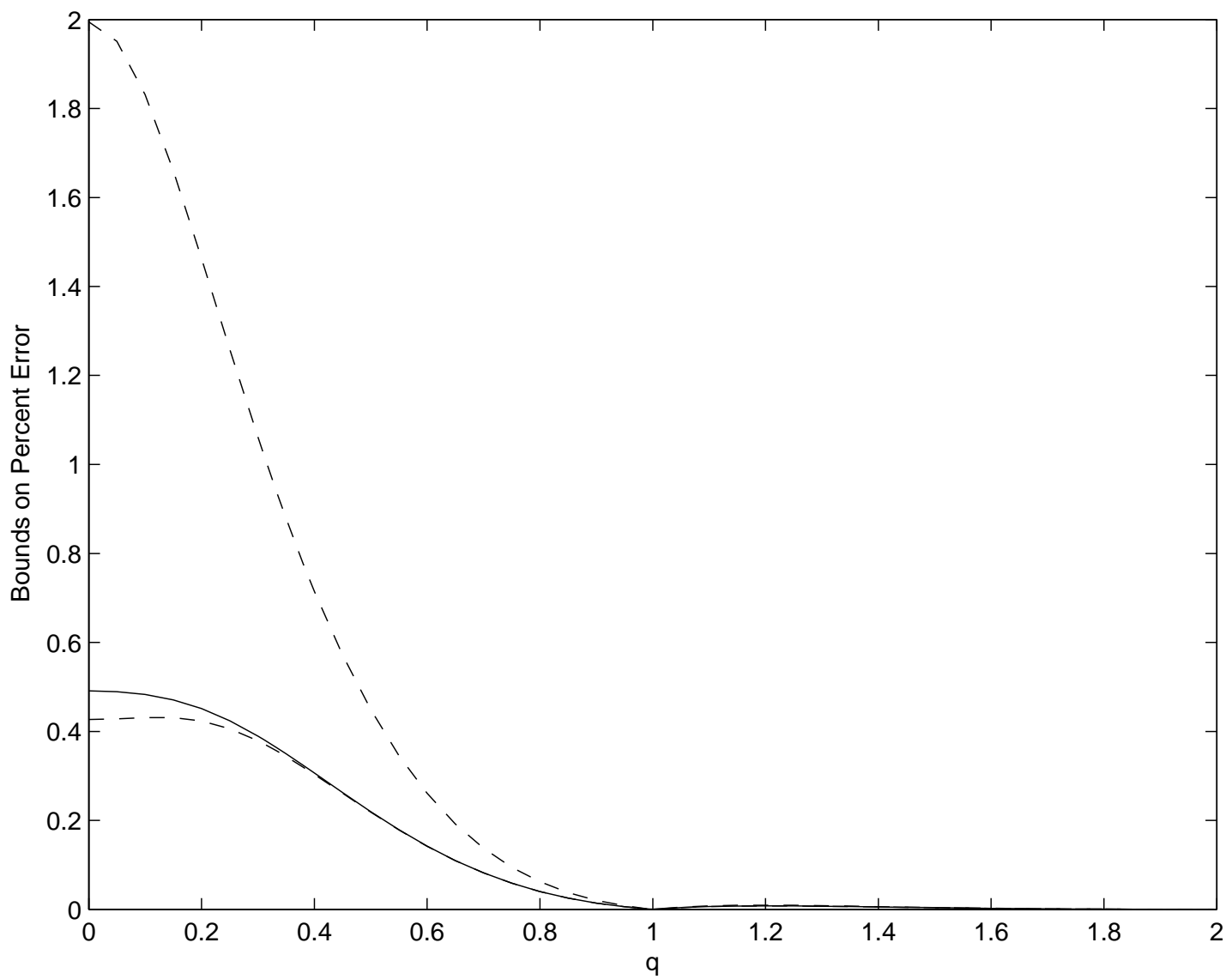

Figure 7.21: Estimates (-) and Bounds (- -) on the Percent Error in Hankel Norm Between $G(s)$ and Nine-Dimensional Approximation. 


\subsection{Examples}

We now apply the algorithm presented in Section 7.1 to the resistor-terminated and inductor-terminated semi-infinite lines.

\subsubsection{Resistor-Terminated Semi-Infinite Line}

The Hankel-singular-value approximation technique presented in Section 7.1 was applied to the resistor-terminated semi-infinite line whose transfer function is

$$
G_{R}(s)=\frac{1}{s^{\frac{1}{2}}+1} .
$$

This is a particular case of the system that was considered in Section 7.2.2, where the first ten Hankel singular values of $G_{R}(s)$ were estimated using $E_{400}$ a 400-dimensional subspace of $L_{2}(\mathbb{T})$. Because we desire to find the Hankel singular values of this system, we will use $E_{800}$ an 800-dimensional subspace, which is an extension of $E_{400}$, in our approximation. Table 7.2 shows the Hankel-singular-value estimates using $E_{800}$, the upper bound using $E_{400}$, and the upper bound using $E_{800}$. The better of the upper bounds is given in bold type.

For $M>N$ and $E_{N} \subset E_{M}, E_{M}$ can provide no worse an estimate on the Hankel singular values of a system. However, the upper bounds provided using $E_{M}$ are not necessarily better. In fact, for $6 \leq k \leq 10$, the upper bound provided by $E_{400}$ proved to be a better bound. $E_{800}$ did provide a better upper bound for $1 \leq k \leq 5$. 
Table 7.2: Hankel Singular Value Estimates $\hat{\sigma}_{k}$ and Upper Bounds for ResistorTerminated Semi-Infinite Line Using an 800-Dimensional Subspace.

\begin{tabular}{clll}
\hline \hline$k$ & $\begin{array}{l}\text { Hankel Singular } \\
\text { Value Estimate } \hat{\sigma}_{k}\end{array}$ & $\begin{array}{l}\text { Upper Bound } \\
\text { Using } E_{400}\end{array}$ & $\begin{array}{l}\text { Upper Bound } \\
\text { Using } E_{800}\end{array}$ \\
\hline 1 & 0.277311 & 0.278323 & $\mathbf{0 . 2 7 7 8 3 4}$ \\
2 & 0.102242 & 0.104107 & $\mathbf{0 . 1 0 3 2 6 2}$ \\
3 & 0.048819 & 0.051456 & $\mathbf{0 . 0 5 0 4 0 0}$ \\
4 & 0.026269 & 0.029231 & $\mathbf{0 . 0 2 8 3 1 8}$ \\
5 & 0.015035 & 0.017746 & $\mathbf{0 . 0 1 7 3 0 3}$ \\
6 & 0.008799 & $\mathbf{0 . 0 1 0 8 7 7}$ & 0.010960 \\
7 & 0.005119 & $\mathbf{0 . 0 0 6 5 2 5}$ & 0.006933 \\
8 & 0.002920 & $\mathbf{0 . 0 0 3 8 1 6}$ & 0.004303 \\
9 & 0.001627 & $\mathbf{0 . 0 0 2 1 8 7}$ & 0.002616 \\
10 & 0.000888 & $\mathbf{0 . 0 0 1 2 4 4}$ & 0.001569 \\
\hline \hline
\end{tabular}

We now consider the return on the computation investment on using an 800dimensional subspace instead of a 400-dimensional subspace. The increase in $\hat{\sigma}_{1}$ is a mere $9.99 \times 10^{-4 \%}$. For calculating the Hankel norm, very little is gained by increasing the dimension of the subspace. The increase in $\hat{\sigma}_{5}$ is $3.49 \%$, which is a modest gain, but probably not worth the effort. The most gain is in $\hat{\sigma}_{10}$, which is increased by $31.53 \%$. The decrease in upper bound ranges from $0.0176 \%$ for $\hat{\sigma}_{1}$ to $24.97 \%$ for $\hat{\sigma}_{5}$. The upper bound is actually worse for $\hat{\sigma}_{k}$ when $6 \leq k \leq 10$.

Roughly $90 \%$ of the computation time is taken with the calculation of the upper bounds. Increasing the dimension of the subspace from 400 to 800 tripled the time that it took to calculate the upper bound. Thus, the benefits in accuracy of an 
upper bound gained by increasing the dimension of the subspace are outweighed by the computation time.

Next, a 1,200-dimensional subspace $E_{1,200}$ is used in the approximation of the Hankel singular values for $G_{R}(s)$. The upper bounds determined by using $E_{400}$ and $E_{800}$ are kept. Table 7.3 shows these results, as well as the maximum possible error of the estimates. At worst, there is $17 \%$ error, with the most possible error in $\hat{\sigma}_{7}$. Table 7.4 shows the minimum possible percent error between the resistor-terminated semi-infinite line and a $k^{\text {th }}$-order approximation, calculated with the Hankel singular value estimates.

Table 7.3: Hankel Singular Value Estimates $\hat{\sigma}_{k}$ and Upper Bounds for ResistorTerminated Semi-Infinite Line Using a 1, 200-Dimensional Subspace.

\begin{tabular}{clll}
\hline \hline$k$ & $\begin{array}{l}\text { Hankel Singular } \\
\text { Value Estimate } \hat{\sigma}_{k}\end{array}$ & $\begin{array}{l}\text { Best Upper } \\
\text { Bound }\end{array}$ & $\begin{array}{l}\text { Maximum Possible } \\
\text { Error of Estimate }\end{array}$ \\
\hline 1 & 0.277311 & 0.277834 & $0.1882 \%$ \\
2 & 0.102247 & 0.103262 & $0.9822 \%$ \\
3 & 0.048847 & 0.050400 & $3.0815 \%$ \\
4 & 0.026353 & 0.028318 & $6.9409 \%$ \\
5 & 0.015212 & 0.017303 & $12.0865 \%$ \\
6 & 0.009065 & 0.010877 & $16.6608 \%$ \\
7 & 0.005423 & 0.006525 & $16.8962 \%$ \\
8 & 0.003202 & 0.003816 & $16.0693 \%$ \\
9 & 0.001854 & 0.002187 & $15.227 \%$ \\
10 & 0.001053 & 0.001244 & $15.3747 \%$ \\
\hline \hline
\end{tabular}


Table 7.4: Minimum Percent Error of Finite-Rank Approximations to the ResistorTerminated and Inductor-Terminated Semi-Infinite Lines.

\begin{tabular}{ccc}
\hline \hline$k$ & $\begin{array}{c}\text { Minimum Percent Error } \\
\text { of a Rank- } k \text { Approximation } \\
\text { to } G_{R}(s)\end{array}$ & $\begin{array}{c}\text { Minimum Percent Error } \\
\text { of a Rank- } k \text { Approximation }\end{array}$ \\
\hline 1 & $36.87 \%$ & $38.91 \%$ \\
2 & $17.62 \%$ & $3.599 \%$ \\
3 & $9.526 \%$ & $0.795 \%$ \\
4 & $5.542 \%$ & $0.243 \%$ \\
5 & $3.366 \%$ & $0.0886 \%$ \\
6 & $2.083 \%$ & $0.0363 \%$ \\
7 & $1.289 \%$ & $0.0161 \%$ \\
8 & $0.788 \%$ & $0.00765 \%$ \\
9 & $0.474 \%$ & $0.00381 \%$ \\
\hline \hline
\end{tabular}

Recall that $\hat{G}_{R}(s)$, a fifth-order approximation to $G_{R}(s)$, is given by

$$
\hat{G}_{R}(s)=\frac{13.39 s^{4}+470.4 s^{3}+1501 s^{2}+470.4 s+13.39}{s^{5}+124.49 s^{4}+1592.4 s^{3}+2623 s^{2}+481.5 s+14.39} .
$$

We can use the algorithm of Section 7.1 to determine the norm of $\Gamma_{G}-\Gamma_{\hat{G}}$, which is the Hankel norm of $G_{R}(s)-\hat{G}_{R}(s)$. Performing the approximation in a 400dimensional subspace gives that $\left\|\Gamma_{G}-\Gamma_{\hat{G}}\right\|$ is between 0.0238471 and 0.0282865 . The percent error between $\Gamma_{G}$ and $\Gamma_{\hat{G}}$ is between $8.60 \%$ and $10.20 \%$. The best fifth-order approximation has $3.366 \%$ error. The error for $\Gamma_{\hat{G}}$ is comparable to the $9.526 \%$ error accheivable by the optimal third-order approximation. Because the reduction in complexity from a fifth-order system to a third-order system is small, if $10.20 \%$ 
error is acceptable, then it is better to use $\hat{G}_{R}(s)$ than to calculate the optimal thirdorder approximation.

\subsubsection{Inductor-Terminated Semi-Infinite Line}

The Hankel-singular-value approximation technique presented in Section 7.1 was applied to the inductor-terminated semi-infinite line whose transfer function is

$$
G_{L}(s)=\frac{1}{s^{\frac{3}{2}}+1} .
$$

This is a particular case of the system that was considered in Section 7.2.3, where the first ten Hankel singular values of $G_{L}(s)$ were estimated using $E_{400}$ a 400-dimensional subspace of $L_{2}(\mathbb{T})$. Table 7.5 shows the Hankel singular value estimates, an upper bound on the estimates, and a maximum possible error for each estimate. The maximum possible error in the Hankel singular value estimates is less than $7.5 \%$. Because this error is so low, no larger subspaces will be used to approximate the Hankel singular values. Table 7.4 shows the minimum possible percent error between the inductor-terminated semi-infinite line and $k^{\text {th }}$-order approximation, calculated with the Hankel singular value estimates. Comparing the minimum possible errors for the inductor-terminated semi-infinite line with those of the resistor-terminated semi-infinite line, it is possible to achieve lower error with a $k_{0}^{\text {th }}$-order approximation to the inductor-terminated semi-infinite line than with a $k_{0}^{\text {th }}$-order approximation to the resistor-terminated semi-infinite line. 
Table 7.5: Hankel Singular Value Estimates $\hat{\sigma}_{k}$ and Upper Bounds for InductorTerminated Semi-Infinite Line Using a 400-Dimensional Subspace.

\begin{tabular}{clll}
\hline \hline$k$ & $\begin{array}{l}\text { Hankel Singular } \\
\text { Value Estimate } \hat{\sigma}_{k}\end{array}$ & $\begin{array}{l}\text { Upper Bound } \\
\text { Using } E_{400}\end{array}$ & $\begin{array}{l}\text { Maximum Possible } \\
\text { Error of Estimate }\end{array}$ \\
\hline 1 & 0.888087 & 0.888088 & $4.724 \times 10^{-5} \%$ \\
2 & 0.345511 & 0.345512 & $1.423 \times 10^{-4 \%}$ \\
3 & 0.031961 & 0.031962 & $3.012 \times 10^{-3 \%}$ \\
4 & 0.007063 & 0.007065 & $1.886 \times 10^{-2} \%$ \\
5 & 0.002157 & 0.002159 & $7.999 \times 10^{-2} \%$ \\
6 & 0.000786 & 0.000788 & $0.2656 \%$ \\
7 & 0.000321 & 0.000324 & $0.7490 \%$ \\
8 & 0.000143 & 0.000145 & $1.814 \%$ \\
9 & 0.000067 & 0.000070 & $3.879 \%$ \\
10 & 0.000033 & 0.000036 & $7.236 \%$ \\
\hline \hline
\end{tabular}

Recall that $\hat{G}_{L}(s)$, a sixth-order approximation to $G_{L}(s)$, is given by

$$
\hat{G}_{L}(s)=\frac{13.39 s^{4}+470.4 s^{3}+1501 s^{2}+470.4 s+13.39}{s^{6}+111.1 s^{5}+1135.39 s^{4}+1592.4 s^{3}+1612.1 s^{2}+471.4 s+13.39} .
$$

Once again we use the algorithm of Section 7.1 to determine the norm of $\Gamma_{G}-\Gamma_{\hat{G}}$, which is the Hankel norm of $G_{L}(s)-\hat{G}_{L}(s)$. Performing the approximation in a 60-dimensional subspace gives that $\left\|\Gamma_{G}-\Gamma_{\hat{G}}\right\|$ is between 0.0400563 and 0.0400598 . This degree of accuracy is sufficient, so we will not use a larger subspace. The percent error between $\Gamma_{G}$ and $\Gamma_{\hat{G}}$ is approximately $4.51 \%$. The best sixth-order approximation has $0.0363 \%$ error. The percent error for $\Gamma_{\hat{G}}$ is comparable to the optimal secondorder approximation, which may have a minimum of $3.599 \%$ error. If $4.51 \%$ error is acceptable, a second-order approximation can achieve that error while admitting a 
less complicated approximation. In this case it may be worthwhile to determine a second-order approximation that gives about $4.5 \%$ error.

\subsection{Conclusions}

In this chapter the details of the application of the Rayleigh-Ritz method to the estimation of the Hankel singular values of fractional-order systems were presented. The algorithm was validated with the second-order example and applied to simple

fractional-order systems of the form $G(s)=\frac{1}{s^{q}-a}$ for various $q \in(0,2)$. The first ten Hankel singular values were calculated. From the results, the Hankel singular values estimates are more accurate for $q \in(1,2)$ than they are for $q \in(0,1)$, using the same dimensional subspace. These Hankel singular values have been used to give approximations to the minimal percent errors achievable in approximating the fractional-order system with a system whose order is an integer between zero and ten. The Hankel singular values of the resistor-terminated semi-infinite line have been given to at most $17 \%$ error and the Hankel singular values of the inductorterminated semi-infinite line have been given to at most $8 \%$ error. We now apply this algorithm to approximate the Hankel norms of a class of conjugate-order systems. 


\section{CHAPTER VIII}

\section{ESTIMATION OF THE HANKEL NORM FOR A CLASS OF CONJUGATE-ORDER SYSTEMS}

We now consider a class of conjugate-order systems with transfer functions of the form

$G(s)=\frac{\frac{1}{2}}{s^{q}+1}+\frac{\frac{1}{2}}{s^{\bar{q}}+1}$. We demonstrate that $G(s)$ corresponds to a compact Hermitian operator. Values of $q$ are selected for which the Hankel-norm approximation is done. These values are selected to utilize the equivalent symbols of $G(s)$.

\subsection{Preliminary Considerations}

We will be using the method developed in Section 7.1 to estimate the Hankel norm of the conjugate-order system

$$
G(s)=\frac{\frac{1}{2}}{s^{q}+1}+\frac{\frac{1}{2}}{s^{\bar{q}}+1} .
$$

For $q \in \mathbb{C}^{+}$such that $(\operatorname{Re}(q)-1)^{2}+(\operatorname{Im}(q))^{2} \neq 1, G(s)$ satisfies the conditions of Corollary 6.5.7, and hence, $\Gamma_{G}$ is compact. Note that if $G(s)$ corresponds to a stable, anticausal system, $\Gamma_{G}=0$. By Theorem 6.5.12, $\Gamma_{G}$ is Hermitian.

We apply Theorem 4.4.3 to $G(s)$ to determine the set of $q$ for which $G(s)$ corresponds to a stable, causal system. The set of $q$ is shown in Figure 8.1. This set 
consists of two disjoint regions in the $(u, v)$-plane. The first region is the open disk of unity radius centered at $(1,0)$, and the second region is the open disk of unity radius centered at $(-1,0)$. Notice that for no $q \in i \mathbb{R} \backslash\{0\}$ does $G(s)$ correspond to a stable, causal system.

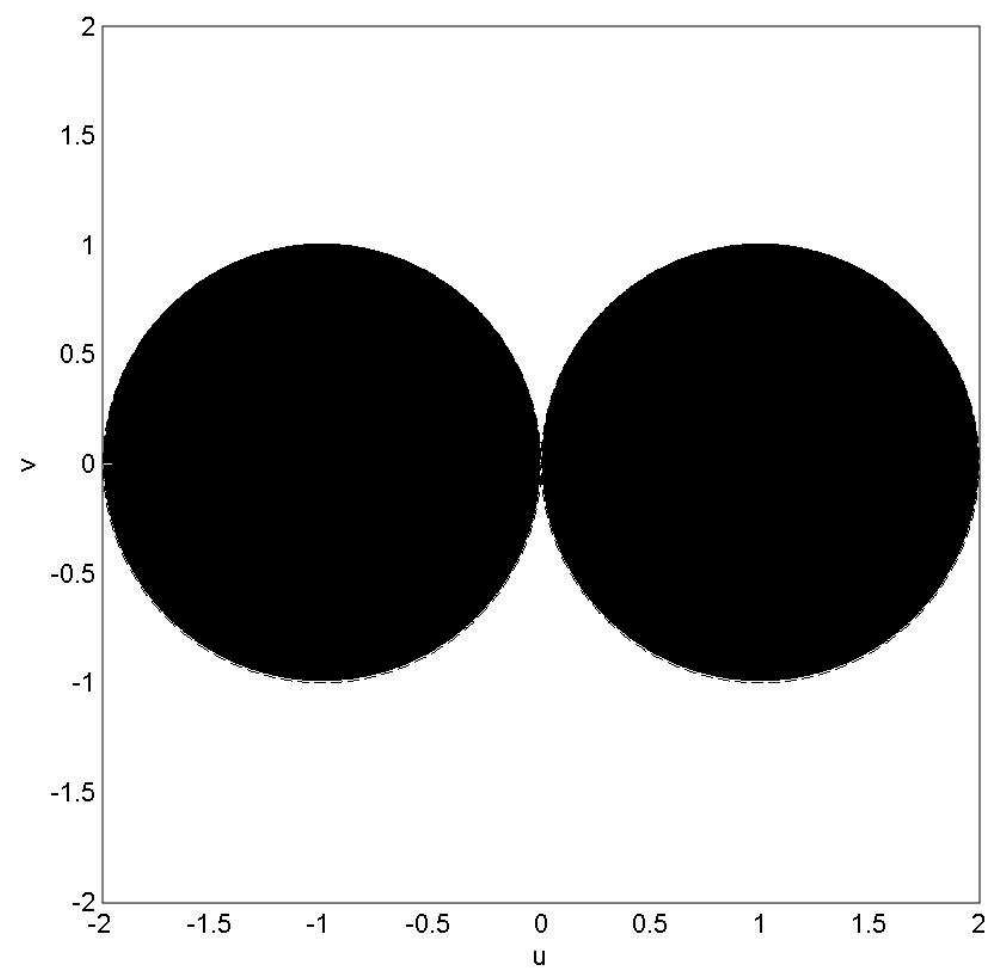

Figure 8.1: Set of $q=u+i v$ for which $G(s)=\frac{r}{s^{q}-\left(e^{i \pi}\right)}+\frac{\bar{r}}{s^{\bar{q}}-\left(e^{-i \pi}\right)}$ corresponds to a stable, causal system.

Note that

$$
G(s)=\frac{r}{s^{q}-a}+\frac{\bar{r}}{s^{\bar{q}}-\bar{a}}
$$

where $r=\bar{r}=\frac{1}{2}$ and $a=\bar{a}=-1$. Then, by Corollary 6.4.2, the Hankel operator with symbol $-\frac{\frac{1}{2}}{s^{-q}+1}-\frac{\frac{1}{2}}{s^{-\bar{q}}+1}$ is the same as the Hankel operator with symbol $\frac{\frac{1}{2}}{s^{q}+1}+\frac{\frac{1}{2}}{s^{\bar{q}}+1}$ 
because $\frac{r}{a^{2}}=\frac{\bar{r}}{\bar{a}^{2}}=\frac{1}{2}$ and $\frac{1}{a}=\frac{1}{\bar{a}}=1$. Also note that, for this system, the Hankel operator is the same for a given $q \in \mathbb{C}$ and its complex conjugate.

Because the Hankel singular values for $G(s)$ and $-G(s)$ are the same, the Hankel singular values of $G(s)=\frac{\frac{1}{2}}{s^{q}+1}+\frac{\frac{1}{2}}{s^{\bar{q}}+1}$ are equal for $q=u+i v, q=u-i v$, $q=-u+i v$ and $q=-u-i v$. Thus, we will calculate Hankel norms for systems where $q$ has positive real part and positive imaginary parts.

\subsection{Estimation}

The points shown in Figure 8.2 are the values of $q$ that will be considered. The circle $D_{0}^{+}(-1)$ centered at $q=1$ with unit radius indicates the set of $q$ for which $\Gamma_{G}$ is an unbounded operator. Thus, the points on this circle are marked with squares and are ignored. The points outside of the circle are marked with circles and are the $q$ for which $G(s)$ corresponds to a stable, anticausal system, so $\Gamma_{G}=0$. The points marked with stars are the $q$ to which the Hankel-norm approximation algorithm of Section 7.1 will be applied. We will call this set $\mathcal{P}$.

We use the method presented in Section 7.1 with an eight-hundred dimensional subspace to obtain bounded estimates of the Hankel norm of $G(s)$ for each $q \in \mathcal{P}$. Figure 8.3 and Figure 8.4 show the results of these estimations. Using symmetry, these results are extended to $q$ with negative imaginary or negative real parts. As $q$ gets closer to $D_{0}^{+}(-1),\left\|\Gamma_{G}\right\|$ increases without bound. Thus, to prevent a few values from dominating the graph and obscuring the general trend, the maximum 
value shown in Figure 8.3 is 2. Figure 8.4 shows a contour plot of this data. Note that the curve on the $\operatorname{Re}(q)$ axis is the curve shown in Figure 7.2, which is to be expected.

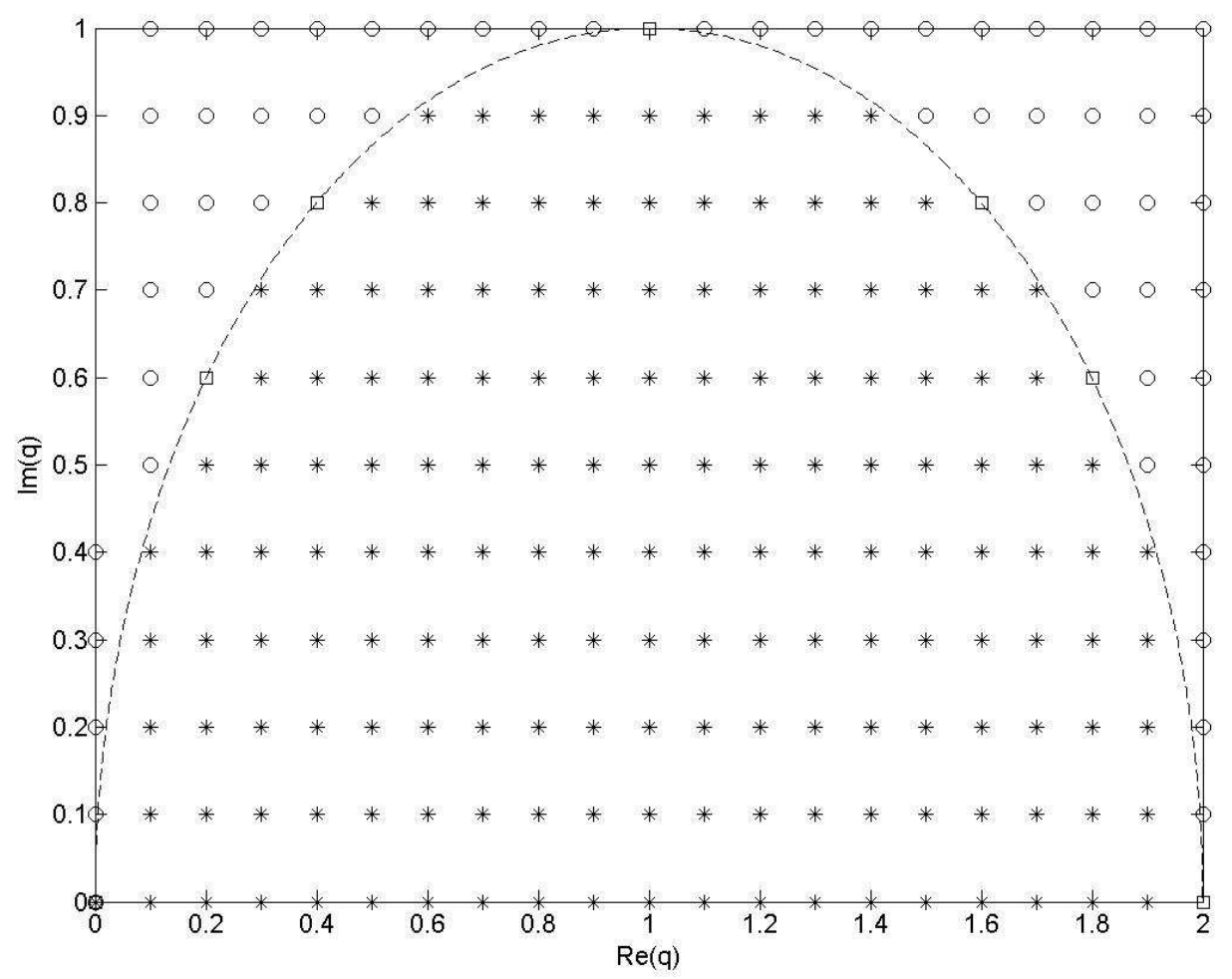

Figure 8.2: Set of $q=u+i v$ for which the Hankel norm $G(s)=\frac{r}{s^{q}-\left(e^{i \pi}\right)}+\frac{\bar{r}}{s^{\bar{q}}-\left(e^{-i \pi}\right)}$ is calculated.

The equivalence of the Hankel operator for $G(s)$ with $q$ and $\bar{q}$ is used to obtain the Hankel-norm approximations for $\operatorname{Im}(q)<0$. Figure 8.5 shows these values, and Figure 8.6 shows a contour plot of these values. Figure 8.7 shows a contour plot of the Hankel norms estimated for $q \in \mathbb{C}$. 


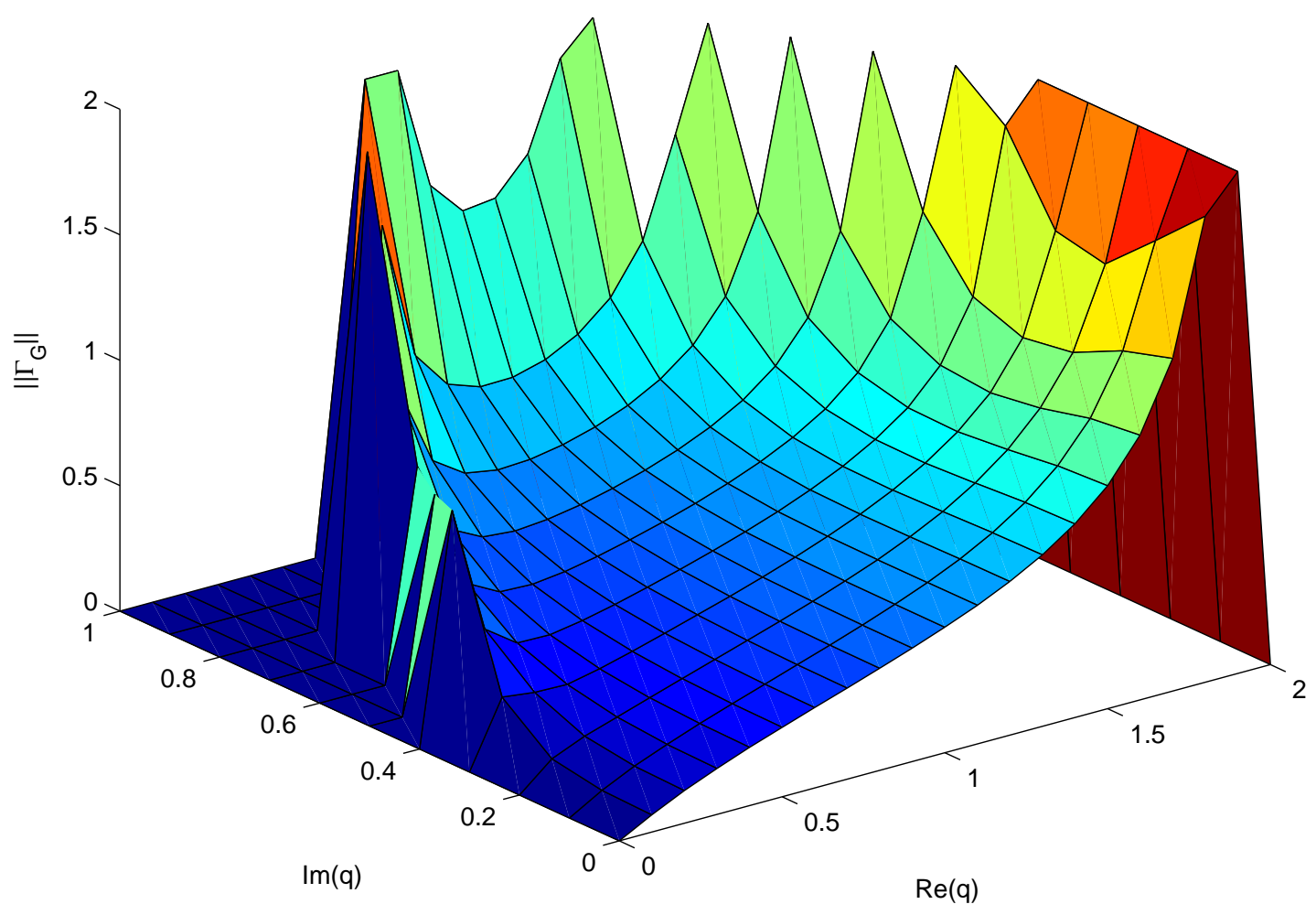

Figure 8.3: Estimates of the Hankel norm of $G(s)$ with $q \in \mathbb{C}$ such that $0 \leq \operatorname{Re}(q) \leq 2$ and $0 \leq \operatorname{Im}(q) \leq 1$. 


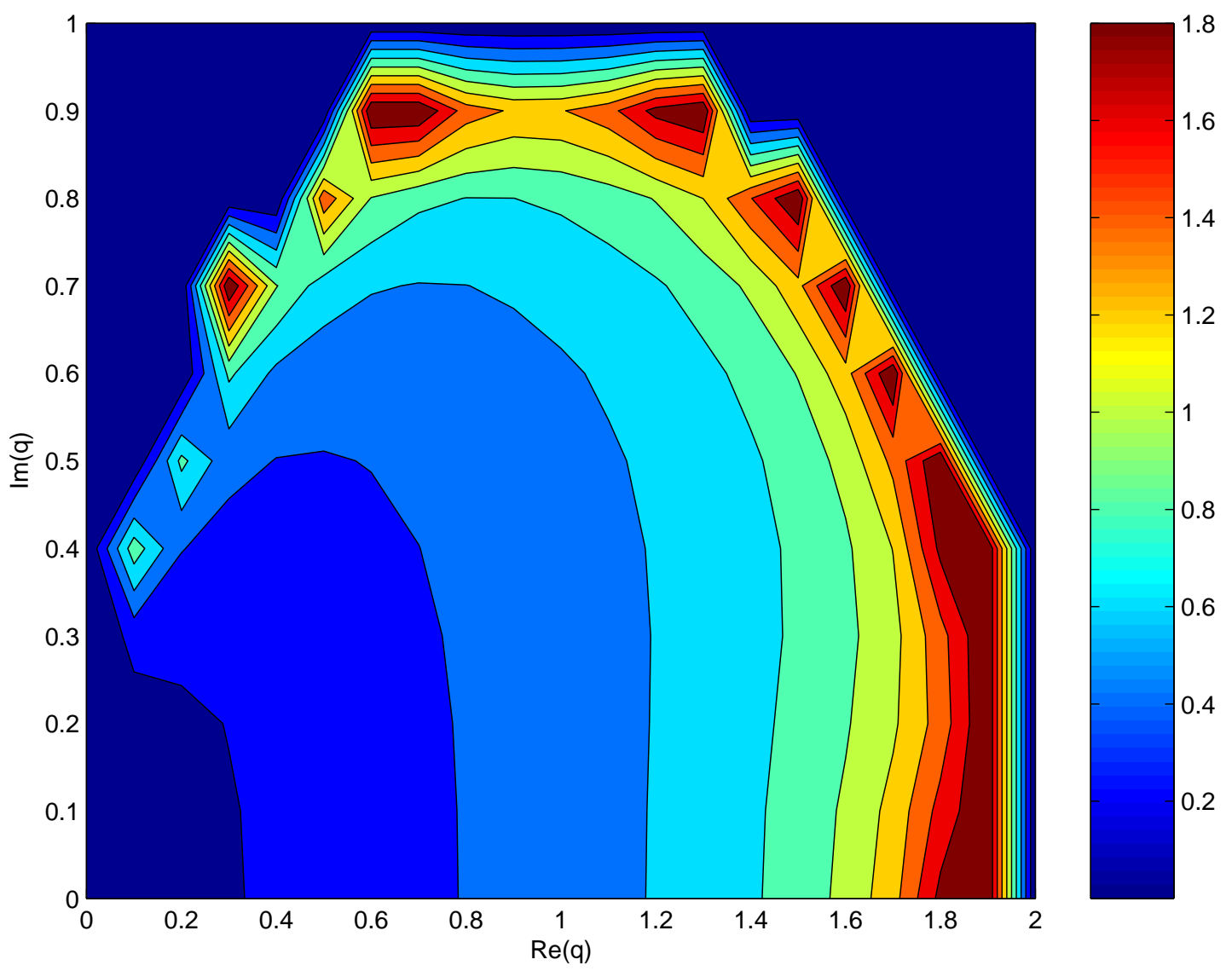

Figure 8.4: Contour Plot of the Estimates of the Hankel norm of $G(s)$ with $q \in \mathbb{C}$ such that $0 \leq \operatorname{Re}(q) \leq 2$ and $0 \leq \operatorname{Im}(q) \leq 1$. 


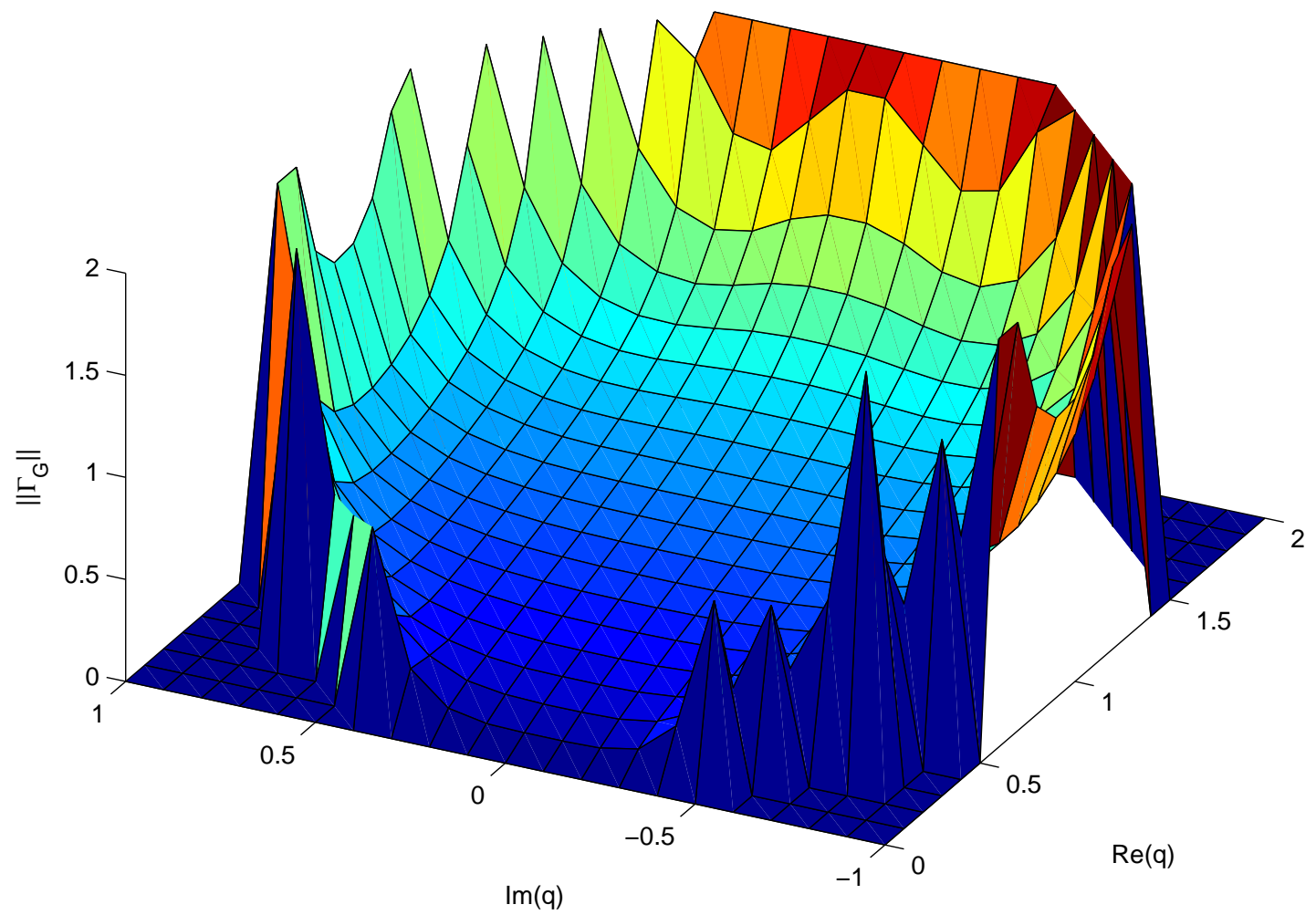

Figure 8.5: Estimates of the Hankel norm of $G(s)$ with $q \in \mathbb{C}$ such that $0 \leq \operatorname{Re}(q) \leq 2$ and $-1 \leq \operatorname{Im}(q) \leq 1$. 


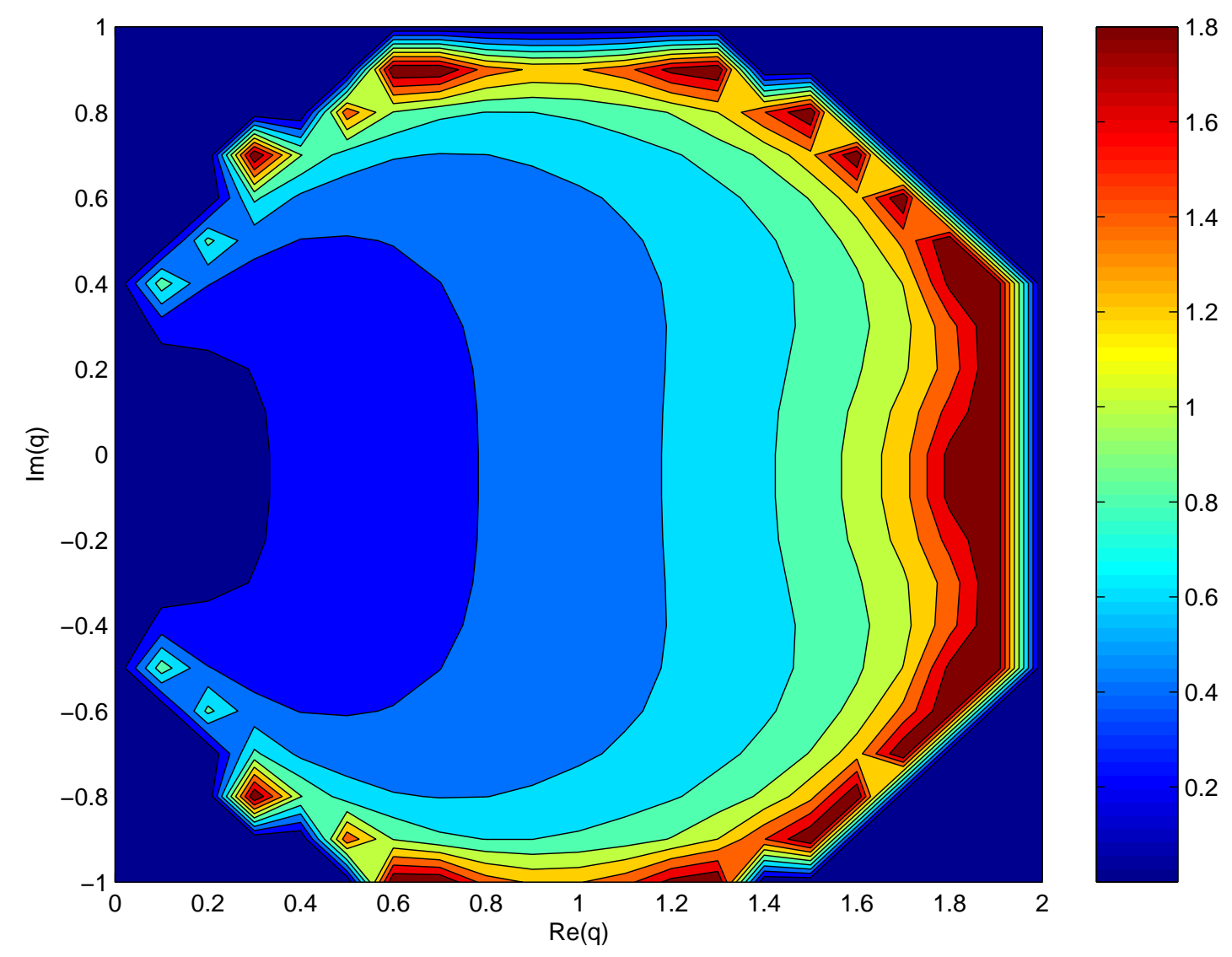

Figure 8.6: Contour Plot of the Estimates of the Hankel norm of $G(s)$ with $q \in \mathbb{C}$ such that $0 \leq \operatorname{Re}(q) \leq 2$ and $-1 \leq \operatorname{Im}(q) \leq 1$. 


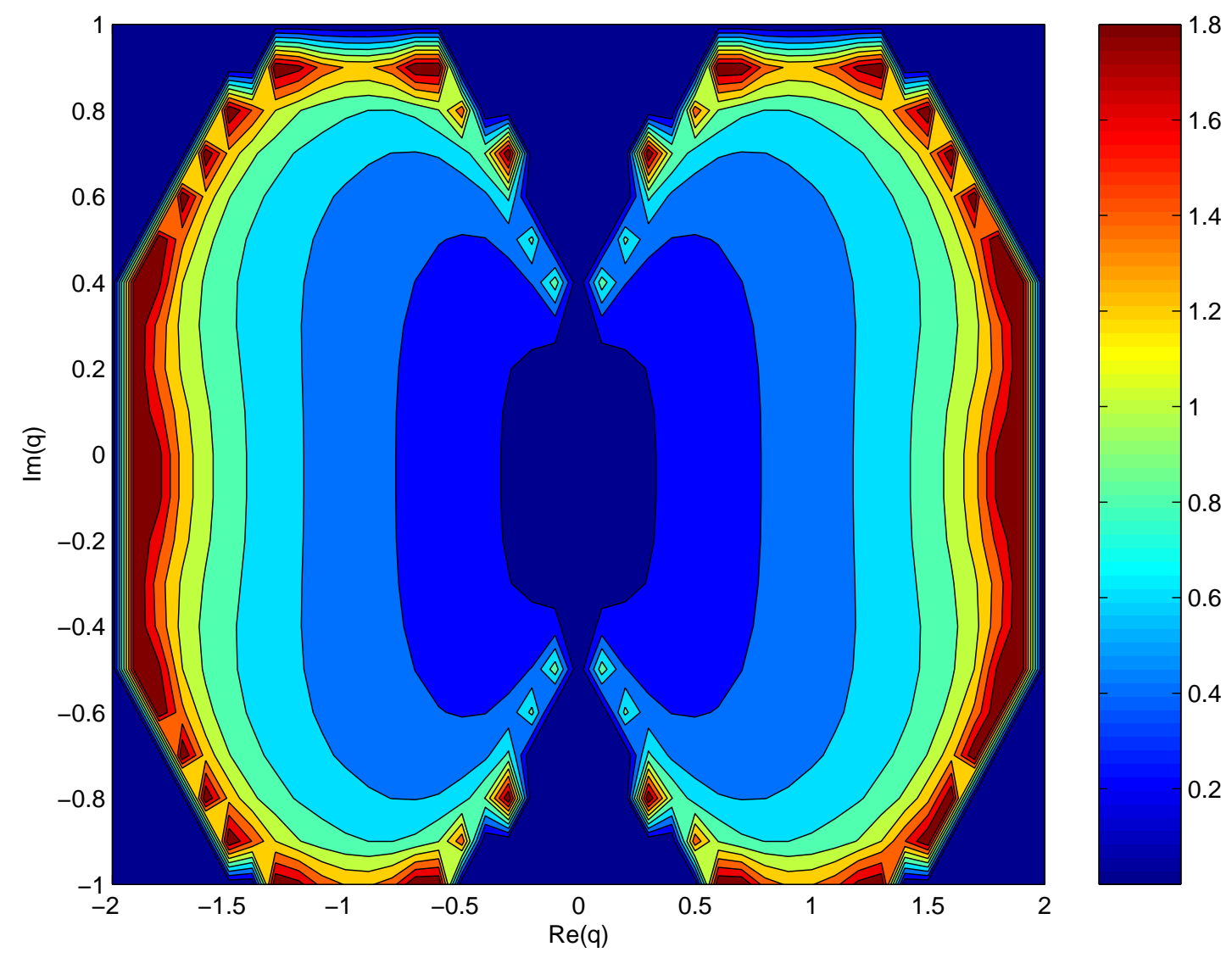

Figure 8.7: Contour Plot of the Estimates of the Hankel norm of $G(s)$ with $q \in \mathbb{C}$ such that $-2 \leq \operatorname{Re}(q) \leq 2$ and $-1 \leq \operatorname{Im}(q) \leq 1$. 


\subsection{Conclusions}

In this chapter the Hankel norm of the conjugate-order system $G(s)=\frac{\frac{1}{2}}{s^{q}+1}+\frac{\frac{1}{2}}{s^{\bar{q}}+1}$ has been estimated. The function that maps $q \in \mathbb{C}$ to the Hankel norm of $G(s)$ is symmetric about the $\operatorname{Re}(q)$ and $\operatorname{Im}(q)$ axes. The Hankel norms for $q \in(0,2)$ match the Hankel norms obtained in the previous chapter. The results given in this chapter are consistent with the analysis of conjugate-order systems given in Chapter 4. This chapter demonstrates the the Hankel singular value estimation algorithm given in Section 7.1 can be applied to systems of conjugate order as well as to systems of real order. 


\section{CHAPTER IX}

\section{CONCLUSIONS AND FUTURE WORK}

The material presented in this work represents the first steps in the determination of a spectral decomposition of the Hankel operator for fractional-order systems. An algorithm for computing the Hankel singular values of a fractional-order system has been presented. This algorithm has been applied to a class of fractional-order systems and a class of conjugate-order systems. The algorithm has also been applied to two specific fractional-order systems, the resistor-terminated and inductor-terminated semi-infinite lines.

The Hankel norm of a fractional-order system can be calculated using the method provided in Section 7.1. This calculation provides the maximum ratio of energy retrievable from the system in the future to the amount of energy delivered to the system in the past. This is an important consideration for fractional-order systems that are used as energy-storage devices. An application of this work determining the efficiency of energy storage of an ultracapacitor bank taking into account both charging and discharging losses.

The algorithm in Section 7.1 can be used to obtain information about finite-

rank approximations for fractional-order systems. This method can be applied to calculate the Hankel norm of the error between a fractional-order system and a finite- 
rank approximation, which provides a measure of the accuracy of the approximation. The $(k+1)^{\text {th }}$ Hankel singular value of a fractional-order operator obtained using this method offers the smallest achievable error between the system and a $k^{\text {th }}$-order approximation. For example, a fifth-order approximation for the resistor-terminated semi-infinite line was found using a standard approximation technique. The algorithm of Section 7.1 was applied to determine the Hankel norm of the error between the resistor-terminated semi-infinite line and the fifth-order approximation. The error was determined to be $8.6 \%$. Applying the algorithm to determine the Hankel singular values of the resistor-terminated semi-infinite line gave only $5.42 \%$ error for the best possible fifth-order approximation.

Although we have a method by which the singular values of the Hankel operator for a fractional-order system may be estimated, the solution of the Nehari problem requires the Schmidt pairs of the Hankel operator. The algorithm in Section 7.1 does provide approximations to the Schmidt pairs of the Hankel operator for a fractional-order system. However, no bound on the accuracy of those Schmidt-pair estimates is inherently obtained in the process.

A rank- $k$ approximation to the Hankel operator can be obtained, but because there is error between the actual Schmidt pairs and those obtained by the algorithm of Section 7.1, there is no guarantee on the accuracy of the rank- $k$ approximation. Further study is necessary to determine a bound on the accuracy of the Schmidt-pair estimates, and subsequently a bound on the degree by which a rank- $k$ approxima- 
tion to the Hankel operator using these estimates differs from the optimal rank- $k$ approximation.

A problem that is left for future work which builds on this dissertation is the solution of the $H_{\infty}$-control problem. The problem itself must be formulated in such a manner as to allow fractional-order systems as a plant, controller, or both plant and controller. Two possibilities immediately present themselves for systems of real and commensurate order. The first is using coprime factorizations over $\mathbb{C}\left[s^{q}\right] \cap H_{\infty}\left(\mathbb{C}^{+}\right)$, and the second using the mapping $s \mapsto w^{\frac{1}{q}}$ and coprime factorizations over $\mathbb{C}[w] \cap$ $H_{\infty}(\mathcal{W})$ where $\mathcal{W}=\left\{w \in \mathbb{C}:|\arg (w)|<q \frac{\pi}{2}\right\}$. Possible formulations are even more difficult for conjugate-order systems.

An approach analogous to the state-space approach for integer-order systems should also be considered. That approach will require the formulation of the fractional-order system as an abstract Cauchy problem of the form $\dot{x}(t)=A x(t)+$ $B u(t)$, where $A$ and $B$ are operators on an infinite-dimensional space, and $A$ probably has infinite rank. Also required is acquiring the strongly continuous semigroup generated by $A$. This approach is a standard one in the study of many infinitedimensional systems $[29,21]$. The difficulty in this approach lies in formulating the fractional-order system as an abstract Cauchy problem. 


\section{BIBLIOGRAPHY}

[1] Milton Abromowitz and Irene A. Stegun. Handbook of Mathematical Functions. Dover, 1964.

[2] B.N. Narahari Achar, Carl F. Lorenzo, and Tom T. Hartley. Initialization and the Caputo fractional derivative. Technical Report TM-2003-212482, NASA, 2003.

[3] B.N. Narahari Achar, Carl F. Lorenzo, and Tom T. Hartley. Initialization issues of the Caputo fractional derivative. In DETC05. ASME, 2005.

[4] V. M. Adamjan, D. Z. Arov, and M. G. Krein. Analytic properties of Schmidt pairs for a Hankel operator and the generalized Shur-Takagi problem. Mathematics USSR Sbornik, 15:31-73, 1971.

[5] Jay L. Adams and Tom T. Hartley. Hankel operators for fractional-order systems. Journal Européen des Systèmes Automatisés, 3(2), 2008.

[6] Jay L. Adams, Tom T. Hartley, and Lynn I. Adams. A solution to the fundamental linear complex-order differential equation. Advances in Engineering Software, March 2009.

[7] Jay L. Adams, Tom T. Hartley, and Carl F. Lorenzo. Advances in Fractional Calculus: Complex Order-Distributions Using Conjugated-Order Differintegrals, pages 347-360. Springer, New York, 2007.

[8] Jay L. Adams, Tom T. Hartley, and Robert J. Veillette. Estimation of the Hankel norm for fractional-order systems. In Proceedings of the 3rd IFAC Workshop on Fractional Differentiation and its Applications. IFAC, 2008.

[9] Huseyin Akçay. Synthesis of complete orthonormal fractional basis functions with prescribed poles. Transactions on Signal Processing, 56(10):4716-4728, October 2008.

[10] Lawrence Baggett. Functional Analysis, A Primer. Marcel-Dekker, New York, 1992. 
[11] R. L. Bagley. The thermorheologically complex material. International Journal of Engineering Science, 29(7):797-806, 1991.

[12] R. L. Bagley and R.A. Calico. Fractional order state equations for the control of viscoelastically damped structures. Journal of Guidance, Control, and Dynamics, 14(2):304-311, 1991.

[13] R. L. Bagley and P.J. Torvik. On the fractional calculus model of viscoelastic behavior. Journal of Rheology, 30(1):133-155, 1986.

[14] Vannevar Bush. Operational Circuit Analysis. J. Wiley \& Sons, New York, 1948.

[15] M Caputo. Elastica e Dissipazione. Zanichelli, Bologna, 1969.

[16] A. Chareff, H.H. Sun, Y.Y. Tsao, and B. Onaral. Fractal systems as represented by singularity function. IEEE Transactions on Automatic Control, 37:1465-1470, 1992.

[17] Wen Chen. A speculative study of fractional Laplacian modeling of turbulence. In Proceedings of the 2nd IFAC Workshop on Fractional Differentiation and its Applications. IFAC, 2006.

[18] YangQuan Chen and Kevin L. Moore. Discretization schemes for fractional-order differentiators and integrators. IEEE Transactions on Circuits and Systems I: Fundamental Theory and Applications, 49(3):363-367, 2002.

[19] John B. Conway. Functions of One Complex Variable. Springer-Verlag, New York, 2nd edition, 1978.

[20] John B. Conway. A Course in Functional Analysis. Springer-Verlag, New York, 2nd edition, 1990.

[21] Ruth F. Curtain and Hans J. Zwart. An Introduction to Infinite-Dimensional Linear Systems Theory. Springer-Verlag, New York, 1995.

[22] Vladan D. Djordjevic. Modeling of the slip boundary condition in rarefied gas microchannel flow via fractional derivative. In Proceedings of the 1 st IFAC Workshop on Fractional Differentiation and Applications, pages 153-162, July 2004.

[23] Moustafa El-Shahed. Fractional calculus model of viscoelastic fluid past a flat plate. In Proceedings of the 1st IFAC Workshop on Fractional Differentiation and Applications, pages 153-162, July 2004. 
[24] Avraham Feintuch. Robust Control Theory in Hilbert Space. Springer, 1998.

[25] M Fukunaga. Application of fractional diffusion equation to amorphous semiconductors, I. photoluminescence decays. In Proceedings of the 1st IFAC Workshop on Fractional Differentiation and Applications, pages 153-162, July 2004.

[26] Boris G. Galerkin. Stabe und flatten; reihen in gewissen gleichgewichtsproblemen elastischer stabe und flatten. Vestnik der Ingenieure, 19:897-908, 1915.

[27] W.G. Glockle and T.F. Nonnenmacher. Fractional integral operators and Fox functions in the theory of viscoelasticity. Macromolecules, 24:6426-6434, 1991.

[28] Rudolf Gorenflo and Vu Kim Tuan. Singular value decomposition of fractional integration operators in $l^{2}$ spaces with weights. Journal of Inverse and Ill-Posed Problems, 3:1-9, 1995.

[29] Jack K. Hale and Sjoerd M. Verduyn Lunel. Introduction to Functional Differential Equations. Springer, New York, 1993.

[30] Tom T. Hartley and Carl F. Lorenzo. Insights into the fractional-order initial value problem via semi-infinite systems. Technical Report TP-1998-208407, NASA, November 1998.

[31] Tom T. Hartley and Carl F. Lorenzo. A solution to the fundamental linear fractional order differential equation. Technical Report TP-1998-208693, NASA, December 1998.

[32] Tom T. Hartley and Carl F. Lorenzo. Control of initialized fractional-order systems. Technical Report TM-2002-211377, NASA, March 2002.

[33] Tom T. Hartley and Carl F. Lorenzo. Dynamics and control of initialized fractional-order systems. Nonlinear Dynamics, 29:201-233, July 2002.

[34] Tom T. Hartley, Carl F. Lorenzo, and Helen K. Qammar. Chaos in a fractional order Chua system. IEEE Transactoins on Circuits and Systems I, 42(8):485490, August 1995.

[35] Philip Hartman. On completely continuous Hankel matrices. Proceedings of the American Mathematical Society, 9, 1958.

[36] Oliver Heaviside. Electromagnetic Theory, volume 2. Chelsea Publishing Company, New York, 1922. 
[37] Nicole Heymans. Fractional order constitutive equations for viscoelasticity: sensitivity to initial state. In Proceedings of the 1st IFAC Workshop on Fractional Differentiation and Applications, pages 153-162, July 2004.

[38] Nicole Heymans and J. C. Bauwens. Fractal rheological models and fractional differential equations for viscoelastic behavior. Rheologica Acta, 33(3):210-219, May 1994.

[39] Harry Hochstadt. Integral Equations. John Wiley and Sons, New York, 1973.

[40] Cindy X. Jiang, Jay L. Adams, Joan E. Carletta, and Tom T. Hartley. Hardware implementation of fractional-order systems as infinite impulse response filters. In Proceedings of the 2nd IFAC Workshop on Fractional Differentiation and its Applications. IFAC, 2006.

[41] Cindy X. Jiang, Joan E. Carletta, and Tom T. Hartley. Advances in Fractional Calculus: Implementation of Fractional-Order Operators on Field Programmable Gate Arrays, pages 333-346. Springer, New York, 2007.

[42] Anatoly A. Kilbas, Teresa Pierantozzi, Juan J. Trujillo, and Luis Vázquez. On generalized fractional evolution-diffusion equation. In A. Le Meauté, J.A. Tenreiro, J. C. Trigeassou, and J. Sabatier, editors, Fractional Derivatives and their Applications: Mathematical tools, Geometrical and Physical aspects. Ubooks, 2005.

[43] Anatoly A. Kilbas, Hari M. Srivastava, and Juan J. Trujillo. Theory and Applications of Fractional Differential Equations. Elsevier, New York, 2006.

[44] H. Kober. On a theorem of Shur and on fractional integrals of purely imaginary order. Journal of American Mathematical Society, 50, 1941.

[45] Wilbur R. LePage. Complex Variables and the Laplace Transform for Engineers. Dover, 1961.

[46] Carl F. Lorenzo and Tom T. Hartley. Initialization, conceptualization, and application in the generalized fractional calculus. Technical Report TM-1998-208407, NASA, December 1998.

[47] Carl F. Lorenzo and Tom T. Hartley. Initialized fractional calculus. International Journal of Applied Mathematics, 3:249-265, 2000.

[48] Carl F. Lorenzo and Tom T. Hartley. Initialization in fractional-order systems. In European Control Conference, 2001. 
[49] Carl F. Lorenzo and Tom T. Hartley. Initialization of fractional differential equations: Background and theory. In DETC0\%. ASME, 2007.

[50] Carl F. Lorenzo and Tom T. Hartley. Initialization of fractional differential equations: Theory and applications. In DETC0\%. ASME, 2007.

[51] Carl F. Lorenzo and Tom T. Hartley. Application of the incomplete gamma functions to the initialization of fractional-order systems. Journal of Computational and Nonlinear Dynamics, 3(2), 2008.

[52] Carl F. Lorenzo and Tom T. Hartley. Initialization of fractional-order operators and fractional differential equations. Journal of Computational and Nonlinear Dynamics, 3(2), 2008.

[53] Carl F. Lorenzo, Tom T. Hartley, and Jay L. Adams. Inverted initialization of fractional-order systems. In Proceedings of the 3rd IFAC Workshop on Fractional Differentiation and its Applications. IFAC, 2008.

[54] E. R. Love. Fractional derivative of imaginary order. Journal of London Mathematical Society, 2:241-259, 1971.

[55] Francesco Mainardy, Gianni Pagnini, and Rudolf Gorenflo. Some aspects of fractional diffusion equations of single and distributed order. Applied Mathematics and Computation, 187(1):295-305, April 2007.

[56] Denis Matignon. Stability properties for generalized fractional differential systems. ESIAM: Proceedings, 5:145-158, 1998.

[57] R. Metzler and W. G. Glockle. Fractional model equation for anomalous diffusion. Physica A, 211:13-24, 1994.

[58] Keith S. Miller and Bertram Ross. An Introduction to the Fractional Calculus and Fractional Differential Equations. Wiley, New York, 1993.

[59] X. Moreau, O. Altet, and Alain Oustaloup. Phenomenological description of the fractional differentiation in rheology. In Proceedings of the 1st IFAC Workshop on Fractional Differentiation and Applications, pages 153-162, July 2004.

[60] Z. Nehari. On bounded bilinear forms. Annals of Mathematics, 65:153-162, 1957.

[61] Fritz Oberhettinger and Larry Badii. Tables of Laplace Transforms. SpringerVerlag, New York, 1973. 
[62] Keith B. Oldham. A new approach to the solution of electrochemical problems involving diffusion. Analytical Chemistry, 41, 1969.

[63] Keith B. Oldham. Semiintegral electroanalysis: analog implementation. Analytical Chemistry, 45(1):39-47, January 1973.

[64] Keith B. Oldham. Fractional differential equations in electrochemistry. In ECT06. Civil Comp, 2006.

[65] Keith B. Oldham, Jan C. Myland, and Jerome Spanier. An Atlas of Functions. Springer, New York, 2nd edition, 2008.

[66] Keith B. Oldham and Jerome Spanier. The Fractional Calculus, Integrations and Differentiations of Arbitrary Order. Academic Press, New York, 1974.

[67] Alan V. Oppenheim, Alan S. Willsky, and S. Hamid Nawab. Signals and Systems. Prentice-Hall, Upper Saddle River, NJ, 2nd edition, 1997.

[68] Manuel D. Ortigueira. On the initial conditions in continuous time fractional linear systems. Signal Processing, 83:2301-2309, 2003.

[69] Alain Oustaloup, F. Levron, B. Mathieu, and F. M. Nanot. Frequency-band complex noninteger differentiator: Characterization and synthesis. IEEE Transactions on Circuits and Systems I, 47:25-39, 2000.

[70] Alain Oustaloup and Jocelyn Sabatier. Study of the relaxation of water on a porous dyke: a non-integer approach. Annales Geophysicae, 13, 1995.

[71] Alain Oustaloup, Jocelyn Sabatier, and Patrick Lanusse. From fractal robustness to the CRONE control. Fractional Calculus and Applied Analysis, 2:1-30, 1999.

[72] Bruce P. Palka. An Introduction to Complex Function Theory. Springer, New York, 1991.

[73] Jonathan R. Partington. An Introduction to Hankel Operators. Cambridge University Press, Cambridge, 1988.

[74] Jonathan R. Partington. Linear Operators and Linear Systems: An Analytical Approach to Control Theory. Cambridge University Press, Cambridge, 2004.

[75] Vladimir V. Peller. Hankel Operators and Their Applications. Springer, New York, 2003. 
[76] Allen C. Pipkin. A Course on Integral Equations. Springer-Verlag, 1991.

[77] Igor Podlubny. Fractional Differential Equations, An Introduction to Fractional Derivatives, Fractional Differential Equations, Some Methods of Their Solution and Some of Their Applications. Academic Press, New York, 1999.

[78] David Porter and David S. G. Stirling. Integral Equations, a Practical Treatment from Spectral Theory to Applications. Cambridge University Press, Cambridge, 1990.

[79] Lynnette E. S. Ramirez and Carlos F. M. Coimbra. A variable order constitutive relation for viscoelasticity. Annalen der Physik, 16:543-552, 2007.

[80] John W. Rayleigh. In finding the correction for the open end of an organ-pipe. Philosophical Transactions of the Royal Society, 161, 1870.

[81] Walther Ritz. Uber eine neue methode zur losung gewizzer variationsprobleme der mathematischen physik. Journal fur die Reine und Angewandte Mathematik, 135:1-61, 1908.

[82] Bryan P. Rynne and Martin A. Youngson. Linear Functional Analysis. SpringerVerlag, London, 2nd edition, 2008.

[83] S. G. Samko, A. A. Kilbas, and O. I. Marichev. Fractional Integrals and Derivatives: Theory and Applications. Gordon and Breach Science Publishers, Philadelphia, PA, 1993.

[84] Abul Hasan Siddiqi. Applied Functional Analysis: Numerical Methods, Wavelet Methods, and Image Processing. Marcel Dekker, New York, 2004.

[85] W. Smit and H. deVries. Rheological models containing fractional derivatives. Rheologica Acta, 9:525-534, 1970.

[86] Chia-Chi Tsui. Robust Control System Design: Advanced State Space Techniques. Marcel Dekker, Inc, New York, 2nd edition, 2004.

[87] Nicholas Young. An Introduction to Hilbert Space. Cambridge University Press, 1988. 
APPENDICES 


\title{
APPENDIX A
}

\section{LIST OF SYMBOLS}

\author{
$\mathcal{B}(\mathcal{X}) \quad \mathcal{B}(\mathcal{X}, \mathcal{X})$ \\ $\mathcal{B}(\mathcal{X}, \mathcal{Y}) \quad$ For Banach spaces $\mathcal{X}$ and $\mathcal{Y}$, the set of bounded linear \\ operators from $\mathcal{X}$ to $\mathcal{Y}$ \\ $\mathcal{B}_{0}(\mathcal{X}) \quad \mathcal{B}_{0}(\mathcal{X}, \mathcal{X})$ \\ $\mathcal{B}_{0}(\mathcal{X}, \mathcal{Y})$ For Banach spaces $\mathcal{X}$ and $\mathcal{Y}$, the set of compact operators \\ from $\mathcal{X}$ to $\mathcal{Y}$ \\ $\mathcal{B}_{00}(\mathcal{X}) \quad \mathcal{B}_{00}(\mathcal{X}, \mathcal{X})$ \\ $\mathcal{B}_{00}(\mathcal{X}, \mathcal{Y})$ For Banach spaces $\mathcal{X}$ and $\mathcal{Y}$, the set of finite-rank oper- \\ ators from $\mathcal{X}$ to $\mathcal{Y}$ \\ $\mathbb{C} \quad$ The complex numbers \\ $\mathbb{C}^{+} \quad$ The set $\{z \in \mathbb{C}: \operatorname{Re}(z)>0\}$ \\ $\mathbb{C}^{-} \quad$ The set $\{z \in \mathbb{C}: \operatorname{Re}(z)<0\}$ \\ $C(\mathbb{T}) \quad$ The set of continuous functions on $\mathbb{T}$ \\ $C_{0}(i \mathbb{R}) \quad$ The set of continuous functions on $i \mathbb{R}$ with limit zero as \\ $\omega \rightarrow \infty$ \\ $\mathbb{D} \quad$ The unit disc; the set $\{z \in \mathbb{C}:|z|<1\}$ \\ $\mathcal{E}_{\alpha}(z) \quad$ The Mittag-Leffler function \\ $\mathcal{E}_{\alpha, \beta}(z) \quad$ The generalized Mittag-Leffler function
}


$E_{t}(q) \quad$ The function of Miller and Ross

$\mathbb{F} \quad$ A field, typically either $\mathbb{C}$ or $\mathbb{R}$

$F_{q}(a, t)$ The $F$-Function, the Green's function for ${ }_{0} d_{t}^{q} x(t)-$ $a x(t)=u(t)$

$\Gamma \quad$ A Hankel operator on $\ell_{2}(\mathbb{N})$

$\Gamma_{G} \quad$ A Hankel operator on $H_{2}\left(\mathbb{C}^{+}\right)$

$\Gamma_{g} \quad$ A Hankel operator on $H_{2}(\mathbb{D})$

$\Gamma_{\mathcal{G}} \quad$ A Hankel operator on $L_{2}\left(\mathbb{R}_{+}\right)$

$\Gamma(z) \quad$ The (complete) gamma function

$\Gamma(a, z) \quad$ The upper incomplete gamma function

$\gamma(a, z) \quad$ The lower incomplete gamma function

$\gamma^{*}(a, z) \quad$ The entire lower incomplete gamma function

$H_{2}(\mathbb{D}) \quad$ The set $\left\{g \in L_{2}(\mathbb{T}): g(z)\right.$ is analytic in $\left.\mathbb{D}\right\}$

$H_{2}\left(\mathbb{C}^{+}\right)$The set $\left\{F \in L_{2}(i \mathbb{R}): F(s)\right.$ is analytic in $\left.\operatorname{Re}(s)>0\right\}$

$H_{2}\left(\mathbb{C}^{-}\right)$The set $\left\{F \in L_{2}(i \mathbb{R}): F(s)\right.$ is analytic in $\left.\operatorname{Re}(s)<0\right\}$

$H_{\infty}\left(\mathbb{C}^{+}\right)$The set $\left\{G \in L_{\infty}(i \mathbb{R}): G(s)\right.$ is analytic in $\left.\operatorname{Re}(s)>0\right\}$

$\operatorname{Im}(z) \quad$ The imaginary part of $z$

$\mathcal{L}\{x(t)\}$ The bilateral Laplace transform of $x(t)$

$L_{2}(\mathbb{R}) \quad$ The set $\left\{f: \int_{-\infty}^{\infty}|f(t)|^{2} d t<\infty\right\}$ 
$L_{2}\left(\mathbb{R}_{+}\right)$The set $\left\{f \in L_{2}(\mathbb{R}): f(t)=0\right.$ for $\left.t<0\right\}$

$L_{2}\left(\mathbb{R}_{-}\right)$The set $\left\{f \in L_{2}(\mathbb{R}): f(t)=0\right.$ for $\left.t \geq 0\right\}$

$L_{2}(i \mathbb{R}) \quad$ The set $\left\{\hat{f}: \int_{-\infty}^{\infty}|\hat{f}(j \omega)|^{2} d \omega<\infty\right\}$

$L_{2}(\mathbb{T}) \quad$ The set $\left\{g: \int_{-\infty}^{\infty}\left|g\left(e^{j \theta}\right)\right|^{2} d \theta<\infty\right\}$

$L_{\infty}(i \mathbb{R})$ The set $\{G:|G(j \omega)|$ has a uniform bound on $j \mathbb{R}\}$

$\lambda_{n}(K) \quad$ For $K \in \mathcal{B}(\mathcal{H})$, the $n^{\text {th }}$ eigenvalue of $K$

$\mathbb{N} \quad$ The natural numbers; the set $\{0,1,2,3, \cdots\}$

$\Pi_{+} \quad$ The orthogonal projection operator from $L_{2}(i \mathbb{R})$ onto $H_{2}\left(\mathbb{C}^{+}\right)$or from $L_{2}(\mathbb{T})$ onto $H_{2}(\mathbb{D})$

$\Pi_{-} \quad$ The orthogonal projection operator from $L_{2}(i \mathbb{R})$ onto $H_{2}\left(\mathbb{C}^{-}\right)$or from $L_{2}(\mathbb{T})$ onto $H_{2}(\mathbb{D})^{\perp}$

$\Pi_{E} \quad$ The projection operator from $\mathcal{H}$ onto $E \subset \mathcal{H}$

$\mathbb{R} \quad$ The real numbers

$\mathbb{R}^{N} \quad$ The set of all $N$-tuples with real entries

$\mathbb{R}_{+} \quad$ The positive real numbers, the set $\{x \in \mathbb{R}: x>0\}$

$\mathbb{R}_{-} \quad$ The negative real numbers, the set $\{x \in \mathbb{R}: x<0\}$

$\mathbb{R}[s] \quad$ The ring of polynomials in $s$ with coefficients in $\mathbb{R}$

$\mathbb{R}(s) \quad$ The field of ratios of $\mathbb{R}[s]$; the set

$\left\{\frac{B(s)}{A(s)}: A(s), B(s) \in \mathbb{R}[s]\right\}$

$R_{q, \nu}(a, t)$ The $R$-Function, the Green's function for ${ }_{0} d_{t}^{q} x(t)-$ $a x(t)={ }_{0} d_{t}^{\nu} u(t)$ 
range $(K)$ For $K: A \rightarrow B$, the range of $K$, the set $\{b \in B: b=K a$ for some $a \in A\}$

$\operatorname{rank}(K) \quad$ For an operator $K$, the dimension of range $(K)$

$\operatorname{Re}(z) \quad$ The real part of $z$

$R H_{\infty}\left(\mathbb{C}^{+}\right)$The set $H_{\infty}\left(\mathbb{C}^{+}\right) \cap \mathbb{R}(s)$

$\mathcal{S} \quad$ The Schwartz space of rapidly decreasing functions on $\mathbb{R}$

$\operatorname{sgn}(r) \quad$ The signum of $r$, for $r \in \mathbb{R}, \operatorname{sgn}(r)=\frac{r}{|r|}$

$\sigma_{n}(K) \quad$ For $K \in \mathcal{B}(\mathcal{H})$, the $n^{\text {th }}$ singular value of $K$

$\mathbb{T} \quad$ The unit circle; the set $\{z \in \mathbb{C}:|z|=1\}$

$\mathcal{U}_{s}(t) \quad$ The unit step function, $\mathcal{U}_{s}(t)= \begin{cases}1, & t \in[0, \infty) \\ 0, & t \in(-\infty, 0)\end{cases}$

$\mathbb{Z} \quad$ The integers; the set $\{\cdots-2,-1,0,1,2, \cdots\}$ 


\section{APPENDIX B}

\section{MATHEMATICA CODE}

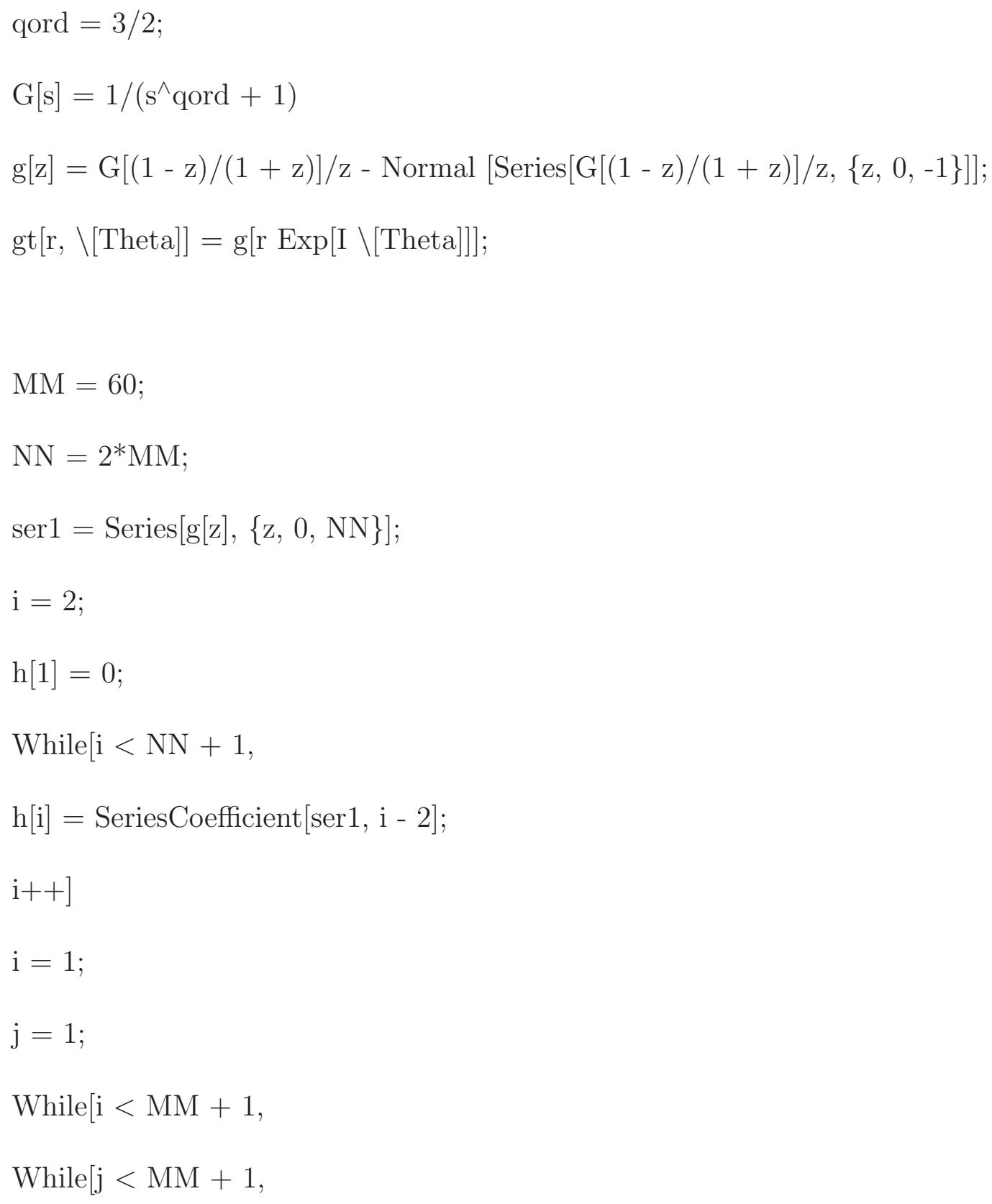




$$
\begin{aligned}
& \mathrm{A}[\mathrm{i}, \mathrm{j}]=\mathrm{h}[\mathrm{i}+\mathrm{j}] ; \\
& \mathrm{j}++] ; \\
& \mathrm{j}=1 ; \\
& \mathrm{i}++] \\
& \text { Amat = Table[A[i, j] // N, }\{\mathrm{i}, 1, \mathrm{MM}\},\{\mathrm{j}, 1, \mathrm{MM}\}] ; \\
& \text { Ev = Eigenvalues[Amat]; }
\end{aligned}
$$

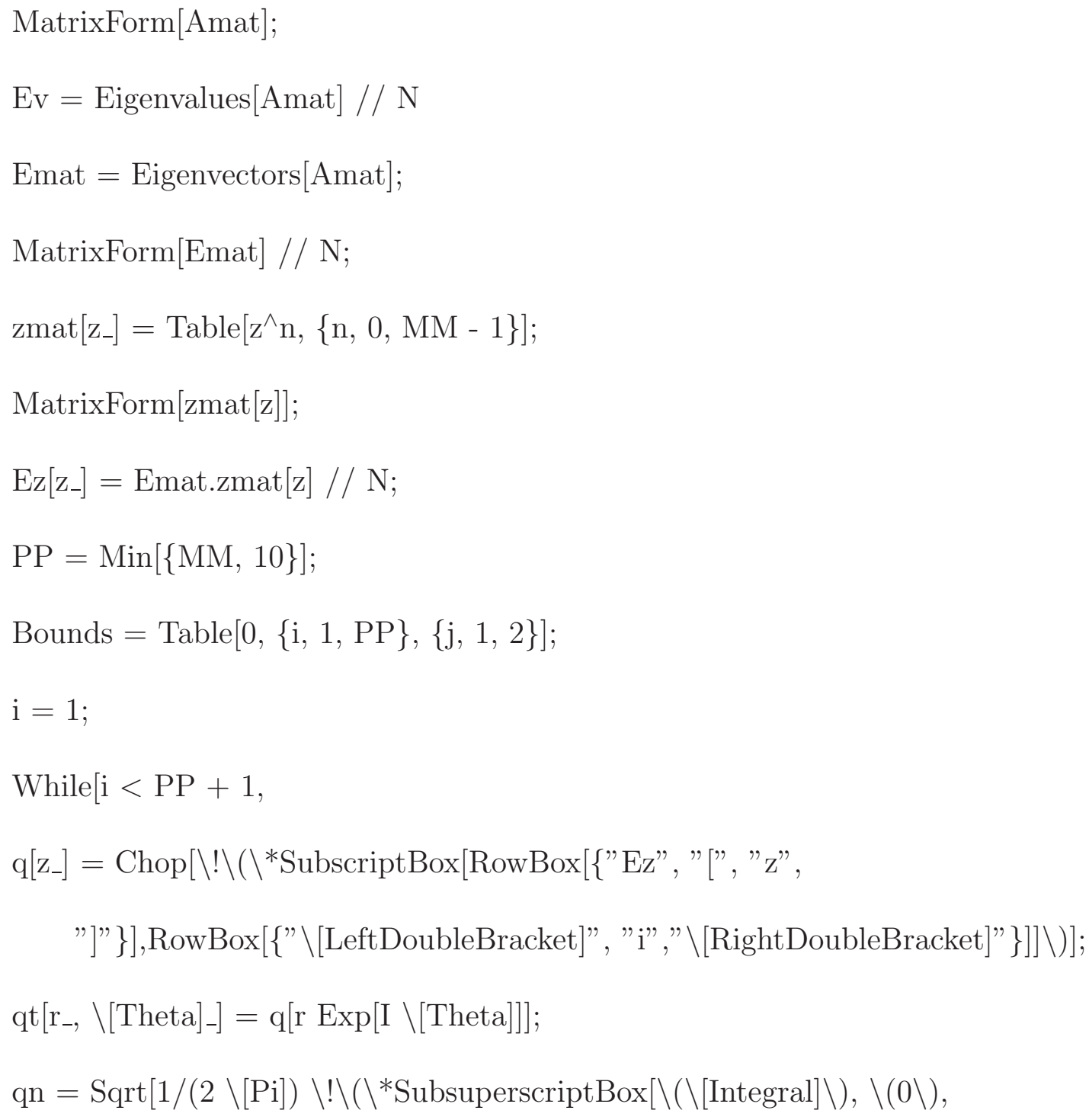


$\backslash(2 \backslash \backslash[\mathrm{Pi}] \backslash)] \backslash(\mathrm{qt}[1, \backslash[$ Theta $]] \backslash \mathrm{qt}[1, \backslash(-\backslash[$ Theta $] \backslash)] \backslash[$ DifferentialD $] \backslash[$ Theta $] \backslash) \backslash)]$;

$\mathrm{p}\left[\mathrm{z}_{-}\right]=\mathrm{q}[\mathrm{z}] / \mathrm{qn} ;$

$\mathrm{pt}\left[\mathrm{r}_{-}, \backslash[\text { Theta }]_{-}\right]=\mathrm{p}[\mathrm{r} \operatorname{Exp}[\mathrm{I} \backslash[$ Theta $]]] ;$

$\mathrm{Kpb}\left[\mathrm{z}_{-}\right]=\mathrm{g}[\mathrm{z}] \mathrm{p}\left[\mathrm{z}^{\wedge}-1\right]$

$\mathrm{Kp}[\mathrm{z}]]=\operatorname{Kpb}[\mathrm{z}]-\operatorname{Normal}[\operatorname{Series}[\operatorname{Kpb}[\mathrm{z}],\{\mathrm{z}, 0,-1\}]] ;$

$\operatorname{Kpt}\left[\mathrm{r}_{-}, \backslash[\text { Theta }]_{-}\right]=\operatorname{Kp}[\mathrm{r} \operatorname{Exp}[\mathrm{I} \backslash[$ Theta $]]] ;$

Kppn $=\backslash ! \backslash\left({ }^{*}\right.$ SubscriptBox $[" E v "$,

RowBox[\{"\[LeftDoubleBracket]", "i", "\[RightDoubleBracket]"\}]]\);

$\operatorname{mer}\left[\mathrm{z}_{-}\right]=\mathrm{Kp}[\mathrm{z}]-\mathrm{Kppn} \mathrm{p}[\mathrm{z}]$

$\operatorname{mert}\left[\mathrm{r}_{-}, \backslash[\text { Theta }]_{-}\right]=\operatorname{mer}[\mathrm{r} \operatorname{Exp}[\mathrm{I} \backslash[$ Theta $]]]$;

mern $=\backslash[\operatorname{Sqrt}]\left(2 /(2 \backslash[\mathrm{Pi}])\right.$ NIntegrate $\left[\operatorname{Abs}[\operatorname{mert}[1, \backslash[\text { Theta }]]]^{\wedge} 2,\{\backslash[\right.$ Theta $], 0, \backslash[\mathrm{Pi}]$

\}]);

$\mathrm{JK}=\operatorname{Abs}[\mathrm{Kppn}]$

$\mathrm{EK}=$ mern

$\backslash ! \backslash\left(*^{*}\right.$ SubscriptBox["Bounds", RowBox[[" \[LeftDoubleBracket]", "i", "\[RightDoubleBracket]" $\}]] \backslash)=\{\mathrm{JK}, \mathrm{JK}+\mathrm{EK}\} / /$ Chop;

$\mathrm{i}++]$

MatrixForm[Bounds]

Boundsmat $=$ Flatten $[\{$ Bounds, $\{\{$ qord, 1$\},\{0, \mathrm{MM}\}\}\}, 1]$;

Export[" c:\data.dat", Boundsmat, "Table"];

Export["c:\data.xls", Bounds, "XLS"]; 